\title{
HAEMANGIOMA: A STUDY OF THE BIOLOGY
}

\author{
By \\ Tinte Itinteang
}

\begin{abstract}
A thesis
Submitted to the Victoria University of Wellington

In fulfillment of the requirements for the degree of

Doctor of Philosophy

In Cell and Molecular Biology
\end{abstract}

Victoria University of Wellington

2012 


\section{Table of Contents}

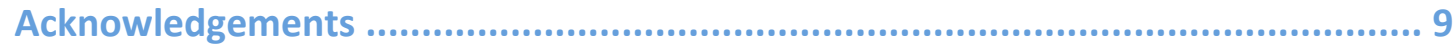

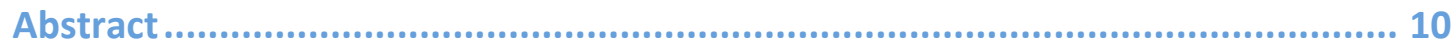

Chapter 1: Introduction..................................................................... 14

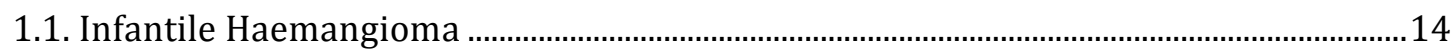

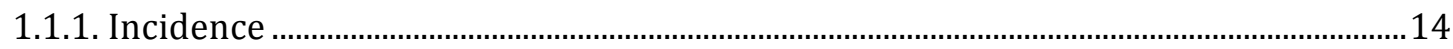

1.1.2. Clinical Features of Infantile Haemangioma ......................................................................14

1.1.3. Subtypes of Infantile Haemangioma ………………..........................................................16

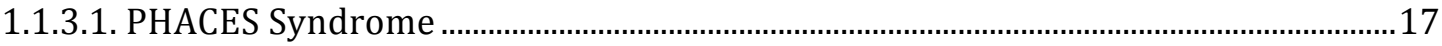

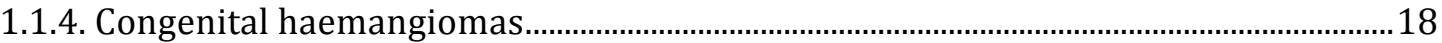

1.1.5. Complications and Indications for Treatment of Infantile Haemangioma .................19

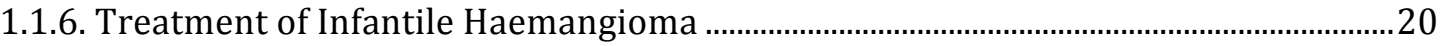

1.2. Cellular Elements of Infantile Haemangioma...................................................................2

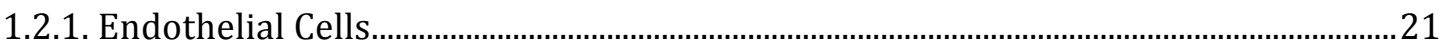

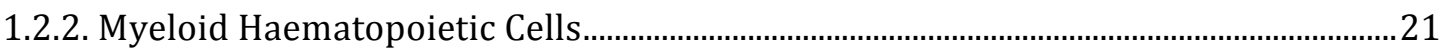

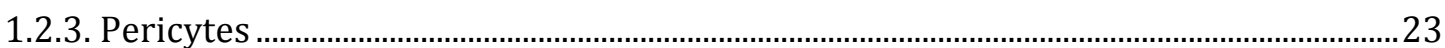

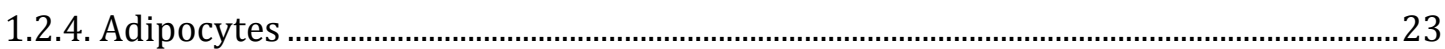

1.3. Stem Cell Theories of the Origin of Infantile Haemangioma ...............................................23

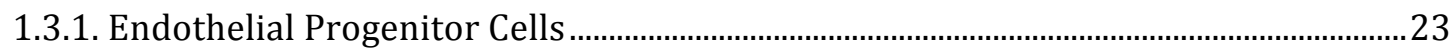

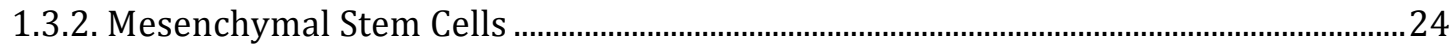

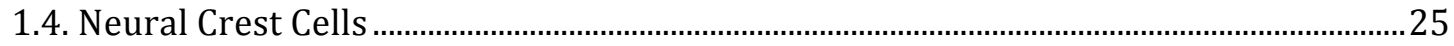

1.4.1. Segmental Migration and Its Role in Embryogenesis .....................................................25

1.4.2. Characterisation and Identification ................................................................................26

1.4.3. Embryonic Stem Cell Origin of Neural Crest Cells ........................................................2

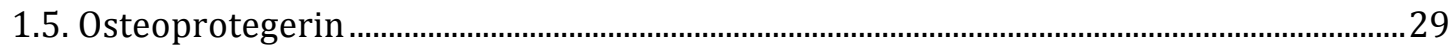


1.5.1. Osteoprotegerin and Receptor for Nuclear Factor $\kappa \mathrm{B}$ Ligand

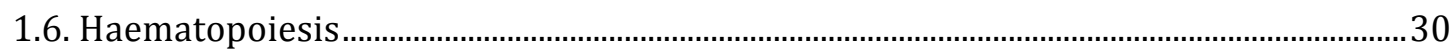

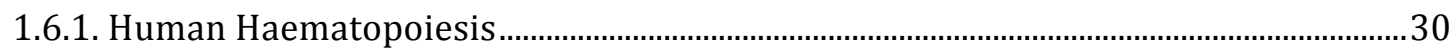

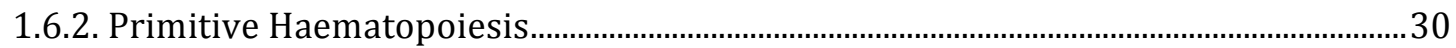

1.6.3. Definitive Haematopoiesis............................................................................................... 31

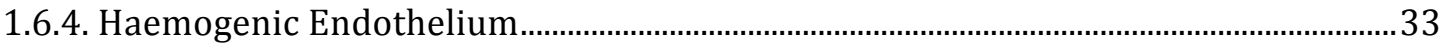

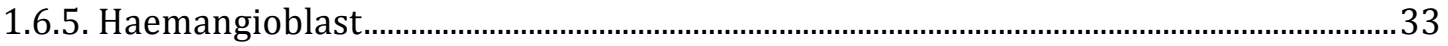

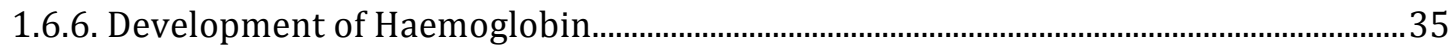

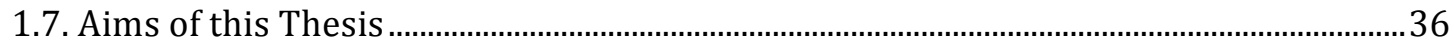

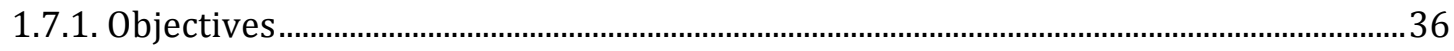

Chapter 2: General Methods .......................................................... 37

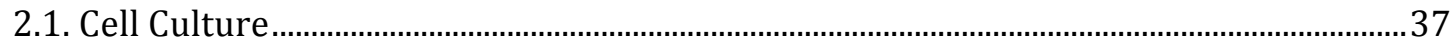

2.1.1. Infantile Haemangioma Tissue Specimens.........................................................................

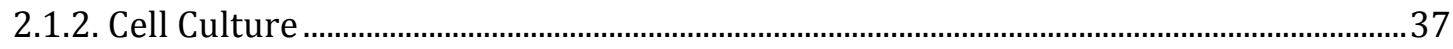

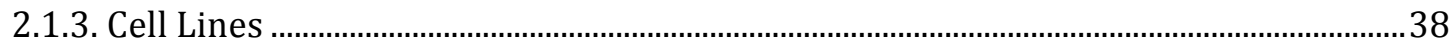

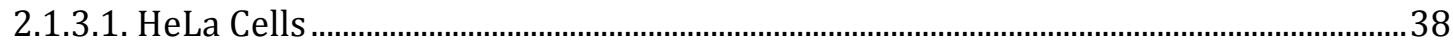

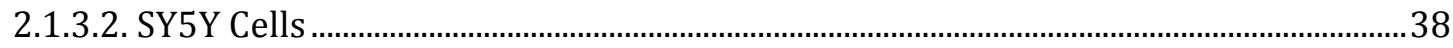

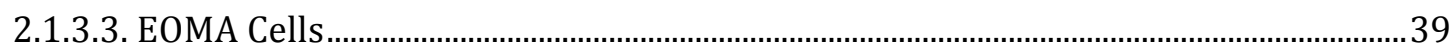

2.1.4. Cell Culture Techniques ............................................................................................... 39

2.2. Infantile Haemangioma Tissue Culture ………................................................................... 40

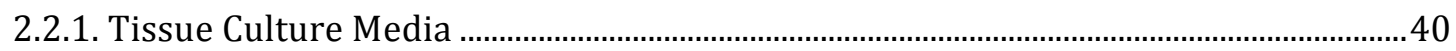

2.2.2. Infantile Haemangioma Tissue Specimen Preparation......................................................40

2.2.3. Infantile Haemangioma Explant In Vitro Culture................................................................ 41

2.2.4. Isolation of Infantile Haemangioma Explant Derived Cells..............................................4

2.2.4. Angiotensin II and Blast Proliferation ……...................................................................... 42

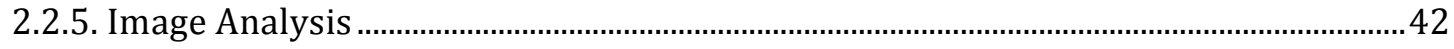

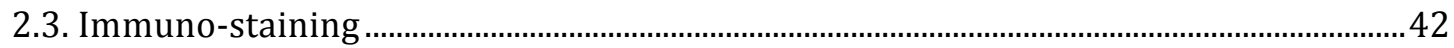


2.3.1. Immunohistochemistry Slide Preparation

2.3.2. Paraffin Tissue Sections

2.3.3. Immunocytochemistry Slide Preparation.......................................................................... 43

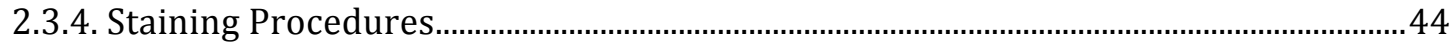

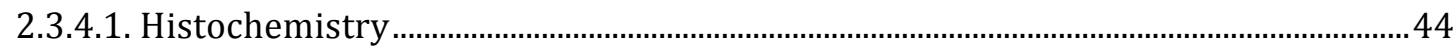

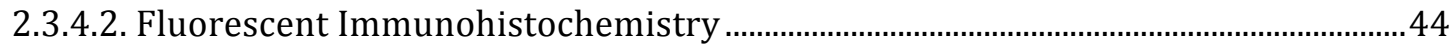

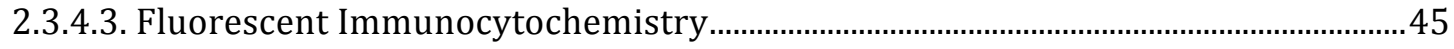

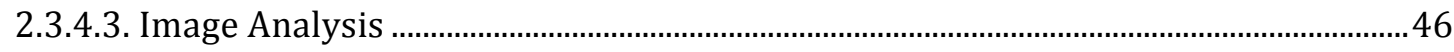

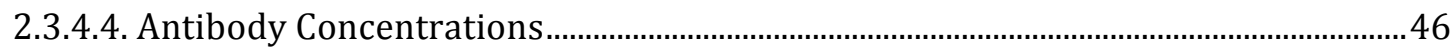

Chapter 3: Primitive Mesodermal Cells with A Neural Crest Stem Cell Phenotype In Proliferating Infantile Haemangioma ....................................................... 48

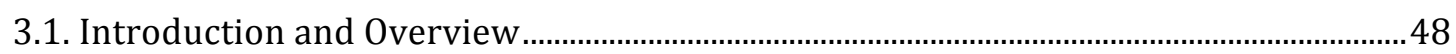

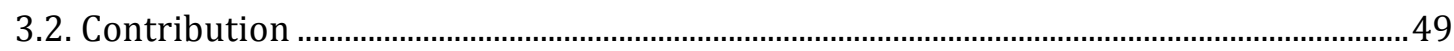

3.3. Manuscript: Primitive Mesodermal Cells With A Neural Crest Stem Cell Phenotype

Predominate Proliferating Infantile Haemangioma . 49

\section{Chapter 4: Expression of Haemogenic Endothelium Markers In Infantile}

Haemangioma ............................................................................. 56

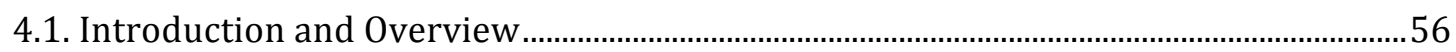

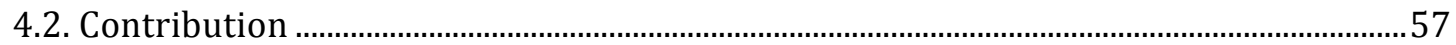

4.3. Manuscript: Haemogenic Endothelium In Infantile Haemangioma ..................................57

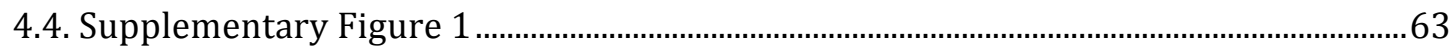

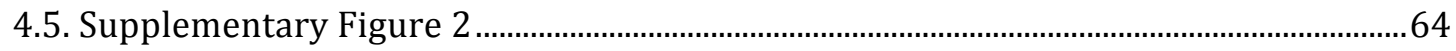

Chapter 5: Primitive Erythropoiesis in Infantile Haemangioma........................ 65

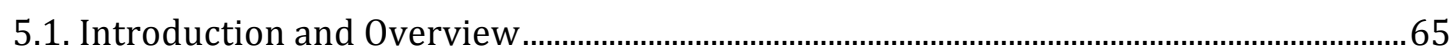

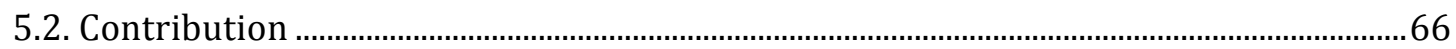

5.3. Manuscript: Primitive Erythropoiesis In Infantile Haemangioma......................................66

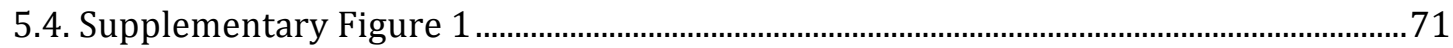


CHAPTER 6: Mesenchymal Stem Cells in Infantile Haemangioma

6.2. Contribution

6.3. Manuscript: Mesenchymal Stem Cells In Infantile Haemangioma

Chapter 7: Osteoprotegerin and Receptor for Nuclear Factor $к \beta$ in Infantile Haemangioma ............................................................................... 80

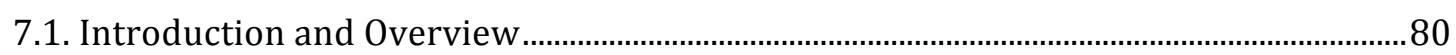

7.2. Contribution 80

7.3. Manuscript: Infantile Haemangioma Expresses Tumour Necrosis Factor-Related Apoptosis-Inducing Ligand (TRAIL), TRAIL Receptors, Osteoprotegerin And Receptor Activator For Nuclear Factor K $\beta$ Ligand (RANKL)

Chapter 8: Renin-Angiotensin System and Infantile Haemangioma .

8.1. Introduction and Overview

8.2. Contribution

8.3. Manuscript: Expression Of Components Of The Renin- Angiotensin System In Proliferating Infantile Haemangioma May Account For Propranolol May Account For Propranolol-Induced Accelerated Involution

8.4. Controls 103

Chapter 9: Embryonic Stem Cell Markers in Infantile Haemangioma 104

9.1. Introduction and Overview 104

9.2. Contribution 105

9.3. Manuscript: Expression Of Embryonic Stem Cell Markers In Infantile Haemangioma

Chapter 10: Placental Chorionic Villous Mesenchymal Core Cells and Infantile Haemangioma

10.1. Introduction and Overview 124

10.2. Contribution 125 
10.3. Manuscript: A Placental Chorionic Villous Mesenchymal Core Cellular Origin For Infantile Haemangioma.

Chapter 11: Treatment of Infantile Haemangioma

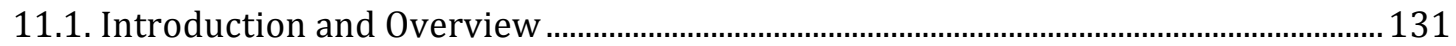

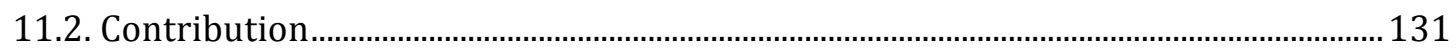

11.3. Manuscript: Low-Dose Propranolol For Infantile Haemangioma ................................ 132

11.4. Manuscript: Low-Dose Propranolol For Multiple Infantile Hepatic And Cutaneous

Hemangiomas With Deranged Liver Function 141

11.5. Manuscript: Pharmacologic Therapies For Infantile Hemangioma: Is There A

Rational Basis?

11.6. Manuscript: Treatment Of Infantile Hemangioma With Captopril 157

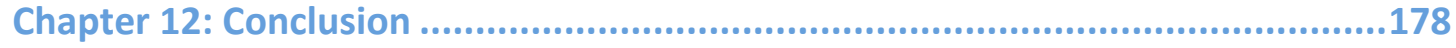

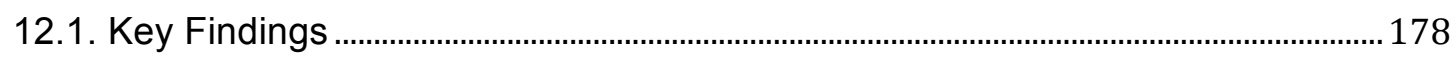

12.1.1. Renin-Angiotensin System in Infantile Haemangioma ....................................... 178

12.1.2. Osteoprotegerin in Haemangioma …………...................................................... 179

12.1.3. Haemogenic Endothelium and Primitive Erythropoiesis in Infantile

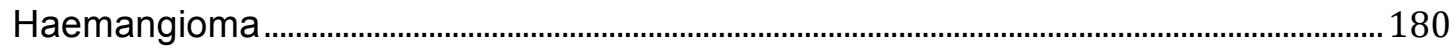

12.1.4. Primitive Mesoderm with a Neural Crest Stem cell phenotype in

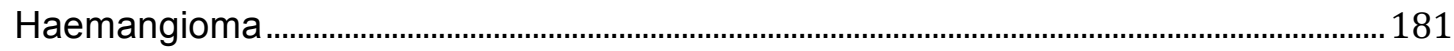

12.1.5. Mesenchymal Stem Cells in Haemangioma ......................................................... 181

12.1.6. Embryonic Stem Cell expression in Infantile Haemangioma ............................. 182

12.1.7. Placental Chorionic Villous Mesenchymal core cellular origin of Infantile

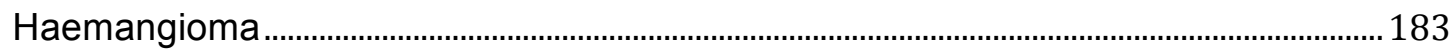

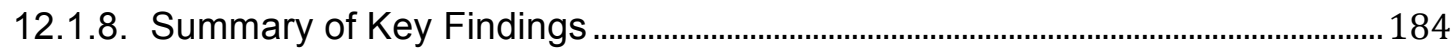

12.2. Future Directions in Haemangioma Research .............................................................. 186

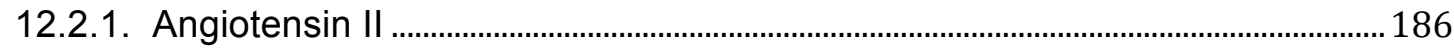

12.2.2. Stem Cell Biology of Infantile Haemangioma....................................................... 188 


\section{List of Figures}

\section{Chapter 1: Introduction}

Figure 1.1: Serial images of a child with haemangioma

Figure 1.2: Haematoxylin and eosin staining of a haemangioma ..........................................16

Figure 1.3: Loaclised focal haemangiomas on the forehead ..................................................17

Figure 1.4: Segmental haemangiomas .................................................................................18

Figure 1.5: A child with PHACES syndrome ………….............................................................

Figure 1.6: Mast cells in proliferating haemangioma

Figure 1.7: Neural crest cell lineage differentiation capacity ....................................................27

Figure 1.8: Lineage characterisation and differentiation of ESC- derived neural crest stem cells

Figure 1.9: The chronological appearance of haematopoietic sites in the human

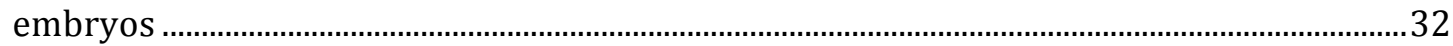

Figure 1.10: IHC staining of a human bone marrow logette .......................................................33

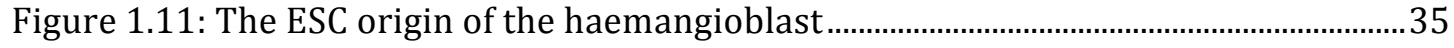

Figure 1.12: Schematic timeline for human globin genes ...........................................................36 


\section{Chapter 12: Conclusion}

Figure 12.1: Proposed model for the cellular lineages of haemangioma and the role of the renin-angiotensin system (RAS) \& osteoprotegerin 193

Figure 12.2: IHC of Cyclo-Oxygenase-2 and cathepsin B in profilerating haemangioma 194

Figure 12.3: RAS \& non-RAS pathways leading to angiotensin II formation 195

Figure 12.4: Develepment of an in vivo mouse haemangioma model. 196

Figure 12.5: Future clinical applications of tissue engineering... 197

Figure 12.6: The formation of vasculr tubes from endotheloioma tumour cells requires the presence of haemangioma stem cells. 198 


\section{List of Tables}

Chapter 1: Introduction

Table 1.1: Features of haemangioma with the highest risk of morbidity 


\section{Acknowledgements}

I would like to firstly thank my supervisors Prof Swee Tan and Dr Darren Day, for providing invaluable guidance, support, encouragement, critical discussion and for believing in me throughout the course of this project.

I would also like to thank the support of Dr Helen Brasch, Elizabeth Jones, and the staff members of the Hutt hospital histology laboratory, whose efforts in providing me with the prepared specimens were essential for the completion of this project.

To Prof John Miller, Dr Ryan Steele and Dr Arun Kanarkar, many thanks for the great discussions and laboratory tips throughout the years.

A word of appreciation to my parents, Itinteang \& Nancy, my mother-in-law, Aren, my uncle and aunt, Toto \& Miita, my sister, Angela, and to the rest of my friends and family whose support has not gone unnoticed.

Lastly a very big appreciation goes to my wife, Tetaake, and my sons, Andrew, Aaron and Tong, for supporting me, believing in me, reminding me of life's most significant elements and for their limitless love throughout the course of this work.

I would also like to acknowledge the sponsors who funded this work including the Gillies McIndoe Foundation, the Wellington Regional Plastic Surgery Unit Research and Education Trust, Victoria University of Wellington Research Trust, the Wellington Surgical Research Trust, and the Royal Australasian College of Surgeons' Foundation for Surgery Scholarship. 


\section{Abstract}

Infantile haemangioma $(\mathrm{IH})$, considered a primary tumour of the microvasculature, is the most common tumour of infancy affecting about $10 \%$ of Caucasian infants. IH predominantly affects white, female and premature infants. IH typically undergoes an initial rapid proliferation during infancy (proliferative phase) characterised by aggressive angiogenesis, followed by spontaneous involution over the next 1-5 years (involuting phase) and continued improvement up to 10 years (involuted phase), often with a fibro-fatty residuum. IH consists of cells of various lineages, with the presence of mesenchymal stem cells, endothelial progenitor cells, endothelial cells, myeloid haematopoietic cells, and pericytes. This thesis demonstrates the expression of primitive (stem/progenitor cell) markers on the endothelium of $\mathrm{IH}$. The expression of the transcription factors brachyury, Tal-1 and GATA-2, along with the demonstration of erythropoiesis in IH explants in vitro supports the hypothesis that $\mathrm{IH}$ consists of a primitive endothelium similar to an embryonic haemogenic endothelium. The expression of the erythropoietin receptor and haemoglobin zeta chain by the endothelium of $\mathrm{IH}$ further strengthens the notion that $\mathrm{IH}$ is a haemogenic endothelium. Consistent with the primitive embryonic origin, the expression of the placental markers human chorionic gonadotrophin (hCG) and human placenta lactogen (hPL), but not cytokeratin 7 (CK7) or human leucocyte antigen- $\mathrm{G}(\mathrm{HLA}-\mathrm{G})$ by the endothelium in IH, supports a placental chorionic villous mesenchymal core cell, and not a trophoblast, origin for $\mathrm{IH}$. IH thus has an extraembryonically derived primitive mesodermal origin. This primitive mesoderm is able to account for the haemogenic endothelium phenotype of the endothelium of 
proliferating $\mathrm{IH}$ microvessels with its capacity for both erythropoietic and mesenchymal differentiation.

Additionally, data are presented to show that IH expresses key components of the renin-angiotensin system (RAS), angiotensin converting enzyme (ACE), angiotensin II (ATII), angiotensin receptor 2 (ATR2). Cultured IH-derived stem cells can be induced to proliferate and form blast colonies in response to ATII treatment. The crucial regulatory role of RAS in the proliferation and differentiation of the stem/progenitor cell population within $\mathrm{IH}$ accounts for the natural progression of $\mathrm{IH}$. A model is proposed to provide a rational explanation for the serendipiditous discovery of the dramatic effect that the $\beta$-blocker, Propranolol has in accelerating involution of $\mathrm{IH}$. The hypothesis that Propranolol exerts its action on $\mathrm{IH}$ through modulation of the RAS by blocking renin activity and preventing the conversion of angiotensinogen to angiotensin I, thereby reducing ATII levels, has led to a clinical trial using Captopril, an ACE inhibitor in the treatment of problematic proliferating $\mathrm{IH}$. The observed accelerated involution of $\mathrm{IH}$ by Captopril which blocks the conversion of angiotensin I to ATII confirms a key regulatory role for RAS in the biology of IH This discovery underpins the development of potentially safer and novel treatment modalities for this enigmatic condition.

Parts of this work are published as follows:

Itinteang, T., Tan, ST., Brasch, H. \& Day, DJ. Primitive mesodermal cells with a neural crest stem cell phenotype predominate proliferating infantile haemangioma. $J$ Clin Pathol 63, 771-776 (2010).

Itinteang, T., Tan, ST., Brasch, H. \& Day, DJ. Haemogenic endothelium in infantile haemangioma. J Clin Pathol 63, 982-986 (2010). 
Tan, ST., Itinteang, T. \& Leadbitter, P. Low-dose propranolol for infantile haemangioma. J Plast Reconstr Aesth Surg 64, 292-299 (2011).

Itinteang, T., Vishvanath, A., Day, D.J. \& Tan, S.T. Mesenchymal stem cells in infantile haemangioma. J Clin Pathol 64, 232-236 (2011).

Itinteang, T., Tan, ST., Brasch, HD., Vishvanath, A. \& Day, DJ. Primitive erythropoiesis in infantile haemangioma. Br J Dermatol 164, 1097-1100 (2011).

Itinteang, T., Brasch, H.D., Tan, S.T. \& Day, D.J. Expression of components of the renin-angiotensin system in proliferating infantile haemangioma may account for the propranolol-induced accelerated involution. J Plast Reconstr Aesth Surg 64, 759-765 (2011).

Tan, ST., Itinteang, T. \& Leadbitter, P. Low dose propranolol for mutiple infantile hepatic and cutaneous hemangiomas with deranged liver function. Pediatrics 127, e1-e5 (2011).

Itinteang T., Withers, AHJ., Leadbitter, P., Day, DJ \& Tan, ST. Pharmacologic therapies for infantile hemangioma: is there a rational basis. Plast Reconstr Surg 128, 499-507 (2011).

Vishvanath, A., Itinteang, T., Tan, ST. \& Day, DJ. Infantile haemangioma expresses tumour necrosis factor-related apoptosis-inducing ligand (TRAIL), TRAIL receptors, osteoprotegerin and receptor activator for nuclear factor ligand (RANKL). Histopathology 59, 397-406 (2011). 
Itinteang, T., Tan, ST., Guthrie, S., Tan, CES., Mclntyre, BC., Brasch, HD. \& Day, DJ. A placental chorionic villous mesenchymal core cellular origin for infantile haemangioma. J Clin Pathol 64, 870- 874 (2011).

Parts of this work were awarded the following prizes;

$18^{\text {th }}$ International Workshop on Vascular Anomalies, Brussels, Belgium- John B Mulliken Prize 2010.

Best Scientific Paper award- at the 2010 NZ Association of Plastic Surgeons Annual Scientific Meeting, Auckland, NZ.

Gold Award for the best student publication in Cell \& Molecular Biology, School of Biological Sciences, Victoria University of Wellington, Wellington, NZ- 2010.

2010 Postgraduate Research Excellence Award category winner, Victoria University of Wellington. Wellington, NZ.

$16^{\text {th }}$ Congress International Confederation for Plastic Reconstructive and Aesthetic Surgery, Vancouver, Canada- International Award for the best Basic Science Presentation by a Resident in 2011 .

Emmett Prize for the best Research Presentation at the SET 2-5 Plastic \& Reconstructive Registrars' Conference, Brisbane, Australia 2012. 


\section{Chapter 1: Introduction}

\subsection{Infantile Haemangioma}

Vascular anomalies (VA) are localised defects in the structure of the vasculature. There has been considerable confusion in the terminology in the literature leading to inappropriate treatment and misdirected research efforts. The term haemangioma has been used to describe all the forms of vascular anomalies. In 1982 Mulliken and Glowacki ${ }^{1}$ proposed a classification of vascular anomalies, based on biology, into haemangioma and vascular malformations. This classification was adapted by the International Society for the Study of Vascular Anomalies (ISSVA) in 1996.

Haemangioma, is the most common tumour of infancy (Figure 1.1), commonly referred to as infantile haemangioma $(\mathrm{IH})$ or colloquially, strawberry birthmarks. It is a tumour of the microvasculature, typified by aggressive angiogenesis ${ }^{1}$.

\subsubsection{Incidence}

$\mathrm{IH}$ affects approximately $10 \%$ of Caucasian infants ${ }^{2,3}$ with a lower incidence in dark skin races. It is more common in Caucasian, female, and in premature infants $^{2,4}$. The increased incidence of $\mathrm{IH}$ in these sub-populations is unexplained.

\subsubsection{Clinical Features of Infantile Haemangioma}


Most IHs are not present at birth with $40 \%$ evident in the first two weeks of life. $\mathrm{IH}$ typically presents as a small red mark or an area of telangiectasia with a surrounding pale halo and undergoes rapid proliferating within the first year of life (proliferative phase) $)^{1}$. This is followed by a spontaneous involution over the next five years (involuting phase), with on-going improvement up to 10 years (involuted phase) usually leaving a fibrofatty residuum (Figure 1.2) $2,5,6$ (Figure $1 \mathrm{~A}-\mathrm{D}$ ). The endothelium of $\mathrm{IH}$ expresses the immunohistochemical marker glucose transporter-1 (GLUT-1) that distinguishes IH from other forms of vascular anomalies ${ }^{6}$. Sixty percent of $\mathrm{IH}$ occur in the head and neck region, with lesions in the trunk and extremities making up for 25 and 15 percent respectively.
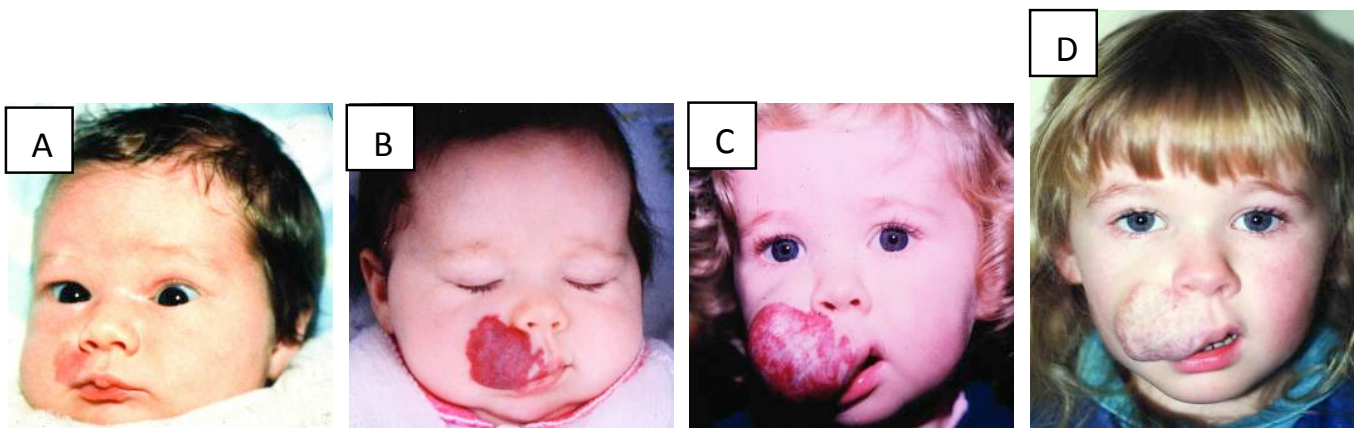

Figure 1.1. Serial images of a child with a large facial $\mathrm{IH}$ at 2 weeks $(A), 1$ month (B), 6 months (C) and 6 years (D) of age (Courtesy of Professor Swee T. Tan).
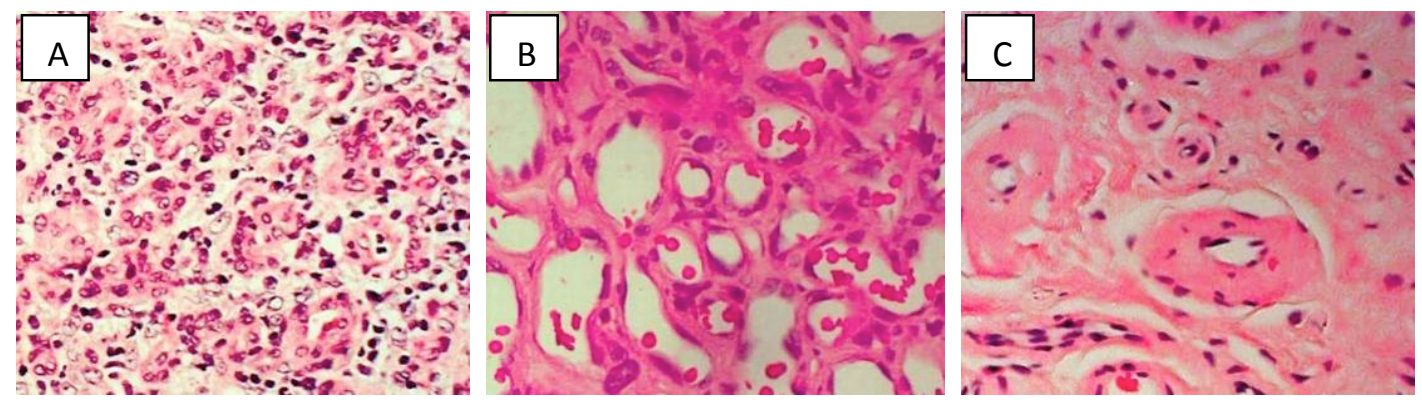
Figure 1.2. Haematoxylin and eosin staining of representative IH sections taken during proliferation $(A)$, involuting $(B)$ and involuted $(C)$ phases demonstrating the diminishing cellularity as IH involutes (Courtesy of Professor Swee T. Tan).

\subsubsection{Subtypes of Infantile Haemangioma}

$\mathrm{IH}$ commonly presents as discrete localised lesions mostly affecting the skin and/or subcutaneous tissues ${ }^{7}$. It may involve any other tissues or organs such as liver, spleen, gut, lung, bladder and adrenal glands ${ }^{8}$. Multiple cutaneous lesions are not uncommon ${ }^{9}$, with an increased risk of hepatic involvement with increasing number of cutaneous lesions ${ }^{9,10}$.

Focal lesions present as localised lesions mostly affecting the face ${ }^{7}$ (Figure 1.3). Segmental lesions are generally more extensive and plaque-like. The segmental distribution of these IHs corresponds to the embryological mesenchymal prominences of the head and neck development, i.e., frontonasal, maxillary or mandibular ${ }^{11}$ (Figure 1.4).

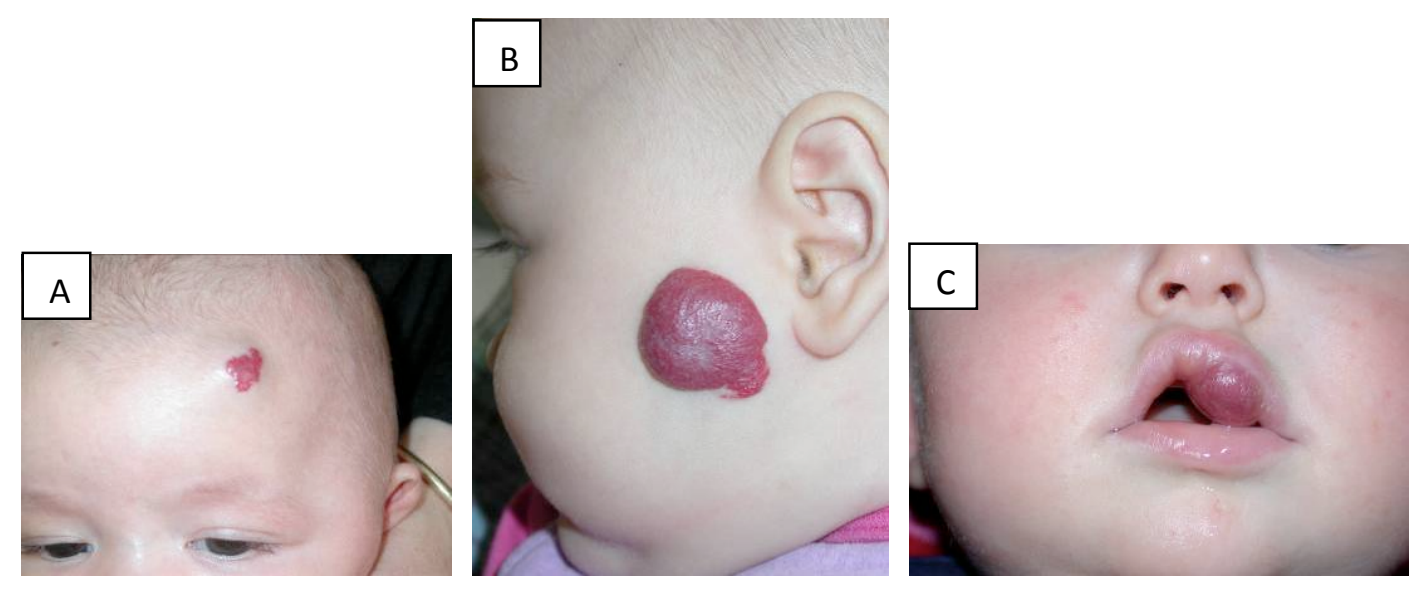


Figure 1.3. Localised focal IH lesions on the forehead (A), left cheek (B) and left upper lip (Courtesy of Professor Swee T. Tan).
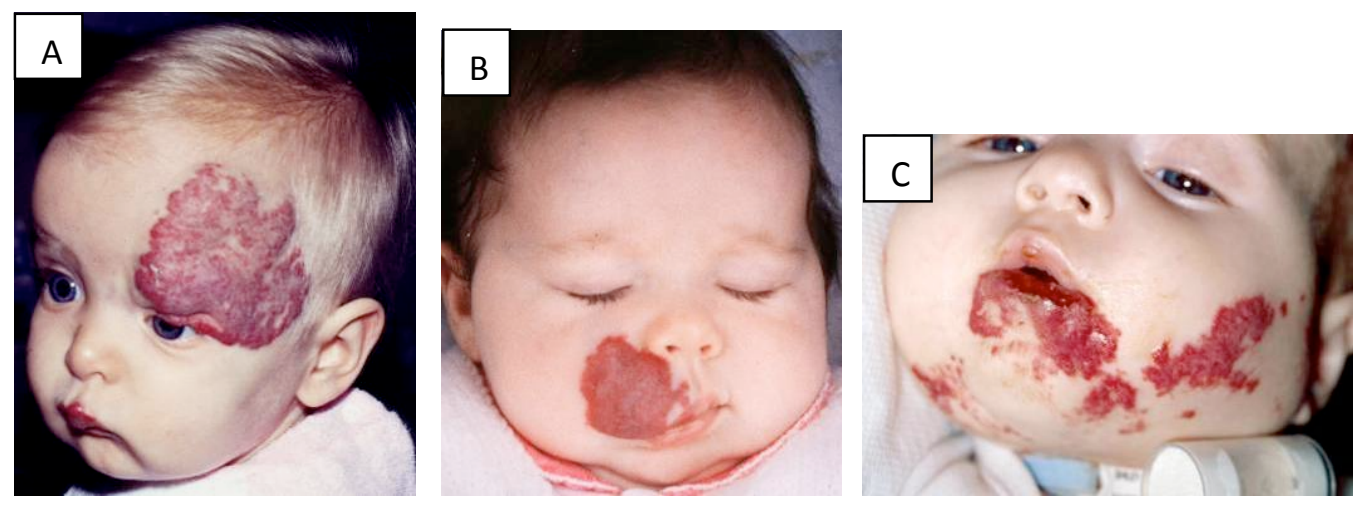

Figure 1.4. Segmental IHs in the left frontal $(A)$, right maxillary $(B)$, and mandibular

(C) segmental distributions.

\subsubsection{PHACES Syndrome}

PHACES syndrome is a neurocutaneous syndrome affecting a sub-group of patients with segmental IHs, in which the segmental IH is associated with one or more of the following features: Posterior fossa malformations of the brain, arterial anomalies, cardiac anomalies, aortic coarctation, eye abnormalities, sterna cleft and/or supraumbilical raphe (Figure 1.5) ${ }^{12,13}$. The observed subtype of $\mathrm{IH}$ and in particular, the association with midline structural anomalies are unexplained. 


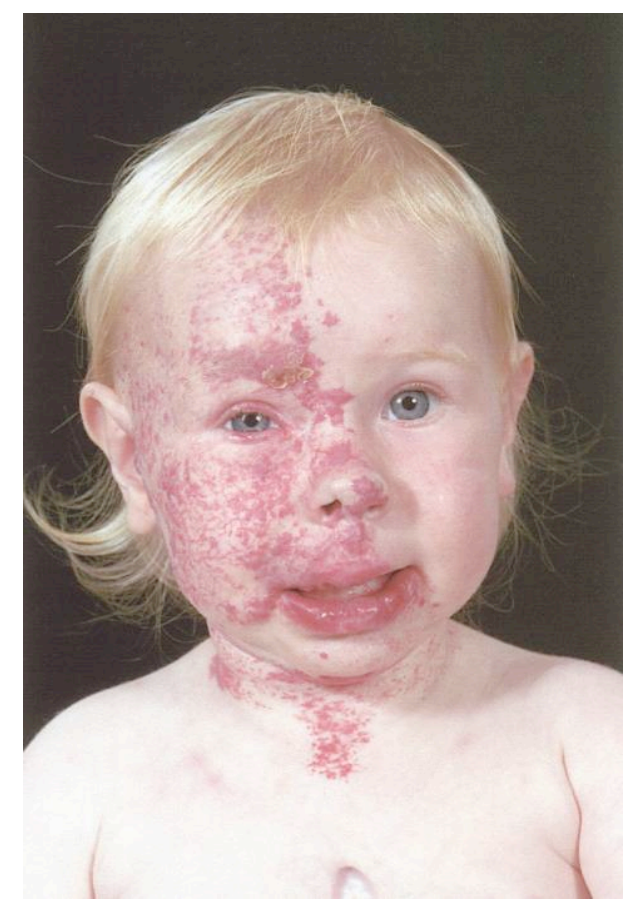

Figure 1.5. A child with PHACES syndrome demonstrating segmental facial IH with an associated sternal cleft (Courtesy of Professor Swee T. Tan).

\subsubsection{Congenital haemangiomas}

There is a small subgroup of IHs that are fully grown at birth and they have been called congenital haemangiomas ${ }^{14,15}$. Congenital haemangiomas are further sub-categorised into (1) Rapidly involuting congenital haemangioma $(\mathrm{RICH})^{15}$, which regresses completely by $10-14$ months of age, and (2) Noninvoluting congenital haemangioma $(\mathrm{NICH})^{14}$, which never regresses and grows proportionally with the child. $\mathrm{RICH}$ and $\mathrm{NICH}$ share similar histological features with the common IH. However, unlike IH they do not express GLUT$1^{14,15}$, a marker for $\mathrm{IH}$. 


\subsubsection{Complications and Indications for Treatment of Infantile Haemangioma}

Approximately $50 \%$ of $\mathrm{IH}$ will regress by age five and all be age $10^{16}$ with approximately $25 \%$ requiring surgery and or laser therapy during the involuting and involuted phases ${ }^{17}$. The self limiting and benign progression of the majority of $\mathrm{IH}$ renders an expectant management approach being adopted. However, $10-14 \%$ of $\mathrm{IH}$ require active intervention during the proliferative phase because of its threat to life or function or tissue distortion or destruction ${ }^{18,19}$ as the result of the involvement of critical organs, such as the airways, eyes, liver, and ulceration.

The heterogeneity and innate tendency for $\mathrm{IH}$ to involute provides an exciting and equally challenging dilemma for the physician caring for infants with $\mathrm{IH}$. It is the ability to predict those patients in need of treatment or with the highest risk of a complication that provides the greatest challenge for physicians. A study in 2006 of over one thousand patients with $\mathrm{IH}$ revealed that morphological subtype and anatomical location were by far the greatest predictors of complication and the requirement for treatment ${ }^{20}$. Of the morphologic subtypes, segmental IHs were by far more likely to require treatment compared to localised lesions, by a factor of greater than 10 . When accounting for anatomical location, perineal IHs were noted to have the highest rate of ulceration, although fortunately this location is relatively uncommon. Facial IH on the other hand represents the most common location for $\mathrm{IH}$, accounting for a proportionately higher rate of the need for active 
treatment ${ }^{20}$. Table 1.1 shows the IHs with the highest risk for the need of intervention $^{19}$.

\begin{tabular}{|c|c|}
\hline Anatomic Locatlon/Morphology & Assoclated Risk \\
\hline Faclal, large segmental & $\begin{array}{l}\text { PHACES syndrome (posterior fossa malformations, hernangiomas, } \\
\text { arterial anomalles, cardlac defects, eye abnormalities, sternal } \\
\text { defting) }\end{array}$ \\
\hline $\begin{array}{l}\text { Nasal tip, ear, large facial (especially with prominent } \\
\text { dermal component) }\end{array}$ & Permanent scarring, disfigurement \\
\hline Periorbital and retrobulbar & Ocular axis occlusion, astigmatism, amblyopla, tear-duct occlusion \\
\hline Segmental "beard area," central neck & Alrway hernangloma \\
\hline Perioral & Ulceration, disfigurement, feeding difficulties \\
\hline Segmental overlying lumbosacral spine & Tethered spinal cord, genitourinary anomalies \\
\hline Perineal, axlla, neck, perioral & Ulceration \\
\hline Multiple hemangiomas & Visceral Inwolvement (espectally Iver, gastrointestinal tract) \\
\hline
\end{tabular}

Table 1.1. Features of $\mathrm{IH}$ with the highest risk of morbidity according to anatomic location and morphology of the haemangioma (Adapted from Chang, et al. ${ }^{19}$ ).

\subsubsection{Treatment of Infantile Haemangioma}

It is noteworthy that the identification of chemotherapeutic agents suitable for the treatment of problematic IH has predominantly been serendipitously discovered.

The mainstay treatment for problematic $\mathrm{IH}$ is pharmacological with high dose corticosteroids being first line agents ${ }^{21}$. More recently vincristine ${ }^{22}$ has replaced interferon ${ }^{23}$ as second line treatment in cases that failed corticosteroid therapy. However, since their serendipitous discovery, $\beta$ blockers ${ }^{24,25}$ have now surpassed corticosteroids as the first choice of treating problematic $\mathrm{IH}$ in many centres ${ }^{26,27}$. Recent use of propranolol and a review 
on the treatment and future possible therapies for $\mathrm{IH}$ is discussed in depth in Chapter 11 of this thesis.

\subsection{Cellular Elements of Infantile Haemangioma}

\subsubsection{Endothelial Cells}

$\mathrm{IH}$ has traditionally been regarded as a benign tumour of the microvasculature with proliferating lesions composed of immature microvessels, that are lined by plump, mitotically active endothelial cells, characterised by the expression of endothelial markers (CD31, vWF, Ulex europaes, and CD34) 2,28 .

Cells that are phenotypically similar to endothelial cells have been isolated from proliferating $\mathrm{IHs}$ and characterised and their similarities to endothelial cells isolated from second trimester fetal tissues have been observed $^{29}$. Other studies have suggested a clonal origin for the endothelial cells in $\mathrm{IH}^{30}$.

\subsubsection{Myeloid Haematopoietic Cells}

Post-natally, all haematopoietic cells are generated in the bone marrow from pluripotent haematopoietic stem cells, where the lymphoid and myeloid lineages diverge. Myeloid progenitors generate granulocyte/macrophage progenitors in the marrow and upon entering the circulation, forms downstream myeloid lineages known as the polymorphonuclear leucocytes, monocytes and mast cells ${ }^{31}$. 
The abundance of mast cells within IH has been noted to be predominantly localised to the interstitium, and the numbers tended to increase as the tumour involuted ${ }^{4}$. The role of mast cells in the involution of $\mathrm{IH}$ has been attributed to their production of pro-apoptotic proteins during involution ${ }^{4}$. Fig. 1.6 shows mast cells located within the interstitium of a proliferating $\mathrm{IH}$.

It is interesting that another study identifying haematopoietic leucocytes also noted interstitial localisation of this population within $\mathrm{IH}$ with a proportional decrease during involution, suggesting a role for secretion of cytokines $^{32}$ from these myeloid cells during $\mathrm{IH}$ progression.

The source of these myeloid cells seen in abundance within $\mathrm{IH}$ is unknown and is presumed to have recruited from the bone marrow via the circulation $^{4,32,33}$.

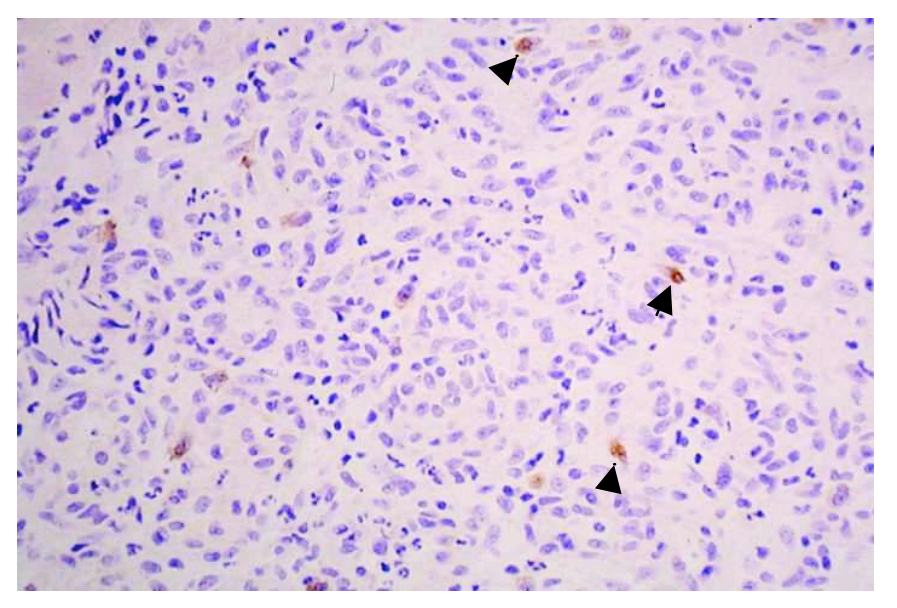

Figure 1.6. Mast cells in proliferating $\mathrm{IH}$. Mast cells were identified in $\mathrm{IH}$ by counterstaining for chymase (brown DAB staining). The brown cells (arrows) are located in the interstitium. The section is counter stain with eosin (Courtesy of Professor Swee T. Tan). 


\subsubsection{Pericytes}

Pericytes are mural cells proposed to originate post-natally in the bone marrow/stroma which are recruited into areas of angiogenesis to form the pericytes, to provide support for the endothelial cells.

Pericytes have been characterised by their expression of smooth muscle actin $(\mathrm{SMA})^{34}$. Immunohistochemical $(\mathrm{IHC})$ studies of $\mathrm{IH}$ have revealed an inner cell layer characterised by the expression of endothelial associated proteins and an outer concentric pericyte layer that expresses $\mathrm{SMA}^{2,28}$.

\subsubsection{Adipocytes}

Adipocytes form the predominant cell type within the fibrofatty residuum in involuted $\mathrm{IH}^{5}$. The origin of these adipocytes is unknown.

\subsection{Stem Cell Theories of the Origin of Infantile Haemangioma}

\subsubsection{Endothelial Progenitor Cells}

Endothelial progenitor cells (EPCs) have been deemed haematopoietic progenitor cells, generated in the bone marrow and possessing the innate ability to differentiate into cells of the endothelial lineage ${ }^{35}$. EPCs have been characterised by the expression of CD133, CD34 and VEGFR-2 ${ }^{35}$. Whether 
these EPCs exist as a distinct entity within the spectrum of haematopoieticendothelial differentiation, or whether they simply reflect a transitional state remains unresolved.

EPCs have been identified within proliferating $\mathrm{IH}^{36}$, and there is an increased level of these cells in the circulation of affected patients ${ }^{37}$. These EPCs have been proposed to be the precursors to the endothelial cells that predominate in proliferating lesions. These EPCs have been proposed to originate from the bone marrow and recruited into $\mathrm{IH}$. However, the chemical signals associated with this migration pattern remain elusive ${ }^{36}$.

\subsubsection{Mesenchymal Stem Cells}

Mesenchymal stem cells (MSCs), more appropriately called mesenchymal progenitor cells (MPCs), have been characterised by three main properties including: (i) the expression of mesenchymal associated proteins such as CD29, CD44, CD90, and vimentin, (ii) the adherence to plastic in culture, and (iii) the ability to differentiate and form terminal mesenchymal derived cells such as adipocytes, osteoblasts and chrondrocytes ${ }^{33}$. True MPCs do not express endothelial or haematopoietic associated markers ${ }^{33,38}$.

The presence of a mesenchymal progenitor population within proliferating $\mathrm{IH}$ was demonstrated by $\mathrm{Yu}$ et $\mathrm{a}^{39}$. They based the isolation of these cells predominantly on their adherence to plastic. Yu's report also highlighted the characterisation of these MPCs following at least ten passages in culture, and revealed the expression of mesenchymal-only associated 
proteins, with the exclusion of haemato/endothelial markers. They were also able to demonstrate adipocyte and osteoblast differentiation capacity in this cell population. It has been suggested that these mesenchymal progenitors which give rise to the fatty tissue seen during involution, have been recruited into the $\mathrm{IH}$ either from the bone marrow or adjacent niches ${ }^{39}$.

\subsection{Neural Crest Cells}

Neural crest cells are bilaterally paired epithelial cells arising from the ectoderm at the margins of the neural tube. These cells undergo an epithelialmesenchymal transition (EMT), such that they undergo a change in phenotype and transform into migratory mesenchymal cells, which migrate to many locations and differentiate into different cell types in the embryo ${ }^{40}$.

\subsubsection{Segmental Migration and Its Role in Embryogenesis}

There are two distinct patterns of neural crest cell migration relating to anatomical location. In the cephalic region, neural crest cells migrate into the pharyngeal arches forming tissues of ectomesenchymal origin, and contribute to the formation of the facial skeleton, fat, connective tissue, teeth, melanocytes and peripheral neurons $\mathrm{s}^{41,42}$.

In contrast, in the trunk region, neural crest cells contribute to the peripheral and autonomic nervous system (Figure 1.7). 


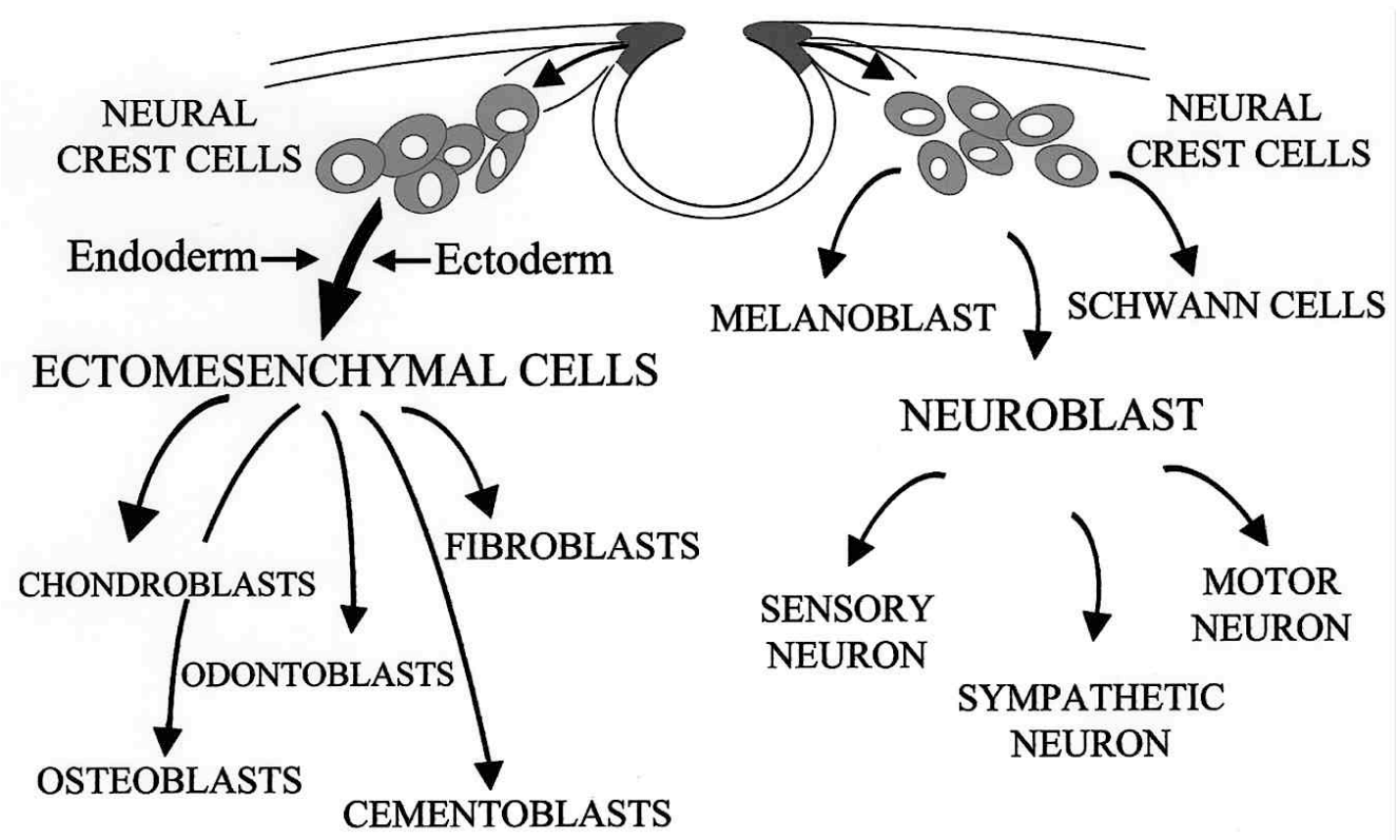

Figure 1.7. Illustration of the lineage differentiation capacity of neural crest cells (Adapted from Chai, et al. ${ }^{41}$ ).

\subsubsection{Characterisation and Identification}

Neural crest cells are characterised by the expression of various cell surface and cytosolic proteins. Three such neural crest associated proteins namely p75, Sox 9 and Sox 10 are discussed in this thesis. Low-affinity nerve growth factor receptor (LNGFR), also known as neurotropin/p75, is a cell surface marker typically identifying neural crest cells. Sox 9 and Sox 10, are members of the group E Sox genes, which play an important role in the development of neural crest cells both at the induction of neural crest cell stage and at the migratory stage $\mathrm{e}^{43}$. It is relevant that Sox 9 is also expressed in many other cell types in addition to neural crest cells, such as chrondrocytes ${ }^{44}$. 


\subsubsection{Embryonic Stem Cell Origin of Neural Crest Cells}

Human embryonic stem cells (hESCs) have provided an invaluable in vitro model for studying human development including the induction and differentiation of the nervous system.

A recent report by Lee et $\mathrm{al}^{45}$, shows that the differentiation and isolation of human neural crest stem cells from a hESC line involved the addition of fibroblast growth factor 2 or bone morphogenic protein $2^{45}$. It is exciting that the authors note the neuronal differentiation capacity of these ESC-derived neural crest cells, which can further differentiate into downstream neurons through the addition of cytokines of which nerve growth factor (NGF) and the second messenger cyclic AMP play a critical role. It is interesting that the addition of serum to the culture medium of these neural crest stem cells induces their differentiation into mesenchymal cells. Figure 1.8 , summarises the findings of the bi-potent nature of these ESC-derived neural crest cells. This dual potential of ESC-derived neural crest cells strikes parallels with neural crest cells derived from IH tissues, and highlights the similarities between these populations, which will be the topic of investigation in this thesis. The differentiation capacity of mesenchymal cells to form terminal mesenchymal tissues such as adipocytes, and osteoblasts has been discussed earlier in this chapter. 


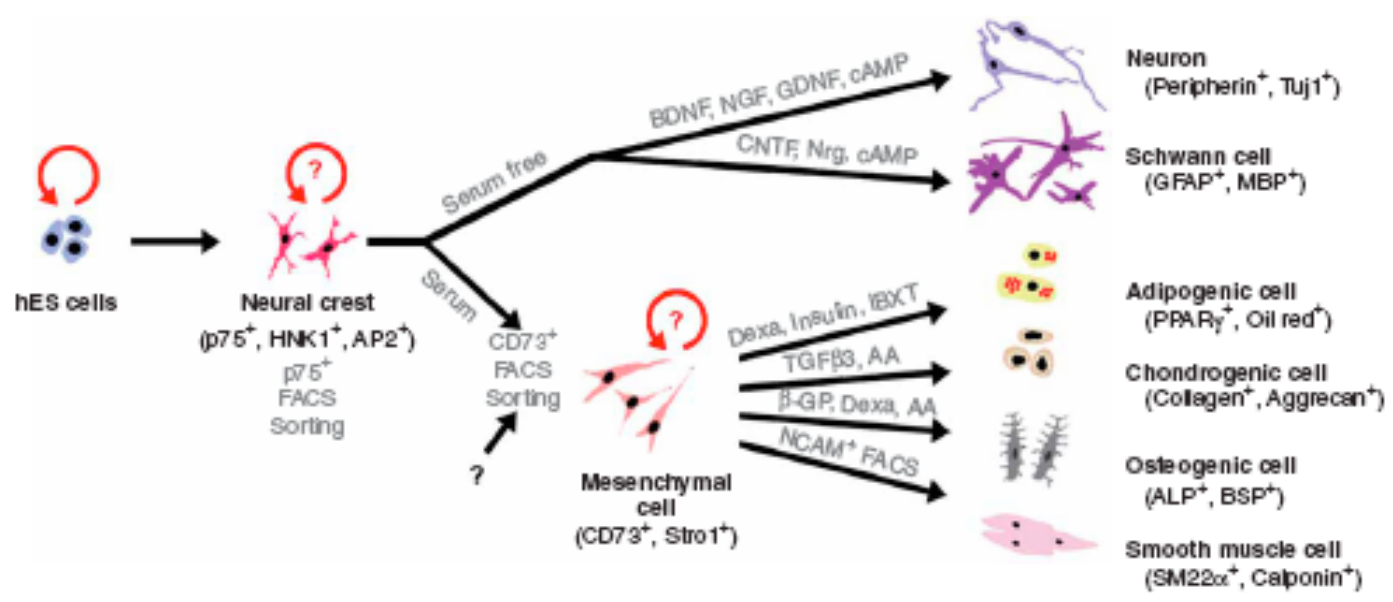

Figure 1.8. Shows lineage characterisation and differentiation of ESC-derived neural crest stem cells with the ability to form downstream neuronal and mesenchymal derivatives (Adapted from Lee, et al. ${ }^{45}$ ). 


\subsection{Osteoprotegerin}

Osteoprotegerin (OPG) is a secreted glycoprotein belonging to the tumour necrosis factor (TNF) receptor family, and has been identified in both the tumour necrosis factor-related apoptosis-inducing ligand (TRAIL) and (receptor for nuclear factor $\mathrm{\kappa B}$ ligand (RANKL) pathways ${ }^{46}$. TRAIL, also known as Apo2L, and its soluble decoy receptor, OPG, have been implicated in the pathogenesis of $\mathrm{IH}^{11}$. TRAIL, acting via receptors, namely death receptors 4 and 5 (DR4 and DR5) induce cells to undergo apoptosis. However, binding of TRAIL to its decoy receptors, DcR1 and DcR2, prevents apoptosis. We have previously demonstrated the presence of OPG on the immature capillaries of proliferating $\mathrm{IH}$, which is lost as the microvessels mature. We postulate that OPG acting through the TRAIL pathway prevents cells of the immature capillaries of $\mathrm{IH}$ from undergoing apoptosis ${ }^{11}$.

\subsubsection{Osteoprotegerin and Receptor for Nuclear Factor $\kappa \mathrm{B}$ Ligand}

OPG has been shown to be a soluble decoy receptor for TRAIL, as well as a regulator of bone remodelling through its action on the receptor for RANKL. RANKL is also a member of the TNF superfamily and is expressed in the bone marrow environment ${ }^{47}$. RANKL is the ligand for its receptor RANK, which is primarily expressed on osteoclast precursors. The binding of RANKL to RANK receptors triggers osteoclast recruitment and differentiation. OPG on the other hand has been demonstrated to bind to RANKL and inhibits the 
interaction of RANKL with RANK, thus inhibiting downstream osteoclastic bone resorption ${ }^{47}$.

\subsection{Haematopoiesis}

The haematopoietic system in humans is one of the first major organs to form, because of its crucial role in supplying oxygen to the growing foetus ${ }^{48}$. The haematopoietic system consists of a series of hierachical regulation of pluripotent haematopoietic stem cells down to functionally terminally differentiated and distinct blood cells ${ }^{49}$. It is this regulation of differentiation and proliferation of haematopoietic stem cells to eventual downstream terminally differentiated blood cells that embodies the process of haematopoiesis.

\subsubsection{Human Haematopoiesis}

In humans the haematopoietic stem cell pool is already formed during embryogenesis. There are two waves of embryonic haematopoiesis namely the primitive and the definitive haematopoietic waves in corresponding chronological order ${ }^{48}$.

\subsubsection{Primitive Haematopoiesis}

Primitive, or ventral, haematopoiesis occurs outside the embryo, i.e., extraembryonic, in the yolk sac from around day 17 to around day 40 of gestation (Figure 1.9 $)^{50,51}$. This extra-embryonic haematopoietic process involves the 
initial aggregation of primitive mesoderm cell aggregates, the internal cells simultaneously disappearing to form the first open vessel lumen with a few primitive mesodermal cells adhering to the de-novo endothelium to form structures known as blood islands ${ }^{50}$. During this early stage of haematopoiesis erythoid cells predominant, with reports of megakaryocytes and macrophages also being present.

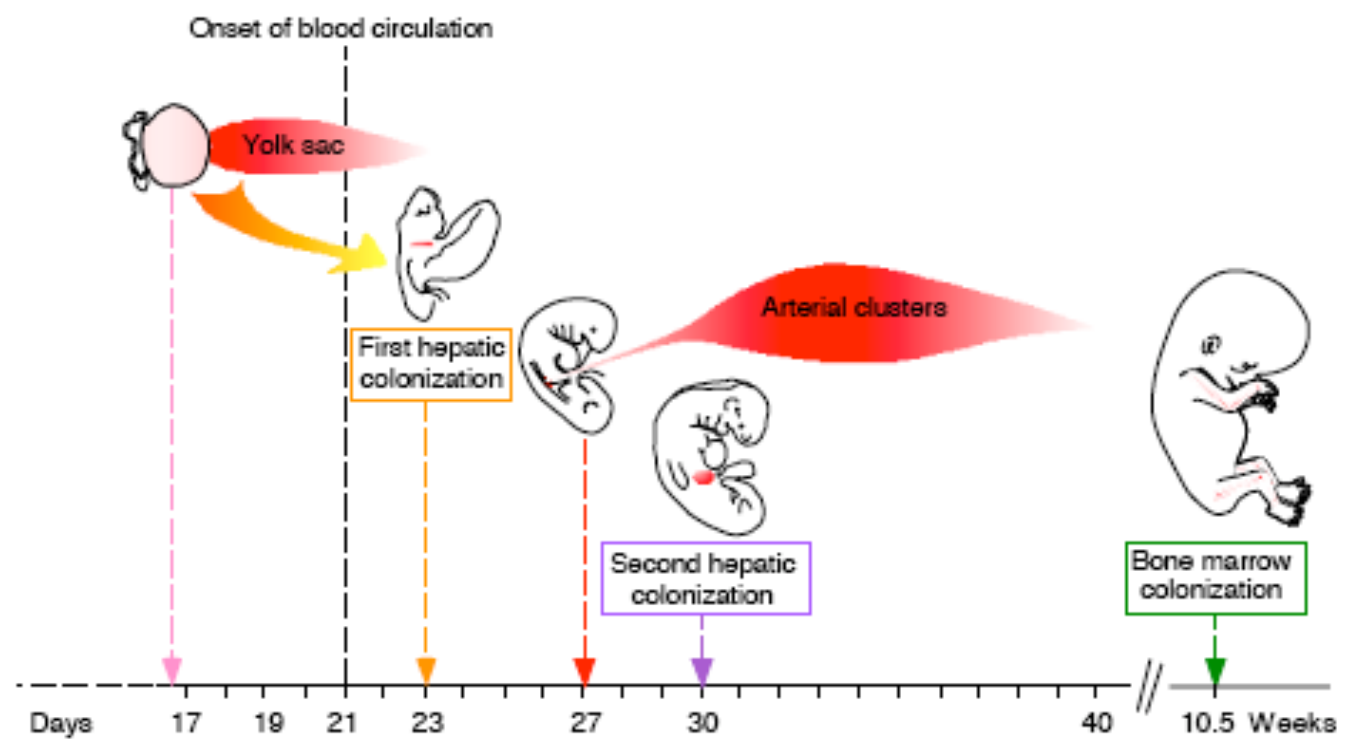

Figure 1.9. The chronological appearance of haematopoietic sites in the human embryo (Adapted from Tavian, et al. ${ }^{50}$ ).

\subsubsection{Definitive Haematopoiesis}

The onset of definitive haematopoiesis coincides with the onset of the cardiac rhythm and hence the onset of the circulation ${ }^{51}$. The onset of the circulation allows the primitive yolk-sac derived blood cells to enter the embryonic tissues and therefore colonise the embryonic sites of haematopoiesis. It is at about the day 23 stage that the liver is initially colonised and forms the first major 
organ for definitive haematopoiesis, and the first major haematopoietic organ in the foetus, but the role for the embryonic liver to function as a haematopoietic organ has been attributed to a second wave of colonisation that takes place at the day 30 stage $^{50}$.

It is interesting to note that from day 27 a transient site of intraembryonic haematopoiesis has also been demonstrated in the umbilical region of the aorta and the vitelline artery. This site of haematopoiesis becomes prominent at day 35 , with clusters of thousands of cells clearly visible, which then disappear after day 40 gestation ${ }^{50}$.

Bone marrow haematopoiesis is the main adult haematopoietic forming tissue, which starts during the $11^{\text {th }}$ week of gestation. Haematopoiesis in the bone marrow develops within mesodermal structures, known as logettes. These logettes are composed of a network of mesenchymal cells supported by dense fibrillar material surrounding a central artery (Figure 1.10$)^{52}$. The earliest progeny that differentiate from the bone marrow are $\mathrm{CD} 15^{+}$myeloid and glycophorin $\mathrm{A}^{+}$erythroid cells ${ }^{50}$.

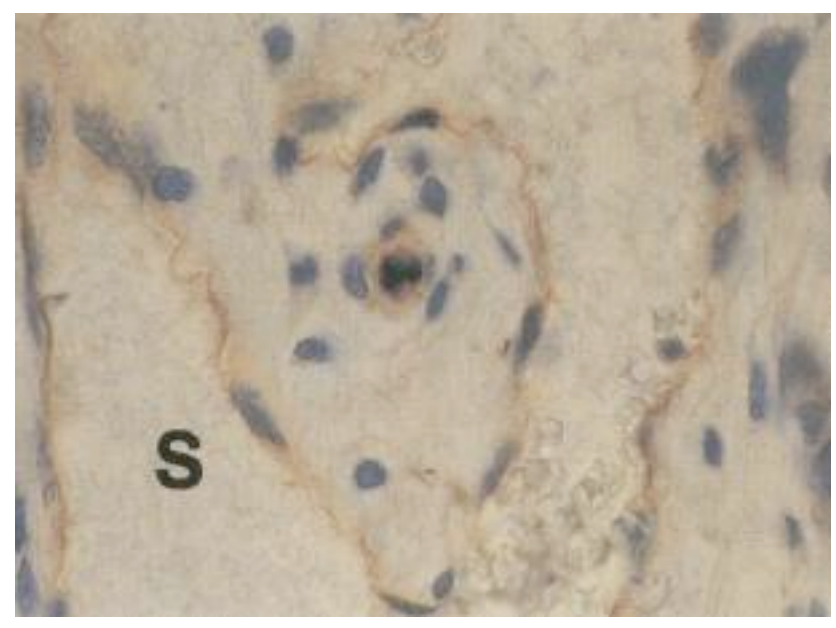


Figure 1.10. IHC slide showing staining of a logette for CD34+ endothelial cells which are surrounded by CD34-/SMA+, and a surrounding sinus (S) lined by CD34+ endothelium (Adapted from Charbord, et al ${ }^{52}$ ).

\subsubsection{Haemogenic Endothelium}

The first site of mammalian intra-embryonic haematopoiesis is in the region of the dorsal aorta. As stated earlier, the lack of an in vivo human model means that most of these studies have been performed on murine models of development ${ }^{50}$. This period of development in the mouse embryo at E10.5 11.5 is characterised by the developing aorta becoming flanked by the gonado-mesonephric columns, and hence the region has been appropriately referred to as the Aorto-Gonado-Mesonephros (AGM). It is during this period of gestation that haematopoietic cells can be seen budding off from the aorta, and it is this concept of haematopoietic cells generated from an endothelial phenotype that forms the basis of the concept of the haemogenic endothelium ${ }^{53}$. Although there is some debate as to whether these haematopoietic cells are indeed derived from the endothelium or from its underlying mesenchyme ${ }^{54}$,a report by Zovein et $a^{55}$ confirms, through lineage tracing, the endothelial origin of these haematopoietic cells.

\subsubsection{Haemangioblast}

The notion of a common precursor to both cells of the endothelial and haematopoietic lineages, has formed the basis for the hypothesis of a common precursor, the so called haemangioblast ${ }^{56}$. However, the direct in vitro demonstration of the haemangioblast was not achieved until $1998^{57,58}$. 
Subsequent studies have demonstrated the derivation of haemangioblasts from ESCs via a primitive mesoderm intermediate, with the ability to form dual lineages (Figure 1.11) $)^{59,60}$.

Time Course of

EB differentiation (day)

0

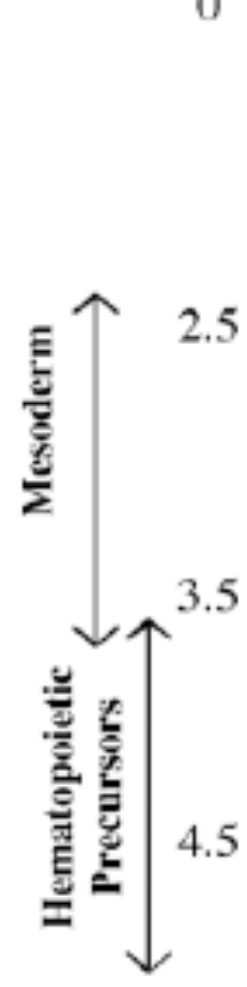

ES cells

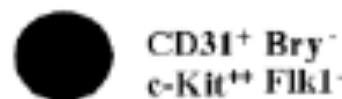

$\mathrm{c}=\mathrm{Kit}^{++}$Flk1

Pre-Mesoderm

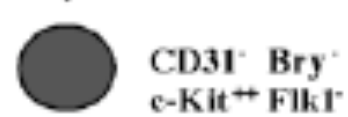

c-Kit ${ }^{++}$Flk1

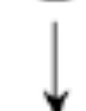

Pre-Hemangioblast

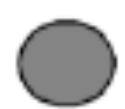

CD31. Bry ${ }^{++}$

e-Kit ${ }^{+}$Flk1

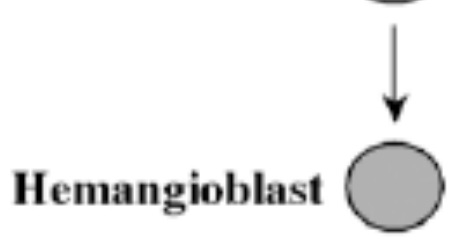

$\mathrm{CD}^{\prime} \mathrm{Bry}^{+}$

c-Kit: Flk1 ${ }^{*}$

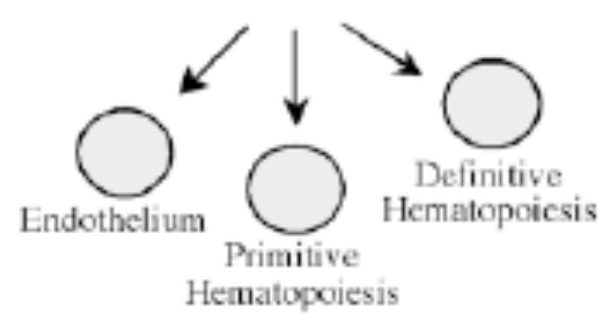

Figure 1.11. The ESC origin of the haemangioblast. Haemangioblast has the ability to differentiate and form downstream endothelial and haematopoietic lineages (Adapted from Fehling, et al. ${ }^{59}$ ). 


\subsubsection{Development of Haemoglobin}

The ontogeny of the development of haemoglobin in humans mirrors the changes in the sites of erythropoiesis during human development. Mirroring these switches are the changes in the globin gene expression, from embryonic $\left(\zeta_{2} \varepsilon_{2}\right)$ to foetal haemoglobin $\left(\alpha_{2} \bigvee_{2}\right)$ at around seven weeks gestation, followed by predominantly adult haemoglobin $\left(\alpha_{2} \beta_{2}\right)$ at birth (Figure $1.12)^{61-63}$.

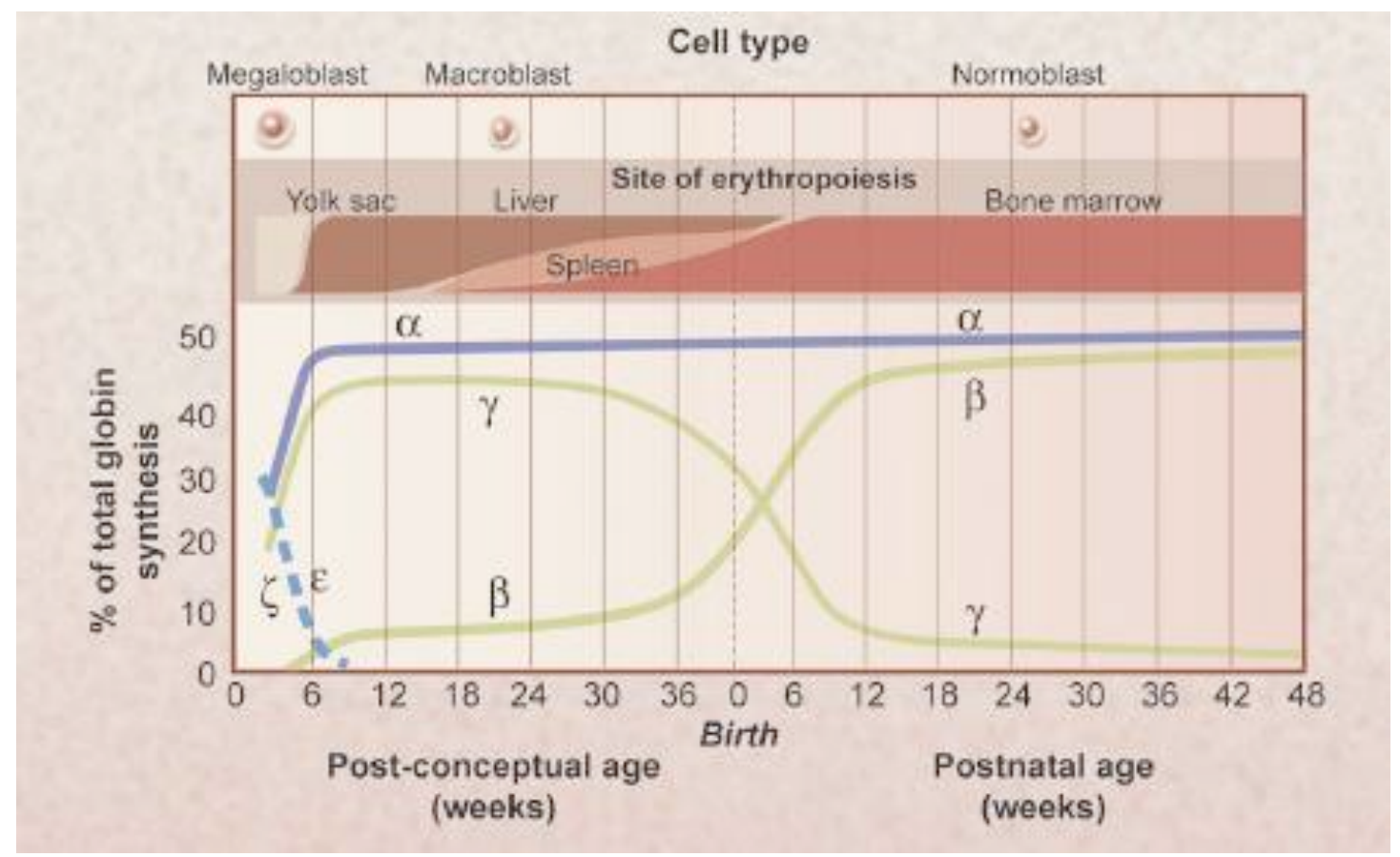

Figure 1.12. A schematic timeline for the development of human globin genes (Adapted from Schechter, et al. ${ }^{63}$ ). 


\subsection{Aims of this Thesis}

The aim of this thesis is to improve understanding of the cellular origin and development of $\mathrm{IH}$.

\subsubsection{Objectives}

The background and the key objectives for this study include:

(i) Preliminary data indicated that MSC may play an important role in the aetiology of $\mathrm{IH}$. This and other reports indicating primitive stem and progenitor cells in $\mathrm{IH}$ led us to investigate the presence and the phenotype of the stem cell populations present in $\mathrm{IH}$, and their role in $\mathrm{IH}$ progression.

(ii) Other studies in our laboratory have identified new putative therapeutic targets for $\mathrm{IH}$ treatment. This and the serendipitous discovery of propranolol as a treatment for $\mathrm{IH}$ led us to investigate the mechanism of propranolol action in inducing accelerated regression of $\mathrm{IH}$ and to identify other potential therapeutic targets/drugs for $\mathrm{IH}$. 


\section{Chapter 2: General Methods}

\subsection{Cell Culture}

\subsubsection{Infantile Haemangioma Tissue Specimens}

All IH tissue specimens used in this thesis were obtained from affected patients in accordance with a protocol approved by the Wellington Regional Ethics Committee. The IH specimens were then either fixed in $10 \%$ paraformaldehyde prior to wax embedding for immunohistochemistry analysis, or were used for tissue culture. Each specimen was categorised as either proliferating, involuting or involuted as previously described ${ }^{5}$. For this thesis a total of 12 proliferating and five involuted $\mathrm{IH}$ specimens were used with patients aged between 3-10 (mean 6) months and 6-9 (mean 7) years respectively.

\subsubsection{Cell Culture}

Plasticware and scalpels were obtained sterile from the manufacturers. Pipette tips, Eppendorfs, glass bottles, spatulae and forceps were appropriately autoclaved at $120^{\circ} \mathrm{C}$ for 20 mins prior to use. Handling of tissues and liquids were performed using aseptic techniques in a sterile Email Air Handling Class II Biological safety cabinet (AES Environmental Pty Ltd, Auburn, Australia) hereby mentioned as laminar flow hoods. 
All cell cultures were maintained in a Heracell incubator (Kendo Laboratory Products $\mathrm{GmbH}$, Hanau, Germany) at $37^{\circ} \mathrm{C}$ in humidified air and with $5 \% \mathrm{CO}_{2}$.

\subsubsection{Cell Lines}

\subsubsection{HeLa Cells}

HeLa cells were obtained from Dr Bronwyn Kivell, School of Biological Sciences, Victoria University of Wellington, and grown in Roswell Park Memorial Institute 1640 with glutamine (RPMI 1640 + glutamine, Gibco-BRL) medium supplemented with $10 \%$ foetal calf serum (FCS) and $2 \%$ Penstrep $(100 \mu \mathrm{g} / \mathrm{ml}$ streptomycin sulphate and $100 \mu \mathrm{g} / \mathrm{ml}$ Penicillin G, Gibco-BRL), henceforth referred to as cell culture medium. Cells were maintained in $75 \mathrm{~cm}^{2}$ sterile cell culture flasks (Greiner Bio-One) and passaged by splitting the cells in a ratio of $1: 3$ twice weekly.

\subsubsection{SY5Y Cells}

SY5Y cells were obtained from Ms Sushila Pillal, School of Biological Sciences, Victoria University of Wellington and maintained in a similar fashion as described above for HeLa cells. 


\subsubsection{EOMA Cells}

The mouse haemangioendothelioma (EOMA) cell line were obtained from $\mathrm{Dr}$ Anasuya Vishvanath, School of Biological Sciences, Victoria University of Wellington and maintained in a similar fashion as described above for HeLa and SY5Y cells.

\subsubsection{Cell Culture Techniques}

As required, cells frozen at $-80^{\circ} \mathrm{C}$ were routinely thawed in a water bath at $37^{\circ} \mathrm{C}$ prior to transferring to $15 \mathrm{ml}$ centrifuge tubes (Greiner Bio-one) containing $10 \mathrm{mls}$ of pre-warmed cell culture medium followed by centrifugation at $300 \mathrm{Xg}$ (1000 rpm) for $5 \mathrm{~min}$ at room temperature.

Passaging cells involved decanting of the culture media followed by rinsing with sterile phosphate buffered saline (PBS). To dissociate the cells, an appropriate volume of pre-warmed trypsin-ethylene diamine tetra acetic acid (EDTA) (Invitrogen) (final concentration of $0.05-0.02 \%$ respectively) was added to a volume sufficient to cover the surface of the culture flasks. Flasks were then returned to the incubator for 2-5 min to allow for cell detachment. Media containing 5\% FCS was then added at three times the volume of the trypsin, to quench the trypsin activity. The collected cells were then transferred to a $15 \mathrm{ml}$ polypropylene centrifuge tube and centrifuged at $300 \mathrm{X}$ $\mathrm{g}(1000 \mathrm{rpm})$ for $5 \mathrm{~min}$ at room temperature. The supernatant was discarded and the remaining cell pellet resuspended in fresh cell culture media and equally divided into new culture dishes. 
Following trypsinisation the percentage of live cells was determined by the use of trypan blue (Invitrogen) exclusion using a haemocytometer. Briefly $10 \mu \mathrm{L}$ of the resuspended cell mixture was diluted in $10 \mu \mathrm{L}$ volume of $0.02 \%$ trypan blue in PBS and $10 \mu \mathrm{L}$ of this mixture was then loaded onto the haemocytometer, and the number of cells which excluded the trypan blue were counted and the appropriate dilution functions were used.

\subsection{Infantile Haemangioma Tissue Culture}

\subsubsection{Tissue Culture Media}

MCDB 131 powder medium (Sigma-Aldrich) containing trace elements and Lglutamine was reconstituted with $1.18 \mathrm{~g} / \mathrm{L}$ sodium bicarbonate (Sigma-Aldrich) to a final volume of $1 \mathrm{~L}$ with $\mathrm{dH}_{2} \mathrm{O}$. To this reconstituted MCDB 131 media, $2 \%$ Penstrep and $2.5 \mu \mathrm{g} / \mathrm{ml}$ Amphotericin B (Sigma-Aldrich) were added prior to filter sterilisation through a $0.2 \mu \mathrm{M}$ filter (Greiner Bio-One). The media was then either used immediately or stored at $4^{\circ} \mathrm{C}$ for up to 8 weeks.

\subsubsection{Infantile Haemangioma Tissue Specimen Preparation}

All surgically excised IH specimens were initially cleansed of blood clots using sterile PBS. They were then all cut into roughly 2-mm explant pieces and submerged in disinfectant media, consisting of MCDB 131 containing $1.18 \mathrm{~g} / \mathrm{L}$ sodium bicarbonate, $10 \%$ Penstrep, $25 \mu \mathrm{g} / \mathrm{ml}$ Amphotericin B and $2 \mathrm{mM} \mathrm{L-}$ 
glutamine (Gibco-BRL), on a rocking platform at room temperature for at least 2 hrs.

\subsubsection{Infantile Haemangioma Explant In Vitro Culture}

Matrigel (Becton-Dickson) was thawed overnight at $4^{\circ} \mathrm{C}$ prior to use. A total of $350 \mu \mathrm{L}$ of the matrigel was aliquoted into each chamber of a 24 well tissue culture plate (Greiner Bio-One) and plates were then left at room temperature, within the laminar flow hoods, to allow for gel formation. A piece of disinfected IH tissue was placed and allowed to settle in the newly formed gel for a further 20 mins. Following setting of the gel around the $\mathrm{IH}$ tissue piece, $1.5 \mathrm{~mL}$ of the sterile filtered MCDB 131 medium containing 1\% FCS was added onto each well. All plates were incubated at $37^{\circ} \mathrm{C}$ in $3 \% \mathrm{CO}_{2} / 97 \%$ air in a Heracell incubator (Kendo Laboratory Products).

\subsubsection{Isolation of Infantile Haemangioma Explant Derived Cells}

After 3 weeks in culture with media changed every 3 days, cells emanating from the $\mathrm{IH}$ explants were released from the Matrigel by treatment with 50 caseinolytic units/well of dispase (Becton-Dickson) for $2 \mathrm{hrs}$ at room temperature within the laminar flow hoods. The liquefied mixture was then collected and placed into a $15 \mathrm{~mL}$ polypropylene centrifuge tube and the dispase activity quenched by diluting in a 10-fold excess of MCDB 131 media containing 5\% FCS. Cells were harvested by centrifuging at $300 \times \mathrm{g}(1000$ rpm) for 5 min at room temperature. 


\subsubsection{Angiotensin II and Blast Proliferation}

Cells harvested from the haemangioma explant cultures were subsequently plated out in 96-well tissue culture plates (Raylab, Auckland, NZ) at a density of $1 \times 10^{5}$ cells per well. Cells were grown in MCDB 131 media, lacking FCS, but containing angiotensin II (Sigma-Aldrich) at concentrations of $0,25,100$ and $150 \mu \mathrm{g} / \mathrm{ml}$ for a total of 6 days, with the media changed at day 3 .

\subsubsection{Image Analysis}

Images of all cell culture experiments were obtained using an Olympus IX51 inverted microscope (upgraded to IX71) fitted with phase contrast objectives and a colour view 1 camera (Tokyo, Japan)

\subsection{Immuno-staining}

\subsubsection{Immunohistochemistry Slide Preparation}

Slides for immunohistochemistry were initially cleaned with $70 \%$ ethanol, dried and then submerged in a freshly prepared solution of $2 \% 3$-aminopropyltriethoxysilane (APES, Sigma) prepared in acetone for $1 \mathrm{~min}$. Slides were then washed in water to remove excess APES and left to dry overnight in an oven at $37^{\circ} \mathrm{C}$. 


\subsubsection{Paraffin Tissue Sections}

$5 \mu \mathrm{M}$ sections of formalin fixed paraffin embedded surgically excised $\mathrm{IH}$ tissues were cut using a Reichert-Jung Biocut microtome (Leica Instruments, Nussloch, Germany). Sections were floated in a water bath and subsequently transferred onto the APES-coated glass slides prior to drying overnight at room temperature.

De-paraffinisation and rehydration of all slides prior to $\mathrm{IHC}$ staining was performed by an initial xylene stage ( 2 changes, 5 min each) followed by a graded ethanol series $(100 \%, 90 \%, 80 \%$ and $70 \%)$ followed by a final rinsing stage in $\mathrm{dH}_{2} \mathrm{O}$ for 5 min.

\subsubsection{Immunocytochemistry Slide Preparation}

Cell cultures were collected from culture plates and were left to adhere onto 8chamber microscope slides (Becton Dickson) in $100 \mu \mathrm{L}$ of cell culture media/chamber overnight prior to fixation with a total volume of $200 \mu \mathrm{L}$ of ice cold acetone/methanol (50:50) for 30 secs in preparation for immediate immunocytochemistry.

The collection of the non-adherent blast structures involved decanting of the cell media from the culture plates followed by centrifuging at $300 \mathrm{Xg}$ (1000 rpm) for $5 \mathrm{~min}$ at room temperature and removal of the supernatant to allow for a final volume of $200 \mu \mathrm{L}$ per slide before cytospinning using the Cytospin 3 (Thermo Shanden, Cheshire, UK). Slides were dried out on the 
bench for 30 mins and fixed as mentioned above in acetone/methanol in preparation for immediate immunocytochemistry.

\subsubsection{Staining Procedures}

\subsubsection{Histochemistry}

Histochemical analysis of all paraffin sections were performed using Mayer's haematoxylin and eosin (H\&E). Slides underwent initial de-paraffinisation and rehydration, as mentioned previously, followed by dehydration in $90 \%$ and $100 \%$ ethanol respectively ( 2 changes each for 5 mins) and were then cleared in xylene ( 2 changes, 3 mins each) followed by mounting in D.P.X (BDH Chemicals Ltd, Poole, UK).

\subsubsection{Fluorescent Immunohistochemistry}

Following routine deparaffinisation and rehydration slides were rinsed in tris buffered saline (TBS, made up of $2.42 \mathrm{~g} / \mathrm{L}$ Tris (Sigma-Aldrich) and $8 \mathrm{~g} / \mathrm{L} \mathrm{NaCL}$ (Sigma-Aldrich), pH 7.5) containing 0.1\% Tween-20 (BDH Chemicals) (TBST) for $5 \mathrm{~min}$. Antigen retrieval was then performed by immersing the slides in coplin jars containing $10 \mathrm{mM}$ sodium citrate $(\mathrm{pH} 6)$ equilibrated in boiling water for $10 \mathrm{~min}$. The coplin jars containing the slides were then allowed to cool to room temperature for 40 mins on the bench. Slides were then rinsed in TBST (3 changes, 5 mins each) followed by quenching of auto-fluorescence by washing the slides in 3 changes of $0.5 \%(w / v)$ sodium borohydride (Sigma- 
Aldrich) freshly prepared in TBS for 15 mins each. Slides were then washed in TBST and blocked using $5 \%$ bovine serum albumin (BSA, Sigma-Aldrich) prepared in TBST for 60 mins at room temperature. This was then followed by overnight incubation at $4^{\circ} \mathrm{C}$ with the appropriate primary antibody diluted in TBST, containing 1\% BSA, accordingly as indicated in Table 2.3.4.4. Slides were then rinsed in TBST ( 3 changes, 5 mins each) and bound antibodies detected using secondary antibody amplification. The appropriate secondary antibody (Goat anti- Mouse alexa 488 (Invitrogen) or Chicken anti-Rabbit Alexa 594 (Invitrogen)) was incubated at room temperature for $2 \mathrm{hrs.}$ Alternatively a tertiary cascade in which an initial incubation of $2 \mathrm{hrs}$ with an anti-Rabbit digoxigenin (Roche diagnostics, NZ) was used prior to incubation with anti-digoxigenin-Rhodamine (Chemicon, Sydney, Australia) conjugate. Slides were washed in TBST in between antibody incubations. Cell nuclei and mounting were performed using 4',6'-diamidino-2-phenylidole (DAPI) in Gold Antifade (Invitrogen). All coverslips were rinsed in $70 \%$ ethanol for 5 mins prior to mounting

\subsubsection{Fluorescent Immunocytochemistry}

Following fixation, attached cells were blocked with TBST containing 5\% BSA for 1 hour prior to incubation with primary antibodies in a similar fashion as that for IHC. Antibodies used for immuno-cytochemistry were detected using secondary amplification. Counterstaining and mounting was as for IHC. 


\subsubsection{Image Analysis}

Immunostained slides were viewed and captured using a Leica TCS 4D confocal laser-scanning microscope fitted with a krypton/argon laser (Leica Lasertechnik, Heidelberg, Germany). Images were then appropriately background corrected and overlaid using Adobe Photoshop versions CS2 software.

\subsubsection{Antibody Concentrations}

\begin{tabular}{|c|c|c|c|}
\hline Antibody Marker & Species & Antibody Dilution & Source of Antibod \\
\hline GLUT-1 & Rabbit & 1 in 500 & Abcam \\
\hline Smooth muscle actin & Rabbit & 1 in 400 & Abcam \\
\hline CD34 & Mouse & 1 in 300 & Abcam \\
\hline CD133 & Rabbit & 1 in 300 & Abcam \\
\hline VEGFR-2 & Rabbit & 1 in 400 & Abcam \\
\hline Angiotensin converting enzyme & Mouse & 1 in 200 & Abcam \\
\hline Angiotensin receptor 1 & Mouse & 1 in 200 & Abcam \\
\hline Angiotensin receptor 2 & Rabbit & 1 in 100 & Abcam \\
\hline CD31 & Mouse & 1 in 400 & Abcam \\
\hline CD29 & Rabbit & 1 in 300 & Abcam \\
\hline Vimentin & Mouse & 1 in 400 & Abcam \\
\hline Sox-9 & Rabbit & 1 in 300 & Abcam \\
\hline Sox-10 & Rabbit & 1 in 300 & Abcam \\
\hline Nestin & Mouse & 1 in 200 & Abcam \\
\hline p75 & Rabbit & 1 in 200 & Abcam \\
\hline Pref-1 & Rabbit & 1 in 200 & Abcam \\
\hline CD45 & Rabbit & 1 in 300 & Abcam \\
\hline GATA-2 & Rabbit & 1 in 300 & Abcam \\
\hline TAL-1 & Rabbit & 1 in 300 & Abcam \\
\hline Brachyury & Rabbit & 1 in 300 & Abcam \\
\hline Glycophorin A & Mouse & 1 in 200 & Abcam \\
\hline Erythropoietin receptor & Mouse & 1 in 200 & Abcam \\
\hline Haemoglobin $\zeta$ & Rabbit & 1 in 200 & Abcam \\
\hline Nanog & Rabbit & 1 in 300 & Abcam \\
\hline
\end{tabular}




\begin{tabular}{llll} 
SSEA-4 & Mouse & 1 in 300 & Abcam \\
OCT-4 & Rabbit & 1 in 300 & Abcam \\
Human placental lactogen & Rabbit & 1 in 300 & Abcam \\
Human chorionic gonadotrophin & Rabbit & 1 in 300 & Abcam \\
Cytokeratin 7 & Rabbit & 1 in 300 & Abcam \\
Anti-mouse Alexa 488 & Goat & 1 in 500 & Invitrogen \\
Anti-rabbit Alexa 594 & Chicken & 1 in 500 & Invitrogen \\
\hline
\end{tabular}




\section{Chapter 3: Primitive Mesodermal Cells with A Neural Crest Stem Cell Phenotype In Proliferating Infantile Haemangioma}

\subsection{Introduction and Overview}

The observation of the segmental distribution in a subgroup of $\mathrm{IH}$ especially those associated with midline structural anomalies that constitute PHACES syndrome ${ }^{13}$ has led us to investigate the involvement of neural crest cells in the aetiology of this condition. Neural crest cells arise from the neural tube and migrate segmentally in the embryo giving rise to the peripheral nervous system, mesenchymal lineages and melanocytes ${ }^{45,64}$. Neural crest stem cells are characterised by the expression of the neurotrophin receptor $(p 75)^{45}$ and the transcription factors, Sox $9 \& 10^{43,65,66}$. This chapter demonstrates the abundant expression of these neural crest stem cell associated proteins, p75, Sox 9 and Sox 10, on the endothelium of proliferating $\mathrm{IH}$. We have previously shown that the cells in the endothelium also express a mesenchymal signature in that they also express the MSC markers, CD29 and vimentin, as well as the haematopoietic associated markers, CD34 and CD133 ${ }^{67,68}$. It is intriguing that this unique expression pattern is also seen in cells of the primitive mesoderm. Cells of the primitive mesoderm additionally express the transcription factor, brachyury ${ }^{69}$.

In this chapter, IH was stained immunohistochemically for proteins associated with the primitive mesoderm. Data is presented to demonstrate that the cells of the endothelium stain for markers of the primitive mesoderm and have a neural crest stem cell phenotype. 
Additionally, the cells from the outgrowths that emanate from IH explants $^{70}$, maintain a similar expression profile, suggesting that it is the cells of the endothelial layer that are responsible for the outgrowths seen in the explant model.

Collectively these data support the hypothesis of $\mathrm{IH}$ being derived from primitive mesoderm and that the cells within the lesion have a neural crest stem cell phenotype and express proteins associated with haematopoietic, endothelial, neural crest and mesenchymal lineages. A model is proposed to account for the natural progression of $\mathrm{IH}$ based upon the multi-potent expression profile of the primitive mesoderm and their neural crest stem cell phenotype to form all the cell lineages detected in proliferating and involuting $\mathrm{IH}$.

\subsection{Contribution}

All experiments were performed by myself and the manuscript was written by myself and edited by my supervisors.

\subsection{Manuscript: Primitive Mesodermal Cells With A Neural Crest} Stem Cell Phenotype Predominate Proliferating Infantile

\section{Haemangioma}




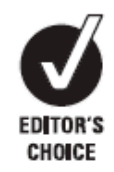

OHCE

\title{
Primitive mesodermal cells with a neural crest stem cell phenotype predominate proliferating infantile haemangioma
}

\author{
Tinte Itinteang, ${ }^{1,2}$ Swee T Tan, ${ }^{1,2,3}$ Helen Brasch, ${ }^{2,4}$ Darren J Day ${ }^{1,2}$
}

'School of Biological Sciences, Victoria University of Wellington, Wellington, New Zealand ${ }^{2}$ Centre for the Study \& Treatment of Vascular Birthmarks, Wellington Regional Plastic, Maxillofacial \& Bums Unit, Hutt Hospital, Wellington, New Zealand

${ }^{3}$ University of Otago,

Wellington, New Zealand

${ }^{4}$ Department of Pathology, Hutt Hospital, Wellington, New Zealand

Correspondence to Professor Swee T Tan Professor Swee T Tan,
Wellington Regional Plastic, Maxillofacial \& Bums Unit, Hutt Hospital, Private Bag 31-907, High Street, Lower Hutt, Wellington, New Zealand: swee.tan@huttvalleydhb.org.nz

Equal senior authors: STT, DJD. This paper was presented, in part, at the Australian and New Zealand Vascular Anomalies Interest Group Meeting, 22 October 2009, Melboume, Australia; the New Zealand Association of Plastic Surgeons' Annual Scientific Meeting, 21 November, 2009, Auckland. New Zealand; the Royal Australasian College of Surgeons' 46th Surgical Research Society Meeting. Adelaide, Australia, 20 November 2009; and the International Society for the Study of Vascular Anomalies Workshop, Brussels, Belgium, 21-24 April 2010.

Accepted 8 June 2010 Published Online First 9 August 2010

\section{ABSTRACT}

Aims Infantile haemangioma is a tumour of the microvasculature characterised by aggressive angiogenesis during infancy and spontaneously gradual involution, often leaving a fibro-fatty residuum. The segmental distribution of a subgroup of infantile haemangioma, especially those associated with midline structural anomalies that constitute posterior fossa malformations-hemangiomas-arterial

anomalies-cardiac defects-eye abnormalities-sternal cleft and supraumbilical raphe syndrome (PHACES), led us to investigate whether neural crest cells might be involved in the aetiology of this tumour.

Methods Immunohistochemical staining on paraffin embedded infantile haemangioma sections and immunocytochemical staining on cells derived from proliferating haemangioma cultures were performed. Results The endothelium of proliferating infantile haemangioma contains abundant cells that express the neurotrophin receptor (p75), a cell surface marker that identifies neural crest cells, and also for brachyury. a transcription factor expressed in cells of the primitive mesoderm. The endothelium is also immunoreactive for the haematopoietic stem cell marker, CD133; the endothelial-haematopoietic stem/progenitor marker, CD34; the endothelial cell markers, CD31 and VEGFR-2; and the mesenchymal stem cell markers, CD29 and vimentin. Additionally, immunoreactivity for the transcription factors, Sox 9 and Sox 10, that are expressed by prospective neural crest cells was also observed. Cells from microvessel-like structures were isolated from in vitro cultured haemangioma tissue explants embedded in a fibrin matrix. Immunostaining of these cells showed that they retained expression of the same lineage-specific markers that are detected on the paraffin embedded tissue sections.

Conclusions These data infer that infantile haemangioma is derived from primitive mesoderm and that the cells within the lesion have a neural crest stem cell phenotype, and they express proteins associated with haematopoietic, endothelial, neural crest and mesenchymal lineages. The authors propose a model to account for the natural progression of infantile haemangioma based upon the multipotent expression profile of the primitive mesoderm and their neural crest stem cell phenotype to form all the cell lineages detected during infantile haemangioma proliferation and involution.

\section{INTRODUCTION}

Infantile haemangioma (figure $1 \mathrm{~A}$ ) is the most common tumour of infancy affecting about $10 \%$ of white children, with a $3: 1$ female:male ratio and a preponderance in premature infants. ${ }^{1-3} \mathrm{It}$ is typified by an initial rapid growth with aggressive angiogenesis during infancy, followed by spontaneous slow involution from 1-5 years of age in which there is diminished cellularity and increasing fibro-fatty deposition. After about 5-10 years of age, involution is complete, and lesions consist predominantly of fibro-fatty tissue with a few mature capillaries. ${ }^{1-4}$

The observation of the segmental distribution of a subgroup of infantile haemangioma, ${ }^{5}$ especially those associated with midline structural anomalies that constitute posterior fossa malformations-hemangiomas-arterial anomalies-cardiac defects-eye abnormalities-sternal cleft and supraumbilical raphe (PHACES) syndrome ${ }^{6}$ (figure 1B), has led us to investigate the role of neural crest cells in the aetiology of infantile haemangioma.

Infantile haemangioma consists of a heterogeneous mixture of cells including immature endothelial cells, ${ }^{7}$ myeloid haematopoietic cells ${ }^{8}$ such as mast cells, ${ }^{9}$ haematopoietic stem cells expressing $\mathrm{CD} 133^{10}$ and mesenchymal stem cells. ${ }^{11}$ Recent reports suggest that the mesenchymal stem cells in haemangioma are recruited from the bone marrow and/or from the adjacent mesenchymal stem cell niches, and that these mesenchymal stem cells terminally differentiate into adipocytes as the lesion involutes. ${ }^{11}$ There is also growing evidence demonstrating that the stem cells in infantile haemangioma are derived from a mesodermal/ mesenchymal phenotype. ${ }^{10}$ The identification of markers associated with endothelial and haematopoietic lineages in these same cells has also been reported. ${ }^{10}$

In this study we used immunofluorescence to investigate the expression of cell surface and cytosolic proteins associated with cells of the neural crest and primitive mesoderm.

\section{MATERIALS AND METHODS}

Immunohistochemistry

Immunohistochemical (IHC) staining was performed on $4 \mu \mathrm{m}$ paraffin-embedded sections of localised infantile haemangioma from 12 patients, aged 3-10 (mean 6) months, according to a protocol approved by the Wellington Regional Ethics Committee.

Routine rehydration followed by antigen retrieval with boiling $10 \mathrm{mM}$ sodium citrate (Sigma-Aldrich, St Louis, Missouri) was performed, and sections were washed in Tris-buffered saline (TBS; $20 \mathrm{mM}$ Tris- $\mathrm{HCl}$ buffer, $\mathrm{pH} 7.6$, containing $135 \mathrm{mM} \mathrm{NaCl}$ ) containing $0.1 \%$ Tween 20 (SigmaAldrich). Autofluorescence was quenched with 

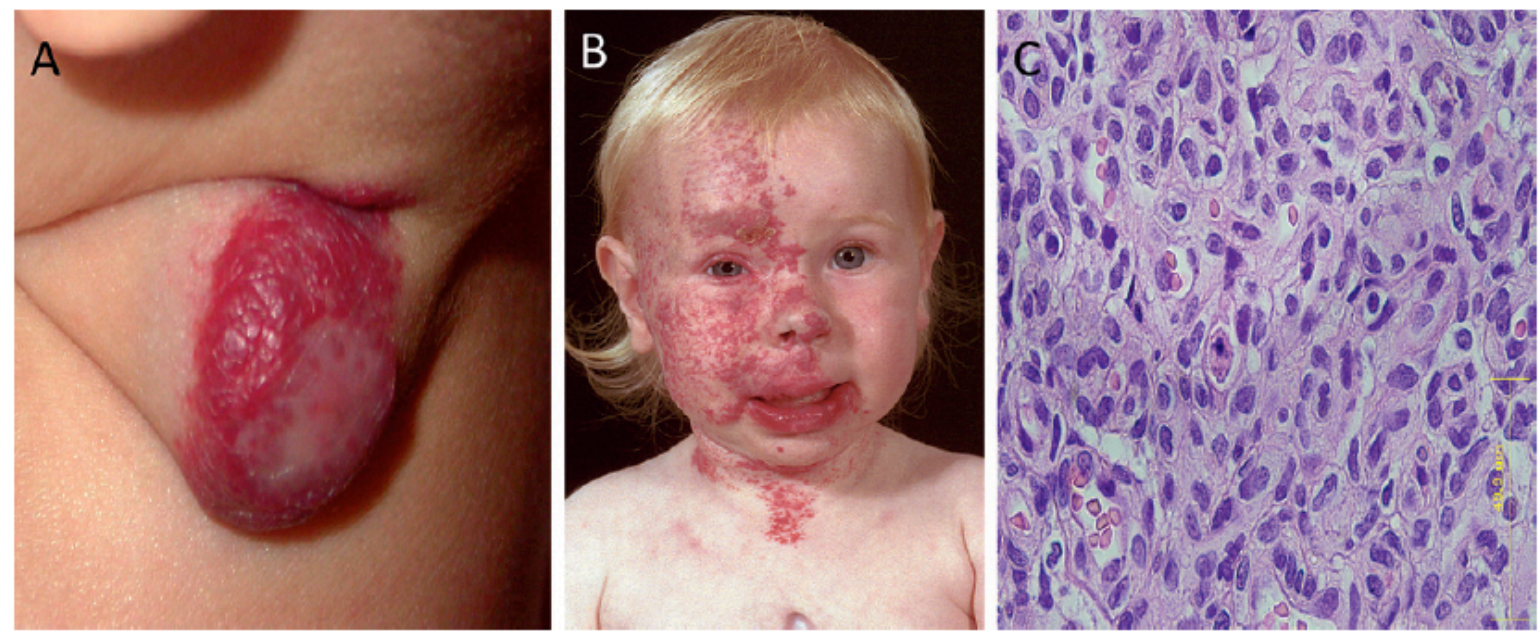

Figure 1 (A) Ulcerated localised proliferating infantile haemangioma on the neck of a 4.5-month-old infant. (B) Girl with segmental facial haemangioma associated with a sternal cleft (posterior fossa malformations-hemangiomas-arterial anomalies-cardiac defects-eye abnormalities-sternal cleft and supraumbilical raphe syndrome (PHACES)). (C) H\&E staining of a proliferating infantile haemangioma showing immature capillaries with tiny lumens lined by plump endothelial cells with an outer concentric pericyte layer.

$0.5 \%$ sodium borohydride (Sigma-Aldrich) prepared in TBS, and the slides then blocked with $5 \%$ bovine serum albumin (SigmaAldrich) prepared in TBS containing $0.1 \%$ Tween 20 for $1 \mathrm{~h}$. Primary antibodies were incubated overnight at $4^{\circ} \mathrm{C}$. Primary antibodies used were CD34, 1:300; smooth muscle actin (SMA), 1:400; VEGFR-2, 1:300; CD133, 1:300; CD31, 1:400; p75, 1:200; Sox-9, 1:300; Sox-10, 1:300; CD29, 1:300; vimentin, 1:400; nestin, 1:200. Primary antibodies were obtained from Abcam (Cambridge, Massachusetts). Bound antibodies were visualised with the appropriate species-specific fluorochrome conjugated secondary antibody (goat antimouse Alexaflor-488 or chicken antirabbit Alexafluor-594 (Invitrogen, Auckland, New Zealand)), with the exception of VEGFR-2, which was detected with an antirabbit digoxigenin (Roche diagnostics, Auckland, New Zealand) conjugate followed by an antidigoxigenin-Rhodamine (Chemicon, Sydney, Australia). All slides were mounted in Antifade Gold (Invitrogen) containing DAPI as counterstain prior to visualisation.

Paraffin sections of uterine fibroid were used as appropriate negative controls to confirm the specificity of staining for $\mathrm{p} 75$, Sox 9 , Sox 10 and brachyury. Control infantile haemangioma samples in which the primary antibodies were omitted showed minimal staining.

\section{In vitro culture of tissue explants}

Surgically resected proliferating infantile haemangioma (figure 1A) biopsies from three patients, aged 3,6 and 8 months, were initially disinfected then cultured in a fibrin gel matrix as previously described by Tan et al ${ }^{12}$ in 24-well plates (Raylab, Auckland, New Zealand). The growth media were changed every 3 days, and following 3 weeks in culture, the cells emanating from the haemangioma explants were harvested from the fibrin gel using 50 caseinolytic units/well of Dispase (Becton-Dickson, Auckland, New Zealand). Dispase activity was quenched with dilution in MCDB (Sigma-Aldrich) media containing 5\% Penstrep (Invitrogen), $0.5 \mu \mathrm{g} / \mathrm{ml}$ Amphotericin $\mathrm{B}$ (Sigma-Aldrich) and $5 \%$ foetal calf serum (Invitrogen) at a dilution of 1:10. Viable cells were then plated and allowed to adhere onto microscope slides divided into eight chambers
(Becton-Dickson) at a density of $1 \times 10^{4}$ cells/chamber prior to fixation with ice-cold acetone/methanol (50:50) for $30 \mathrm{~s}$.

\section{Immunocytochemistry}

Routine immunocytochemistry was performed using CD34, p75, CD29, VEGFR-2 \& CD31. Staining of HeLa cells and the neuroblastoma cell line SY5Y were used as controls.

\section{Microscopy}

Staining was visualised and images captured using a Leica TCS 4D confocal laser-scanning microscope fitted with a krypton/ argon laser (Leica Lasertechnik, Heidelberg, Germany).

\section{RESULTS}

Proliferating infantile haemangima endothelium expressed primitive markers of multiple lineages

Proliferating infantile haemangioma consisted of immature capillaries with tiny lumens lined by plump endothelial cells with an outer concentric pericyte layer (figure 1C). These capillaries displayed an inner endothelium previously characterised by the endothelial marker $\mathrm{CD} 31^{1}$ and the haematopoeticendothelial marker CD $34,{ }^{13}$ the outer pericyte layer confirmed by immunoreactivity (IR) to either smooth muscle actin $(\mathrm{SMA})^{1}{ }^{13}$ or nestin. ${ }^{14}$ Figure 2A shows IR for CD34 (green) in the endothelium and for SMA (red) in the pericyte layer. It is interesting to note that the IR for CD34 is predominantly localised to the luminal side of the endothelium. The endothelium characterised by IR for CD34 (green) in figure $2 \mathrm{~A}, \mathrm{~B}$ also revealed IR for the endothelial cell marker, VEGFR-2 (figure 2B, red) and CD31 (figure 2C, green) as expected. Figure 2C shows that IR for CD133 (red) is localised to the CD31-positive cells. These cells that line the lumen also expressed the mesenchymal stem cell markers; vimentin (figure 2D, green), whereas the pericyte layer was confirmed by IR for SMA (figure 2D, red); and CD29 (figure 2E, red), with the pericyte layer confirmed by IR for nestin (figure 2E, green). The same luminal cells also showed IR for proteins associated with stem cells of primitive origin. The luminal cells demonstrated IR for the neural crest cell 

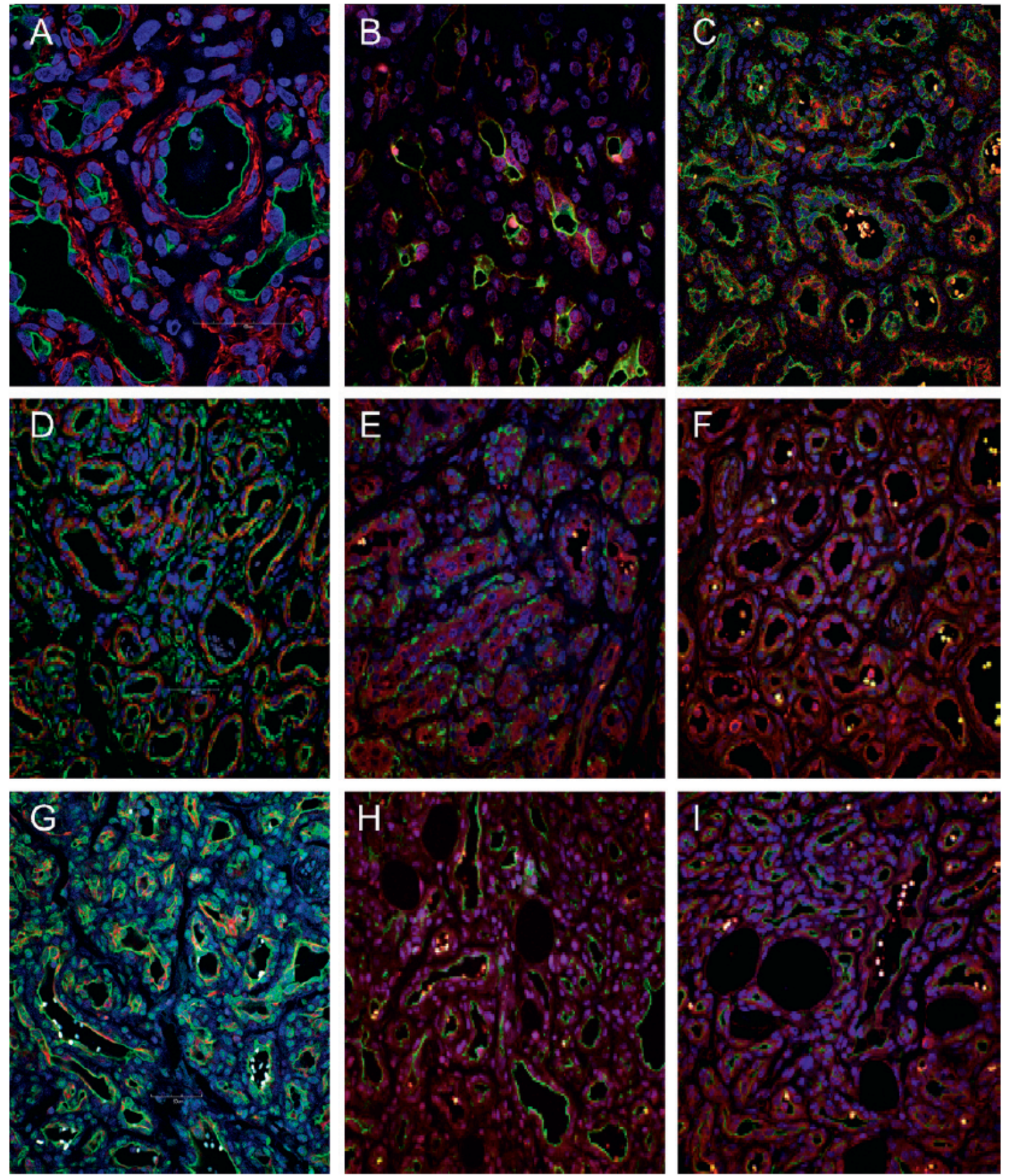

Figure 2 Immunohistochemical staining of representative proliferating haemangioma sections from 12 patients. (A-I) All images are counterstained with DAPI (blue). Staining are as follows: (A) CD34 (green), SMA (red); (B) CD34 (green), VEGFR-2 (red); (C) CD31 (green), CD133 (red); (D) vimentin (green), SMA (red); (E) CD29, (red), nestin (green); (F) p75 (red), nestin (green); (G) CD31 (green), brachyury (red); (H) CD34 (green) Sox 9 (red); (I) CD34 (green), Sox 10 (red). Image 2A was taken at $\times 400$, whereas images (B)-(I) were taken at $\times 200$.

surface marker, p75 (figure 2F, red), distinct from the outer pericyte layer, showing IR to nestin (figure $2 \mathrm{~F}$, green). The endothelium staining positively for $\mathrm{CD} 31$ (figure $2 \mathrm{G}$, green) was also IR to the primitive mesoderm transcription factor, brachyury (figure $2 \mathrm{G}$, red). Consistent with this pattern of staining for primitive markers, the sections also showed IR for 
Original article

the neural crest cell associated transcription factors, Sox 9 (figure $2 \mathrm{H}$, red) and Sox 10 (figure 2I, red). Figure 2, panels $\mathrm{H}$ and I, also show staining for $\mathrm{CD} 34$ (green), identifying the immature endothelial cells lining the capillary lumen.

All infantile haemangioma tissue sections showed IR for Glut1 (data not shown), the IHC marker for infantile haemangioma. ${ }^{2}$ Staining of negative controls, processed and analysed identically, was minimal (data not shown).

\section{Cells emanating from proliferating infantile haemangioma} explants expressed neural crest, endothelial, haemotopoieticendothelial and mesenchymal cell marker

To determine whether the cells forming the microvessels that emanated from proliferating infantile haemangioma explants also expressed markers of multiple lineages, IR for the same array of markers used for IHC staining of tissue sections was evaluated on cells recovered from the fibrin matrix used to culture explanted tissue. Figure $3 \mathrm{~A}$ shows staining for the neural crest cell surface marker, p75 (red), and the endothelial marker, CD31 (green). Doubly labelled cells appear orange in the presented overlayed image. The staining shown in figure $3 \mathrm{~A}$ is in keeping with the p75 staining presented in figure $2 \mathrm{~F}$ and the CD31 staining shown in figure 2C,G. The cells of the microvessels also co-expressed $\mathrm{p} 75$ (red) and CD34 (figure $3 \mathrm{~B}$, green). Staining for CD31 (figures $3 \mathrm{C}, \mathrm{D}$, green) in conjunction with VEGFR-2 (figure 3C, red) and CD29 (figure 3D, red) showed that the cells were positive for all these markers.

Staining of HeLa cells and the SY5Y cells was performed under identical conditions to confirm the specificity of staining. HeLa cells were IR for vimentin, as expected, but showed minimal IR for the other antibodies used in this study (data not shown). SY5Y cells showed IR for p75 (data not shown).

\section{DISCUSSION}

Our IHC data confirm previously documented staining patterns of proliferating infantile haemangioma in that IR for endothelial (VEGFR- $2^{15}$ and $\left.\mathrm{CD} 31^{16}\right)$, haematopoietic-endothelial $\left(\mathrm{CD} 34^{13}\right)$ and the haematopoietic stem cell $\left(\mathrm{CD} 133^{17}\right)$ markers was found. In addition, our IHC data demonstrate the expression of proteins associated with neural crest cells within proliferating infantile haemangioma. IR for neural crest surface marker, $\mathrm{p} 75,{ }^{18}$ also known as neurotrophin receptor, and transcription factors, Sox 9 and Sox 10, is observed in the cells lining the immature capillaries of proliferating lesions. Sox 9 and Sox 10 belong to the group E Sox genes, which are expressed in prospective neural crest cells. ${ }^{19}$ Sox 9 transcription factor has been implicated in neural crest cell induction, ${ }^{19}$ whereas Sox 10 expression has been demonstrated in neural crest stem cells. ${ }^{20} 21$ The expression of Sox 10 transcription factor has been implicated in the maintenance of neural crest stem cell properties. ${ }^{20}$

Taken together, these data suggest a neural crest aetiology for infantile haemangioma. To our knowledge, this is the first report demonstrating the presence of cells expressing p75, Sox 9 and Sox 10 in infantile haemangioma.

The expression pattern of endothelial markers on the immature vessels of proliferating infantile haemangioma has been previously documented and is thought to be due to the angioblastic nature of these immature endothelial cells. ${ }^{7}$ Similar variable expression of endothelial markers has been shown in putative endothelial precursor cells, ${ }^{22}$ embryonic stem cell
Figure 3 Immunocytochemical staining of representative microvesselderived cells from three proliferating haemangioma biopsies. All images are counterstained with DAPI (blue). (A) CD31 (green), p75 (red); (B) CD34 (green), p75 (red); (C) CD31 (green), VEGFR-2 (red); (D) CD31 (green), CD29 (red).
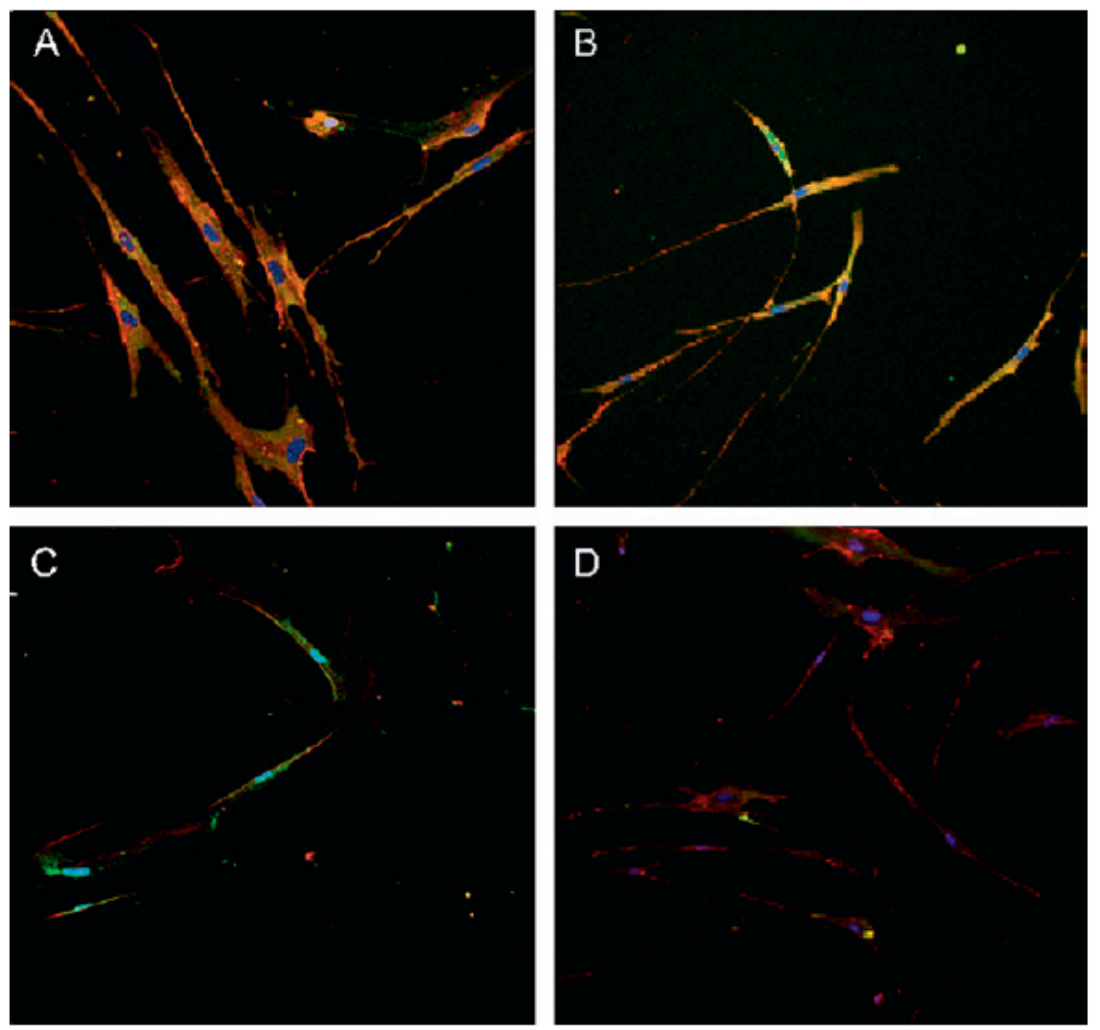
derived endothelial cells ${ }^{23}$ and angioblasts, ${ }^{24} 25$ confirming the primitive nature of these phenotypic endothelial cells.

Within proliferating infantile haemangiomas, we also observed coexpression of CD29 and vimentin on the cells lining the immature vessels. We infer that dual expression of $\mathrm{CD} 29$ and vimentin identifies a mesenchymal stem cell phenotype, consistent with the ability of haemangioma-derived mesenchymal stem cells to differentiate and form adipocytes in vitro, suggesting that the fibro-fatty tissue seen in involuted infantile haemangioma is derived from these mesenchymal stem cells. Other studies have reported that neural crest cells express $\mathrm{CD} 29^{21}$ and vimentin. ${ }^{26}$ Cells staining for mesenchymal markers in proliferating infantile haemangioma have been isolated and shown to differentiate into mesenchymal-derived tissues, such as bone and fat, ${ }^{11}$ as well as neuro-glial cells. ${ }^{10}$ These observations, combined with the demonstration of neural crest stem cell markers on the luminal surface of the immature capillaries that we report, infer that these cells are embryonic stem cell-derived neural crest stem cells with the ability to form cells of both mesenchymal and peripheral neuronal lineages as previously described in the literature. ${ }^{27}$

Expression of CD34, a marker of haematopoietic-endothelial cells, ${ }^{28}$ and CD133, a marker of haematopoietic stem/progenitor cells, ${ }^{29}$ suggests a primitive haematopoietic phenotype. The coexpression of CD133, CD34 and VEGFR-2 is characteristic of stem cells and progenitor cells that have the ability to differentiate into mature endothelial cells. ${ }^{30}{ }^{31}$ Haemangioblasts have been suggested as the common precursor of both haematopoietic and endothelial cells ${ }^{32}$ through a haemogenic endothelium stage ${ }^{33} 34$ and have been proposed to express CD 34 and VEGFR-2..$^{30}{ }^{35}$ We have recently demonstrated the expression of haematopoietic markers on the phenotypic endothelial cells of the immature capillaries of proliferating infantile haemangioma lesions, and we infer that the immature capillaries are structural haemogenic endothelium. ${ }^{36}$ The expression of brachyury by these same cells indicates that they are derived from the primitive mesoderm and have the ability to form haemangioblasts. ${ }^{37}$ Foetal mesenchymal stem cells, on the other hand, are dually positive for vimentin and $\mathrm{CD} 29$ but are negative for both haematopoietic and endothelial markers. ${ }^{38}$ Early embryonic vasculogenesis, however, involves the formation of angioblasts from mesodermal cells, ${ }^{24}$ and the plasticity of mesenchymal cells to differentiate into endothelial cells requires various growth factors, among which, VEGF plays a prominent role. ${ }^{24}{ }^{39}$ These data show that the endothelium of proliferating infantile haemangioma expresses proteins associated with the primitive mesoderm and neural crest stem cells, as well as the previously reported markers of haematopoietic, endothelial and mesenchymal stem cell lineages. It is interesting to speculate that the expression of markers for all four lineages that we report infers that infantile haemangioma has a primitive mesodermal origin with a neural crest stem cell-like phenotype, and that this primitive mesodermal neural crest phenotype has the ability to potentially differentiate down all four lineages, but that is beyond the scope of this paper.

The segmental migratory patterns of neural crest cells in early embryogenesis ${ }^{40}$ may explain the segmental distribution of infantile haemangioma including those occurring in PHACES syndrome ${ }^{6}$. This distribution suggests that infantile haemangioma is a developmental disorder of aberrant neural crest stem cells. These cells we propose have the ability to give rise to both the mature endothelial ${ }^{1}$ and the mature mesenchymal derivatives $^{11}$ observed within involuted infantile haemangioma. We suggest that the fibro-fatty tissue characteristic of involuted infantile haemangioma is due to preferential differentiation of

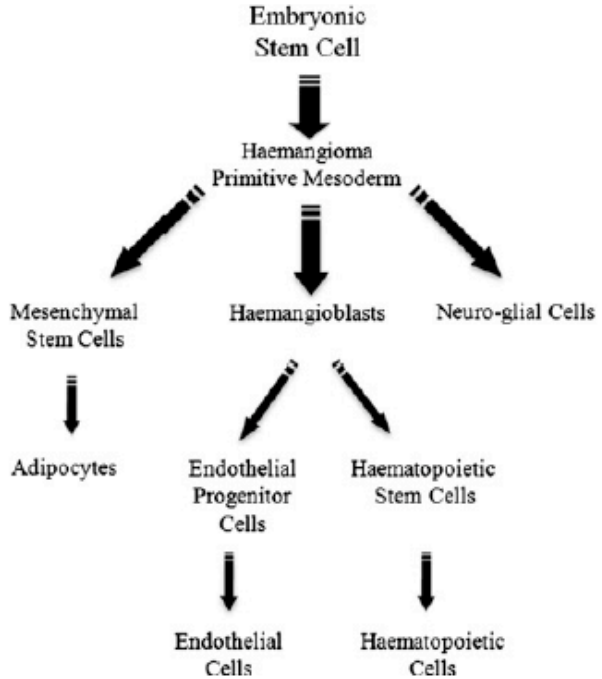

Figure 4 Model illustrating the origin of the cell lineages observed in the progression of infantile haemangioma. Embryonic stem cells are proposed to give rise to the haemangioma primitive mesodermal neural crest stem cells. These cells are able to form mesenchymal stem cells, haemangioblasts and neuro-glial cells. During the proliferative phase of haemangioma, haemangioblasts give rise to the immature endothelial cells that predominate the lesion. Haemangioblasts generate cells of haematopoietic lineage such as mast cells that become highly prominent during the involuting phase of infantile haemangioma. Later in the involuting and involuted phases, the neural crest stem cells give rise mainly to mesenchymal stem cells that are able to differentiate to form the adipocytes that make up the fibro-fatty tissue in the involuted lesion.

these neural crest cells towards the mesenchymal lineage and apoptosis of the endothelial component, possibly in response to the low levels of VEGF ${ }^{1}$ and/or other pro-angiogenic cytokines. Whether these neural crest stem cells give rise to intermediary cells such as haemangioblasts and the mesenchymal stem cells, or whether they have the ability to differentiate directly to form cells of all four lineages is the topic of further investigation.

Figure 4 shows a possible model to account for all the cell types observed in infantile haemangioma that is consistent with, and may be able to account for, the biological characteristics of infantile haemangioma. In this model, we propose that an embryonic stem cell gives rise to infantile haemangiomainitiating primitive mesodermal neural crest cells that proliferate and give rise to haemangioblasts, mesenchymal stem cells and neuro-glial cells. During early tumour growth, haemangioblasts and their progeny predominate, such that the lesion expresses abundant endothelial and haematopoeitic markers. As infantile haemangioma involutes, and levels of VEGF decrease, the endothelial component is reduced, and the mesenchymal component predominates. The mesenchymal stem cells

\section{Take-home message}

Infantile haemangioma endothelium is derived from primitive mesoderm with a neural crest stem cell phenotype, and they co-express proteins associated with haematopoietic, endothelial and mesenchymal lineages. 
Original article

eventually terminally differentiate to form the adipocytes which make up the fibro-fatty deposits within involuted lesions.

Funding We wish to thank the Wellington Regional Plastic Surgery Unit Research \& Education Trust; the Wellington Medical Research Foundation, the Surgical Research Trust; and Pub Charity for their financial support of this project. Tl is supported by a Royal Australasian College of Surgeons' Foundation for Surgery Scholarship.

\section{Competing interests None}

Patient consent Obtained from the parents.

Ethics approval Ethics approval was provided by the Wellington Regional Ethics Committee.

Provenance and peer review Not commissioned; externally peer reviewed.

\section{REFERENCES}

1. Takahashi K, Mulliken JB, Kozakewich HP, et al. Cellular markers that distinguish the phases of hemangioma during infancy and childhood. J Clin invest 1994:93:2357-64

2. North PE, Waner M, Mizeracki A, et al. Glut1: a newly discovered immunohistochemical marker for juvenile hemangiomas. Human Patho 2000;31:11-22.

3. Tan ST, Velickovic M, Ruger BM, et al. Cellular and extracellular markers of hemangioma. Plast Reconstr Surg 2000:106:529-37.

4. Mulliken JB, Glowacki J. Hemangiomas and vascular malformations in infants and children: a classification based on endothelial characteristics. Plast Reconstr Surg 1982;69:412-19

5. Waner $M$, North PE, Scherer KA, et al. The nonrandom distribution of facial hemangiomas. Arch Dermatol 2003:139:869-75.

6. Metry DW, Hawrot A, Attman C, et al. Association of solitary, segmental hemangiomas of the skin with visceral hemangiomatosis. Arch Dermato' 2004:140:591-6.

7. Dosanjh A, Chang J, Bresnick S, et al. In vitro characteristics of neonatal hemangioma endothelial cells: similarities and differences between normal neonatal and fetal endothelial cells. J Cutan Pathol 2000;27:441-50.

8. Ritter MR, Reinisch J, Friedlander SF, et al. Myeloid cells in infantile hemangioma. Am J Pathol 2006;168:621-8.

9. Tan ST, Wallis RA, He Y, et al. Mast cells and hemangioma. Plast Reconstr Surg 2004:113:999-1011.

10. Khan ZA, Boscolo E, Picard A, et al. Multipotential stem cells recapitulate human infantile hemangioma in immunodeficient mice. J Clin lnvest 2008;118:2592-9.

11. Yu Y, Fuhr J, Boye E, et al. Mesenchymal stem cells and adipogenesis in hemangioma irvolution. Stem Cells 2006;24:1605-12.

12. Tan ST, Hasan Q, Velickovic M, et al. A novel in vitro human model of hemangioma. Mod Pathol 2000;13:92-9.

13. Smoller BR, Alfelberg DB. Infantile (juvenile) capillary hemangioma: a tumor of heterogeneous cellular elements. J Cutan Pathol 1993:20:330-6.

14. Ehrmann J, Kolar Z, Mokry J. Nestin as a diagnostic marker: immunohistochemical analysis of its expression in different tumours. $J$ Clin Pathol 2005;58:222-3.

15. Yu Y, Varughese J, Brown LF, et al. Increased Tie2 expression, enhanced response to angiopoietin-1, and dysregulated angiopoie tin-2 expression in hemangioma-derived endothelial cells. Am J Pathol 2001;159:2271-80.
16. Oing L, Ying Y. Joyce B, et al. Differential expression of CD146 in tissues and endothelial cells derived from infantile haemangioma and normal human skin. $J$ Pathol 2003:201:296-302.

17. Boscolo E, Bischoff J. Vasculogenesis in infantile hemangioma. Angiogenesis 2009:12:197-207

18. Morrison SJ, White PM, Zock C, et al. Prospective identification, isolation by flow cytometry, and in vivo self-renewal of multipotent mammalian neural crest stem cells. Cell 1999;96:737-49.

19. Cheung $\mathbf{M}$, Briscoe J. Neural crest development is regulated by the transcription factor Sox9. Development 2003;130:5681-93.

20. $\mathrm{K} \mathrm{m} \mathrm{J}$, Lo L Dormand E, et al. SQX10 maintains multipotency and inhibits neuronal differentiation of neural crest stem cells. Neuron 2003:38:17-31.

21. Iwashita T, Kruger GM, Pardal R, et al. Hirschsprung disease is linked to defects in neural crest stem cell function. Science 2003;301:972-6.

22. Rafii S. Circulating endothelial precursors: mystery, reality, and promise. J Clin invest 2000:105:17-19.

23. McCloskey KE, Smith DA. Jo H, et al. Embryonic stem cell-derived endothelial cells may lack complete functional maturation in vitro. J Vasc Res 2006:43:411-21.

24. Jain RK. Molecular regulation of vessel maturation. Nature Med 2003;9:685-93.

Hatzopoulos AK, Folkman J, Vasile E, et al. Isolation and characterization of endothelial progenitor cells from mouse embryos. Development 1998;125:1457-68.

26. Zller C, Dupin E, Brazeau P, et al. Early segregation of a neuronal precursor cell line in the neural crest as revealed by culture in a chemically defined medium. Cell 1983;32:627-38.

27. Lee G, Kim H, Elkabetz Y, et al. Isolation and directed differentiation of neural crest stem cells derived from human embryonic stem cells. Nature Biotechnol 2007:25:1468-75

28. Krause DS, Fackler $\mathrm{MJ}$, Civin $\mathrm{Cl}$, et al. CD34: structure, biology, and clinical utility. Blood 1996:87:1-13

29. Yin AH, Miraglia S, Zanjani ED, et al. AC133, a novel marker for human hematopoietic stem and progenitor cells. Blood 1997;90:5002-12.

30. Peichev M, Naiyer AJ, Pereira D, et al. Expression of VEGFR-2 and AC133 by circulating human CD34+ cells identifies a population of functional endothelial precursors. Blood 2000;95:952-8.

31. Shantsila E, Watson T, Tse H-F, et al. New insights on endothelial progenitor cell subpopulations and their angiogenic properties. J Am Col Cardiol 2008,51:669-71.

32. Forrai A, Robb L. The hemangioblast between blood and vessels. Cell Cyde 2003:2:86-90.

33. Lancrin C, Sroczynska P. Stephenson C, et al. The haemangioblast generates haematopoietic cells through a haemogenic endothelium stage. Nature 2009:457:892-6.

34. Dieterlen-lièvre $\mathbf{F}$, Jaffredo $T$. Decoding the hemogenic endothelium in mammals. cell. Stem Cell 2009:4:189-90.

35. Zegler BL, Valtieri M, Porada GA, et al. KDR: a key marker defining hematopoietic stem cells. Science 1999;285:1553-7.

36. Itinteang $\mathbf{T}$, Tan ST, Brasch $\mathrm{H}$, et al. Haemogenic endothelium-in infantile haemangioma? J Clin Pathol 2010. In press.

37. Kingsley PD, Malik J, Emerson RL, et al. 'Maturational' globin switching in primary primitive erythroid cells. Blood 2006;107:1665-72.

38. O'Donoghue K, Fisk NM. Fetal stem cells. Best Pract Res Clin Obst Gynaecol 2004:18:853-75.

39. Oswald J, Boxberger S, Jorgensen B, et al. Mesenchymal stem cells can be differentiated into endothelial cells in vitro. Stem Cells 2004:22:377-84.

40. Lee G, Km H. Elkabetz $Y$, et al, Isobtion and directed differentiation of neural crest stem cels derived from human embryonic stem cells. Nature Biotechnology 2007;25:1468-75. 


\section{Chapter 4: Expression of Haemogenic Endothelium Markers In Infantile Haemangioma}

\subsection{Introduction and Overview}

The expression of the haematopoietic markers, CD133 and CD34, and the primitive mesoderm marker, brachyury, as previously described in Chapter 3 of this thesis has led us to investigate the expression of even more primitive haematopoietic markers in proliferating $\mathrm{IH}$.

Endothelial progenitor cells (EPCs) are a population of progenitor cells, characterised by the expression of CD133, CD34 and VEGFR-2, that maintain the ability to differentiate toward both endothelial and haematopoietic lineages ${ }^{35}$. The presence of endothelial progenitor cells in proliferating $\mathrm{IH}$ has previously been demonstrated. Previous reports have highlighted an associated increased level of these cells within the circulation of affected individuals ${ }^{37}$, leading to the notion that $\mathrm{IH}$ arises from these progenitor cells ${ }^{36}$.

In this chapter data is presented to show that it is the same endothelium that expresses these EPC-associated markers, and in an attempt to unravel the link between primitive mesoderm and downstream EPCs we elected to investigate the expression of the intermediate haematopoietic associated markers. Intriguingly it was found that the endothelial layer also expresses markers associated with haemangioblasts, the precursor to EPCs, through the combined expression of angiotensin converting enzyme (ACE), the EPC-markers and primitive mesoderm specific transcription factor, brachyury. Given that haemangioblasts have been shown to generate 
haematopoietic cells through a haemogenic endothelium phenotype, the expression of Tal-1/SCL, a transcription factor associated with the haemogenic endothelium was undertaken. Data is presented to show that Tal1 is expressed on the same endothelium of proliferating $\mathrm{IH}$ demonstrating the novel expression of this phenotype in this lesion.

These findings highlight a novel haemogenic endothelium phenotype and provide a new understanding into the origin of $\mathrm{IH}$. The data also leads to our hypothesis that the increased levels of the downstream EPCs seen in the peripheral circulation of these patients is a consequence of shedding of these haematopoietic EPCs from the haemogenic endothelium phenotype but they are not the cause of these lesions as previously proposed.

\subsection{Contribution}

All experiments and the drafting of the manuscript were carried out by myself and edited appropriately by my supervisors.

\subsection{Manuscript: Haemogenic Endothelium In Infantile}

\section{Haemangioma}




\title{
Haemogenic endothelium in infantile haemangioma
}

\author{
Tinte Itinteang, ${ }^{1,2,3}$ Swee T Tan, ${ }^{1,2,3,4}$ Helen Brasch, ${ }^{2,5}$ Darren J Day ${ }^{1,2,3}$
}

- Additional figures are published online only. To view these files please visit the journal online (http://jcp.bmj. com).

${ }^{1}$ School of Biological Sciences, Victoria University of Wellington, Wellington, New Zealand Wentre for the Study \& ${ }^{2}$ Centre for the Study
Treatment of Vascular Birthmarks, Wellington Region Plastic, Maxillofacial \& Bums Unit, Hutt Hospital, Wellington, New Zealand

${ }^{3}$ Gillies Mclndoe Research Institute, Lower Hutt,

Wellington. New Zealan Wellington, New Zea Wellington New Zago, Wellington, New Zealand SDepartment of Pathology,
Hospital, Wellington, New Hospital,
Zealand Correspondence to
Professor Swee T Tan, Wellington Regional Plastic. Maxillofacial \& Bums Unit, Hut Hospital, Private Bag 31-907, High Street, Lower Hutt, Wellingt, Lower Hut, swee.tan@huttvalleydhb.org.n

Equal senior authors: STT, DJD This paper was presented, in part, at the Royal Australasia College of Surgeons' Annua Scientific Congress, Brisbane, Australia, 11-16 May 2009; the Australian and New Zealand Vascular Anomalies Interest: Group Meeting Melbourne. Australia, 22 October 2009 ; the Australia, 22 October 2009; the New Zealand Association of Plastic Surgeons' Annual Scientific Meeting, Auckland, New Zealand, 21 November 2009; the 18th Internation Society for the Study of Vascular Anomalies Workshop. Brussels, Belgium, 21-24 April, 2010; and the 8th Annual Meeting of the Intemation Society of Stem Cell Research San Francesco, USA. 16-19 San Francesco,
June 2010.

Accepted 26 July 2010 Published Online First 5 October 2010

\section{ABSTRACT}

Background Proliferating infantile haemangioma $(\mathrm{IH})$ is a tumour of the microvasculature composed predominantly of immature endothelial cells. The origin of $\mathrm{IH}$ is unclear, but it has been shown to express markers of both endothelial and haematopoietic lineages, and a role for endothelial progenitor cells in the aetiology of $\mathrm{IH}$ has been suggested. Haemangioblasts are precursors of both endothelial and haematopoietic cells, and their characterisation has identified the expression of cell surface and intracellular proteins that collectively can be used for assigning a haemangioblast phenotype.

Methods The authors used immunohistochemical staining to characterise the expression of primitive haematopoietic-associated proteins in proliferating IHs.

Results and discussion The authors show that the cells forming the capillary endothelium express markers associated with primitive haematopoietic cells.

Additionally, many of these cells express the transcription factors brachyury and GATA-2, indicating a primitive mesodermal origin. They hypothesise that the immature capillaries in $\mathrm{IH}$ are derived from primitive mesodermal cells with haemangioblastic differentiation capabilities. The expression of primitive mesodermal, endothelial and haematopoietic markers by the cells forming the endothelium suggests that the immature capillaries that predominate in proliferating $\mathrm{IH}$ are a haemogenic endothelium phenotype, derived from haemangioblasts.

\section{INTRODUCTION}

Infantile haemangioma $(\mathrm{IH})$, a primary tumour of the microvasculature, is the most common tumour of infancy, affecting about $10 \%$ of white children. It typically undergoes postnatal growth during infancy (proliferating phase) characterised by excessive angiogenesis, followed by spontaneous involution over the next 1-5 years (involuting phase) with continued fibro-fatty deposition that replaces the cellular elements up to 10 years of age (involuted phase). ${ }^{1-3}$ Proliferating $\mathrm{IH}$ is composed predominantly of immature endothelial cells ${ }^{1}$ with a smaller component of myeloid haematopoietic cells ${ }^{45}$ and mesenchymal stem cells. ${ }^{6}$ The lesion consists of immature capillaries with tiny lumens lined by plump endothelial cells with an outer concentric pericyte layer, characterised by its immunoreactivity (IR) for smooth muscle actin (SMA). ${ }^{12}$

Endothelial progenitor cells (EPCs) have been termed haematopoietic stem cells with an innate ability to differentiate into mature endothelial cells.' There is increasing evidence of the presence of an EPC population characterised by the expression of surface antigens CD133, CD34 and vascular endothelial growth factor receptor-2 (VEGFR-2) ${ }^{8}$ within proliferating $\mathrm{IH}$, with a significant increase in this population of cells in the peripheral blood of affected patients. ${ }^{9}$ These EPCs have been presumed to be recruited into proliferating $\mathrm{IH}$ and contribute to the formation of the endothelial component of this tumour. ${ }^{8}$ We have recently shown that the expression of these EPC-associated markers is localised to the endothelium of the immature capillaries in proliferating $\mathrm{IH}^{10}$ and that these same capillaries have a primitive mesoderm neural crest phenotype. ${ }^{11}$

Haemangioblasts are precursors of both haematopoietic and endothelial cells that have been shown to express brachyury, ${ }^{12}$ TAL- $1,{ }^{12}$ VEGFR- $2,{ }^{12}$ GATA-2 ${ }^{13}$ CD $31,{ }^{13} \mathrm{ACE}^{14}$ and CD $34 .{ }^{13}$ The expression of brachyury, ${ }^{15}$ TAL- $1,{ }^{15}$ GATA-2 ${ }^{16}$ and $\mathrm{ACE}^{14}$ on a subset of CD34+/VEGFR-2+ cells has been proposed to delineate primitive precursors from downstream EPCs. Previous reports describing the expression of both haematopoietic and endothelial markers in $\mathrm{IH}$ led us to investigate, in this study, whether cells expressing markers consistent with being haemangioblasts ${ }^{17}$ with the potential to differentiate to cells of both the haematopoietic and endothelial lineages, were present within $\mathrm{IH}$.

\section{METHODS}

Biopsies of proliferating $\mathrm{IH}$ affecting the skin and/ or subcutaneous tissues (figure $1 \mathrm{~A}$ ) from 12 patients, aged 3-10 (mean 6) months, were obtained according to a protocol approved by the Wellington Regional Ethics Committee. The tissues were formalin-fixed and paraffin-embedded using standard procedures. $\mathrm{H} \& \mathrm{E}$ and immunohistochemical (IHC) staining were performed on all $4 \mu \mathrm{m}$ paraffin embedded serial sections. Routine rehydration followed by antigen retrieval with boiling $10 \mathrm{mM}$ sodium citrate (Sigma-Aldrich, St Louis, Missouri) was performed, and sections were washed in Tris-buffered saline (TBS; $20 \mathrm{mM}$ Tris $\mathrm{HCl}$ buffer $\mathrm{pH} 7.6$ containing $135 \mathrm{mM} \mathrm{NaCl}$ ) containing $0.1 \%$ Tween 20 (Sigma-Aldrich). Autofluorescence was quenched with $0.5 \%$ sodium borohydride (Sigma-Aldrich) prepared in TBS, and the slides then blocked with $5 \%$ bovine serum albumin (Sigma-Aldrich) prepared in TBS containing $0.1 \%$ Tween 20 .

Immunolabelling with primary antibodies specific for antihuman brachyury, VEGFR-2, CD34, CD45, ACE, GATA-2, CD133, TAL-1, CD34, CD31, SMA and GLUT-1 (Abcam, Cambridge, Massachusetts) were performed overnight. Bound antibodies were visualised by incubation with the appropriate species-specific fluorochrome-conjugated secondary antibody (goat antimouse Alexaflour 488 or chicken antirabbit Alexaflour 594 (Invitrogen, Auckland, NZ)) for $2 \mathrm{~h}$, with the exception of VEGFR-2 and GATA-2 which were 


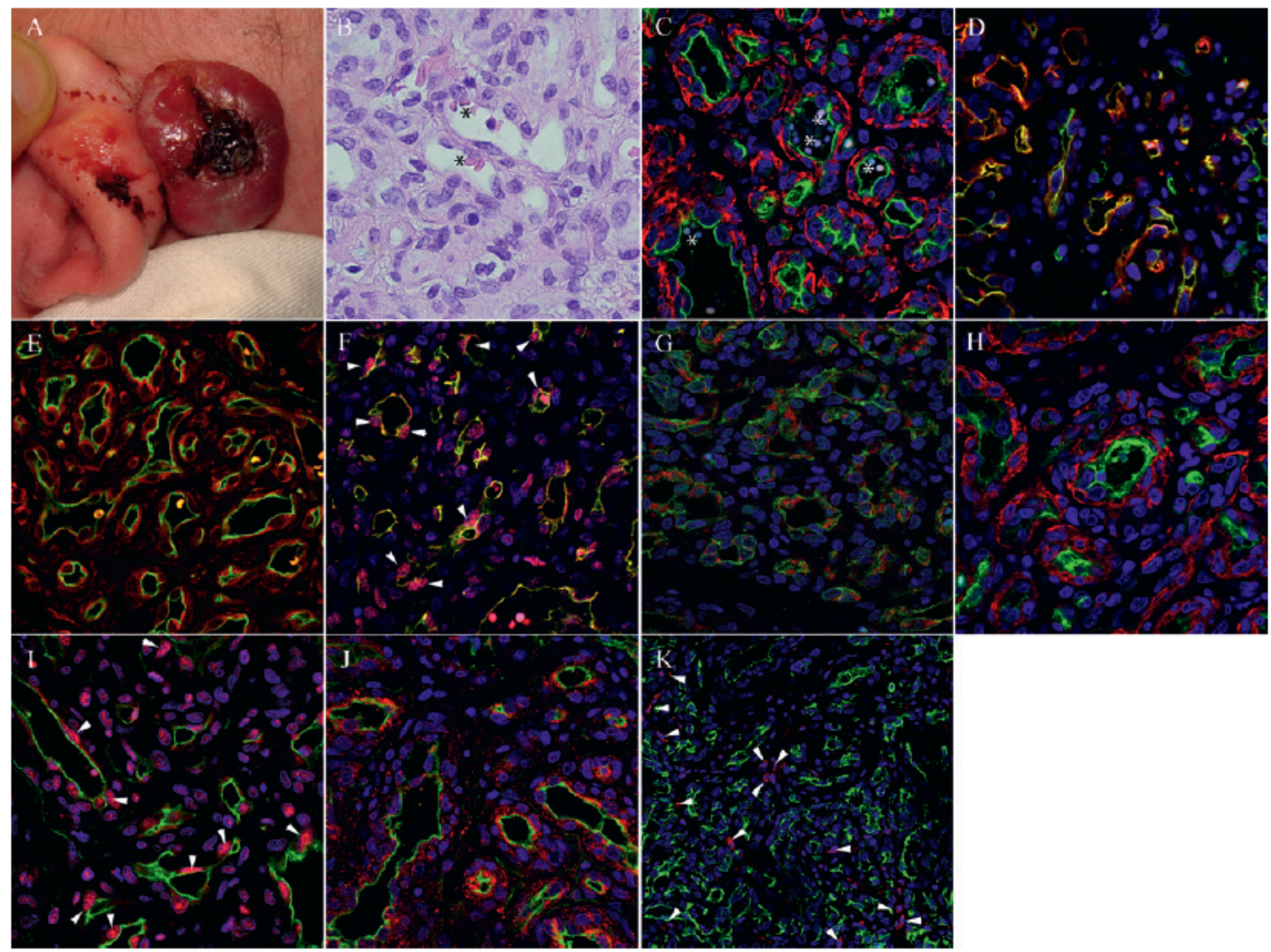

Figure 1 (A) Ulcerated proliferating $\mathrm{IH}$ on the left mastoid region in a 4-month-old boy. (B) H\&E section of this lesion showing immature capillaries with a small lumen lined by plump endothelial cells. Erythrocytes can be seen within the lumen of some, but not all, of the microvessels $\left({ }^{*}\right)$. (C) Endothelium of the immature capillaries, readily identified and distinguished from the pericyte layer by the expression of CD34 (green). The pericyte layer, but not the endothelial cell layer, expresses smooth muscle actin (SMA) (red). (D) Staining for GLUT-1 (red) and CD34 (green). The endothelial cell layer (staining green), but not the pericyte layer, expresses GLUT-1. (E) CD34 ${ }^{+}$cells (green) that form the endothelium are also immunoreactivity (IR) for CD133 (red). (F) CD34+ cells (green) also express vascular endothelial growth factor receptor 2 (red). (G) Endothelial cells forming the endothelium that express CD31 (green) and are also IR for brachyury (red). (H) Staining for SMA (red) identifying the pericyte layer, and ACE (green) which is confined to the endothelium. ACE is expressed only by the cells of the endothelium and not by the pericytes. (I) CD34 ${ }^{+}$cells (green), also express the haematopoietic transcription factor TAL-1 (red). Selected cells that show strong nuclear expression of TAL-1 are identified by white arrowheads. (J) Staining for CD34 (green) and GATA-2 (red). GATA-2 is expressed by the CD $34^{+}$cells. (K) CD31 ${ }^{+}$endothelial cells (green) forming the endothelium do not stain for the mature haematopoietic marker CD45 (red). CD45 IR is restricted to scattered cells within the interstitium (white arrowheads). All images are counterstained with 4',6-diamidino-2-phenylindole (blue).

detected with an antirabbit digoxigenin (Roche diagnostics, Auckland, NZ) conjugate followed by an antidigoxigeninRhodamine (Chemicon, Sydney, Australia) conjugate. Slides were mounted in Antifade Gold (Invitrogen) containing 4,6diamidino-2-phenylindole as counterstain prior to visualisation. Paraffin sections of placental tissue were used as positive controls for CD133, ${ }^{18} \mathrm{CD} 34,{ }^{19} \mathrm{ACE}^{20}$ and GATA-2. ${ }^{21}$ Negative controls included staining of paraffin sections of tissue not expected to be immunoreactive with the same series of antibodies (human uterine fibroid), and also by omission of the primary antibodies when staining selected proliferating $\mathrm{IHs}$ These controls showed the expected staining pattern consistent with the previous reported specificity for the antibodies used. Images were captured using a Leica TCS 4D confocal laserscanning microscope fitted with a krypton/argon laser (Leica Lasertechnik, Heidelberg, Germany).

\section{RESULTS}

Endothelium of proliferating IH expresses endothelial, haematopoietic and primitive mesodermal markers Proliferating $\mathrm{IH}$ tissue taken from all 12 patients in this study showed typical characteristics on $\mathrm{H} \& \mathrm{E}$ sections (figure 1B). Erythrocytes can be seen within the lumen of some $\left({ }^{*}\right)$ but not all microvessels. The microvessels are composed of an inner cell layer that lines the endothelium and an outer concentric pericyte layer. Figure $1 \mathrm{C}$ clearly shows that the cells forming the endothelium are strongly immunoreactivity (IR) for CD34 (green), whereas the cells that make up the outer pericyte layer shows IR for SMA (red), as expected. The CD34 IR is restricted to the lumen face of the cells forming the endothelium as has been shown in other studies. ${ }^{22}$ Autofluorescent erythrocytes can also be seen in some vessels $\left({ }^{*}\right)$. GLUT-1 is an IHC marker used to distinguish IH from other vascular anomalies. ${ }^{3}$ All samples 


\section{Original article}

analysed showed IR for GLUT-1 (figure 1D, red) on the cells lining the endothelium. The GLUT-1 IR is predominantly localised to the lumen face of the cells forming the vessels. Costaining with CD34 (figure 1D, green) generated an orange colour where IR for both CD34 and GLUT-1 occurred. Supplementary figure 1D1-D3 shows the separated and overlayed CD34 and GLUT-1 images presented in figure 1D.

To identify EPCs within the IH lesions, IHC staining for VEGFR-2, CD34 and CD133 was undertaken, as this set of markers has been shown previously to identify EPCs within the circulation. ${ }^{78}$ All proliferating IH sections showed strong IR for VEGFR-2, CD34 and CD133. Figure 1E, F shows representative immunostaining for these markers. Figure 1E shows IR for CD34 (green) to identify the endothelial cells that also expressed the haematopoietic stem cell marker, CD133 (figure 1E, red). IR for CD133 is predominantly expressed on the CD34 cells, with a few cells in the interstitium also staining for CD133. Notably, the cells of the pericyte layer do not express CD133. Supplementary figure 1E1-E3 shows the $\mathrm{CD} 133, \mathrm{CD} 34$ and merged images in separate panels to show more clearly that $\mathrm{CD} 133$ and CD34 are expressed by the same cells that form the endothelium. Figure 1F shows IHC co-staining? for VEGFR-2 (red) and $\mathrm{CD} 34$ (green). Expression of $\mathrm{CD} 34$ is restricted to the plasma membrane on the luminal side of cells lining the vessels, as also shown in figure $1 \mathrm{C}-\mathrm{E}$. Figure $1 \mathrm{~F}$ shows that VEGFR-2 is expressed by the CD34 cells that line the microvessel lumen. Supplementary figure 1F1-F3 presents the CD34 and VEGFR-2 staining in separate panels and shows that VEGFR-2 is expressed in both the cytoplasm and nucleus of the CD34 cells. The white arrowheads in the merged images presented in figure 1F identify cells that strongly express VEGFR-2 and show that these cells are also $\mathrm{CD} 34^{+}$. Collectively, the staining presented in figure $1 \mathrm{E}, \mathrm{F}$ shows that the endothelium expresses markers consistent with an EPC phenotype.

As EPCs are derived from haemangioblasts, ${ }^{23}$ we sought to investigate whether the endothelium was also immunoreactive for the primitive mesodermal marker brachyury, ${ }^{12}$ and for ACE, also called CD $143 .{ }^{14}$ IR for these markers, in conjunction with markers for EPC can be used to identify haemangioblasts. Figure $1 \mathrm{E}, \mathrm{F}$ shows staining of representative $\mathrm{IH}$ sections for brachyury and ACE. All proliferating IH sections analysed showed strong $\mathbb{I R}$ for ACE and brachyury. Figure 1G shows staining for CD31 (green) and brachyury (red). IR for brachyury was restricted to the cells of the endothelium, which also stained for $\mathrm{CD} 31$. Supplementary figure $1 \mathrm{G}$ shows the separate staining for CD31 and brachyury and demonstrates that IR for brachyury is restricted to the $\mathrm{CD} 31^{+}$endothelial cells. Figure $1 \mathrm{H}$ shows staining for ACE (green) and SMA (red). As shown previously, SMA identifies pericytes and does not stain the endothelium (figure 1C). Figure $1 \mathrm{H}$ shows that IR for ACE is localised to the endothelium and is absent from the interstitial cells and pericyte layer. The staining presented in figure $1 \mathrm{G} \& \mathrm{H}$ supports the notion that the expression of haemangioblast associated proteins in the endothelium of $\mathrm{IH}$ as being potentially haemogenic.

It has been previously shown that haemangioblasts generate haematopoietic cells through a haemogenic endothelium stage and that the transcription factor TAL-1 is essential for the establishment of a haemogenic endothelium. ${ }^{17}$ To confirm that the IR demonstrated in figure $1 \mathrm{G}, \mathrm{H}$ identifies a haemogenic endothelium, staining for TAL-1 was undertaken and is presented in figure 1I. All proliferating IHs show strong IR for TAL-1 (red) on the CD34 $4^{+}$(green) cells that form the endothelium (figure 1I). Both nuclear and cytoplasmic staining of
TAL-1 was observed, with the cells forming the endothelium showing the greatest IR for TAL-1 (white arrowheads). To further support the presence of a primitive haemogenic endothelium, staining for GATA-2, a transcription factor expressed early in haematopoietic development, was undertaken. As for TAL-1, all proliferating IHs showed strong IR for GATA-2. Staining of a representative sample is presented in figure 1J. IR for GATA-2 (red) was seen in both the nucleus and cytoplasm of the $\mathrm{CD} 34^{+}$(green) cells forming the endothelium. Supplementary figure $1 \mathrm{~J} 1-\mathrm{J} 3$ shows the separate staining for GATA-2 and $\mathrm{CD} 34$ that more clearly demonstrate the cytoplasmic and nuclear staining seen for GATA-2. Figure 1IJ shows that the transcription factors TAL-1 and GATA-2, which have been previously shown to identify primitive haematopoietic cells, are expressed by the $\mathrm{CD} 34^{+}$endothelium and are consistent with the data presented in figure $1 \mathrm{G}, \mathrm{H}$. Collectively, the data presented in both figure 1 and supplementary figure 1 show that the microvessels in proliferating $\mathrm{IH}$ express proteins associated with a haemogenic endothelium.

Once we had detected primitive haematopoietic markers in $\mathrm{IH}$, we sought to determine whether the endothelium also expressed more mature haematopoietic markers. Figure $1 \mathrm{~K}$ shows staining of a representative section for the mature haematopoietic cell marker, $\mathrm{CD} 45{ }^{24}$ Figure $1 \mathrm{~K}$ shows a representative section stained for $\mathrm{CD} 31$ (green), and for $\mathrm{CD} 45$ (red). The section shows the characteristic strong IR for CD 1 , as expected. The white arrowheads indicate a selection of the $\mathrm{CD} 45$ cells, all of which are in the interstitial space between the $\mathrm{CD} 31^{+}$microvessels. IR for $\mathrm{CD} 45$, the mature myeloid cell marker, was restricted to interstitial space and was not detected on the endothelium of any of the proliferating $\mathrm{IH}$ s studied.

The specificity of the presented IR was confirmed by staining of placenta, which showed IR for CD34, CD133, ACE and GATA-2 (supplementary figure $2 \mathrm{~A}-\mathrm{D}$ ) while there was no or minimal IR when staining of uterine fibroid tissues was performed as a negative control under identical conditions (supplementary figure 2E, F). Similarly, omission of the primary antibody when staining IH sections showed minimal IR (supplementary figure $2 \mathrm{G}, \mathrm{H}$ ).

\section{DISCUSSION}

Haemangioblast had remained elusive until 1998, when its presence as a common precursor for endothelial and haemopoietic cells was reported ${ }^{25}$ Since its discovery, proteins associated with haemangioblast development have been described ${ }^{26}$ and subsequently used to identify cells with the same, or similar, phenotype based upon the expression of transcription factors and cell surface receptors. The nuclear transcription factor TAL-1 (also known as SCL) has been shown to be essential in specifying the formation of haemangioblasts from the primitive mesoderm, ${ }^{27}$ and is also a key regulator of haematopoiesis. ${ }^{28}$ Similarly, the zinc-finger transcription factor, GATA-2, has been shown to be a tissue-specific transcription factor whose activity is essential for cells with haematopoietic potential. ${ }^{29}$ The nuclear and cytoplasmic localisation of this transcription factor in the cells of the endothelium that we report suggests that, although present, some of this transcription factor is not active and therefore localised outside the nucleus. The brachyury gene, also known as $T$, codes for a transcription factor that is expressed by cells derived from the primitive mesoderm, ${ }^{12}$ the cytoplasmic localisation of this transcription factor has been previously reported in early development. ${ }^{30}$ Haemangioblasts have previously been shown to express VEGFR-2, ACE, CD133, CD34 and CD31. We report that the 
brachyury ${ }^{+}$cells lining the immature capillaries in proliferating IH also demonstrate IR for TAL-1, VEGFR-2, CD133, CD34 and CD31, indicating that the cells lining the capillary lumen are derived from cells with haemangioblast capacity. The collective expression of the aforementioned transcription factors and cell surface markers is restricted to the cells lining the microvessel lumen, supporting the presence of a haemogenic endothelium as seen in blood islands of the developing human placenta ${ }^{31}$ We have also recently shown that cells derived from proliferating IH have the ability to form blast colonies in vitro, ${ }_{10}$ which have been shown to be derived from primitive mesoderm,,$^{32}$ which in haemangioma has a neural crest stem cell phenotype. ${ }^{11}$ Consistent with the primitive origin is the absence of staining for the panhaematopoietic marker CD 45 by the endothelium, ${ }^{24}$ although cells showing IR for $\mathrm{CD} 45$ are present in the interstitium between vessels.

Our results confirm, and add to, previous reports showing positive immunostaining in the immature capillaries of proliferating IH for markers associated with endothelial (VEGFR- $2^{37}$ TIE-2, ${ }^{33}$ Ulex ${ }^{4}$ von Willebrands factor, ${ }^{14} \mathrm{CD} 31,{ }^{14}{ }^{43} 35 \mathrm{CD} 34^{2}{ }^{34}$ ) and haematopoietic $\left(\mathrm{CD} 133^{36}\right.$ and $\left.\mathrm{CD} 34^{2}{ }^{24}\right)$ lineages. Haemangioblasts have been identified and generated from embryonic stem cells, ${ }^{13}$ and have been shown to have the dual ability to form cells of both the endothelial and haematopoietic lineages. These data are consistent with the notion that primitive mesoderm gives rise to haemangioblasts that in turn generate the dense network of immature capillaries lined by plump immature endothelial cells within proliferating IH. To our knowledge, this is the first report demonstrating co-expression of brachyury, TAL-1, ACE and GATA-2 in the cells lining the immature capillaries of proliferating $\mathrm{IH}$. The notion that haematopoietic cells are derived from an endothelial phenotype has formed the basis for the concept of the haemogenic endothelium, ${ }^{37}{ }^{38}$ with the expression of TAL-1 being a crucial transcription factor for both the establishment and development of this haemogenic endothelium from haemangioblasts. ${ }^{17}$ It is interesting to speculate that the capillaries that predominate in proliferating $\mathrm{IH}$ are the structural haemogenic endothelium based on the collective expression of the primitive mesodermal and haematopoietic markers, used in this study, on the endothelium of these capillaries.

EPCs within proliferating $\mathrm{IH}^{8}$ have been suggested to be associated with increased levels of these cells in the peripheral circulation of affected patients. ${ }^{9}$ This has led to the suggestion that patients with IH have an inherent increased pool of EPCs that are then recruited into their $\mathrm{IH}$, resulting in subsequent proliferation of the immature endothelial component of this tumour. ${ }^{8}$ This hypothesis implies that the establishment of $\mathrm{IH}$ lesions from circulating EPCs would require upregulation of primitive markers and dedifferentiation to a more primitive phenotype to form the haemogenic endothelium. The dogma that EPCs are primarily produced in the bone marrow, and recruited for tumour growth, has come under increasing scrutiny. ${ }^{39}$ The expression of haematopoietic markers on the endothelium of proliferating $\mathrm{IH}$ shown in our study is more primitive than the markers expressed by circulating EPCs, ${ }^{89}$ supporting our hypothesis that endothelial progenitors originate from within the lesion. We infer that the more definitive downstream EPCs arise from the haemogenic endothelium and are released to the circulation. The expression of proteins associated with dual endothelial and haematopoietic lineages, and the observation of distinct mature endothelial cells ${ }^{1}$ and myeloid cell $s^{5}$ in involuting $\mathrm{IH}$, have led us to speculate that the dual endothelial and haematopoietic lineages seen during the development of IH may be attributed to a common haemangioblast precursor giving rise

\section{Take-home message}

The expression of primitive mesodermal, endothelial and haematopoietic markers by the cells forming the endothelium of infantile haemangioma suggests that the immature capillaries that predominate in proliferating lesions are a haemogenic endothelium phenotype, derived from haemangioblasts.

to a haemogenic endothelium intermediate. We further propose that the increased levels of circulating EPCs seen in patients with $\mathrm{IH}$ are derived from the haemogenic endothelium de novo and are released into the circulation.

The presence of the haemangioblast-derived haemogenic endothelium in IH suggests that this tumour may be best considered as a disorder of embryonic development, and that $\mathrm{IH}$ is a disorder of primitive mesodermal differentiation and proliferation. Our hypothesis is consistent with other studies reporting the presence of cells expressing proteins associated with embryonic haemangioblast progenitors in haemangioblastoma ${ }^{40}$. Our recent observation that this haemogenic endothelium expresses a primitive mesoderm with a neural crest phenotype ${ }^{10} 11$ may provide a clue for the segmental distribution of a subgroup of $\mathrm{IH}$ including those that constitute PHACES (posterior fossa malformations-haemangiomas-arterial anomalies-cardiac defects-eye abnormalities-sternal cleft and supraumbilical raphe) syndrome. ${ }^{11}$ Given that neural crest cells undergo extensive migration during embryonic development and that its derivatives are ubiquitous at birth, further study may provide insights into the localised form of $\mathrm{IH}$, either single or multiple and/or extracutaneous involvement.

An understanding of the development of $\mathrm{IH}$ may not only provide insights into haemogenic endothelium and downstream human haematopoietic and vascular ontogeny but also provide a novel human model for studying primitive haematopoiesis and embryonic development in general.

Funding We wish to thank the Wellington Regional Plastic Surgery Unit Research \& Education Trust, the Wellington Medical Research Foundation, the Surgical Research Trust and Pub Charity for their financial support of this project. Tl is supported by a Royal Australasian College of Surgeons' Foundation for Surgery Scholarship.

Competing interests None

Ethics approval Ethics approval was provided by the Wellington Regional Ethics Committee.

Provenance and peer review Not commissioned; externally peer reviewed.

\section{REFERENCES}

1. Takahashi K, Mulliken JB, Kozakewich HP, et al. Cellular markers that distinguish Takahashi K, Mulliken JB, Kozakewich HP, et al. Cellular markers that
the phases of hemangioma during infancy and childhood. J Clin livest the phases of heman

2. Smoller BR, Alfelberg DB. Infantile (juvenile) capillary hemangioma: a tumor of

heterogeneous cellular elements. J Cutan Pathol 1993;20:330-6.

3. North PE, Waner M, Mizeracki A, et al. Glut1: A newly discovered immunohistochemical marker for juvenile hemangiomas. Human Pathol 2000;31:11-22.

4. Ritter MR, Reinisch J, Friedlander SF, et al. Myeloid cells in infantile hemangioma Am J Pathol 2006:168:621-8.

5. Tan ST, Wallis RA, He Y, et al. Mast cells and hemangioma. Plast Reconstr Surg 2004:113:999-1011.

6. Yu Y, Fuhr J. Boye E, et al. Mesenchymal stem cells and adipogenesis in hemangioma involution. Stem Cells 2006:24:1605-12.

7. Peichev M, Naiyer AJ. Pereira D, et al. Expression of VEGFR-2 and AC133 by circulating human $\mathrm{CD} 34+$ cells identifies a population of functional endothelial precursors. Blood 2000;95:952-8.

8. Yu Y, Fint AF, Mulliken JB, et al. Endothelial progenitor cells in infantile hemangioma. Blood 2004:103:1373-5. 
9. Kleinman ME, Tepper OM, Capla JM, et al. Increased circulating AC133, CD34 endothelial progenitor cells in children with hemangioma. Lymphat Res Biol 2003;4:301-7.

10. Itinteang $\mathbf{T}$, Brasch HD, Tan ST, et al. Expression of components of the renin-angiotensin system in proilferating infantile haemangioma may account for the Propranolol- induced accelerated imvolution. JPRAS 2010: doi 10.1016/.bjps.2010.08.03

11. Itinteang $\mathbf{T}$, Tan ST, Brasch $\mathrm{H}$, et al. Primitive mesodermal cells with a neural crest stem cells phenotype predominate prolfferating haemangioma. $J$ Clin Pathol 2010;63:771-6. doi: 10.1136/jcp.2010.079368

12. Huber TL, Kouskoff $\mathrm{V}$, Joerg Fehling $\mathrm{H}$, et al. Haemangioblast commitment is initiated in the primitive streak of the mouse embryo. Nature 2004;432:625-30. Choi J-H, Ryu Y.S, Kim K.H, et al. In vitto development of a hemangioblast from a human embryonic stem cell, SNUhES\#3. Life Sci 2009;85:39-45.

14. Jokubaitis VJ, Sinka L, Driessen R, et al. Angiotensin-corverting encyme (CD 143) marks hematopoietic stem cells in human embryonic, fetal, and adult hematopoietic tissues. Blood 2008:111:4055-63.

15. Park C, Ma YD, Choi K. Evidence for the hemangioblast. Exp Hematol 2005;33:965-70. 16. Hatzopoulos AK, Folkman J, Vasile E, et al. Isolation and characterization of Lancrin C, Sroczynska P. Stephenson C, et al. The haemangioblast generates haematopoietic cells through a haemogenic endothelium stage. Nature 2009:457:892-6.

8. Mizrak D, Brittan M, Alison MR. CD133: molecule of the moment. $J$ Pathol 2008;218:3-9.

9. Honig A, Rieger L, Kapp M, et al. Immunohistochemistry in human placenTAL tissu

Ito $M$, takura A Ohno Y

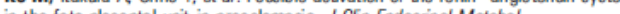
2002:87:1871-8.

21. Chalier JC, Galtier M, Cortez A, et al. Immunocytological evidence for Clacenta 2005-26.282-8.

22. Samulowitz U, Kuhn A, Brachtendorf G, et al. Human endomucin: Distribution pattem, expression on high endothelial venules, and decoration with the MECA-79 pattem, expression on high endothelial veer.

23. Schatteman GC, Awad 0 . Hemangioblasts, angioblasts, and adult endothelial cell progenitors. Anat Rec A Discov Mol Cell Evol Biol 2004;276A:13-21.

24. Dieterlen-lièvre $F$, Jffredo $T$. Decoding the hemogenic endothelium in mammals. Cell Stem Cell 2009:4:189-90.
25. Chai K, Kennedy M, Kazarov A, et al. A common precursor for hematopoietic and endothelial cells. Developoment 1998:125:725-32.

26. Gering M, Rodaway ARF, Gottgens B, et al. The SCL gene specifies haemangioblast development from early mesoderm. EMBO J 1998;17:4029-45.

27. Kallianpur A, Jordan J, Brandt S. The SCLITAL-1 gene is expressed in progenitors of both the hematopoietic and vascular systems during embryogenesis. Blood 1994;83:1200-8.

28. de la Grange PB, Armstrong F, Duval V, et al. Low SCLITAL1 expression reveals its major role in adult hematopoietic myeloid progenitors and stem cells. Blood 2006;108:2998-3004.

29. Minegishi N, Suzuki N, Yokomizo T, et al. Expression and domain-specific function of GATA-2 during differentiation of the hematopoietic precursor cells in midgestation mouse embryos. Blood 2003:102:896-905.

30. Inman KE, Downs KM. Localization of Brachyury (T) in embryonic and extraembryonic tissues during mouse gastrulation. Gene Expression Patterns 2006:6:783-93.

31. Demir R, Seval Y, Huppert女 B. Vasculogenesis and angiogenesis in the early human placenta. Acta Histochemica 2007-109-257-65.

32. Zambidis ET, Park TS, Yu W, et al. Expression of angiotensin-corverting enzyme (CD143) identifies and regulates primitive hemangioblasts derived from human pluripotent stem cells. Blood 2008;112:3601-14.

33. Yu Y, Varughese J, Brown LF, et al. Increased Tie2 expression, enhanced response to Angiopoietin-1, and dysregulated Angiopoietin-2 expression in hemangiomaderived endothelial cells. Am J Pathol 2001;159:2271-80.

34. Martin-Padura I, Castellamau CD. Uccini S, et al. Expression of VE (vascular endotheliall-cadherin and other endothelial-specific markers in of $J$ Pathol 1995;175:51-7.

35. North PE, Waner M, Mizeracki A, et al. A unique microvascular phenotype shared by juvenile hemangiomas and human placenta. Arch Dermatol 2001;137:559-70. Boscolo E, Bischoff J. Vasculogenesis in infantile hemangioma. Angiogenesis 2009;12:197-207.

37. Jaffredo T, Gautier R. Eichmann A et al, Intraaortic hemopoietic cells are derived from endothelial cells during ontogeny. Development 1998;125:4575-83 38. Zovein AC, Hofmann $\mathrm{J}$, Lynch M, et al. Fate tracing reveals the endothel ial origin of

39. Horrevoets AJG. Angiogenic monocytes: another colorful blow to endothelial progenitors. Am J Pathol 2009; 174:1594-6.

40. Glasker $\mathbf{S}$, Li J Xia JB et a! Hemangioblastomas share protein expression with embryonal hemangioblast progenitor cell. Cancer Res 2006;66:4167-72. 


\subsection{Supplementary Figure 1}
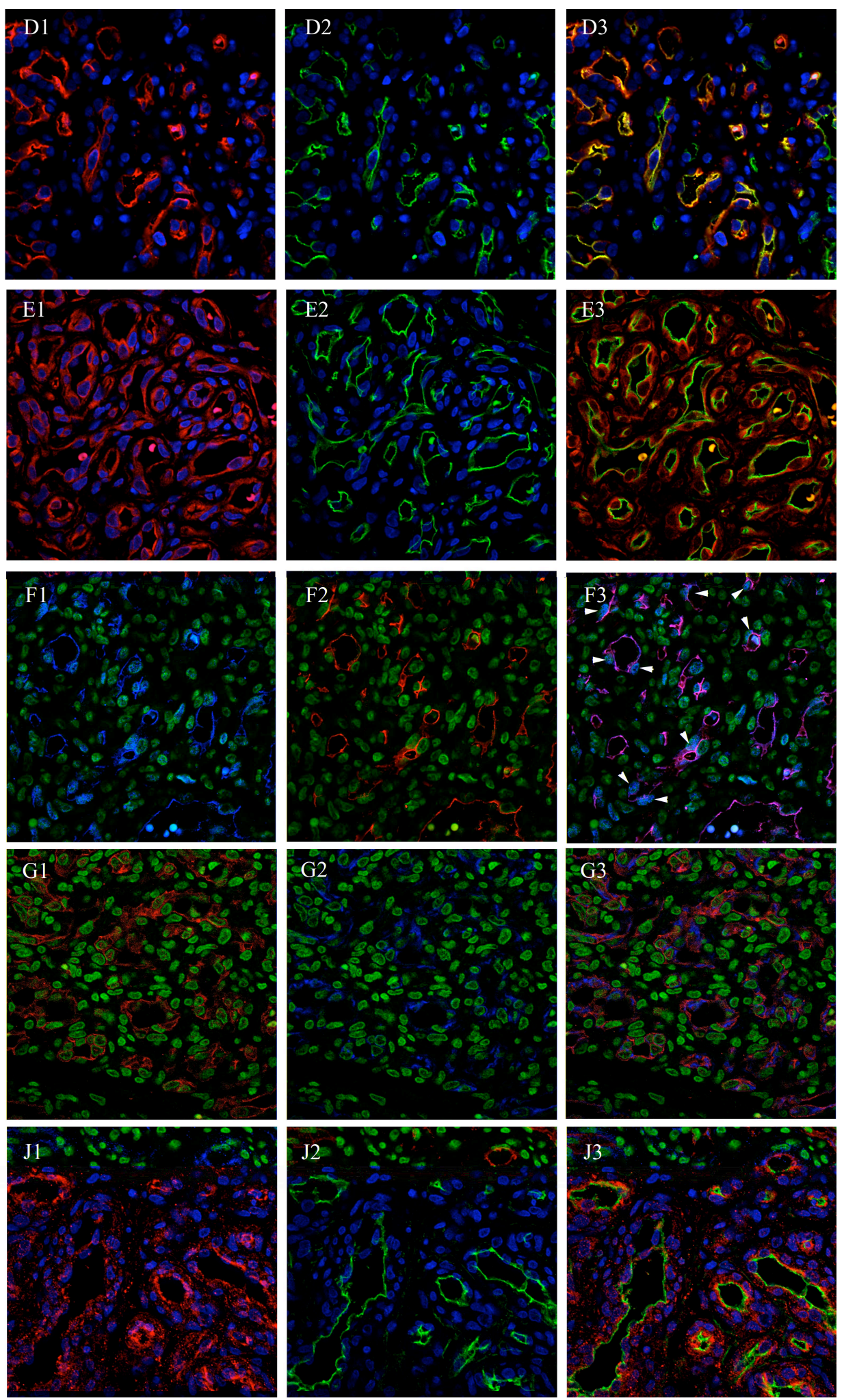

Supplementary Fig 1 
4.5. Supplementary Figure 2

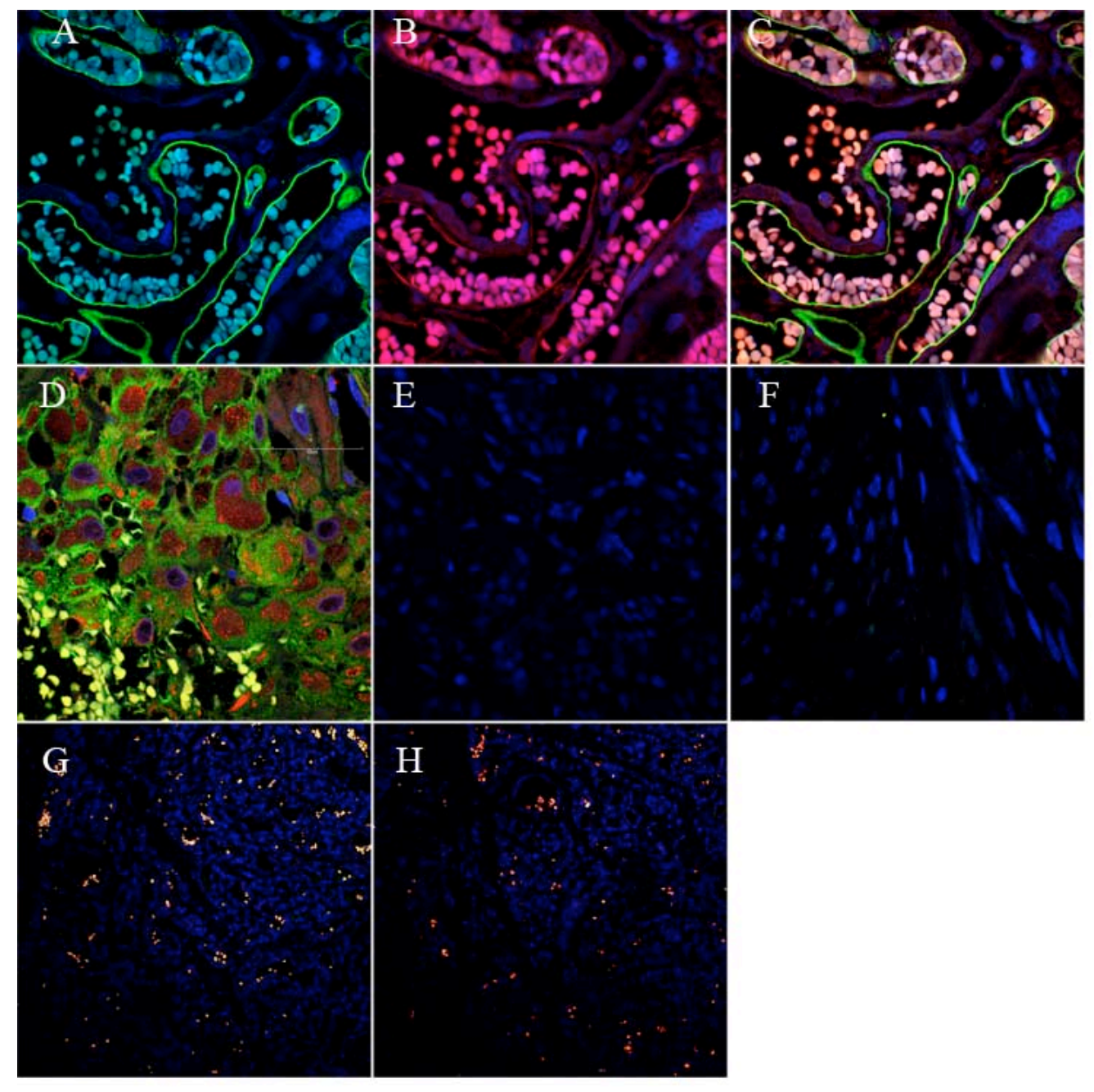

Supplementary Figure 2 


\section{Chapter 5: Primitive Erythropoiesis in Infantile Haemangioma}

\subsection{Introduction and Overview}

The demonstration of a novel haemogenic endothelium phenotype within proliferating $\mathrm{IH}$ as described in Chapter 4, by the expression of primitive haematopoietic associated markers on an endothelium phenotype, suggest the possibility of the functional capacity of $\mathrm{IH}$ to produce downstream haematopoietic cells. In this chapter an investigation into the ability for proliferating $\mathrm{IH}$ to undergo erythropoiesis was undertaken.

Erythropoiesis is the process through which differentiation of multipotent haematopoietic stem cells produces downstream mature enucleated erythrocytes ${ }^{71}$. Functional erythropoiesis is further defined by the ability to demostrate certain stages of erythroid differentiation. In this chapter data is presented to show the in vitro stages of haematopoiesis, in which initial blast-like cells are shown to arise from the cells emanating from proliferating $\mathrm{IH}$ explants that then form blast colonies and eventually give rise to erythrocytes. These are all key stages in erythropoiesis. It is intriguing that we have previously confirmed the expression of the neural crest marker, p75, and the EPC markers, CD133, CD34 and VEGFR-2, on these cells as presented in Chapter 3 of this thesis. This chapter confirms the functional capacity of this primitive phenotype to undergo functional erythropoiesis.

To understand erythropoiesis involves an understanding of the various stages of in utero erythropoiesis. The stages of erythropoiesis are named 
according to the sites of each stage, namely, in chronological order, mesoblastic, hepatic and eventual myeloid ${ }^{49}$. Concomitant with the changes in the embryonic sites of erythropoiesis are the developmental switches in the expression of the various isoforms of haemoglobin $(\mathrm{Hb})$ chain expressed. The initial embryonic $\left(\zeta_{2} \varepsilon_{2}\right) \mathrm{Hb}$ switches to foetal $\left(\alpha_{2} \gamma_{2}\right) \mathrm{Hb}$ at about 6-7 weeks of gestation and is eventually replaced by the adult $\left(\alpha_{2} \beta_{2}\right) \mathrm{Hb}$ postnatally ${ }^{61,62}$.

This chapter reports the expression on the cells of the endothelium in proliferating $\mathrm{IH}$ of the erythropoietin receptor (EPO-R), the receptor for erythropoietin, which is the crucial cytokine required for erythropoiesis as well as the embryonic $\mathrm{Hb} \xi$ protein. The expression of these proteins suggests the potential for proliferating $\mathrm{IH}$ to undergo primitive erythropoiesis. Data is provided to demonstrate that the in vitro cultured IH biopsies are able to undergo erythropoiesis, such that the cells emanating from the explants are able to form erythrocytes in vitro.

\subsection{Contribution}

The concept of primitive erythropoiesis, all in vitro and $\mathrm{IHC}$ experiments were performed by myself. The RT-PCR experiements were performed by $\mathrm{Dr}$ Vishvanath. The manuscript was drafted by myself and edited by my supervisors.

\subsection{Manuscript: Primitive Erythropoiesis In Infantile Haemangioma}




\title{
Primitive erythropoiesis in infantile haemangioma
}

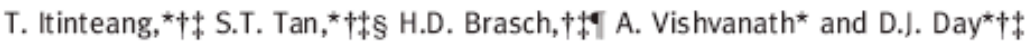

*School of Biobgioal Science, Victoria University of Wellington, Bax 600, Wellington, New Zealand

† Centre for the Study and Treatment of Vescular Birthmarks, Wellington Regional Phstic, Maxilbfacial and Burns Unit, Hutt Hospital, Wellington, New Zonland

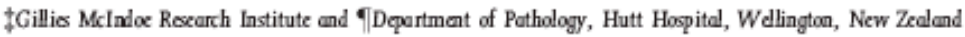

§University of Otogo, Dunedin, New Zooland

\section{Summary}

\section{Correspondence \\ Darrea J. Day. \\ E-mail: Darrem.Day@ww.ac.m. \\ Accepted for publication \\ 6 Dexenter 2010 \\ Funding sources \\ This wak is supportad by grants from the \\ Wellington Regiond Plastic Surgery Unit Roourch \\ \& Edsowtion Trast, the Welliagton Matioul \\ Reourch Fousulation, the Surgioul Rowarch Trast, \\ Pub Charitis and the Caner Sexicty of New \\ Zoubad. A.V. was sepperted, in part, by the \\ Canoer Socicty of New Zedond. T.I. is seppertal \\ by the Boyul Austrelesian College of Surgens' \\ Foualution for Surgery Schdership. \\ Conflicts of interest \\ Nose dedarad. \\ S.T.T. and D.J.D. coutributad to this staly as equal series authors.}

DOI $10.1111 /$ /.1365-2133.2010.10187.
Background Infantile haemangioma (IH) is a tumour of the microvasculature composed predominantly of proliferating endothelial cells. It expresses markers associated with endothelial, haematopoietic and mesenchymal lineages. We have previously shown that the cells forming the capillary endothelium of proliferating IH express cell surface markers and transcriptions factors consistent with it being a haemogenic endothelium.

Objectives We wished to determine whether the expression of transcription factors associated with the erythroid lineage was of physiological relevance and sufficient for $\mathrm{IH}$ tissue cultured in vitro to undergo erythropoiesis.

Methods Immunohistochemical staining of paraffin-embedded sections of proliferating $\mathrm{IH}$ sas undertaken and expression of the embryonically associated haemoglobin $\zeta$ (HBZ) chain and the erythropoietin receptor (EPO-R) was determined. Relative expression of mRNA encoding these proteins was determined by quantitative reverse transcription-polymerase chain reaction using snap-frozen biopsy samples. Differentiation towards erythrocytes was investigated using freshly resected tissue cultured as explants in Matrigel.

Results The endothelium of the microvessels, but not the pericyte layer, was strongly immunoreactive for the EPO-R and the embryonically associated HBZ chain. Abundant expression of transcripts encoding these proteins was also detected, corroborating the immunohistochemical staining. When tissue was grown in culture the cells emanating from $\mathrm{IH}$ explants were able to generate enucleated erythrocytes in vitro. The erythrocytes were immunoreactive for the erythrocyte-specific marker glycophorin A.

Conclusions The microvessels in $\mathrm{IH}$ are a functional haemogenic endothelium that expresses the embryonically associated HBZ chain and is able to form erythrocytes in vitro. IH thus represents a possible extramedullary site for tumour-associated primitive erythropoiesis.
Infantile haemangioma $(\mathrm{IH})$ is a tumour of the microvasculature characterized by excessive angiogenesis during infancy. It affects about $10 \%$ of white infants with predilection for female, white and premature infants. ${ }^{1,2}$ It typically undergoes rapid postnatal growth (proliferative phase) followed by spontaneous involution over a period of up to 10 years, leaving a fibrofatty residuum with a few mature capillaries. ${ }^{1-4}$

The emerging evidence for the role of primitive mesoderm ${ }^{5}$ and its haemogenic endothelium derivative ${ }^{6}$ in proliferating IH has led us to investigate the ability of $\mathrm{IH}$ to form cells of the haematopoietic lineage.

Erythropoiesis is the process through which multipotential haematopoietic stem cells differentiate to form enucleated erythrocytes. ${ }^{7}$ Primitive erythropoiesis originates from primitive mesodermal cells that develop into a haemogenic endothelium, which sequentially give rise to blast colony forming cells, erythroblasts, reticulocytes and definitive enucleated erythrocytes as development progresses. ${ }^{8-10}$ The stages of in utero erythropoiesis have been appropriately termed, in chronological order, mesoblastic, hepatic and myeloid stages corresponding with each subsequent major site of haematopoiesis. ${ }^{11}$ Haemoglobin production also involves three corresponding developmental switches. The initial embryonic ( $\left.\zeta 2 \varepsilon_{2}\right)$ haemoglobin switches to fetal $(\alpha 2 \gamma 2)$ haemoglobin from about 6-7 weeks gestation, and to predominantly adult $(\alpha 2 \beta 2)$ haemoglobin at birth. ${ }^{12}$ 
Here we report that $\mathrm{IH}$ expresses mRNA and protein for erythropoietin receptor (EPO-R) and embryonic haemoglobin $\zeta$ (HBZ), proteins assodated with primitive erythropoiesis, and that cells that emanate from $\mathrm{IH}$ explants ${ }^{4}$ are able to generate erythrocyctes in vitro. IH thus represents a possible extramedullary site for tumour-associated primitive erythropoiesis.

\section{Materials and methods}

\section{Immunolabelling}

Four-micrometre paraffin-embedded sections of $\mathbb{H}$ biopsies were obtained from 12 patients aged 3-10 months (mean 6), according to a protocol approved by the Wellington Regional Ethics Committee. Immunohistochemistry and immunocytochemistry were performed as previously described. ${ }^{5,6,13}$ All primary antibodies were obtained from Abcam (Cambridge, MA, U.S.A.), and were used at the following dilutions: glycophorin A $(1: 200)$, CD34 (1:300), smooth muscle actin (SMA; $1: 400)$, EPO-R $(1: 200)$ and HBZ $(1: 200)$.

Negative controls included staining of paraffin sections of tissue (human uterine fibroid) not expected to be immunoreactive with the same series of antibodies, and also by omission of the primary antibodies when staining proliferating $\mathbb{I H s}$ excised from three patients. These controls showed the expected staining pattern consistent with the previous reported specificity for the antibodies used ${ }^{5,6}$ (data not shown).

\section{Quantitative reverse transcription-polymerase chain reaction}

RNA was extracted from fresh proliferating $\mathbb{I H}$ biopsies of five patients aged 3-8 months (mean 5), and quantitative reverse transcription-polymerase chain reaction ( $q R T-P C R)$ was performed using primers specific for EPO-R and HBZ. Expression relative to that for glyceraldehyde-3-phosphate dehydrogenase (GAPDH) is reported as $\triangle \mathrm{Ct}$ value. Primers for $\mathrm{HBZ}$ and GAPDH have been previously reported. ${ }^{14}$ Primers for EPO-R were: forward CTCATCCTCGTGGTCATCCT, reverse GCTGGAAGTTACCCTTGTGG. Amplification was achieved using an iQ qPCR system (Bio-Rad Laboratories, Hercules, CA, U.S.A.) with an initial denaturation at $95^{\circ} \mathrm{C}$ for $3 \mathrm{~min}$, followed by 35 cycles of denaturation at $95{ }^{\circ} \mathrm{C}$ for $15 \mathrm{~s}$, annealing at $60^{\circ} \mathrm{C}$ for $20 \mathrm{~s}$ and extension at $72{ }^{\circ} \mathrm{C}$ for $20 \mathrm{~s}$. Data were collected during the extension step and melt-curve analysis and agarose gel electrophoresis performed to confirm the specificity of amplification (data not shown).

\section{Cell culture}

Fresh proliferating IH biopsies from five patients aged 3-8 months (mean 5), and two patients with involuted $\mathrm{IH}$, aged 8 and 10 years, were cultured using an in vitro explant model as previously described by Tan et $\mathbf{l}^{4}{ }^{4}$ For all experiments fibrin was replaced with Matrigel (Becton Didkinson, Auckland, New Zealand). Erythrocytes formed in culture were imaged using an Olympus IXS1 inverted microscope (upgraded to IX71) fitted with phase contrast objectives and a Colour View 1 camera (Olympus, Tokyo, Japan). Peripheral blood from each patient was also cultured in parallel as a control. Erythrocytes were collected and processed for immunocytochemistry as previously described. ${ }^{5}$

\section{Results}

The capillary endothelium of haemangioma expresses erythropoietin receptor and haemoglobin $\zeta$

The production of erythrocytes depends on the interaction between EPO-R and its ligand, erythropoietin. ${ }^{15}$ The process of erythropoies is is intricately linked to haemoglobin production and the expression of HBZ is associated with embryonic haemoglobin production during the first trimester of pregnancy. ${ }^{16}$ Figure 1a shows that immature capillaries of proliferating $\mathrm{IH}$ consist of an inner endothelial layer that expresses CD34 (green) and an outer concentric pericyte layer that expresses SMA (red). Figure $1 \mathrm{~b}$ shows that the cells of the capillary endothelium express EPO-R (green), whereas the cells of the outer pericyte layer that express SMA (red) do not. Figure 1c shows that the cells of the endothelium are immunoreactive for both EPO-R (green) and HBZ (red). qRT-PCR of mRNA isolated from tissue confirmed the abundant expression of both $\mathrm{HBZ}$ and EPO-R transcripts relative to that for the control reference gene GAPDH (mean \pm SEM: HBZ $\Delta \mathrm{Ct}=$ $4 \cdot 5 \pm 0.8 ; \mathrm{FPO}-\mathrm{R} \Delta \mathrm{Ct}=10 \cdot 2 \pm 4)$.

\section{Proliferating haemangioma explants generate erythrocytes in vitro}

The functional capacity of proliferating $\mathbb{H}$ to generate erythrocytes was revealed following in vitro growth as explants. On initial culturing of explants there were no cells visible within the Matrigel (Fig. S1a; see Supporting Information) and following 2 weeks in culture outgrowths could be seen in most of the explants of proliferating $\mathbb{H}$ (Fig. S1b; see Supporting Information). Consistent with observations of Tan et d.," no visible outgrowths were seen in the involuted $\mathrm{IH}$ explants (data not shown). We have previously shown that the cells that form the explant outgrowths express haematopoietic-associated markers, ${ }^{5}$ and here we report that following 1 month in culture blast-like cells can be seen arising from these outgrowths (Fig. S1c; see Supporting Information), that then go on to form blast colonies after 3 months in culture (Fig. S1d; see Supporting Information). Following extended periods of culture ( 5 months) biconcave cells resembling erythrocytes were visible within the Matrigel culture (Fig. 1d, e, arrows) of proliferating $\mathrm{IH}$ lesions. The biconcave cells were present only in regions of Matrigel that had previously contained regions of outgrowth. These biconcave cells were confirmed to be erythrocytes by the expression of the erythrocyte-specific marker, glycophorin A (Fig. 1e, bottom, green). Peripheral blood obtained from the same patient 

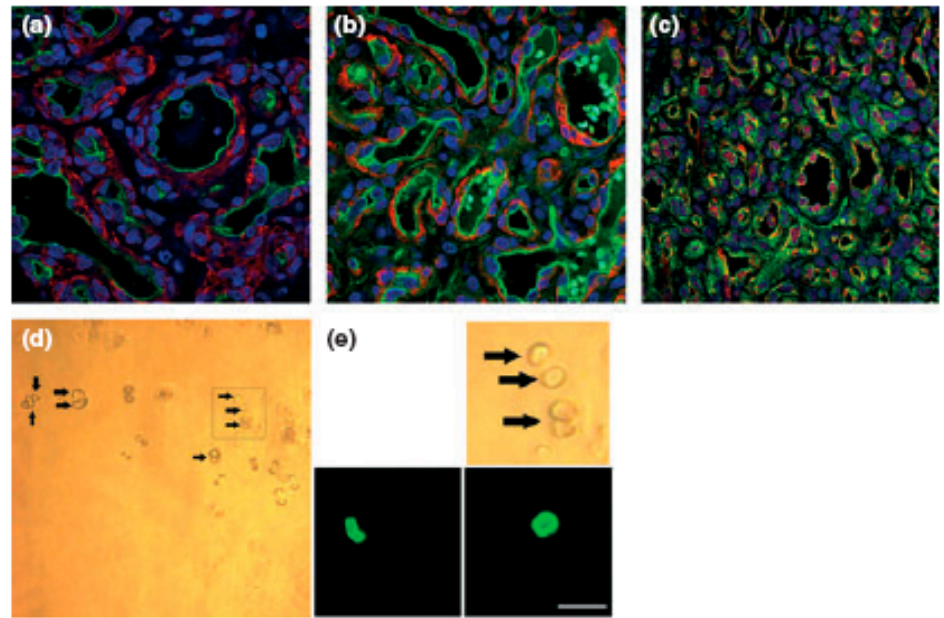

Fig 1. Erythropoiesis in proliferating infantle haemangioma (IH). (2-c) Representative immunohistochemical staining of proliferating IH. (a) The immature capillaries of $\mathrm{IH}$ consist of a CD34+ endothelium (green) surrounded by a concentric layer of pericyte-like cells that are immunoreactive for smooth muscle actin (SMA; red). (b) The endothelium is immunoreactive for erythropoietin receptor (EPO-R; green) but the SMA+ pericyte byer (red) is not. (c) The EPO-R+ cells (green) of the endothelium are also immunoreactive for haemoglobin $\zeta$ (red). (d) After 5 months in culture biconcave cells morphologically simlar to erythrocytes are observed (black arrows). (e) The boxed region containing biconcave celk presented in (d) is shown enlarged; the cells are enucleated and are immunoreactive for the erythrocyte-specific protein glycophorin A (green; bottom). (2-c, e) Coumterstained with 4',6-diamidino-2-phenylindole (blue) to identify cell nudei. Scale ber (e), $10 \mu \mathrm{m}$.

cultured under identical conditions showed no surviving erythrocytes after 3 weeks (data not shown), confirming that erythrocytes that stained positively for glycophorin A were formed $d \varepsilon$ now during culture of proliferating IH lesions rather than originating from carry-over of peripheral blood contaminating the tissue piece.

\section{Discussion}

The capillaries of IH consist of an inner endothelial layer, characterized by the expression of endothelial markers, and an outer concentric layer, characterized by the expression of SMA. ${ }^{5,6.13}$ We have recently shown that this endothelial layer of the capillaries reflects a primitive mesodermal phenotype, with the expression of brachyury, ${ }^{5}$ and with potential haemogenic capacity, through the expression of TAL-1, GATA-2, angiotensin-converting enzyme (ACE), CD133 and CD $34 .^{6}$

The concept of haematopoiesis originating from an endothelial phenotype forms the basis of the concept of the haemogenic endothelium. ${ }^{17-19}$ Here we demonstrate the expression of erythopoietic-associated proteins by the endothelium of the capillaries of proliferating $\mathrm{IH}$. It is intriguing that the expression of EPO-R, a receptor for the ligand erythropoietin, ${ }^{15}$ which is the major haematopoietic associated growth factor responsible for the proliferation, survival and differentiation of erythroid progenitor cells, ${ }^{20}$ is present on the endothelium of proliferating IH. Reports have confirmed the expression of EPO-R in extra-embryonic yolk sac blood islands, ${ }^{20}$ the site of primitive haematopoiesis during the first trimester of pregnancy where HBZ is also expressed. ${ }^{16,21}$
The morphological appearance of blast colony forming units, the precursor to erythroblasts, after 3 months in culture of IH explants, provides further evidence of the presence of another crucial lineage in the overall process of erythropoiesis. We have previously isolated and confirmed the formation of this blast phenotype from $\mathrm{IH}$, by the expression of $\mathrm{CD} 34$, vasallar endothelial growth factor receptor-2, CD133 and ACE, ${ }^{13}$ and here we show the functional ability of cultured $\mathbb{I H}$ explants to form enucleated erythrocytes in vitro. To the best of our knowledge, our report is the first to show that $\mathrm{IH}$ is a potential site of extramedullary erythropoiesis. We have also previously speculated that it is the cells of the endothelial layer in proliferating IH that form the cells in the explant outgrowths. This suggestion is consistent with the expression of primitive mesoderm-associated proteins by the endothelium and the cells of the outgrowths, but not the cells of the pericyte layer. ${ }^{5}$ We have now demonstrated that these cells have the capacity to undergo functional extramedullary erythropoiesis, although the molecular mechanism and pathway involved require further investigation. Similarly, whether the observed extramedullary erythropoiesis observed in culture takes place in viwo within proliferating $\mathbb{I H}$, and if it contributes to the patient's circulating erythrocytes, are yet to be determined.

We infer that the haemogenic endothelium of proliferating IH has the capacity to form cells of the haematopoietic lineage. Given the evidence for formation of erythrocytes and myeloid cells during primitive blood island formation, ${ }^{22}$ it is also exciting to speculate that cells of the myeloid lineage, e.g. mast cells, found in abundance within $\mathrm{IH}^{23}$ may be 
downstream progenitors of the process of primitive haematopoietic differentiation.

This paper contributes to an emerging body of evidence pointing to $\mathrm{IH}$ as a tumour involving the proliferation and eventual differentiation of primitive mesodermal cells. This finding gives new insights into the pathogenesis of $\mathbb{H}$, as a possible extramedullary site of haematopoiesis, and may form the basis for a novel model for investigating the process of human haematopoietic ontogeny.

\section{What's already known about this topic?}

- Cells of the endothelial, haematopoietic and mesenchymal lineages have been identified in infantile haemangioma $(\mathrm{IH})$.

- The capillary endothelium of $\mathbb{H}$ expresses transcription factors associated with haematopoiesis.

\section{What does this study add?}

- We demonstrate that the endothelium of $\mathrm{IH}$ expresses the embryonically associated haemoglobin $\zeta$ chain and the erythropoietin receptor.

- When cultured in vitro explanted IH tissue can be differentiated to form enucleated erythrocytes.

\section{References}

1 Tथn ST, Itinteang T, Leadbitter P. Low-dose propranolol for infantile heemangioma. J Flast Rocwatr Aathet Sarg 201 1; 64:292-9.

2 Takahashi K, Mulliken JB, Kozakewich HP et d. Cellukr markers that distinguish the phases of hemangioma during infancy and childhood. J Clin Irot 1994; 93:2357-64.

3 Frieden IJ, Eichenfield LF, Esterly NB et d. Guidelines of care for hemangiomas of infancy. American Academy of Dermatology Guidelines/Outcomes Committee. J An Acal Dernotel 1997; 37:6317.

4 Tan ST, Hasan $Q$, Velickovic $M$ et al. A novel in vitro human model of hemangioma. Mod Pothe 2000; 13:92-9.

5 Itinteang T, Tan ST, Brasch H, Day DJ. Primitive mesodermal celk with a neural crest stem cell phenotype predominate proliferating infantile heemangioma. J Clia Putbol 2010; 63:771-6.

6 Itinteang T, Tan ST, Brasch H, Day DJ. Haemogenic endothelium in infantile heemangioma. J Ch Pothe 2010; 63:982-6.

7 Richmond TD, Chohथn M, Barber DL. Turning cells red: signal transduction mediated by erythropoietin. Trods Cell Biel 2005; 15:146-55.

8 Dieterlen-Lièvre F, Jaffredo T. Decoding the hemogenic endothelinm in mammals. Cell Sten Cell 2009; 4:189-90.

9 Ema M, Yokomizo T, Wakamatsu A et al. Primitive erythropoiesis from mesodermal precursors expressing VE-cadherin, PECAM-1, The2, endoglin, and CD34 in the mouse embryo. Bbod 2006; 108:4018-24.

10 Gregory C, Eaves A. Three stages of erythropoietic progenitor cell differentiation distinguished by a number of physical and biologic properties. Bbod 1978; 51:527-37.
11 Al-Mufti R, Hambley H, Farzaneh F, Nicolaides K. Fetal and embryonic hemoglobins in erythroblasts from fetal blood and fetal cells enriched from maternal blood in fetal anemia. Haenatbogica $2001 ; 86: 1270-6$.

12 Peschle C, Mavilio F, Carè A et al. Haemoglobin switching in human embryos: asynchrony of zeta-alpha and epsion-gammaglobin switches in primitive and definitive erythropoietic lineage. Natare $1985 ; 313: 235-8$.

13 Itinteang T, Brasch HD, Tan ST, Day DJ. Expression of components of the renin-angiotensin system in proliferating infantile haemangioma may account for the propranobl-induced accelerated involution. J Phast Reowstr Aethet Sarg doi: 10.1016/j.bjps.2010.08.039.

14 Hogh AM, Hvild TVF, Christensen B et al. zeta-, epsibn-, and gamma-Globin mRNA in blood samples and CD71(+) cell fractions from fetuses and from pregnant and nonpregnant women, with special attention to identification of fetal erythroblasts. Oh Chen 2001; 47:645-53.

15 Moritz KM, Lim GB, Wintour EM. Developmental regulation of erythropoietin and erythropoiesis. Am J Physiel Regul Integr Comp Physid 1997; 273 :R1829-44.

16 Schechter AN. Hemoghbin research and the origins of molecular medicine. Bbod 2008; 112:3927-38.

17 Jaffredo T, Gautier R, Eichmann A, Dieterlen-Lièvre F. Intrazortic hemopoietic celk are derived from endothelial cells during ontogeny. Derelqunent 1998; 125:4575-83.

18 Eilken HM, Nishikawa S-I, Schroeder T. Contimuous single-cell imaging of blood generation from heemogenic endothelium. Noture $2009 ; 457: 896-900$.

19 Zovein AC, Hofmann J, Lynch M et al. Fate tracing reveals the endothelial origin of hemetopoietic stem cells. Cell Sten Cell 2008; $3: 625-36$.

20 Kertesz N, Wu J, Chen THP et al. The role of erythropoietin in regulating angiogenesis. Dev Biel 2004; 276:101-10.

21 Qiu C, Olivier EN, Velho M, Bouhassira EE. Globin switches in yolk sac-like primitive and fetal-like definitive red blood cells produced from human embryonic stem cells. Bbod 2008; 111:2400-8.

22 Kanfman DS, Hanson ET, Lewis RL et al. Hematopoietic colonyforming celk derived from human embryonic stem cells. Proc Nad Aood Sei USA 2001; 98:10716-21.

23 Tan ST, Wallis RA, He Y, Davis PF. Mast cells and hemangioma. Plast Rloowstr Sarg 2004; 113:999-1011.

\section{Supporting Information}

Additional Supporting Information may be found in the online version of the article:

Fig S1. (a-d) A representative proliferating infantile haemangioma (IH) explant culture. (a) No cells are visible in the Matrigel at the edge of the $\mathbb{I H}$ explant culture at day 0 . (b) After 2 weeks in culture cellular outgrowths are clearly visible emanating from the IH explant. (c) Following 1 month in culture blast-like cells are visible arising from the cellular outgrowths. (d) After 3 months in culture multiple blast colonies are clearly visible.

Please note: Wiley-Blackwell are not responsible for the content or functionality of any supporting materials supplied by the authors. Any queries (other than missing material) should be directed to the corresponding author for the article. 
5.4. Supplementary Figure 1

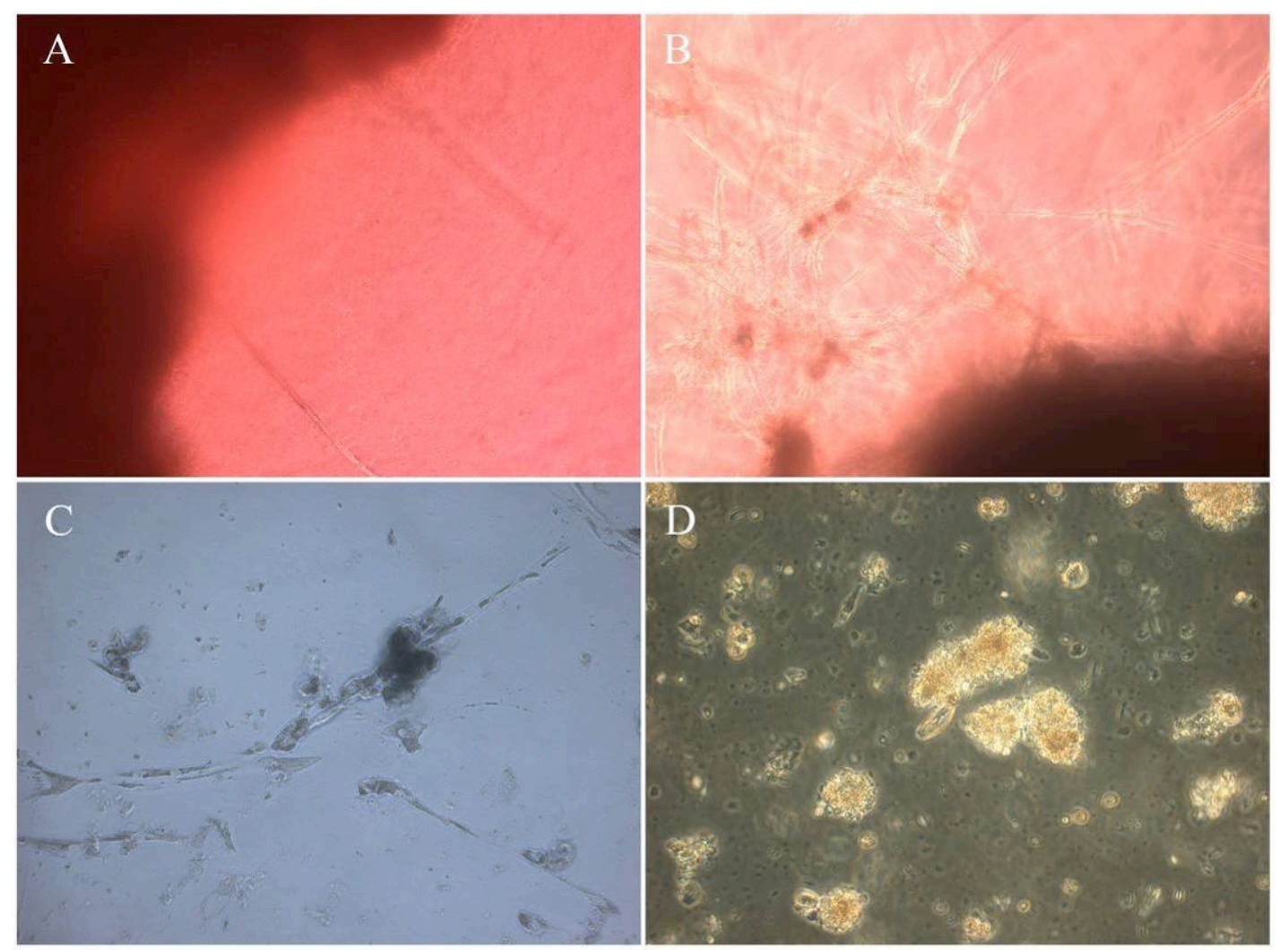

Supplementary Figure 1 


\section{CHAPTER 6: Mesenchymal Stem Cells in Infantile Haemangioma}

\subsection{Introduction and Overview}

An understanding of the cellular and molecular events involved in the life cycle of $\mathrm{IH}$ remains enigmatic, and the precise origin of the fibro-fatty residuum during involution is unknown. The MSCs present within $\mathrm{IH}$ have been presumed to be recruited from adjacent niches and have been proposed to contribute to the fibro-fatty residuum seen during the involuted phase of this tumour $^{39}$.

However, that we have recently demonstrated a neural crest stem cell and mesenchymal associated protein expression profile on the cells of the endothelium of proliferating $\mathrm{IH}$ capillaries described in Chapter 3 of this thesis. Further to this, in the same chapter we have also demonstrated that cells that migrate out from proliferating $\mathrm{IH}$ explants, also express the same markers. We have previously hypothesised that these cells form the downstream definitive mesenchymal lineages that predominate in the fatty tissue seen in the involuted lesions.

This notion that the source of the MSCs that ultimately give rise to the fibro-fatty deposition of involuted $\mathrm{IH}$ is derived from the endothelium of proliferating $\mathrm{IH}$ lesions is a novel concept.

In this chapter I propose that the source of the MSCs that ultimately gives rise to the fibro-fatty deposition of involuted $\mathrm{IH}$ is derived from the cells 
that form the endothelium of the capillaries of proliferating $\mathrm{IH}$ lesions. This is a novel concept that has not previously been proposed.

While the cells of the endothelium have many characteristics of immature endothelial cells (e.g., expression of CD31 and CD34) ${ }^{29}$. I propose that these endothelial-like cells are in fact a primitive population of cells with the capacity to differentiate into both endothelial cells and into terminal mesenchymal derived adipocytes. Such an unorthodox hypothesis raises the question of what factors, if any, are regulating the terminal differentiation into adipocytes. It is in this chapter that I discuss the involvement of pre-adipocyte factor- 1 (Pref- 1$)^{72}$ as one possible factor expressed during proliferation and is lost during involution that may be crucial in regulating terminal mesenchymal differentiation.

This chapter also demonstrates that it is the same cells that migrate out from the proliferating $\mathrm{IH}$ explants, as previously characterised in Chapter 3 , that possess the potential to differentiate into terminal mesenchymal tissues.

The findings of a primitive phenotype of the endothelial layer of proliferating $\mathrm{IH}$ with the ability to differentiate down mesenchymal lineages, yet are inhibited from doing so during proliferation represents a significant paradigm shift in the understanding of $\mathrm{IH}$ as a developmental disorder.

\subsection{Contribution}


The novel concept of the endothelium of proliferating $\mathrm{IH}$ as the source of the adipocytes coupled with the expression of Pref-1 as a mesenchymal differentiation inhibiting protein was conceived by myself. The IHC experiments and the draft for the manuscript was written by myself and edited by my supervisors. The RT-PCR and functional differentiation experiments were performed by Dr Vishvanath.

\subsection{Manuscript: Mesenchymal Stem Cells In Infantile}

\section{Haemangioma}


Original article

\title{
Mesenchymal stem cells in infantile haemangioma
}

\author{
Tinte Itinteang, ${ }^{1,2,3}$ Anasuya Vishvanath, ${ }^{1,2}$ Darren J Day, ${ }^{1,2,3}$ Swee T Tan ${ }^{1,2,3,4}$
}

- An additional table is published online only. To view this file please visit the jouma online (http://jcp.bmj.com).

${ }^{1}$ School of Biological Sciences, Victoria University of Wellington, Wellington, New Zealand ${ }^{2}$ Centre for the Study \& Treatment of Vascular Bithmarks, Wellington Regional Plastic, Maxillofacial \& Burns Unit, Hutt Hospital, Wellington New Zealand

${ }^{3}$ Gillies McIndoe Research Institute, New Zealand ${ }^{4}$ University of Otago, Wellington, New Zealand

Correspondence to

Professor Swee T Tan, Wellington Regional Plastic,

Maxillofacial \& Burns Unit, Hutt Hospital, High Street, Private Bag 31-907, Lower Hutt, New Zealand; swee.tan@ huttvalleydhb.org.nz

$\mathrm{TI}$ and $\mathrm{AV}$ are equal first authors.

D.JD and STT are equal senior

authors.

Part of this work was presented at: the Annual Scientific Meeting, Auckland, New Zealand, 21 November 2008; the 15th International Society for the Study of Vascular Anomalies Workshop. Boston, USA, 20-24 June 2008; and the 16th International Society for the Study of Vascular Anomalies Workshop, Brussels Belgium, 19-21 April 2010.

Accepted 6 December 2010 Published Online First

17 January 2011
ABSTRACT

Background Fibro-fatty deposition commonly occurs during involution of infantile haemangioma $(\mathrm{IH})$. Mesenchymal stem cells have been identified in this tumour and have been proposed to be recruited from the bone marrow and/or adjacent niches, and then give rise to the fibro-fatty tissue. The authors have recently demonstrated that the capillary endothelium of proliferating $\mathrm{IH}$ co-expresses primitive mesodermal, mesenchymal and neural crest markers and proposed that this same endothelium has the ability to give rise to cells of mesenchymal lineage that constitute the fibrofatty deposition.

Methods Immunohistochemistry and real-time RT-PCP were used to further characterise proliferating $\mathrm{IH}$ s and haemangioma explant-derived cells (HaemEDCs).

Results The authors have further confirmed expression of the mesenchymal-associated proteins including preadipocyte factor-1, a mesenchymal differentiation inhibition-associated cytokine. The HaemEDCs could be differentiated into osteoblasts and adipocytes, indicating their functional potential for terminal differentiation.

Discussion The collective expression of neural crest, mesenchymal and mesenchymal differentiation inhibition-associated proteins on the endothelium of proliferating $\mathrm{IH}$ suggests that the cells in the capillary endothelium within the lesion possess the ability to undergo terminal mesenchymal differentiation during the proliferating phase, but are inhibited from doing so.

\section{INTRODUCTION}

Infantile haemangioma (IH) typically exhibits rapid postnatal growth during infancy (proliferative phase) which is characterised by proliferation of endothelial cells ${ }^{1-3}$ and accumulation of other cell types, such as myeloid cells $\mathrm{s}^{4}$ and mesenchymal stem cells (MSCs). ${ }^{6}$ The cellular elements are gradually replaced by fibro-fatty tissue during the next $1-5$ years (involuting phase), with continued improvement up to 12 years of age (involuted phase), often leaving a fibro-fatty residuum. ${ }^{1-3} 7$

Despite considerable progress towards the understanding of the cellular and molecular events involved in its life cycle, IH remains enigmatic, and the precise origin of the fibro-fatty residuum is unknown. The MSCs observed in $\mathrm{IH}$ have been proposed to be recruited from the bone marrow and/or adjacent niches, and then contribute to the fibro-fatty differentiation seen during the involuted phase of this tumour. ${ }^{6}$

We have recently shown expression of neural crest and mesenchymal-associated proteins on the cells of the endothelium of the capillaries of proliferating $\mathrm{IH}^{8}{ }^{8}$ We have also demonstrated that cells that emanate from proliferating IH explants retain the same markers, and we hypothesise that these cells form the downstream adipocytes that predominate in the fibro-fatty tissue of involuted lesions. $^{8}$

In this study, we identified cells expressing mesenchymal-associated proteins within proliferating $\mathrm{IH}$, as well as isolating and characterising the cells obtained from proliferating IH explants, to investigate their MSC properties. We also investigated the expression of preadipocyte factor- 1 (Pref-1), also known as DLK, an epidermal growth factor-like protein that is crucial in the prevention of terminal adipogenesis.

We further investigated the ability of cells derived from proliferating $\mathrm{IH}$ explants that coexpress endothelial, neural crest and mesenchymal markers to form terminal mesenchymal cells.

\section{MATERIALS AND METHODS}

\section{IH tissues}

All IH tissues were obtained from patients according to a protocol approved by the Wellington Regional Ethics Committee.

\section{Immunolabelling}

Biopsy specimens from six patients with proliferating $\mathrm{IH}$, aged $3-10$ months (mean 6 ), and three patients, aged 6,7 and 9 years with involuted $\mathrm{IH}$, were formalin-fixed and paraffin embedded using standard procedures. H\&E and immunohistochemical (IHC) staining were performed on $4 \mu \mathrm{m}$ paraffin-embedded serial sections. Immunolabelling was performed as previously described ${ }^{8}$ with primary antibodies specific for human Pref-1 (Abcam, MA; 1:200 dilution) and CD34 (Dako Corp, Glostrup, Denmark; 1:200 dilution). Negative controls used in this study included staining of paraffin sections of tissue not expected to be immunoreactive with the same series of antibodies including adult human subcutaneous tissue, and also by omission of the primary antibodies when selected proliferating IHs were being stained. These controls showed the expected staining pattern consistent with the previously reported specificity for the antibodies used. ${ }^{9}$

\section{IH tissue explant culture}

Tissue pieces from five patients with proliferating $\mathrm{IH}$ and two patients with involuted $\mathrm{IH}$ were embedded in a thin layer of fibrin gel and cultured as explants as described by Tan et al, ${ }^{10}$ and grown in the presence of $10 \%$ foetal calf serum (Invitrogen, Auckland, New Zealand). Capillary outgrowth was recorded during the culture period by capturing images with an Olympus IX51 inverted microscope (upgraded to IX71) fitted with phase-contrast objectives and a Colour View 1 camera (Olympus Corporation, Tokyo, Japan). 
Isolation and culture of haemangioma explant-derived cells (HaemEDCs)

Tissue pieces were grown as explants as previously described. ${ }^{8}$ After 3 weeks in culture, the tissue pieces were removed from the fibrin matrix, and cells that had emanated from the tissue were recovered by protease digestion. ${ }^{8}$

Mesenchymal differentiation of HaemEDCs

HaemEDCs recovered from the IH explants were differentiated towards adipocytes or osteoblasts in RPMI 1640 medium using previously described culture conditions. ${ }^{11}$ Differentiation medium was replaced every 2-3 days. After induction for 2 weeks, calcium deposition was detected by staining with Alizarin Red ${ }^{12}$ and lipid-laden vesicles within adipocytes by OilRed-O staining. ${ }^{13}$

\section{Immunocytochemistry}

HaemEDCs were grown on $13 \mathrm{~mm}$-diameter borosilicate glass coverslips (Biolab, Auckland, New Zealand) and processed for immunocytochemistry. ${ }^{9}$ Detection of primary antibodies was performed overnight at $4^{\circ} \mathrm{C}$ using one of the following antibodies diluted as indicated: mouse anti- $\alpha$-SMA (1:100), mouse anti-vimentin (1:100), rabbit anti-GLUT-1 (1:100), mouse antiCD34 (1:100), all from Dako Corp. Bound antibodies were detected as for IHC labelling.

RNA extraction and quantitative real-time PCR (qRT-PCR)

RNA was isolated from $\sim 1 \times 10^{6}$ cells with Trizol (Invitrogen) according to the manufacturer's instructions, except for the final ethanol precipitation step, in which the aqueous phase was mixed with an equal volume of $70 \%$ ethanol and then applied to a High Pure Tissue Extraction Column (Roche Diagnostics, Auckland, NZ). Subsequent washes and elution from the column were performed according to the manufacturer's instructions. Approximately 200-500 ng RNA was reversetranscribed using Superscript III (Invitrogen) and oligo-dT $(2.5 \mu \mathrm{M})$ as primer, in a volume of $20 \mu \mathrm{l}$, according to the manufacturer's instructions. qRT-PCR amplification was performed on $10 \%$ of the reverse transcription using Platinum SYBR green qPCR supermix-UDG (Invitrogen), and gene-specific primers $(0.1 \mu \mathrm{M})$ for the mesenchymal-associated markers, CD29, CD44, CD90 and Pref-1, were appropriately diluted to a final reaction volume of $20 \mu \mathrm{l}$. Fluorescein (10 nM; Invitrogen) was included to allow automated detection of samples in an iQ qPCR system (Bio-Rad Laboratories, Hercules, CA) running the manufacturer's software. The threshold-cycle (Ct) was detected using the software and manually adjusted as appropriate. All reactions were performed in duplicate, and each experiment included a no-template (water) control and amplification from an equivalent amount of RNA that had not been reverse-transcribed. Amplification was achieved with an initial denaturation at $95^{\circ} \mathrm{C}$ for $30 \mathrm{~s}$, followed by 35 cycles of denaturation at $95^{\circ} \mathrm{C}$ for $15 \mathrm{~s}$, annealing at $60^{\circ} \mathrm{C}$ for $20 \mathrm{~s}$ and extensions at $72^{\circ} \mathrm{C}$ for $20 \mathrm{~s}$. Data were collected from both the annealing and extension steps, and a melt-curve analysis was performed to confirm the absence of spurious amplification products. Agarose gel electrophoresis was performed on selected amplifications from each run to confirm that products of the expected size were amplified. All the primers (online supplemental table 1) were obtained from Invitrogen. Changes in gene expression are reported relative to that for glyceraldehyde-3-phospate dehydrogenase (GAPDH) as a $\Delta \mathrm{Ct}$ value or, when comparing treatment groups, as $\Delta \mathrm{Ct}$ values \pm SEM.

\section{RESULTS}

Immunohistochemistry on haemangioma tissues

$\mathrm{We}^{14}$ and others ${ }^{15}{ }^{16}$ have demonstrated two distinct populations of cells within capillaries of proliferating $\mathrm{IH}$, with an inner phenotypic endothelial layer and the outer concentric pericyte layer expressing $\alpha$-SMA.

We have recently demonstrated cells expressing the neural crest-associated surface receptor (p75) and the mesenchymalassociated proteins (CD29 and vimentin) to the endothelialphenotypic layer, distinct from the outer pericyte layer which expressed $\alpha$-SMA. ${ }^{14}$

IHC staining was undertaken to determine if the same primitive cells in the endothelium of $\mathrm{IH}$ that co-expressed neural crest and MSC markers also expressed Pref-1, an epidermal-like cytokine that has been shown to inhibit adipocytic differentiation. ${ }^{17}$ Strong expression of Pref-1 (figure $1 \mathrm{~A}$, red) was observed on the endothelium, identified by immunoreactivity (IR) for $\mathrm{CD} 34$ (figure 1A, green), of all the capillaries within the proliferating IHs. This pattern of IR for Pref-1 in the endothelium of proliferating IH capillaries was lost in the mature microvessels of involuted lesions (figure $1 \mathrm{~B}$, red), with the endothelial layer identified by IR for CD34 (figure 1B, green). Immunostaining for vessels of adult subcutaneous tissues, as controls, revealed similar staining patterns for Pref-1 to that seen in involuted IH vessels, with IR for CD34 (figure 1C, green) and minimal IR for Pref-1 (figure 1C, red).
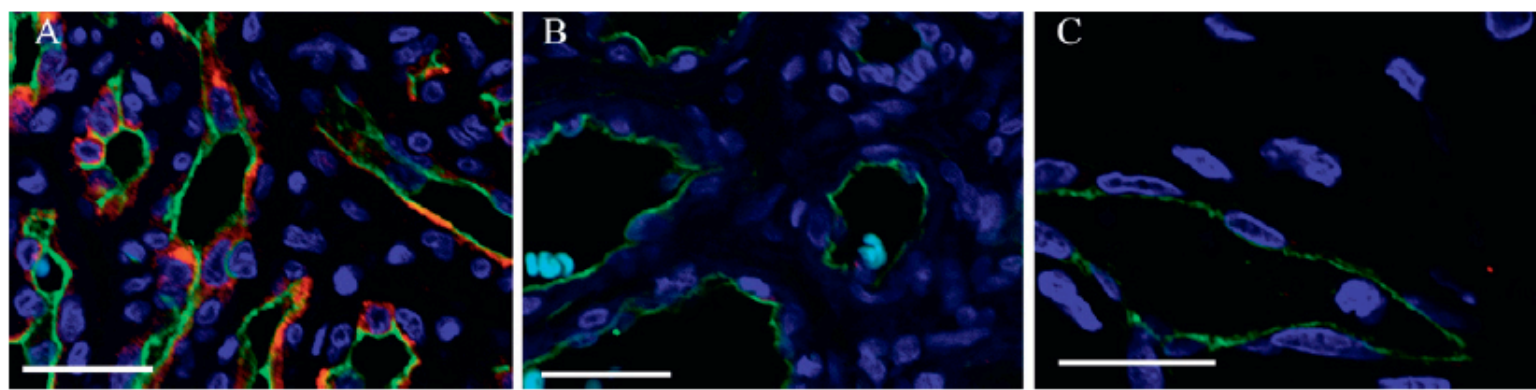

Figure 1 Immunohistochemical staining of representative sections. (A) Endothelium of proliferating infantile haemangioma (IH) capillaries shows strong immunoreactivity (IR) for CD34 (green) and Pref-1 (red). (B) Cells forming the microvessel endothelium in involuted IH show IR for CD34 (green) with minimal IR for Pref-1 (red). (C) shows the microvessels in adult subcutaneous tissue, identified by IR to CD34 (green), with no IR for Pref-1 (red). The cell nuclei in all panels are counter stained with 6'-diamidino-2-phenylindole (DAPI) (blue). Scale bar $50 \mu \mathrm{m}$ for (A,B) and $25 \mu \mathrm{m}$ for (C). 
Original article

\section{Determination of mRNA expression by qRT-PCR}

To corroborate the IHC data on $\mathrm{IH}$ tissues presented in figure 1 , mRNA expression for $\mathrm{CD} 29^{14}$ and the cytokine Pref- 1 in proliferating IH biopsy specimens was determined by qRT-PCR. In addition, mRNA expression for the mesenchymal cell markers, $\mathrm{CD} 44^{18}$ and $\mathrm{CD} 900^{6}$ was included even though these were not used for the IHC study. Table 1 shows the abundance of these transcripts relative to that for GAPDH and confirms the abundant expression of the transcripts coding for the proteins detected by $\mathrm{IHC}$ staining in proliferating $\mathrm{IH}$.

We have previously shown that cells isolated from the outgrowths that form when $\mathrm{IH}$ biopsy specimens are grown as explants express endothelial, mesenchymal and neural crestassociated proteins. ${ }^{8}$ Table 2 shows the relative abundance of mRNA transcripts for $\mathrm{CD} 29, \mathrm{CD} 44^{18}$ and $\mathrm{CD} 90^{6}$ determined after increasing numbers of passage for HaemEDCs. The HaemEDCs grow slowly in culture such that passage 3 represents approximately 3 weeks in culture and passage 9, 6 months. The 3T3 cells and human umbilical vein endothelial cells (HUVECs) used for comparison had been cultured for a similar period of time but required more passaging. Table 2 shows that the HaemEDCs isolated maintain expression of mesenchymal markers even after extended periods of culture.

\section{Differentiation of HaemEDCs}

To determine the functional ability of the HaemEDCs to form definitive mesenchymal-derived cells, we investigated the ability of these cells to differentiate into adipocytes and osteoblasts.

HaemEDCs could be differentiated to form lipid-laden adipocytes (figure 2A) that stained with Oil-Red-O (figure 2B). HaemEDCs (figure 2C) could also be differentiated into osteoblastic cells (figure 2D) as determined by Alizarin Red staining indicating calcification of the extracellular matrix. Extended (5 months) culturing of the HaemEDCs resulted in spontaneous differentiation of the majority of cells to lipid-laden adipocytes that adhered to the bottom of the culture wells (data not shown) even in the absence of the specific factors used to induce adipocyte formation as in figure $2 \mathrm{~B}$.

In addition, the differentiation into adipocytes and osteoblasts was supported by the expression of mRNA for peroxisome proliferator-activated receptor (PPAR- $\gamma$; a key regulatory gene of adipogenesis $\left.{ }^{19}\right)$ and osteonectin, ${ }^{20}$ respectively. Table 3 shows the relative change in expression levels of these genes in undifferentiated HaemEDC isolates compared with the corresponding differentiated cells. mRNA expression is reported relative to GAPDH $(\Delta \mathrm{C} t)$, and as $\Delta \mathrm{Ct}$ for undifferentiated versus differentiated cells. Expression of PPAR- $\gamma$ increased as expected $(\sim 16-$ fold), whereas that of osteonectin did not alter significantly.

\section{DISCUSSION}

It has previously been suggested that MSCs identified in IH may be recruited into the tumour from the bone marrow and/or from

Table 1 Gene expression profile of proliferating infantile haemangioma (IH) tissues

\begin{tabular}{ll}
\hline mRNA transcript & $\Delta \mathbf{C t}$ \\
\hline CD29 & $2.45 \pm 0.19$ \\
CD44 & $2.49 \pm 0.95$ \\
CD90 & $3.63 \pm 1.09$ \\
Pref-1 & $7.30 \pm 0.54$ \\
\hline mRNA expression for CD29, CD44, CD90 and Pref-1 was analysed by qRT-PCR from five \\
proliferating IH biopsy specimens. Expression of transcripts was determined relative to \\
glyceraldehyde-3-phosphate dehydrogenase ( $\Delta$ Ct). Values are mean $\triangle \mathrm{Ct}$ obtained for each \\
gene \pm SEM.
\end{tabular}

Table 2 mRNA characterisation of haemangioma explant-derived cells (HaemEDCs)

\begin{tabular}{llll}
\hline Cell type & CD29 & CD44 & CD90 \\
\hline HaemEDCs-P1 (P3) & 2.9 & 4.4 & 5.2 \\
HaemEDCs-P1 (P9) & 2.9 & 3.7 & 4.6 \\
HaemEDCs-P2 (P3) & 1.9 & 3.0 & 2.8 \\
HaemEDCs-P3 (P3) & 1.0 & 2.5 & 2.2 \\
HaemEDCs-P4 (P3) & 1.2 & 3.7 & 2.6 \\
\hline
\end{tabular}

mRNA expression for the mesenchymal stem cell-specific markers, CD29, CD44 and CD90, was analysed from four proliferating infantile haemangioma (IH) cell isolates (P1-P4). RNA was analysed from four proliferating infantile haemangioma $(\mathrm{IH})$ cell isolates $(\mathrm{P} 1-\mathrm{P} 4)$.
was extracted from $1 \times 10^{5}$ cells, reverse-transcribed into $\mathrm{CDNA}$, and analysed by was extracted from $1 \times 10^{3}$ cells, reverse-transcribed into CDNA, and analysed by
quantitative real-time (qRT-PCR. Expression of transcripts was determined relative to quantitative real-time (qRT-PCR. Expression of transcripts was determined relative to
glyceraldehyde-3-phosphate dehydrogenase ( $\Delta \mathrm{Ct}$ ). The table gives the $\Delta$ Ct values obtained for each gene. The passage number is given in parentheses; '-' shows no amplification and therefore the absence of mRNA transcript. The mean $\triangle \mathrm{Ct}$ value $( \pm$ SEM $)$ across the four IH cell isolates ( $P 3)$ for $\mathrm{CD} 29, \mathrm{CD} 44$ and $\mathrm{CD} 90$ was $1.75( \pm 0.4), 3.4( \pm 0.4)$ and $3.2( \pm 0.7)$, respectively.

the adjacent stem cell niches ${ }^{6}$. In this study, we have confirmed our previous data showing the expression of multiple proteins associated with cells of the mesenchymal lineage within $\mathrm{IH}$. Here we show that $\mathrm{CD} 29,{ }^{9}$ a cell surface marker associated with MSCs; ${ }^{6} 21$ and vimentin, ${ }^{9}$ an intermediate filament associated with cells of the mesenchymal lineage. ${ }^{21}{ }^{22}$ In this study we have also demonstrated the expression of Pref-1, a member of the epidermal growth factor-like family which prevents terminal adipocyte differentiation in pre-mesenchymal cells, by the cells forming the endothelial layer of the capillaries in proliferating IHs. The IHC findings are supported by the qRT-PCR experiments, which show abundant expression of mRNA coding for these mesenchymal-associated proteins, even after extended periods of culture. We have also shown that the cells emanating from the $\mathrm{IH}$ explant culture express the same mesenchymalassociated proteins ${ }^{8}$ and mRNA as that found within the lesions. These data, combined with the functional ability of these cells to differentiate into adipocytes and osteoblasts, collectively confirms the presence of an abundant MSC population within proliferating $\mathrm{IH}$. The expression of the MSC markers and mesenchymal-associated proteins on the cells of the capillary endothelium of proliferating IH supports the hypothesis that the endothelium is the origin of the fibro-fatty material seen during spontaneous involution, and is supported by the loss of Pref- 1 expression in the endothelium of microvessels as the lesions involute.

We have demonstrated a neural crest stem cell with primitive mesoderm phenotype in both the endothelium of the capillaries of proliferating $\mathrm{IH}$ and the cells migrating out of the explant tissue culture. We have also recently presented data to support a haemogenic endothelium phenotype of the capillaries of proliferating $\mathrm{IH}^{9}$ These same neural crest phenotypic cells also express proteins associated with cells of the endothelial and mesenchymal lineages recently shown by $\mathrm{s}^{8}$ and others. ${ }^{10}{ }^{18}$ It is intriguing and unexpected that the cells of the capillary endothelium express markers identified with the primitive streak (brachyury) and neural crest (p75), as well as the more mature mesenchymal, endothelial and haematopoietic lineages. Here we provide functional data in support of our recent findings that the cells of the endothelium with the neural crest stem cell phenotype are the same as cells isolated from cultured explants that have the ability to terminally differentiate down the mesenchymal lineage. Previous reports highlighting neural crest phenotypic cells derived from embryonic stem cells as being the precursors to cells of the mesenchymal lineage $e^{22}$ are in concordance with our findings and point to a very primitive origin for $\mathrm{IH}$. The finding that explants of the involuted $\mathrm{IH}$ lesions used in this study did not exhibit outgrowth after 
Figure 2 Haemangioma explantderived cells (HaemEDCs) isolated from proliferating infantile haemangiomas (IHs) were plated on to glass coverslips at $2 \times 10^{4} \mathrm{cells} / \mathrm{cm}^{2}$ and cultured in osteoblastic or adipocytic differentiation medium. HaemEDCs from proliferating IHs were readily differentiated into lipidladen adipocytes. (A) A phase-contrast image of HaemEDCs differentiated into adipocytes. (B) Cytoplasmic lipid-filled vacuoles were identified by staining with Oil-Red-0. (C) HaemEDCs from proliferating $\mathrm{IH}$ differentiated into osteoblastic cells. The formation of calcium deposits was confirmed by Alizarin Red staining (D). Scale bars: $50 \mu \mathrm{m}$.
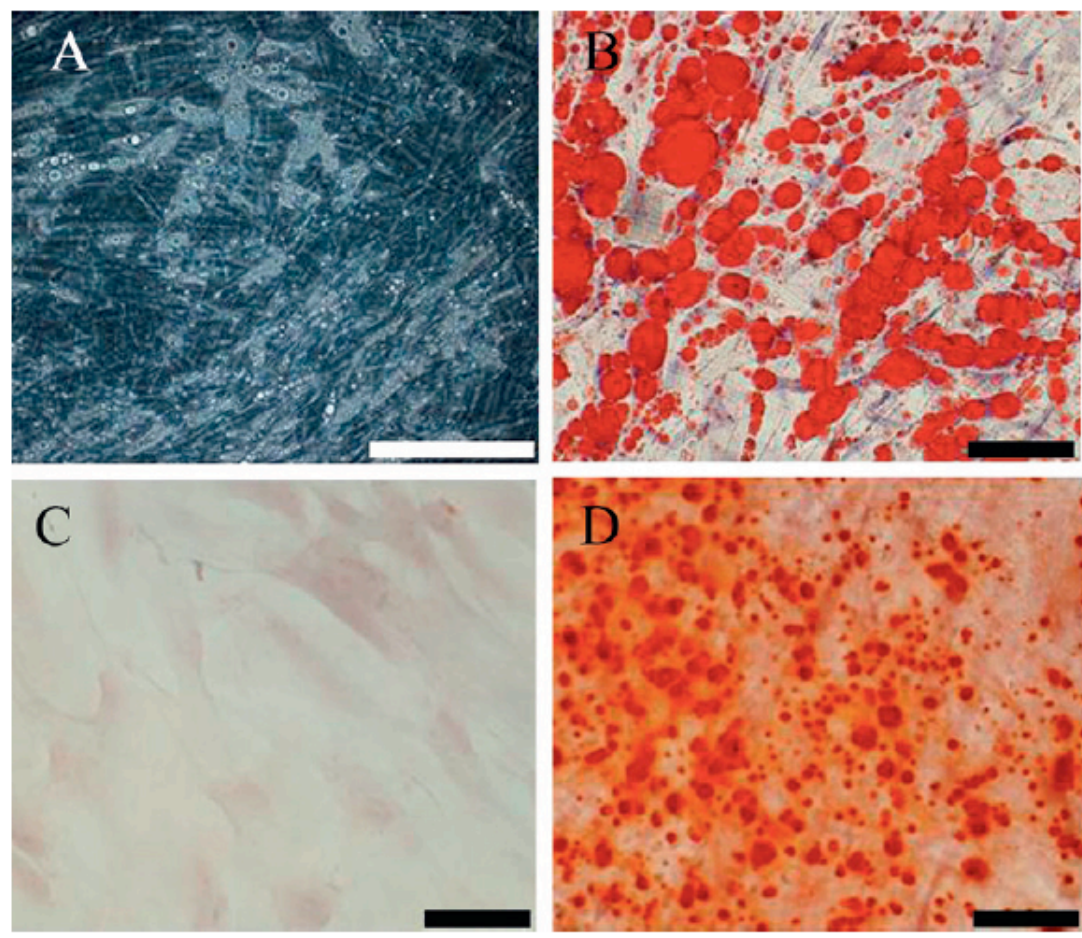

2 weeks in culture is consistent with our previous observation. ${ }^{10}$ It probably reflects the paucity or absence of immature capillaries within involuted IHs.

Pref- 1 is a pre-adipocyte marker, ${ }^{23}$ and there is strong evidence to support its role in preventing terminal adipocyte differentiation. ${ }^{24}$ It has been shown that, in pre-mesenchymal cells, Pref- 1 exerts some of its inhibitory effect on adipogenesis via PPAR- $\gamma$, which is an important adipocyte differentiation factor. ${ }^{17}$ This is consistent with our data showing expression of Pref-1 mRNA and upregulation of PPAR- $\gamma$ after differentiation of HaemEDCs towards adipocytes. The collective expression of neural crest, MSCs and mesenchymal differentiation inhibition-associated proteins on the endothelium of proliferating $\mathrm{IH}$ is consistent with the cells of the endothelium being of very primitive origin, and they are inhibited from terminal mesenchymal differentiation by the action of Pref- 1 during the proliferating phase. We have also recently suggested that the high levels of angioten sin II

Table 3 Change in peroxisome proliferator-activated receptor (PPAR- $\gamma$ ) and osteonectin mRNA expression in haemangioma explant-derived cells (HaemEDCs) before and after differentiation

\begin{tabular}{llc}
\hline & $\Delta$ Ct PPAR- $\gamma$ & $\Delta$ Ct osteonectin \\
\hline Haem-P1 & 4.7 & 3.4 \\
Haem-P2 & 4.3 & -1.5 \\
Haem-P3 & 5.5 & 1.7 \\
Haem-P4 & 4.9 & -0.2 \\
Haem-P5 & 2.9 & -1.0 \\
Mean difference in gene expression & 4.7 & 0.5 \\
SEM & 0.4 & 0.9 \\
\hline
\end{tabular}

Cell isolates from explant culture of five proliferating infantile haemangiomas (His), designated HaemEDCs-1, HaemEDCs-2, HaemEDCs-3, HaemEDCs -4 and HaemEDCs-5, were differentiated into adipocytes and osteoblasts. Quantitative real-time (qRT)-PCR was performed to identify changes in PPAR- $\gamma$ and osteonectin mRNA expression in undifferentiated cells versus differentiated cells $(\Delta \Delta C \mathrm{Ct})$. during infancy have the ability to inhibit terminal mesenchymal differentiation in proliferating $\mathrm{IH}^{11}$ The ability of HaemEDCs to form adipocytes in culture, in the absence of differentiation medium after extended periods in culture highlights adipogenesis as a possible default pathway in the absence of mesenchymal differentiation-inhibiting cytokines. The exact roles of Pref- 1 and angiotensin II in adipogenesis regulation during IH progression remain to be conclusively determined.

The expression of both mesenchymal-associated proteins and endothelial markers ${ }^{18}{ }^{22}$ by the same cells has not been demonstrated previously, except in cells of the primitive mesoderm. ${ }^{25}$ The latter are precursors to both mesenchymal and haematopoietic lineages. ${ }^{26-28}$ This study therefore adds to an increasing body of evidence supporting a primitive mesodermal origin for the endothelium of capillaries in proliferating $\mathrm{IH}$, and identifies cells with a novel phenotype that express haematopoietic and endothelial markers with the ability to differentiate into definitive mesenchymal cells.

We suggest that proliferating $\mathrm{IH}$ possesses innate mesenchymal precursors that are able to perform, or may account for, the terminal fibro-fatty differentiation that occurs during involution. The contribution of MSCs from adjacent niches and the bone marrow to the fibro-fatty deposition ${ }^{6}$ remains to be

Take-home message

The cells forming the endothelium of IH are primitive in origin and express mesenchymal stem cell markers and the mesenchymal differentiation inhibition factor Pref-1. Cells isolated from IH retain the ability to undergo terminal mesenchymal differentiation after extended periods of culture. 
Original article

conclusively determined. However, in the light of these results, we feel that it is unlikely to be the major source, given the abundance of pre-mesenchymal cells that undergo differentiation to adipocytes by default innately from within proliferating $\mathrm{IH}$.

$\mathrm{IH}$ provides a unique model system for studying primitive pathways of mesenchymal differentiation from a novel cell phenotype that co-expresses haematopoietic, endothelial and mesenchymal markers.

Funding This work is supported by grants from the Wellington Regional Plastic Surgery Unit Research \& Education Trust, the Wellington Medical Research Foundation, the Surgical Research Trust, Pub Charities and the Cancer Society of New Zealand. AV was supported, in part, by the Cancer Society of New Zealand. TI was supported by the Royal Australasian College of Surgeons' Foundation for Surgery Scholarship.

\section{Competing interests None.}

Ethics approval This study was conducted with the approval of the Wellington Regional Ethics Committee.

Provenance and peer review Not commissioned; extemally peer reviewed.

\section{REFERENCES}

1. North PE, Waner M. Mizeracki A, et al. Glut1: a newly discovered immunohistochemical marker for jivenile hemangiomas. Hum Pathol immunohistochen $2000 ; 31: 11-22$

2. Frieden IJ, Eichenfield LF, Esterly NB, et al. Guidelines of care for hemangiomas of infancy. American Academy of Dermatology Guidelines/Outcomes Committee. J Am Acad Dermatol 1997; 37:631-7.

3. Tan ST, Velickovic M, Ruger BM, et al. Cellular and extracellular markers of hemangioma. Plast Reconstr Surg 2000:106.529-37.

4. Fitter MR, Reinisch J, Friedlander SF, et al. Myeloid cells in infantile hemangioma. Am J Pathol 2006:168:621-8.

5. Tan ST, Wallis RA, He Y, et al. Mast cells and hemangioma. Plast Reconstr Surg 2004:113:999-1011.

6. Yu Y, Fuhr J, Boye E, et al. Mesenchymal stem cells and adipogenesis in hemangioma involution. Stem Cells 2006;24:1605-12.

7. Tan ST, Itinteang T, Leadbitter P. Low-dose propranolal for infantile haemangioma J Plast Reconstr Aesth Surg Published Online First: 2010 Jul 7. doi:10.1016/j. bips.2010.06.010.

8. Itinteang $\mathbf{T}$, Tan ST, Brasch H, et al. Primitive mesodermal cells with a neural crest stem cell phenotype predominate prolfferating infantile haemangioma. J Clin Pathol 2010:63:771-6.

9. Itinteang $\mathbf{T}$, Tan ST, Brasch $\mathrm{H}$, et al. Haemogenic endothelium in infantile haemangioma. J Clin Pathol 2010;63:982-6.
10. Tan ST, Hasan Q, Velickovic M, et al. A novel in vitro human model of hemangioma. Mod Pathol 2000;13:92-9.

11. Itinteang T, Brasch HD, Tan ST, et al. Expression of components of the reninangiotensin system in proliferating infantile haemangioma may account for the propranolol- induced accelerated irvolution. J Plast Reconstr Aesth Surg Published Online First: 2010 Sep 24. doi:10.1016/.j.jps.2010.08.039.

12. D'Ippolito G, Diabira S, Howard GA et al. Marrow-isolated adult multilineage inducible (MIAMI) cells, a unique population of postnatal young and old human cells with extensive expansion and differentiation potential. J Cel/ Sci 2004;117:2971-81.

13. Reyes $\mathbf{M}$, Lund $\mathrm{T}$, Lervik $\mathrm{T}$, et al. Purification and ex vivo expansion of postnatal human manow mesodermal progenitor cells. Blood 2001;98:2615-25.

14. Takahashi K, Mulliken JB, Kozakewich HP, et al. Cellular markers that distinguish the phases of hemangioma during infancy and childhood. J Clin Irvest 1994:93:2357-64.

15. Smoller BR, Alfelberg DB. Infantile (juvenile) capillary hemangioma: a tumor of heterogeneous cellular elements. J Cutan Pathol 1993;20:330-6.

16. Pantoja C, Huff J, Yamamoto KR. Glucocorticoid signaling defines a novel commitment state during adipogenesis in vitro. Mol Biol Cell 2008-19:4032-41.

17. O'Donoghue K, Fisk NM. Fetal stem cells. Best Pract Res Clin Obstet Gynaecol 2004:18:853-75

18. Ritter MR, Dorrell MI, Edmonds J, et al. Insulin-like growth factor 2 and potential regulators of hemangioma growth and irvolution identified by large-scale expression analysis. Proc Natl Acad Sci U S A 2002;99:7455-60.

19. Wu Z, Bucher N, Farmer S. Induction of peroxisome proliferator-activated receptor gamma during the corversion of $3 \mathrm{~T} 3$ fibroblasts into adipocytes is mediated by C/EBPbeta, C/EBPdelta, and glucocorticoids. Mol Cell Biol 1996;16:4128-36.

20. Jundt G, Berghãuser $\mathrm{K}$, Termine J, et al. Osteonectin-a differentiation marker of bone cells. Cell Tissue Res 1987:248:409-15.

21. Lindolfo da Siva M, Amold IC, Nance Beyer N. In search of the in vivo identity of mesenchymal stem cells. Stem Cells 2008:26:2287-99.

22. Lee G, Kim H, Elkabetz Y, et al. Isolation and directed differentiation of neural crest stem cells derived from human embryonic stem cells. Nat Biotechnol 2007:25:1468-75.

23. Kim K, Kim J, Wang Y, et al. Pref-1 (preadipocyte factor 1) activates the mek/ extracellular signal-regulated kinase pattwway to inhibit adipocyte differentiation. Mo Cell Biol 2007;27:2294-308.

24. Sul HS. Minireview: Pref-1: role in adipogenesis and mesenchymal cell fate. Mol Endocrinal 2009:23:1717-25.

25. Dent JA, Polson AG, Klymkowsky MW. A whole-mount immunocytochemical analysis of the expression of the intermediate flament protein vimentin in xenpus. Development 1989:105:61-74

26. Olmsted-Davis EA, Gugala Z, Camargo F, et al. Primitive adult hematopoietic stem cells can function as osteoblast precursors. Proc Nat Acad Sä US A 2003;100:15877-82

27. Dominici M, Pritchard C, Garlits JE, et al. Hematopoietic cells and osteoblasts are derived from a common marrow progenitor after bone marrow transplantation. Proc Natt Acad Sci U S A 2004:101:11761-6.

28. Pimanda JE, Siberstein L, Dominici M, et al. Transcriptional link between blood and bone: the stem cell leukemia gene and its +19 stem cell enhancer are active in bone cells. Mol Cell Biol 2006:26:2615-25. 


\section{Chapter 7: Osteoprotegerin and Receptor for Nuclear Factor $\kappa \beta$ in Infantile Haemangioma}

\subsection{Introduction and Overview}

The expression of OPG and its ligand TRAIL in IH was previously demonstrated by Vishvanath et $a l^{11}$ who suggest that OPG was part of a critical pathway in the maintenance of anti-apoptosis seen during cellular accumulation of the proliferating phase of $\mathrm{IH}$. Further to this OPG, has also been demonstrated to be critical in the recruitment and differentiation of osteoclasts via its actions on RANK, acting on RANKL to inhibit osteoclastic bone resorption, presumably through inhibition of osteoclast differentiation ${ }^{47}$.

This chapter discusses the novel implications of OPG and TRAIL in anti-apoptosis within $\mathrm{IH}$. Interesting, however, is the observation that RANKLpositive cells are located within the interstitium of these proliferating lesions. This unique and exclusive expression pattern of the expression of RANKL highlights a potential primitive macrophage population within proliferating $\mathrm{IH}$. As to whether these cells are recruited into the tumour or whether they are a population derived from the haemogenic endothelium remains to be conclusively determined.

\subsection{Contribution}


Demonstration of the presence of RANKL-positive cells and its incorporation into the body of the manuscript were performed by me, all other experiments in this manuscript and the concept of OPG and TRAIL together with the drafting of the manuscript was done by Dr Vishvanath. Final editing was done by my supervisors. 
7.3. Manuscript: Infantile Haemangioma Expresses Tumour Necrosis Factor-Related Apoptosis-Inducing Ligand (TRAIL), TRAIL Receptors, Osteoprotegerin And Receptor Activator For Nuclear Factor K $\beta$ Ligand (RANKL) 


\title{
Infantile haemangioma expresses tumour necrosis factor-related apoptosis-inducing ligand (TRAIL), TRAIL receptors, osteoprotegerin and receptor activator for nuclear factor $\kappa B$ ligand $(R A N K L)^{\dagger}$
}

\author{
Anasuya Vishvanath, ${ }^{1,2}$ Tinte Itinteang, ${ }^{1-3}$ Swee T Tan ${ }^{1-4 *}$ \& Darren J Day ${ }^{1-3}$ \\ ${ }^{1}$ School of Biological Sciences, Victoria University of Wellington, ${ }^{2}$ Centre for the Study and Treatment of Vascular \\ Birthmarks, Wellington Regional Plastic, Maxillofacial and Burns Unit, Hutt Hospital, ${ }^{3}$ Gillies McIndoe Research Institute, \\ and ${ }^{4}$ Wellington School of Medicine and Health Sciences, University of Otago, Wellington, New Zealand \\ Date of submission 26 October 2010 \\ Accepted for publication 1 February 2011 \\ Vishvanath A, Itinteang T, Tan S T \& Day D J \\ (2011) Histopathology \\ Infantile haemangioma expresses tumour necrosis factor-related apoptosis-inducing ligand \\ (TRAIL), TRAIL receptors, osteoprotegerin and receptor activator for nuclear factor $\mathbf{\kappa} \mathrm{B}$ ligand \\ (RANKL)
}

Aims: To investigate the expression of tumour necrosis factor-related apoptosis-inducing ligand (TRAIL) and its receptors and decoy receptors, including osteoprotegerin $(\mathrm{OPG})$ in infantile haemangioma $(\mathrm{IH})$.

Methods and results: Immunostaining, Western blotting and quantitative reverse transcription-polymerase chain reaction (qRT-PCR) were used on $\mathrm{IH}$ biopsies and haemangioma explant-derived cells (HaemEDCs). TRAIL and its receptors and decoy receptors, including $\mathrm{OPG}$, are expressed in proliferating $\mathrm{IH}$ tissues and in HaemEDCs. Cells forming the endothelium of immature capillaries of proliferating IHs express abundant OPG and show punctate von Willebrand Factor (vWF) staining. As the cells mature and assume the characteristic of endothelial cells they increase expression of $\mathrm{vWF}$, but lose expression of OPG. The endothelium of IH shows minimal expression of receptor activator for nuclear factor $\mathbf{\kappa B}$ ligand (RANKL) compared with a small population of RANKL-positive cells located within the interstitium between microvessels. Proliferating HaemEDCs express significantly higher levels of OPG and decoy receptor 2 than the matched tissue samples. Increased OPG expression is detected in the extracellular matrix and in the conditioned medium of HaemEDCs.

Conclusions: Our data suggest that OPG through the TRAIL pathway, but not the RANKL pathway, plays a role in regulating anti-apoptosis during the development of $\mathrm{IH}$.

Keywords: infantile haemangioma, involution, osteoprotegerin, RANKL, RANK, TRAIL, TRAIL receptors

Abbreviations: DR, death receptor; HaemEDCs, haemangioma explant-derived cells; IH, infantile haemangioma; OPG, osteoprotegerin; RANKL, receptor activator for nuclear factor $\mathrm{\kappa B}$ ligand; TRAIL, tumour necrosis factor-related apoptosis-inducing ligand; vWF, von Willebrand factor

Address for correspondence: Professor S T Tan, MBBS, FRACS, PhD, Wellington Regional Plastic, Maxillofacial and Burns Unit, Hutt Hospital, High Street, Private Bag 31-907, Lower Hutt, New Zealand. email: swee.tan@huttvalleydhb.org.nz

These senior authors contributed equally to this study.

${ }^{\dagger}$ This paper was presented in part at the 15 th International Society for the Study of Vascular Anomalies Workshop, Boston, USA, 20-24 June 2008; the New Zealand Association of Plastic Surgeons' Annual Scientific Meeting. Auckland, New Zealand, 21 November 2008; and the 16th International Society for the Study of Vascular Anomalies Workshop, Brussels, Belgium, 19-21 April 2010. 


\section{Introduction}

Tumour necrosis factor-related apoptosis-inducing ligand (TRAIL), also known as Apo2L, is a membrane protein that is cleaved from the cell surface to form a soluble ligand that can induce apoptosis in a large number of cancer cell lines. ${ }^{1-3}$ There are five known TRAIL receptors (TRAILRs) consisting of two transmembrane death receptors, DR4 and DR 5, also known as TRAIL-R1 and TRAIL-R2, respectively; two transmembrane decoy receptors, DcR1 and DcR2; and a soluble decoy receptor, osteoprotegerin (OPG), ${ }^{4}$ that is thought to protect cells from TRAIL-induced apoptosis. ${ }^{2,5}$ There is a growing body of evidence supporting a role for TRAIL and OPG in vascular biology. ${ }^{6}$ TRAIL is expressed by the cells of the smooth muscle layer of the aorta and pulmonary artery and may be a survival and proliferation factor for endothelial cells (ECs). ${ }^{6}$ Expression of all the different TRAIL receptors by ECs from different vascular regions has also been reported. Regulation of bone remodeling by OPG acting through the receptor activator for nuclear factor $\mathrm{kB}$ (RANK)/receptor activator for nuclear factor $\boldsymbol{\kappa}$ ligand (RANKL) system is well established, but there is also increasing evidence supporting a role for OPG in promoting EC survival. OPG has been shown previously to colocalize with von Willebrand factor $(\mathrm{vWF})^{7}$ in Weibel-Palade bodies, the contents of which are released in response to inflammatory cytokines during vascular injury. Additionally, high expression of OPG by tumour ECs in breast cancer has been shown to correlate with a high tumour grade. ${ }^{1}$ In this study, we investigated whether TRAIL, its receptors, decoy receptors including OPG and/or RANKL are expressed in infantile haemangioma (IH), a tumour of the microvasculature ${ }^{8-10}$ that involutes spontaneously during early childhood. Proliferating IHs are composed of small densely packed capillaries lined by plump mitotically active ECs. During involution the ECs flatten the microvessel lumen dilates, and there is a progressive deposition of fibro-fatty tissue in the insterstitium. ${ }^{8-11}$

\section{Materials and methods}

\section{IH SPECIMENS}

Infantile haemangioma specimens were obtained according to a protocol approved by the Wellington Regional Ethics Committee. All lesions were categorized clinically into proliferating, involuting and involuted phases, confirmed by histological analysis as described previously. $^{8,11}$
ISOLATION AND CULTURE OF HAEMANGIOMA

\section{EXPLANT-DERIVED CELLS}

One-millimeter ${ }^{3}$ proliferating $\mathrm{IH}$ tissue explants were embedded into a single fibrin gel layer (1-mm depth) and cultured as described previously ${ }^{12}$ in the presence of $10 \%$ fetal calf serum (FCS; Invitrogen, Auckland, New Zealand). Tissue pieces were cultured for 10 days then removed carefully and discarded. The fibrin gel was digested in serum-free RPMI-1640 media (Invitrogen) containing $1 \mathrm{mg} / \mathrm{ml}$ elastase (Worthington Biochemicals Corporation, Lakewood, NJ, USA) at $37^{\circ} \mathrm{C}$ for $2-5 \mathrm{~min}$ or until the gels had dissolved. Enzyme activity was quenched with cell culture media (RPMI-1640 media containing 10\% FCS, $100 \mu \mathrm{g} / \mathrm{ml}$ streptomycin sulphate and $100 \mu \mathrm{g} / \mathrm{ml}$ penicillin $\mathrm{G}$; Invitrogen) to obtain a final concentration of $10 \% \mathrm{FCS}$. The haemangioma explant-derived cells (HaemEDCs) were recovered by centrifugation and washed prior to plating at $1 \times 10^{4}$ cells $/ \mathrm{cm}^{2}$ in cell culture media.

\section{IMMUNOHISTOCHEMISTR Y}

Immunohistochemical (IHC) staining was performed on paraffin-embedded sections of IHs. The sections were deparaffinized using standard methods and antigens retrieved by immersing slides in boiling $10 \mathrm{~mm}$ citrate buffer $\mathrm{pH} 6.0$ for $10 \mathrm{~min}$. Autofluorescence was quenched with $0.5 \%(\mathrm{w} / \mathrm{v})$ sodium borohydride, and slides blocked with $5 \%$ bovine serum albumin (BSA) prepared in $20 \mathrm{~mm}$ Tris- $\mathrm{HCl}$ buffer $\mathrm{pH} 7.6$ containing $135 \mathrm{~mm} \mathrm{NaCl}$ and $0.1 \%(\mathrm{v} / \mathrm{v})$ Tween 20 (TBST) for $1 \mathrm{~h}$. Slides were incubated overnight at $4^{\circ} \mathrm{C}$ with primary antibodies diluted appropriately (OPG, $5 \mathrm{mg} / \mathrm{ml}$; R\&D Systems, Minneapolis, MN, USA; vWF, 1:100; Dako Corporation, Glostrup, Denmark; DR4, 1:100; Imgenex, San Diego, CA, USA; DR 5, 1:200; Imgenex; DcR1, 1:100; Imgenex; DcR2, 1:200; Imgenex; RANKL, 1:200; Abcam, Cambridge, MA, USA). Bound antibodies were detected with an appropriate species-specific secondary antibody conjugated to either AlexaFluor 488 (Invitrogen) green staining or digoxigenin (DIG; Chemicon International, Temecula, CA, USA) followed by an anti-DIG rhodamine conjugate (Roche Diagnostics, Auckland, New Zealand) red staining. Cell nuclei were counterstained with either $20 \mu \mathrm{g} / \mathrm{ml}$ 4'6-diamino-2-phenylindole (DAPI; Sigma Aldrich, Auckland, New Zealand) or $10 \mu \mathrm{g} / \mathrm{ml}$ propidium iodide (PI; Sigma) and mounted in Antifade (Vectorshield, Burlingame, CA, USA). Staining of HaemEDCs plated on 13-mm diameter borosilicate glass coverslips (Biolab, Auckland, New Zealand) was performed as for paraffin sections, except 
that the cells were fixed in a mixture of $50 \%$ methanol: $50 \%$ acetone for $10 \mathrm{~min}$ at $-20^{\circ} \mathrm{C}$ and washed three times with TBST and the borohydride treatment was not performed.

RNA EXTRACTION AND QUANTITATIVE REVERSE TRANSCRIPTASE-POLYMERASE CHAIN REACTION (RT-PCR)

All reagents were purchased from Invitrogen unless specified otherwise. RNA was isolated from snap-frozen $\mathrm{IH}$ tissue (approximately $1-2 \mathrm{mg}$ ) or $1 \times 10^{6}$ HaemEDC $\left(1 \times 10^{6}\right.$ cells $)$ using Trizol according to the manufacturer's instructions until the phase separation step, following which the aqueous phase was mixed with an equal volume of $70 \%$ ethanol, applied to a high pure tissue extraction column (Roche Diagnostics) and eluted according to the manufacturer's instructions. Total RNA (approximately 200-500 ng) was reversetranscribed using Superscript III and oligo-dT $(2.5 \mu \mathrm{M})$ as primer. Amplification was performed in an $\mathrm{iQ} \mathrm{qPCR}$ system (Bio-Rad Laboratories, Hercules, CA, USA) using $10 \%$ of the reverse transcription mix, platinum SYBR green qPCR supermix-UDG, gene-specific primers $(0.1 \mu \mathrm{M})$ and fluorescein $(10 \mathrm{nM})$ in a final volume of $20 \mu \mathrm{l}$. The threshold-cycle $(\mathrm{Ct})$ was detected using the software and adjusted manually as appropriate. All reactions were performed in duplicate and controls included a no-template (water) control, as well as amplification of an equivalent amount of untranscribed RNA. Amplification was achieved with an initial denaturation at $95^{\circ} \mathrm{C}$ for $30 \mathrm{~s}$, followed by 35 cycles of denaturation at $95^{\circ} \mathrm{C}$ for $15 \mathrm{~s}$, annealing at $60^{\circ} \mathrm{C}$ for $20 \mathrm{~s}$ and extensions at $72^{\circ} \mathrm{C}$ for $20 \mathrm{~s}$. Melt-curve analysis and agarose gel electrophoresis was performed to confirm the absence of spurious amplification products. All primer sequences used are listed in Supplementary Table S1. Expression levels of mRNA are relative to glyceraldehyde-3-phosphate dehydrogenase (GAPDH) and are expressed as $\triangle \mathrm{Ct}$ values. Changes in gene expression between $\mathrm{IH}$ tissue samples and HaemEDCs are expressed as $\Delta \Delta C t$ values \pm standard error of the mean (SEM).

\section{WESTERN BLOTTING}

Total protein was extracted from HaemEDCs and $\mathrm{IH}$ tissues by solubilizing in lysis buffer $(50 \mathrm{~mm}$ Tris $-\mathrm{HCl}$ $\mathrm{pH} 6.8$ containing $5 \mathrm{~mm}$ ethylenediamine tetraacetic acid (EDTA), $2.5 \mathrm{~mm} \mathrm{NaF,} 2 \mathrm{~mm} \mathrm{NaO}_{3} \mathrm{~V}, 2 \mathrm{~mm}$ phenylmethylsulphonyl fluoride (PMSF) and 2\% sodium dodecyl sulphate (SDS) and boiling for $10 \mathrm{~min}$, followed by centrifugation to clear insoluble material. The conditioned media used to maintain HaemEDCs in culture for $96 \mathrm{~h}$ was centrifuged to remove particulate matter, concentrated approximately 50-fold using a centricon-20 (Millipore Pty Ltd, North Ryde, Australia) and then precipitated using the ProteoExtract protein precipitation kit (Chemicon International) as per the manufacturer's instructions. Protein concentration was determined using the bicinchoninic acid protein assay kit (Pierce Biotechnology Inc., Rockford, IL, USA). Total protein ( $40 \mu \mathrm{g}$ from HaemEDCs conditioned media, $100 \mu \mathrm{g}$ from HaemEDCs and $100 \mu \mathrm{g}$ from IH tissue) was combined with loading buffer [50 mm Tris $\mathrm{pH} 6.8,2 \%(\mathrm{w} / \mathrm{v})$ SDS, $0.1 \%(\mathrm{w} / \mathrm{v})$ bromophenol blue, $10 \%(\mathrm{v} / \mathrm{v})$ glycerol, $10 \% \beta$-mercapoethanol] and boiled at $100^{\circ} \mathrm{C}$ for $10 \mathrm{~min}$. Samples were subjected to electrophoresis on $1.5-\mathrm{mm}$ thick, $8 \%$ SDS polyacrylamide gels using a $4 \%$ stacking gel alongside $7 \mu \mathrm{l}$ of prestained molecular weight markers (Invitrogen) in SDS running buffer $(0.3 \%$ Tris $-\mathrm{HCl}$, $1.4 \%$ glycine, $0.1 \%$ SDS, $\mathrm{pH} 8.3$ ) at $100 \mathrm{~V}$ for $2 \mathrm{~h}$. Samples were also boiled with Nupage lithium dodecyl sulphate (LDS) sample buffer (Invitrogen) at $70^{\circ} \mathrm{C}$ for $10 \mathrm{~min}$ prior to electrophoresis through precast $4-12 \%$ gradient MES NuPAGE ${ }^{\mathrm{TM}}$ Bis-Tris gels (Invitrogen) according to the manufacturer's instructions. Protein from all samples were then transferred onto Immobilon low fluorescence polyvinylidene fluoride (PVDF) membranes (Millipore Pty Ltd) using a Trans Blot $^{\left({ }^{*}\right.}$ cell (BioRad Laboratories) for $4 \mathrm{~h}$ at $100 \mathrm{~V}$ in Western transfer buffer $(0.3 \%$ Tris- $\mathrm{HCl}, 1.4 \%$ glycine, $0.38 \%$ SDS, pH 8.3 containing 15\% methanol) and blocked with 5\% BSA in TBST for $1 \mathrm{~h}$ at room temperature. Immunoblots were incubated overnight at $4^{\circ} \mathrm{C}$ with antimouse OPG $(5 \mu \mathrm{g} / \mathrm{ml})$ and anti-mouse GAPDH (1:1000; Abcam Ltd, Cambridge, MA, USA) antibodies. Membranes were washed in TBS and bound antibodies were detected by incubation with an antimouse AlexaFluor 488 conjugate for $2 \mathrm{~h}$ at room temperature. Membranes were then washed three times in TBST and scanned using a Fujifilm FLA5100 imaging system (Fuji Photo Film Co Ltd., Tokyo, Japan). Image analysis was performed using the Image J software (National Institute of Health, Bethesda, MD, USA).

\section{STATISTICAL ANALYSIS}

Statistical analysis for qRT-PCR was performed using the R package (R Development Core Team, 2005). The linear mixed effects method of analysis was used to allow for repeated measures on subjects, ${ }^{13}$ and for each transcript a sequential Bonferroni adjustment was made for multiple testing. To test for changes in mRNA 
expression between HaemEDCs and $\mathrm{IH}$ biopsies, the non-parametric Friedmann's test for paired samples was applied to the $\Delta \Delta \mathrm{Ct}$ values obtained for each gene.

\section{Results}

IMMUNOHISTOCHEMICAL DETECTION OF TRAIL RECEPTORS IN PROLIFERATING IH

Figure 1 shows typical IHC staining of paraffin-embedded sections of proliferating IHs for TRAILRs. Immunoreactivity (IR) for DR4 (Figure 1A, red), DR5 (Figure 1B, green), DcR1 (Figure 1C, green), DcR2 (Figure 1D, green) and OPG (Figure 1E, green) was observed predominantly within the cytoplasm of the immature ECs that line the capillaries. The endothelium of $\mathrm{IH}$ showed minimal expression of RANKL compared with a small population of RANKL-positive cells (Figure 1F, red staining and white arrows) located within the interstitium between microvessels.

OPG IS EXPRESSED ONLY BY THE ENDOTHELIUM OF IMMATURE CAPILLARIES OF IH

Figure 2 shows $\mathrm{IHC}$ staining for $\mathrm{VWF}$ and $\mathrm{OPG}$ in representative proliferating (Figure $2 \mathrm{~A}-\mathrm{C}$ ) and involuting (Figure 2D-F) IH lesions. Staining for $\mathrm{vWF}$ (red) was punctate and was restricted to the plump ECs lining the immature microvessels (Figure 2A, red staining, yellow arrowheads). These same cells showed intense IR for OPG that was restricted to the endothelium (Figure 2B, green). Figure 2C contains the overlayed images. Figure $2 \mathrm{D}-\mathrm{F}$ shows staining of an involuting $\mathrm{IH}$ for $\mathrm{VWF}$ (Figure 2D, red), OPG (Figure 2E, green) and the overlayed image (Figure 2F), respectively. In contrast to the proliferating lesions
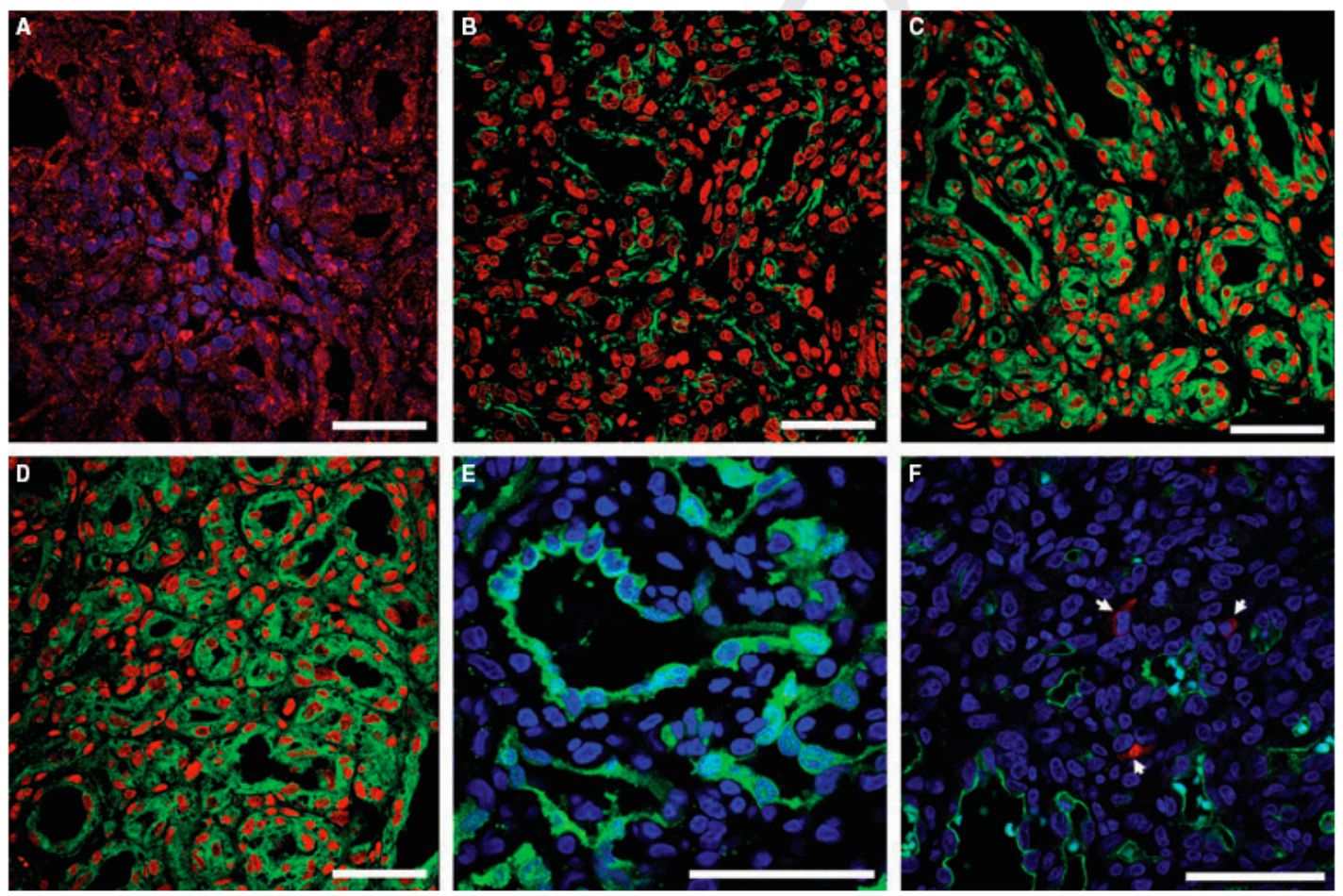

Figure 1. Confocal immunohistochemical cytoplasmic localization of tumour necrosis factor-related apoptosis-inducing ligand (TRAIL) receptors in infantile haemangioma (IH). Paraffin-embedded proliferating IH tissue sections were stained with primary antibodies against death receptor (DR)4 (red staining, A), DR5 (green staining, B), DcR1 (green staining, C), DcR2 (green staining, D) and osteoprotegerin (OPG) (green staining, E). F, Double-immunolabelling with CD34 (green) to identify the endothelium of vessels, and receptor activator for nuclear factor $\mathbf{\kappa} B$ ligand (RANKL) (red staining). A small population of RANKI-positive cells located within the interstitium between microvessels are present (white arrowheads). Cell nuclei were counter-stained with either 4'6-diamino-2-phenylindole (DAPI) (blue, A,E,F) or propidium iodide (PI) (red, A-D). Scale bars: $50 \mu \mathrm{m}$. 

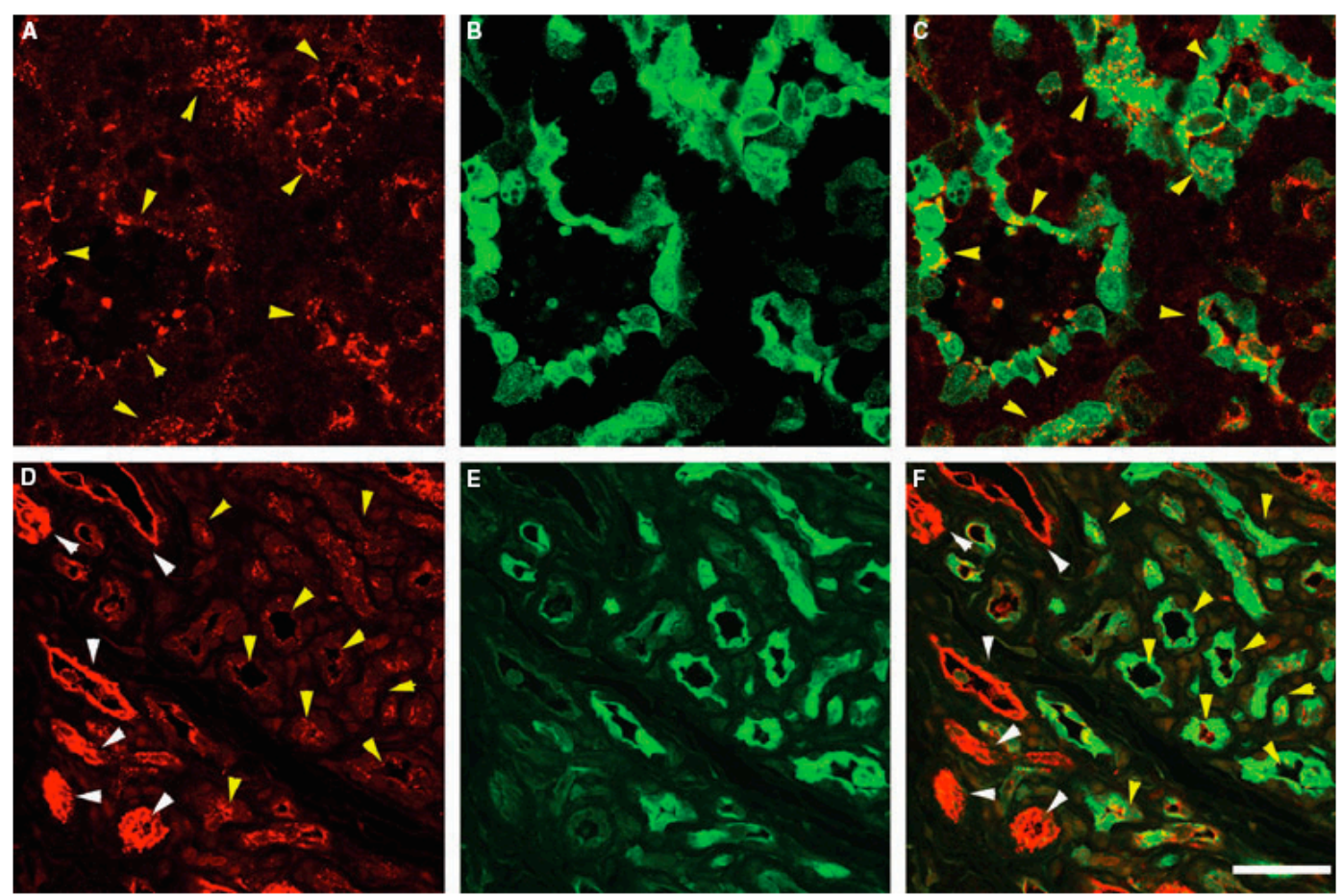

Figure 2. Immunohistochemical (IHC) staining for von Willebrand factor (vWF) and osteoprotegerin (OPG) in representative proliferating (A-C) and involuting (D-F) infantile haemangioma (IH) lesions. A, staining for vWF (red) was punctate and was restricted to the plump endothelial cells (ECs) lining the immature microvessels (yellow arrowheads). B, These same cells showed intense immunoreactivity (IR) for osteoprotegerin (OPG) (green) that was restricted to the endothelium. C. The overlayed images presented in A and B. D, IR for vWF (red): E, OPG (green) in a representative involuting IH. The lesion contains mature microvessels with dilated lumen that stained strongly for vWP (white arrowheads) as well as immature microvessels with punctate vWF staining (yellow arrowheads). F, Overlayed images presented in $\mathrm{D}$ and $\mathrm{E}$ and shows clearly that the immature capillaries with punctate vWF staining predominantly express OPG.

(Figure $2 \mathrm{~A}-\mathrm{C}$ ), mature microvessels in Figure $2 \mathrm{D}-\mathrm{F}$ with dilated lumen stained strongly for vWF (white arrowheads) as well as immature microvessels with punctate vWF staining (yellow arrowheads). Figure $2 \mathrm{~F}$ shows the overlayed images of the VWF and OPG staining, and illustrates clearly that only the immature capillaries with punctate vWF staining, expressed OPG (Figure 2E, green).

IMMUNOSTAINING OF HAEMEDCS FOR OPG AND OTHER TRAIL RECEPTORS

To determine if the staining observed on paraffinembedded IH sections was recapitulated on HaemEDCs, staining for OPG and other TRALRs was performed on primary cultures of HaemEDCs fixed on coverslips. Figure 3 shows strong IR (green) for DR4 (Figure 3A), DR5 (Figure 3B), DcR1 (Figure 3C) and DcR2

\footnotetext{
O 2011 Blackwell Publishing Ltd, Histopathdogy
}

(Figure 3D) of HaemEDCs, corresponding with the IHC staining of these receptors in IH tissue sections presented in Figure 1A-D. Figure 3E shows that OPG (green staining) was secreted by HaemEDCs when grown in culture. Staining of HeLa cells as a positive control for OPG (green) is shown in Figure 3F.

\section{EXPRESSION OF MRNA FOR TRAIL AND TRAIL} RECEPTORS AND RANKL IN IH TISSUE

To confirm the expression of TRAIL and its receptors as determined by immunohistochemistry, qRT-PCR analysis was performed on RNA extracted from proliferating and involuting $\mathrm{IH}$ tissues. Preliminary experiments determined that GAPDH was the most suitable internal reference gene to use in qRT-PCR of those we tested (18SrRNA, hypoxanthine ribotransferase and cyclophilin A), as its expression did not 

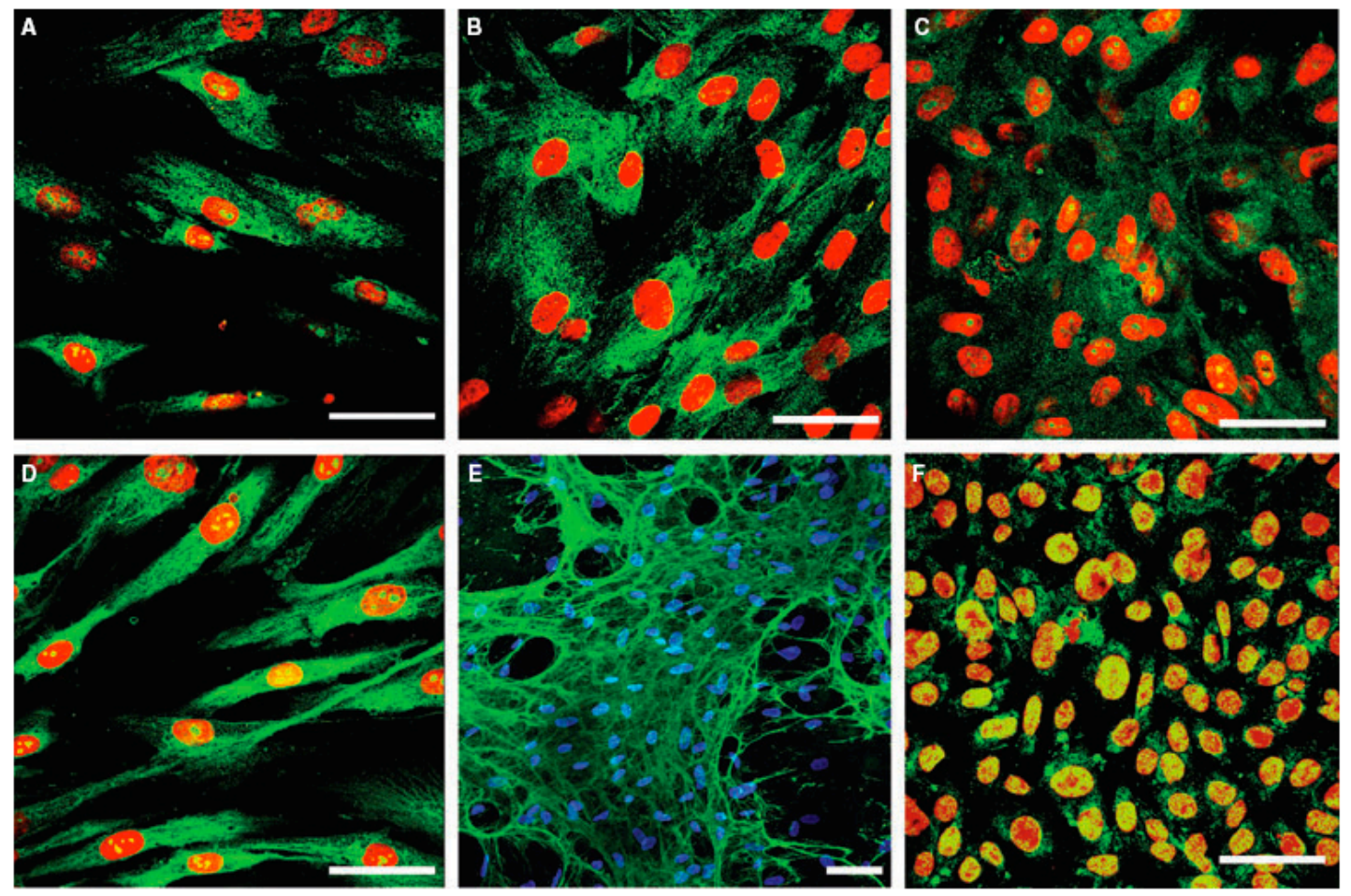

Figure 3. Confocal immunohistochemical localization of tumour necrosis factor-related apoptosis-inducing ligand (TRAIL) receptors on haemangioma explant-derived cells (HaemEDCs). Cells were fixed and stained with antibodies against death receptor (DR)4 (green staining, A), DR5 (green staining, B), DcR1 (green staining, C) and DcR2 (green staining, D). E, Osteoprotegerin (OPG) is secreted (green staining) by HaemEDCs. F, Staining of HeLa cells as a positive control for OPG (green). Cell nuclei were counterstained with propidium iodide (PI) (A-D, F, red) or 4'6-diamino-2-phenylindole (DAPI) (E, blue). Scale bars: $50 \mu \mathrm{m}$.

vary significantly between the proliferating and involuting phases. The mean $\mathrm{Ct}$ value for GAPDH was 18 $(\mathrm{SEM}=1.0)$ when the equivalent of $50 \mathrm{ng}$ of RNA was amplified. The $\mathrm{Ct}$ values for 11 proliferating, four involuting and six involuted $\mathrm{IH}$ samples are presented in Table 1. The expression profile for TRAIL and the TRAILRs was similar for the proliferating, involuting and involuted samples, with all mRNAs being slightly more abundant in the proliferating samples. DR4 mRNA was most abundant and was expressed at greater levels than DR5. The decoy receptors, DcR1, Dcr2 and OPG, were detected at lower but comparable levels.

EXPRESSION MRNA FOR TRAIL AND TRAIL RECEPTORS IN HAEMEDCS

qRT-PCR was used to compare the mRNA expression profiles for TRAIL and its receptors, DR4, DR 5, DcR1, DcR2, and OPG in HaemEDCs with the original tissue samples from which the HaemEDCs were isolated. Relative expression to GAPDH is presented as $\Delta \mathrm{Ct}$ values in Table 2 . The difference in mRNA expression between the HaemEDCs and matched original tissues is shown graphically as the average $\Delta \Delta \mathrm{Ct}$ values for six biopsies analysed in Figure 4A. Small differences in expression of mRNA for DR4, DR 5 and DcR1 were found, but there was a large induction of OPG expression $(P<0.001)$ and DcR2 $(P<0.01)$ in the HaemEDCs. This increase in decoy receptor expression was also matched by a similar decrease in TRAIL expression $(P<0.001)$ by the HaemEDCs.

\section{HAEMEDCS SECRETE OPG}

In proliferating IH tissue sections, OPG expression (Figure 1E, green staining) was detected predominantly in the cytoplasm. However, when HaemEDCs were grown in culture for 7 days extracellular OPG (Figure $5 \mathrm{~B}$, green) was detected at significantly higher levels 
Table 1. Expression of tumour necrosis factor-related apoptosis-inducing ligand (TRAIL) and TRAIL receptors mRNA in infantile haemangioma

\begin{tabular}{lllllll}
\hline Phase & TRAIL & DR4 & DR5 & DcR1 & DcR2 & OPG \\
\hline Proliferating $(n=11)$ & $4.3 \pm 0.5$ & $8.1 \pm 0.2$ & $14.3 \pm 0.7$ & $9.5 \pm 0.7$ & $9.5 \pm 0.3$ & $10.7 \pm 0.5$ \\
\hline Involuting $(n=4)$ & $5.2 \pm 0.3$ & $9.9 \pm 0.3$ & $>18$ & $13.6 \pm 0.3$ & $12.1 \pm 0.4$ & $11.7 \pm 0.7$ \\
\hline Involuted $(n=6)$ & $3.4 \pm 1.4$ & $8.3 \pm 3.4$ & $13.6 \pm 5.6$ & $10.2 \pm 4.2$ & $10 \pm 4.1$ & $8.8 \pm 3.6$ \\
\hline
\end{tabular}

Relative expression levels of mRNA for TRAIL and TRAIL receptors were determined in 11 proliferating, four involuting and six involuted IH specimens by quantitative reverse transcription-polymerase chain reaction (qRT-PCR) using glyceraldehyde-3phosphate dehydrogenase (GAPDH) as the internal house-keeping reference gene.

Values are expressed as the average $\Delta$ Ct value obtained for each gene \pm standard error of the mean.

Lesions from all phases expressed high levels of TRAIL mRNA and moderate levels of death receptor (DR)4, DcR1, DcR2 and osteoprotegerin (OPG) mRNA.

Low levels of DR5 mRNA were detected in all samples.

Table 2. Differences in mRNA levels for tumour necrosis factor-related apoptosis-inducing ligand (TRAIL) and TRAIL receptors in proliferating infantile haemangioma $(\mathrm{IH})$ tissue and haemangioma explant-derived cells (HaemEDCs)

\begin{tabular}{lcccccc}
\hline & TRAIL & DR4 & DR5 & DcR1 & DcR2 & OPG \\
\hline IH tissue & 5.1 & 7.6 & 12.6 & 8.4 & 8.7 & 10.7 \\
\hline HaemEDCs & 15.9 & 11.3 & 14.7 & 10.9 & 5.7 & 4.3 \\
\hline
\end{tabular}

OPG, Osteoprotegerin; DR, death receptor.

mRNA levels for TRAIL and TRAIL receptors were determined in six proliferating IH biopsies and the corresponding HaemEDC isolates from each tissue sample.

The table gives the average $\triangle \mathrm{Ct}$ values obtained for each gene from tissue pieces (IH tissue) and cell isolates (HaemEDCs).

compared with that within the cytoplasm of cells (Figure 3E).

To confirm that the HaemEDCs secrete and release OPG when cultured in vitro, Western blot analysis (Figure 4B) was performed on culture media (M) in which HaemEDCs had been cultured, as well as the HaemDC cell lysates (CLs) and tissue lysates (TLs). IR bands corresponding to monomeric OPG of Mr $60 \mathrm{kDa}$ (Figure $4 \mathrm{~A}$, lane $\mathrm{M},{ }^{*}$ ) and an oligomeric glycosylated form of Mr $220 \mathrm{kDa}$ (Figure 4A, lane M, black arrow) was detected in the culture medium, consistent with previous reports. ${ }^{14}$ No immunoreactive bands were detected in unconditioned media analysed in the same manner (data not shown). Analysis of $100 \mu \mathrm{g}$ of total protein extracted from HaemEDCs and proliferating $\mathrm{IH}$ tissue showed that OPG protein expression correlated with the mRNA study, in that higher levels of OPG were detected in HaemEDCs compared with proliferating $\mathrm{IH}$ tissues. Figure 4B shows that monomeric OPG was detected in HaemEDCs CLs, but not in IH TLs. The second immunoreactive band at $220 \mathrm{kDa}$ identified in culture media (M, black arrow) was also detected in HaemEDCs (lane CL, black arrow) but not in extracts from proliferating IH tissue (lane TL, black arrow).
Immunoblotting for GAPDH (Figure 4B, lower panel) showed that GAPDH was not detected in the media but was detected in the TLs and CLs, confirming that the OPG in media was secreted and did not arise from lysed cells in the culture dish.

\section{Discussion}

There is now a significant body of literature that demonstrates a role for OPG in the regulation of $\mathrm{EC}$ function, in bone remodelling, and also as a regulator of TRAIL-induced apoptosis. ${ }^{3}$ In this study, we show that proliferating $\mathrm{IH}$ expresses all five TRAIL receptors including $\mathrm{OPG}$, and that $\mathrm{OPG}$ is expressed by the immature mitotically active ECs that line the capillaries. We have shown recently that the immature ECs that form the capillary endothelium in proliferating $\mathrm{IH}$ express primitive markers and transcription factors consistent with a haemogenic endothelium. ${ }^{15}$ Here we confirm the immature nature of the endothelium by showing that that mature capillaries with wide lumen express vWF factor strongly, as expected, whereas the immature capillaries with narrow lumen show weaker punctate vWF staining ${ }^{10,15,16}$ (Figure 1). 

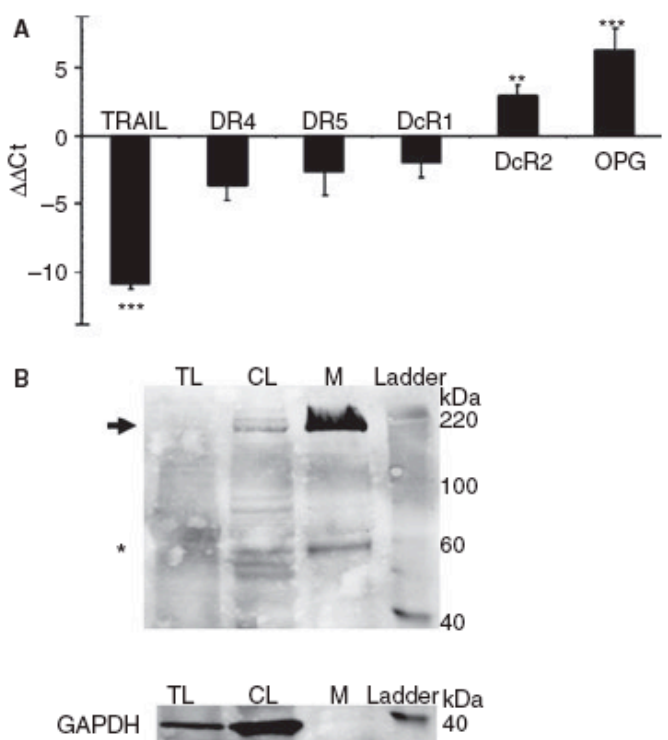

Figure 4. Expression of tumour necrosis factor-related apoptosisinducing ligand (TRAIL), TR AIL receptors (TRAILRs) and osteoprotegerin $(\mathrm{OPG})$ in infantile haemangioma (IH) tissues and HaemEDCs. A, Relative expression of TRAIL, TRAILRs and osteopmotegerin (OPG) mRNA from six haemangioma explant-derived cell (HemEDC) isolates. Relative expression was determined by quantitative reverse transcription-polymerase chain reaction ( $\mathrm{qRT}-\mathrm{PCR}$ ) on mRNA extracted from matched proliferating IH tissue and the biopsy from which the cells were isolated. Expression was normalized to that for glyceraldehyde-3phosphate dehydrogenase (GAPDH) and calculated as a $\Delta \mathrm{Ct}$. Differences between cell lines relative to tissues were determined as $\Delta \Delta \mathrm{Ct}$ values. Data presented show the average $\triangle \Delta C t$ value obtained for each gene between tissue and cells. A low value indicates higher expression of the gene in the tissue, while a high value shows higher mRNA expression of that gene by HaemEDCs. Significant differences in gene expression between IH tissues and cells were detected using Friedmann's non-parametric test for paired samples, where ** represents significance at $P<0.01$ and $* * *$ at $P<0.001$. B. Immunoblot for $\mathrm{OPG}$ from a proliferating IH tissue lysate (TL), a cell lysate (CL) from cell matched to the tissue, and the conditioned culture medium $(M)$ in which the cells were grown. Approximately $100 \mu$ g of total protein was loaded in each lane. The Mr for monomeric OPG is indicated by ${ }^{*}$, and that for the oligomeric glycosylated is shown by the black arrow. The lower panel shows the results of immunoblotting for GAPDH and confirms that the OPG present in the media was secreted and did not arise from dead lysed cells.

Our finding that $O P G$ is expressed strongly by the immature cells of the vessel endothelium in $\mathrm{IH}$ is consistent with the growing body of evidence for a role for OPG in tumour cell biology and EC function. ${ }^{14,17,18}$ A functional role for OPG in EC is supported further by the demonstration of physical association of OPG and vWF in Weibel-Palade bodies of ECs, ${ }^{19}$ and also in platelets. ${ }^{20}$ The inverse relationship between the expression of $\mathrm{OPG}$ and the expression of $\mathrm{vWF}$ that we show (Figure 1) supports an anti-apoptotic and proangiogenic role for OPG in $\mathrm{IH}$. This assertion is in agreement with the study of Kobayashi-Sakamoto et al. ${ }^{18}$ showing that OPG protects ECs from undergoing apoptosis and detachment as well as promoting angiogenesis via Src signalling. The anti-apoptotic effects of OPG are thought to be mediated via binding of OPG to TRAIL and inhibiting TRAIL-induced apoptosis, such that OPG is a pro-tumour survival factor. ${ }^{21}$ The inverse relationship for the expression of OPG and the expression of $\mathrm{vWF}$ in proliferating IH that we report supports the notion that the ECs lining the endothelium are resistant to TRAI-induced apoptosis. As the capillaries in $\mathrm{IH}$ mature and $\mathrm{vWF}$ expression increases there is a concomitant decrease in the expression of OPG that we reason makes the ECs more sensitive to TRAIL-induced apoptosis and less sensitive to proangiogenic stimuli. This mechanism may account for the increase in apoptosis and decreased angiogenesis that take place as the $\mathrm{IH}$ tissue remodels and becomes fibro-fatty during involution.

Reid et al. ${ }^{17}$ show that OPG enhances the proangiogenic effects of VEGF and that breast cancer cells increase OPG expression and secretion via a mechanism involving ligation of the integrin $\alpha_{v} \beta_{3}$ and $\mathrm{NF \kappa B}$ activation. The report of the expression of the same integrins in the endothelium of proliferating $\mathrm{IH}^{22}$ supports a possible role for OPG and $\alpha_{v} \beta_{3}$ in the aggressive angiogenesis seen during proliferation of $\mathrm{IH}$.

We show that HaemEDCs express abundance of OPG protein that is secreted into the extracellular matrix. We also detected OPG protein in the conditioned media of HaemEDCs. Atkins et al. ${ }^{23}$ have shown a similar pattern of extracellular OPG expression in human osteoblast-like cells and correlated increased OPG expression with TRAIL resistance. There is now mounting evidence suggesting that the interaction of OPG with TRAIL is biologically important in in-vitro culture systems where OPG acts as a cell-survival factor. OPG has been shown to protect serum-starved ECs and fibroblast-like synovial cells from TRAIL-triggered cell death. ${ }^{24,25}$ Binding of OPG to TRAIL also promotes the survival of prostate cancer cells, ${ }^{2}$ breast cancer cells, ${ }^{26}$ multiple myeloma cells ${ }^{5}$ and ameloblastoma cells. ${ }^{3,27}$

It is possible that HaemEDCs express high levels of OPG as a stress response when in culture in vitro. Previous studies have shown an up-regulation of $\mathrm{OPG}$ mRNA in response to stress. ${ }^{28,29}$ High concentrations of OPG protein have also been detected in the conditioned medium of osteoblastic cells subjected to cyclic tensile strain. ${ }^{30}$ The presence of secreted OPG in the conditioned media of HaemEDCs detected in our study 
is consistent with these findings. Western blotting of $\mathrm{IH}$ tissue extracts, HaemEDCs and their conditioned media shows that the HaemEDCs express significantly more OPG protein than the tissue extracts and that the excreted high molecular weight $\mathrm{OPG}$ found in the extracellular matrix and media is most probably glycosylated, as reported previously in other systems.

While the physiological regulator of $\mathrm{OPG} / \mathrm{vWF}$ release is yet to be determined in $\mathrm{IH}$ it is interesting to note that angiotensin II, the pro-angiogenic peptide of the renin-angiotensin system (RAS), has been shown to promote secretion of OPG. ${ }^{31}$ We have also demonstrated recently a critical role for the RAS in the pathogenesis of $\mathrm{IH}^{32}$ which has led us to speculate that the angiotensin II levels that are presumed to be high during the proliferation phase of $\mathrm{IH}$, due to physiological high levels of rennin, ${ }^{32}$ may contribute to increased secretion of OPG and thus preventing apoptosis seen during proliferation.

Osteoprotegerin is also a ligand for RANKL, and acts to inhibit the effects of RANKL on its receptor RANK. ${ }^{21}$ Given that the expression of RANKL is restricted to cells of the interstitium away from the endothelial OPGproducing cells, we infer that $\mathrm{OPG}$ acts via the TRAIL but not the RANKL/RANK pathway during IH proliferation. However, the role of the OPG and the RANKL/ RANK in IH progression warrants further investigation.

This novel observation of the involvement of the OPG/TRAIL pathway in proliferating IH infers a critical role for this anti-apoptotic pathway during proliferation, and may account for the accumulation of cells seen during the proliferating phase of $\mathrm{IH}$.

\section{Declarations of interest}

The authors do not have any conflict of interest.

\section{Acknowledgements}

This work is supported by grants from the Wellington Regional Plastic Surgery Unit Research and Education Trust: the Wellington Medical Research Foundation: the Surgical Research Trust, Pub Charities and the Cancer Society of New Zealand. Dr Vishvanath was supported, in part, by the Cancer Society of New Zealand. Dr Itinteang is supported by the Royal Australasian College of Surgeons' Foundation for Surgery Scholarship.

\section{References}

1. Cross SS, Yang Z, Brown NJ et al. Osteoprotegerin (OPG) - a potential new role in the regulation of endothelial cell phenotype and tumour angiogenesis? Int. J. Canor 2006; 118; 1901-1908.

2. Holen I, Croucher PI, Hamdy FC, Eaton CL. Osteoprotegerin $(\mathrm{OPG})$ is a survival factor for human prostate cancer cells. Cancer Res. 2002; 62; 1619-1623.

3. Reid P. Holen I. Pathophysiological roles of osteoprotegerin (OPG). Eur. J. Cell Biol. 2009; 88; 1-17.

4. Kischkel FC, Lawrence DA, Chuntharapai A, Schow P, Kim KJ. Ashkenazi A. Apo2L/TRAII-dependent recruitment of endogenous FADD and caspase- 8 to death receptors 4 and 5. Immunity 2000; 12; 611-620.

5. Shipman CM, Croucher PI. Osteoprotegerin is a soluble decoy receptor for tumour necrosis factor-related apoptosis-inducing ligand/Apo2 ligand and can function as a paracrine survival factor for human myeloma cells. Cancer Res. 2003; 63; 912-916.

6. Van Campenhout A, Golledge J. Osteoprotegerin, vascular calcification and atherosclerosis. Atherosclerosis 2009; 204; 321-329.

7. Zannettino A, Holding C. Diamond P et al. Osteoprotegerin (OPG) is localised to the Weibel-Palade bodies of human vascular endothelial cells and is physically associated with von Willebrand factor. J. Cell. Physiol. 2005; 204; 714-723.

8. Tan ST, Velickovic M, Ruger BM, Davis PP. Cellular and extracellular markers of hemangioma. Plast. Reconstr. Surg. 2000; 106; 529-537.

9. Tan ST, Itinteang T, Leadbitter P. Low-dose propranolol for infantile haemangioma. J. Plast. Reconstr. Aesthet. Surg. 2010; ???? ????-????. doi: 10.1016/j.bjps. 2010.06.010.

10. Itinteang T, Tan ST, Brasch H, Day DJ. Primitive mesodermal cells with a neural crest stem cell phenotype predominate proliferating infantile haemangioma. J. Clin. Pathol. 2010; 63; $771-776$.

11. Tan ST, Wallis RA, He Y, Davis PF. Mast cells and hemangioma. Plast. Reconstr. Surg. 2004: 113; 999-1011.

12. Tan ST, Hasan Q, Velickovic M, Ruger BM, Davis RPF, Davis PF. A novel in vitro human model of hemangioma. Mod. Pathol. 2000; 13; 92-99.

13. Pinheiro JC, Bates DM. Statistics and computing series. NY: Springer-Verlag, 2000.

14. Zauli G, Melloni E, Capitani S, Secchiero P. Role of full-length osteoprotegerin in tumour cell biology. Cell. Mol. Life Sci. 2009; 66; $841-851$.

15. Itinteang T, Tan ST, Brasch H, Day DJ. Haemogenic endothelium in infantile haemangioma. J. Clin. Pathol. 2010; 63; 982-986.

16. Hatzopoulos AK, Folkman J, Vasile E, Eiselen GK, Rosenberg RD. Isolation and characterization of endothelial progenitor cells from mouse embryos. Development 1998; 125; 1457-1468.

17. Reid P, Brown N, Holen I. Breast cancer cells stimulate osteoprotegerin $(\mathrm{OPG})$ production by endothelial cells through direct cell contact. Mol. Cancer 2009; 8; 49 .

18. Kobayashi-Sakamoto M, Isogai E, Holen I. Osteoprotegerin induces cytoskeletal reorganization and activates $\mathrm{FAK}, \mathrm{Src}$, and ERK signalling in endothelial cells. Eur. J. Haematol. 2010; 85; 26-35.

19. Baud'huin M, Duplomb L, Teletchea S et al. Factor VIII-von Willebrand factor complex inhibits osteoclastogenesis and controls cell survival. J. Biol. Chem. 2009; 284; 31704-31713.

20. Chollet ME, Brouland JP, Bal dit Sollier C, Bauduer F, Drouet L, Bellucci S. Evidence of a colocalisation of osteoprotegerin (OPG) with von Willebrand factor (VWF) in platelets and megakaryocytes alpha granules. Studies from normal and grey platelets. $\mathrm{Br}$. J. Haematol. 2010; 148; 805-807.

21. Fili S, Karalaki M, Schaller B. Therapeutic implications of osteoprotegerin. Canor Cell Int. 2009; 9; 26. 
22. Ritter MR, Dorrell MI, Edmonds J, Friedlander SF, Friedlander M. Insulin-like growth factor 2 and potential regulators of heman gioma growth and involution identified by large-scale expression analysis. Proc. Natl Acad. Sa. USA 2002; 99; 7455-7460.

23. Atkins GJ, Bouralexis $\mathrm{S}$, Haynes DR et al. Osteoprotegerin inhibits osteoclast formation and bone resorbing activity in giant cell tumours of bone. Bone 2001; 28; 370-377.

24. Pritzker LB, Scatena M, Giachelli CM. The role of osteoprotegerin and tumour necrosis factor-related apoptosis-inducing ligand in human microvascular endothelial cell survival. Mol. Biol. Cell 2004: 15; 2834-2841.

25. Miyashita T, Kawakami A. Nakashima T et al. Osteoprotegerin (OPG) acts as an endogenous decoy receptor in tumour necrosis factor-related apoptosis-inducing ligand (TRAIL)-mediated apop tosis of fibroblast-like synovial cells. Clin. Exp. Immunol. 2004: 137; $430-436$

26. Neville-Webbe HL, Cross NA, Eaton CL et al. Osteoprotegerin (OPG) produced by bone marrow stromal cells protects breast cancer cells from trail-induced apoptosis. Breast Cancer Res. Treat. 2004; 86; 271-282.

27. Sandra F, Hendarmin L, Nakamura S. Osteoprotegerin (OPG) binds with tumour necrosis factor-related apoptosis-inducing ligand (TRAIL): suppression of TRAIL-induced apoptosis in ameloblastomas. Oral Oncol. 2006; 42; 415-420.

28. Kanzaki H. Chiba M. Sato A et al. Cyclical tensile force on periodontal ligament cells inhibits osteoclastogenesis through OPG induction. J. Dent. Res. 2006; 85; 457-462.

29. Yamamoto Y, Udagawa N, Matsuura S et al. Osteoblasts provide a suitable microenvironment for the action of receptor activator of nuclear factor-\{kappa\}B ligand. Endocrinology 2006; 147; 3366-3374.

30. Tang L, Lin Z, Li Y-M. Effects of different magnitudes of mechanical strain on osteoblasts in vitro. Biochem. Biophys. Res. Commun. 2006; 344; 122-128.

31. Moran CS, Cullen B, Campbell JH, Golledge J. Interaction between angiotensin II, osteoprotegerin, and peroxisome proliferatoractivated receptor-I $\hat{I}^{3}$ in abdominal aortic aneurysm. J. Vasc. Res. 2009; 46; 209-217.

32. Itinteang T, Brasch HD, Tan ST, Day DJ. Expression of components of the renin-angiotensin system in proliferating infantile haemangioma may account for the propranolol-induced accelerated involution. J. Plast. Reconstr. Aesthet. Surg. 2010; ????; ????-???. doi: 10.1016/j.bjps.2010.08.039.

\section{Supporting Information}

Additional Supporting information may be found in the online version of this article:

Table S1. qRT-PCR primers used in this study.

Please note: Wiley-Blackwell are not responsible for the content or functionality of any supporting materials supplied by the authors. Any queries (other than missing material) should be directed to the corresponding author for the article. 


\section{Chapter 8: Renin-Angiotensin System and Infantile Haemangioma}

\subsection{Introduction and Overview}

Chapter 4 describes the expression of angiotensin converting enzyme (ACE) on the putative haemogenic endothelium of the capillaries of proliferating $\mathrm{IH}$. The demonstration of the expression of ACE is significant as it suggests a possible role of the renin-angiotensin system (RAS) in the biology of $\mathrm{IH}$, and a potential mechanism to account for the action of $\beta$-blockers, such as propranolol, in accelerating involution of proliferating $\mathrm{IH}$.

In this chapter I have demonstrated the expression of angiotensin receptor 2 (ATR2), but not angiotensin receptor 1 isoform within the capillaries of proliferating $\mathrm{IH}$. This is intriguing as recent studies have demonstrated critical involvement of ATR2 during primitive haematopoiesis with the angiotensin II (ATII) being the active peptide ${ }^{73}$.

Additionally, data is presented to show that the administration of ATII to cells derived from proliferating $\mathrm{IH}$ explants causes them to form blast colonies during cell culture. The blasts express similsr proteins to those seen in EPCs that have been demonstrated to be involved in $\mathrm{IH}$ progression. It is intriguing to suggest that these EPCs/blasts are indeed downstream effects of excessive ATII production.

Interestingly white, female, and premature infants who have higher incidence of $\mathrm{IH}$ also have higher levels of physiological renin compared with black, male, and full-term infants ${ }^{74-76}$. Renin is known to convert 
angiotensinogen to angiotensin $\mathrm{I}^{77}$, which is then converted to the vaso-active ATII within the tumour to act in an autocrine/paracrine fashion to promote proliferation of the EPC phenotypic haemogenic endothelium, as previously discussed in Chapter 4.

This chapter also hypothesises that IH is governed by the RAS. High levels of renin during the proliferative phase of $\mathrm{IH}$, lead to elevated levels of ATII which causes proliferation of the haemogenic endothelium.

\subsection{Contribution}

All experiments were performed by myself, the manuscript was written by myself and edited by my supervisors 
8.3. Manuscript: Expression Of Components Of The ReninAngiotensin System In Proliferating Infantile Haemangioma May Account For Propranolol May Account For Propranolol-Induced Accelerated Involution 


\title{
Expression of components of the renin-angiotensin system in proliferating infantile haemangioma may account for the propranolol-induced accelerated involution $^{\text {is }}$
}

\author{
Tinte Itinteang ${ }^{a, b, c}$, Helen D. Brasch ${ }^{b, c, d}$, Swee T. Tan ${ }^{a, b, c, e, *, f}$, \\ Darren J. Day a,b,c, \\ ${ }^{a}$ School of Biological Sciences, Victoria University of Wellington, New Zealand \\ ${ }^{\mathrm{b}}$ Gillies McIndoe Research Institute, New Zealand \\ ${ }^{c}$ Centre for the Study \& Treatment of Vascular Birthmarks, Wellington Regional Plastic, Maxillofacial \& Burns Unit, \\ New Zealand \\ ${ }^{\mathrm{d}}$ Department of Pathology, Hutt Hospital, New Zealand \\ 'University of Otago, Wellington, New Zealand
}

Received 31 March 2010; accepted 30 August 2010

\section{KEYWORDS}

Infantile haemangioma;

Propranolol;

Renin;

Angiotensin;

ACE
Summary Infantile haemangioma is a benign tumour of the microvasculature characterised by excessive proliferation of immature endothelial cells. It typically undergoes rapid proliferation during infancy followed by spontaneous slow involution during childhood often leaving a fibrofatty residuum.

In 2008, propranolol, a non-selective $\beta$-blocker, was serendipitously discovered to induce accelerated involution of a proliferating infantile haemangioma. However, the mechanism by which propranolol causes this dramatic effect is unclear. Using immunohistochemical staining,

\footnotetext{
This article was presented, in part, at the Australian and New Zealand Vascular Anomalies Interest Group Meeting, 22nd October 2009, Melbourne, Australia; the New Zealand Association of Plastic Surgeons' Annual Scientific Meeting, 21st November 2009, Auckland, New Zealand; and the Royal Australasian College of Surgeons' 46th Surgical Research Society Meeting, Adelaide, Australia, 20th November 2009; and the 8th Annual Meeting of the International Society for Stem Cell Research, San Francesco, USA, 16-19 June 2010. This article was awarded the John Mulliken Prize for the best basic science paper at the 18th International Workshop on Vascular Anomalies, Brussels, Belgium, 21-24 April 2010.

* Corresponding author. Wellington Regional Plastic, Maxillofacial \& Burns Unit, Hutt Hospital, Private Bag 31-907, High Street, Lower Hutt, Wellington, New Zealand. Tel.: +64 4 5872506; fax: +64 45872510.

E-mail address: swee.tan@huttvalleydhb.org.nz (S.T. Tan).

${ }^{f}$ Equal senior authors.
}

1748-6815/\$ - see front matter (C) 2010 British Association of Plastic, Reconstructive and Aesthetic Surgeons. Published by Elsevier Ltd. All rights reserved. doi:10.1016/j.bjps.2010.08.039 
we show that the $\mathrm{CD} 34^{+}$endothelial progenitor cells of the microvessels in proliferating infantile haemangioma express angiotensin-converting enzyme and angiotensin II receptor-2, but not angiotensin II receptor- 1 . We have also shown using our in vitro explant model that the cells emanating from proliferating haemangioma biopsies form blast-like structures that proliferate in the presence of angiotensin II. We present here a plausible model involving the renin-angiotensin system that may account for the propranolol-induced accelerated involution of proliferating infantile haemangioma.

(C) 2010 British Association of Plastic, Reconstructive and Aesthetic Surgeons. Published by Elsevier Ltd. All rights reserved.

Infantile haemangioma is a primary tumour of the microvasculature. It is the most common tumour of infancy affecting $10 \%$ of white children, with preponderance in Caucasian, female and premature infants. ${ }^{1-3}$ Infantile haemangioma is characterised by rapid proliferation during infancy (proliferative phase), followed by slow spontaneous involution (involuting phase) with continued improvement up to 12 -years of age (involuted phase). ${ }^{1,4}$

Until recently, high-dose steroid therapy was the mainstay treatment for problematic proliferating infantile haemangioma. ${ }^{5-7}$ In 2008, a French group serendipitously observed accelerated involution of a large facial proliferating haemangioma in an infant following treatment with propranolol for steroid-induced hypertrophic cardiomyopathy. ${ }^{8}$ Propranolol, a commonly used non-selective $\beta$-blocker, is now the preferred treatment for problematic proliferating infantile haemangioma. ${ }^{9-12}$ Figure $1 \mathrm{~A}-\mathrm{C}$ demonstrates our experience with this novel use of propranolol in a 3-month-old infant with a proliferating haemangioma involving the mid-face.

The mechanism of action of propranolol in proliferating infantile haemangioma is unknown ${ }^{9}$ with recent reports, suggesting a role for direct inhibition of $\beta$-adrenergic receptor. ${ }^{10}$ In this report, we propose that propranolol acts via the renin-angiotensin system (RAS) in regulating accelerated involution of proliferating infantile haemangioma by inhibiting the proliferation of CD34 $4^{+} /$VEGFR $-2^{+}$endothelial progenitor cells (EPCs).

\section{Methods}

\section{Immunohistochemistry}

From 10 patients, aged 3-10 (mean 6) months, 4- $\mu \mathrm{m}$ paraffin-embedded sections of proliferating infantile haemangioma biopsies were obtained, according to a protocol approved by the Wellington Regional Ethics Committee.

Routine rehydration and antigen retrieval procedures were performed with $10 \mathrm{mM}$ sodium citrate buffer $\mathrm{pH} 6.0$ (Sigma-Aldrich, Melbourne, Australia) and the slides were incubated overnight with primary antibodies appropriately diluted in $15 \mathrm{mM}$ Tris- $\mathrm{HCl} \mathrm{pH} \mathrm{7.6} \mathrm{containing} 136 \mathrm{mM} \mathrm{NaCl}$ and $0.01 \%$ Tween (TBST). The antibodies used were GLUT-1, 1:500; CD34, 1:300; smooth muscle actin (SMA), 1:400; VEGFR-2, 1:300; CD133，1:300; angiotensin-converting enzyme (ACE), 1:200; angiotensin receptor 1 (ATR1), 1:200; and angiotensin receptor 2 (ATR2), 1:100, all were obtained from Abcam, MA. Slides were washed and bound antibodies detected with an appropriate secondary antibody for $2 \mathrm{~h}$ (Goat anti-Mouse alexa-488 or Chicken anti-Rabbit alexa594; Invitrogen, Melbourne, Australia), with the exception of VEGFR-2 and ATR2, which were detected with an anti-rabbit digoxigenin (Roche diagnostics, Auckland, NZ) conjugate followed by an anti-digoxigenin-Rhodamine (Chemicon, Sydney, Australia). Cell nuclei were counterstained with 4', 6diamidino-2-phenylindole (DAPI) in Gold Antifade (Invitrogen) prior to cover slip mounting. Negative controls included staining of paraffin sections of tissue not expected to be immunoreactive with the same series of antibodies (human uterine fibroid), and also by omission of the primary antibodies when staining selected proliferating infantile haemangiomas. These controls showed the expected staining pattern, consistent with the previous reported specificity for the antibodies used.

\section{Response of haemangioma cell culture to angiotensin II treatment}

Fresh proliferating infantile haemangioma biopsies from three patients, aged 3,6 and 8 months, were cultured using
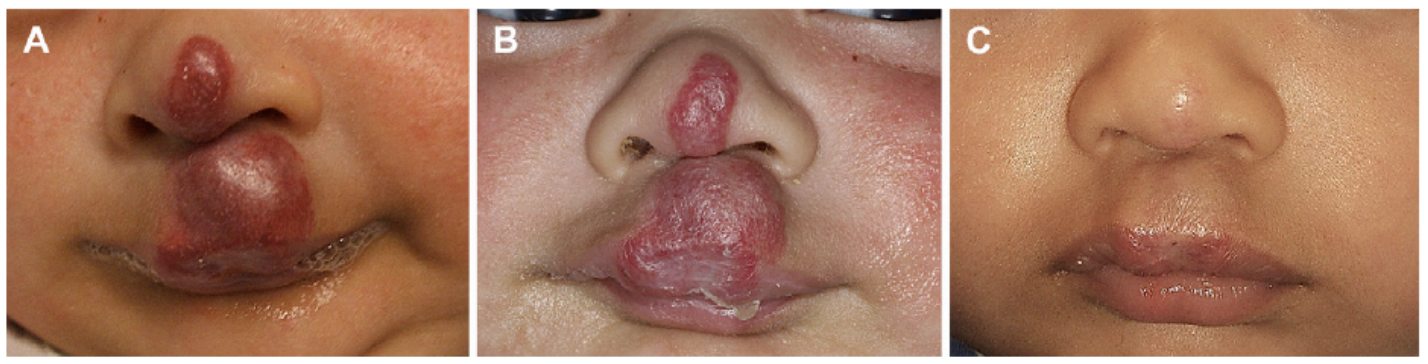

Figure 1 An infant with a proliferating haemangioma at (A) 3 months of age, (B) 3 days and (C) 3 months after propranolol treatment. 

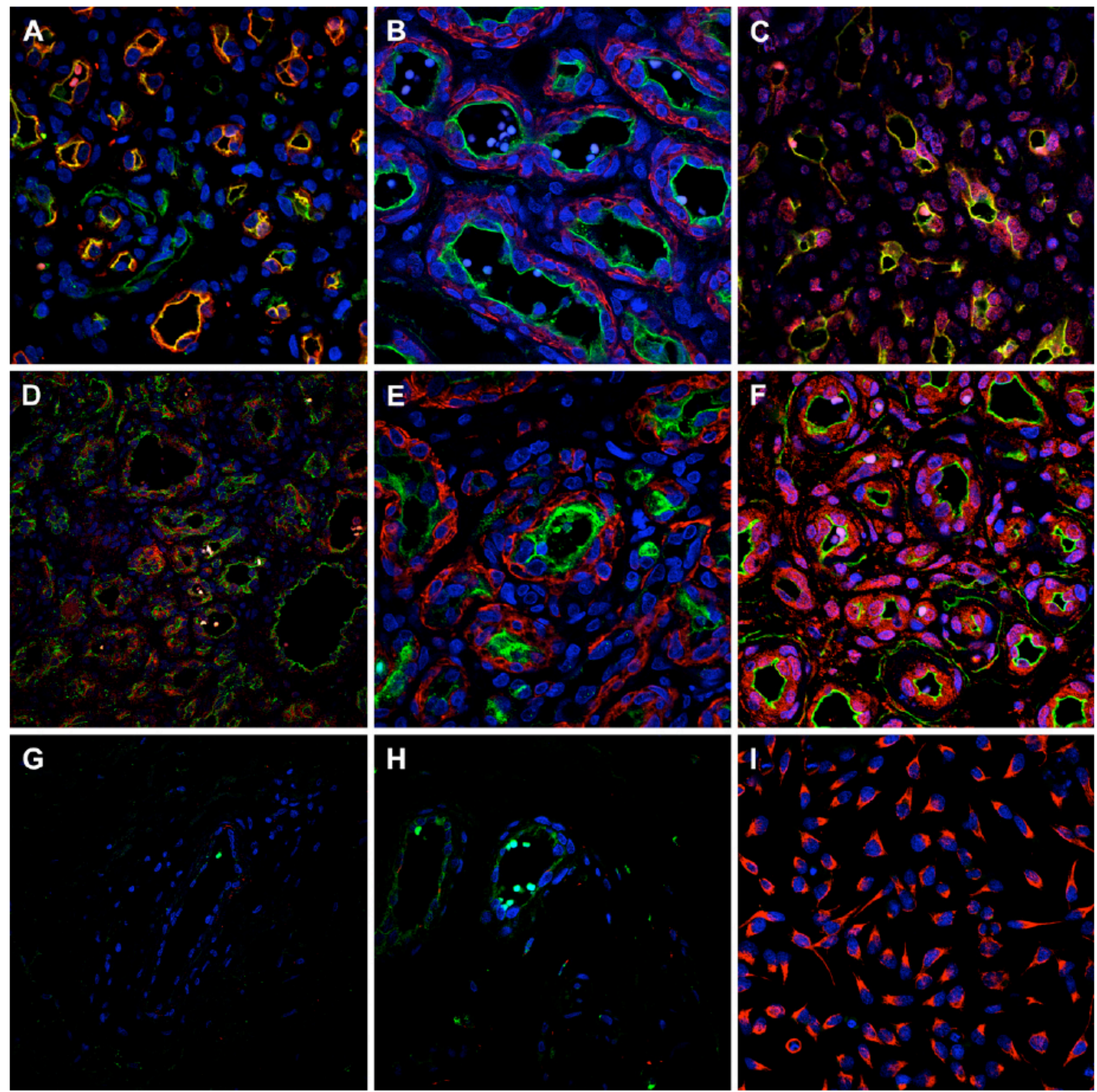

Figure 2 Immunohistochemical staining of representative paraffin sections of proliferating $(A-F)$ and involuted $(\mathrm{G}-\mathrm{H})$ infantile haemangiomas. A, GLUT-1 (red) and CD34 (green); B, CD34 (green) and SMA (red); C, CD34 (green) and VEGFR-2 (red); D, CD133 (red); E, ACE (green) and SMA (red); F, (green) and ATR2 (red); G, ACE (green) and ATR2 (red); H, CD31 (green) and CD133 (red) Original magnification $\times 400$. I, staining of HeLa cells for ATR2 (red) was taken at $\times 200$ magnification. Cell nuclei are counterstained with DAPI (blue). (For interpretation of the references to colour in this figure legend, the reader is referred to the web version of this article).

an in-vitro explant model previously described by Tan et $a l^{13}$ in 24-well plates (Raylab, Auckland, NZ). Cells were released from the solid matrix after 3 weeks in culture by treatment with 50 caseinolytic units/well of dispase (Becton-Dickson, Auckland, NZ) for 2 h at $37^{\circ} \mathrm{C}$. Dispase activity was quenched by diluting in MCDB medium (Sigma-Aldrich, Melbourne, Australia) containing 5\% Penstrep (Invitrogen), $0.5 \mu \mathrm{g} \mathrm{m}^{-1}$ Amphotericin B (Sigma-Aldrich) and 5\% foetal calf serum (Invitrogen) media at a dilution of 1:10. Viable cells were plated at a density of $1 \times 10^{5}$ cells per well, in triplicate, in a 96-well plate (Raylab, Auckland, NZ) and maintained with MCDB media as described above, but without the foetal calf serum. Angiotensin II (ATII) (Sigma-Aldrich) was added to a final concentration of 0 (controls), 25, 100 and $150 \mu \mathrm{g} \mathrm{ml}^{-1}$ and the cultures were maintained at $37{ }^{\circ} \mathrm{C}$ in $3 \% \mathrm{CO}_{2} / 97 \%$ air in a Heracell incubator (Kendo Laboratory Products, Langenselbold, Germany). The media was replaced every 3 days (with the appropriate amount of ATII added) for a total of 6 days. The number of blast-like structures in each well was counted using an Olympus IX51 inverted microscope (upgraded to IX71) fitted with phase contrast objectives and a colour view 1 camera (Tokyo, Japan).

\section{Immunocytochemistry}

The blast-like structures were collected from culture plates and cytospun onto slides using Cytospin ${ }^{\circledR} 3$ (Thermo Shanden, Cheshire, UK) prior to drying out on the bench for 


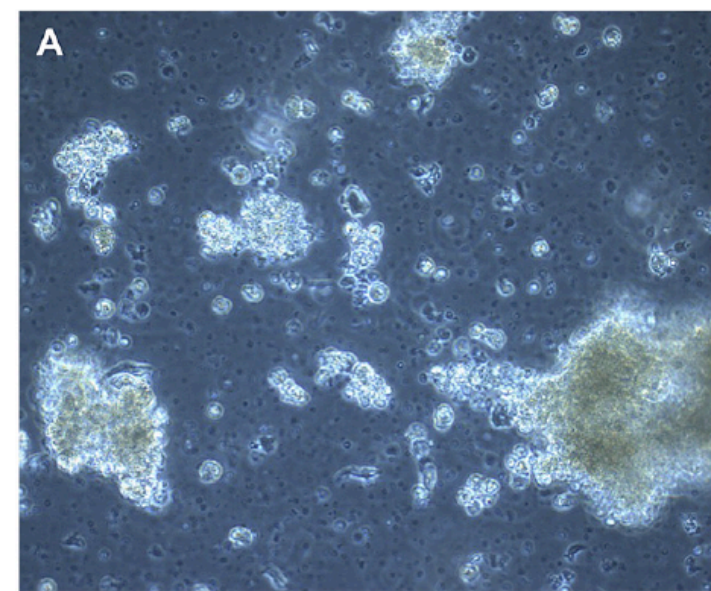

Blast Count vs Angiotensin II Concentrations At Day 6

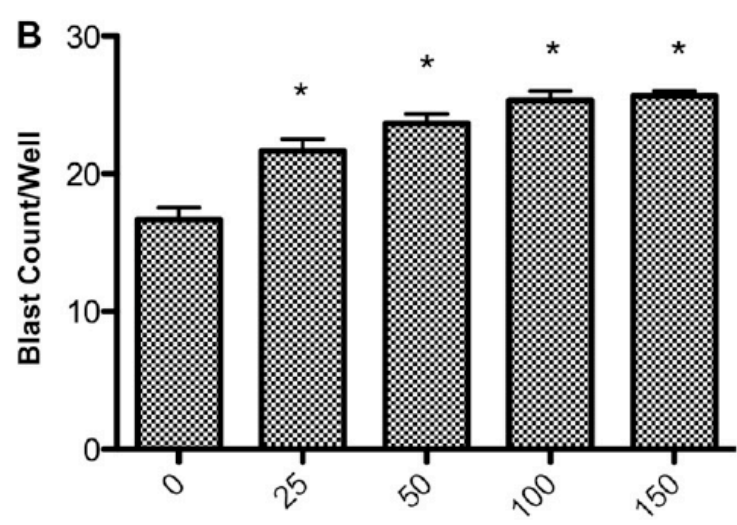

Angiotensin II Concentrations $\mu \mathrm{g} / \mathrm{ml}$

Figure 3 ATII induces blast formation. A, blast-like structures that form in culture in cells isolated from cultured haemangioma explants. B, that ATII stimulates blast formation in a dose dependent manner $\left({ }^{*} p<0.05\right)$.

$30 \mathrm{~min}$ at room temperature. Slides were blocked with TBST containing 5\% bovine serum albumin (Sigma-Aldrich) for $1 \mathrm{~h}$ prior to incubation overnight with primary antibodies (CD34, CD133, VEGFR-2 and ACE) diluted as for the immunohistochemistry (IHC) staining. Slides were washed in TBST and bound primary antibody detected with an appropriate secondary antibody (Goat anti-Mouse alexa-488 or Chicken anti-Rabbit alexa-594) prior to visualisation as for IHC.

\section{Image processing}

Images were captured using a Leica TCS 4D confocal laserscanning microscope fitted with a krypton/argon laser (Leica Lasertechnik, Heidelberg, Germany).

\section{Statistical analysis}

A one-way analysis of variance (ANOVA) with Bonferroni's post hoc test was used to test for an effect of ATII on cell growth. Changes where $p<0.05$ were considered significant.

\section{Results}

\section{Immunohistochemistry}

All proliferating infantile haemangioma obtained from the 10 patients showed immunoreactivity (IR) for GLUT-1 (Figure 2A, red), an established IHC marker used to differentiate infantile haemangioma from other vascular anomalies. ${ }^{14}$ Similarly, the endothelium of the haemangioma microvessels showed IR for the haematopoietic and endothelial cell marker CD34 (Figure $2 \mathrm{~A}-\mathrm{C}$, green), which was distinct from the pericyte layer identified by IR for SMA (Figure 2B, red) as previously reported..$^{1,3}$ The endothelium of the microvessels also showed IR for the endothelial markers, VEGFR-2 (Figure 2C, red) and CD31 (Figure 2D, green) as expected. Regions with IR for both VEGFR-2 and CD34 appear orange/yellow in the overlaid images.

The endothelium of these same microvessels also showed IR for ACE (Figure 2E, green) in contrast to the cells in the pericyte layer, which showed IR for SMA (Figure 2E red). In addition to expressing ACE, the endothelium also showed IR for ATR2. Figure 2F shows an overlay of staining for CD34 (green) to identify the endothelium, and ATIIR2 (red) staining. Involuted infantile haemangiomas showed minimal IR for ACE (Figure 2G, green) and ATR2 (Figure 2G, red) and diminished IR for CD133 (Figure 2H, red) compared with the proliferating haemangiomas (Figure 2E). These involuted lesions demonstrated IR for the endothelial marker, CD31 (Figure 2H, green) as expected.

Figure $2 \mathrm{~A}-\mathrm{F}$ thus demonstrates the co-expression of ATR2/CD34/CD31/VEGFR-2/CD133 in the cells of the endothelium of proliferating infantile haemangioma. IR for ATR1 was not detected on similarly processed haemangioma sections (data not shown). IHC staining of tissue that is known not to express ATR2 and ACE (uterine fibroid) and controls in which the primary antibodies were omitted showed minimal IR compared with the haemangioma test samples (data not shown). Specificity for ATR2 antibody was confirmed by positive staining of HeLa cells (Figure 2l, red).

\section{Response of haemangioma cell culture to ATII treatment}

Having demonstrated the expression of ATR2 by the cells forming the endothelium in proliferating infantile haemangioma, we sought to determine whether ATII was able to induce proliferation of cells extracted from explanted tissue as previous studies have shown that ATII can induce proliferation of $\mathrm{CD} 34^{+}$cells. Figure $3 \mathrm{~A}$ shows that treatment with ATII induces the formation of blast-like structures in cultured cells similar to those seen when haematopoietic progenitors were cultured on semi-solid media. Treatment with concentrations of 25, 50, 100 and $150 \mu \mathrm{g} \mathrm{ml}^{-1}$ ATII induced increased formation of blast-like structures in a dosedependent manner $(p<0.05)$ (Figure $3 B)$.

\section{Immunocytochemistry}

To confirm that the blast-like structures obtained from infantile haemangioma cell cultures treated with ATII had 

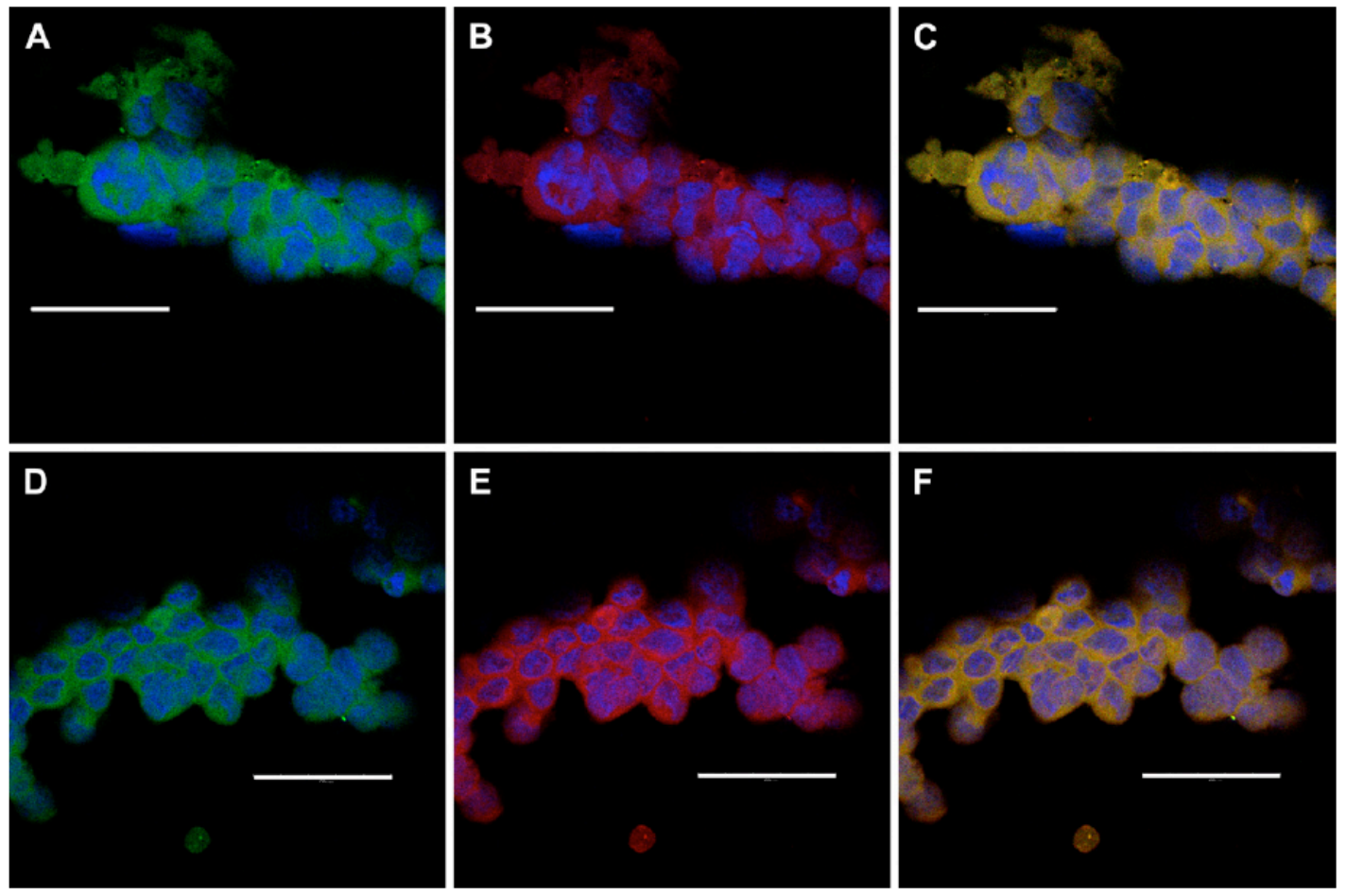

Figure 4 Immunocytochemical staining of blast-like structures. A\&B, IR for ACE (green) and VEGFR-2 (red) respectively; C, merged image indicating showing the cells co-expressing both ACE and VEGFR-2. D\&E, IR for CD34 (green) and CD133 (red) respectively; F, merged image indicating the cells co-express both CD34 and CD133. Cell nuclei are counterstained with DAPI (blue). Scale bars are $50 \mu \mathrm{m}$. (For interpretation of the references to colour in this figure legend, the reader is referred to the web version of this article)

the same phenotype as the cells forming the endothelium within proliferating infantile haemangioma, staining for ACE and VEGFR-2 was undertaken on the blast-like structures. The cells within the structures showed IR for ACE (Figure 4A, green) and VEGFR-2 (Figure 4B, red), with the overlaid image in Figure $4 C$ showing the cells expressing both markers. These same cells also demonstrated IR for CD34 (Figure 4D, green) and for CD133 (Figure 4E, red).

\section{Discussion}

Our immunofluorescence data show that proliferating infantile haemangioma expresses two essential components of the RAS, namely ACE and ATR2. This is the first report demonstrating the expression of ACE and ATR2 in the cells of proliferating infantile haemangioma.

The CD34, CD133 and VEGFR-2 staining patterns in our study confirm previous reports of the presence of this population of cells, previously referred to as EPCs, within proliferating infantile haemangioma. ${ }^{15}$ It has been previously shown that EPCs have the morphological appearance of colonies of cells when grown in culture. ${ }^{16}$ Taken together with the expression of CD34/CD133/VEGFR-2, we infer that the blast-like colonies that form from cells cultured from explants have an EPC-like phenotype. The capillary-like structures emanating from the infantile haemangioma explants grown in semi-solid media are consistent with an endothelial phenotype and the differentiation potential of EPCs. ${ }^{17}$ The $\mathrm{CD} 34^{+}$population of cells has been previously shown to proliferate in the presence of $\mathrm{ATII}^{18,19}$ and we have shown increased blast counts with increasing concentrations of ATII.

As white adipose tissue is a significant source of both local and circulating angiotensinogen, and it also contains all the components of the RAS, ${ }^{20}$ we speculate that the RAS may modulate the natural progression of infantile haemangioma. Figure 5 illustrates a potential model for the proposed interactions and functioning of the RAS in infantile haemangioma. We have shown that the $\mathrm{CD}_{3} 4^{+}$/ CD $133^{+} /$VEGFR $-2^{+}$cells present within the immature capillaries of proliferating infantile haemangioma are responsive to ATII, as they express ATR2 and ATII induces blast formation. We suggest that $\beta$-blockers, acting primarily on the kidney, reduce renin activity, ${ }^{21}$ thereby decreasing the conversion of angiotensinogen to angiotensin I, and ultimately to ATII by ACE. High levels of renin are found in infants (compared with adults) ${ }^{21}$ Caucasians (compared with blacks), ${ }^{22}$ females (compared with males) ${ }^{23}$ and premature (compared with full-term) infants. ${ }^{24}$ We propose that the high renin levels in these groups, coupled with the local expression of ACE in proliferating infantile haemangioma, sustain a high local concentration of ATII that drives proliferation. Mesenchymal stem cells, which 


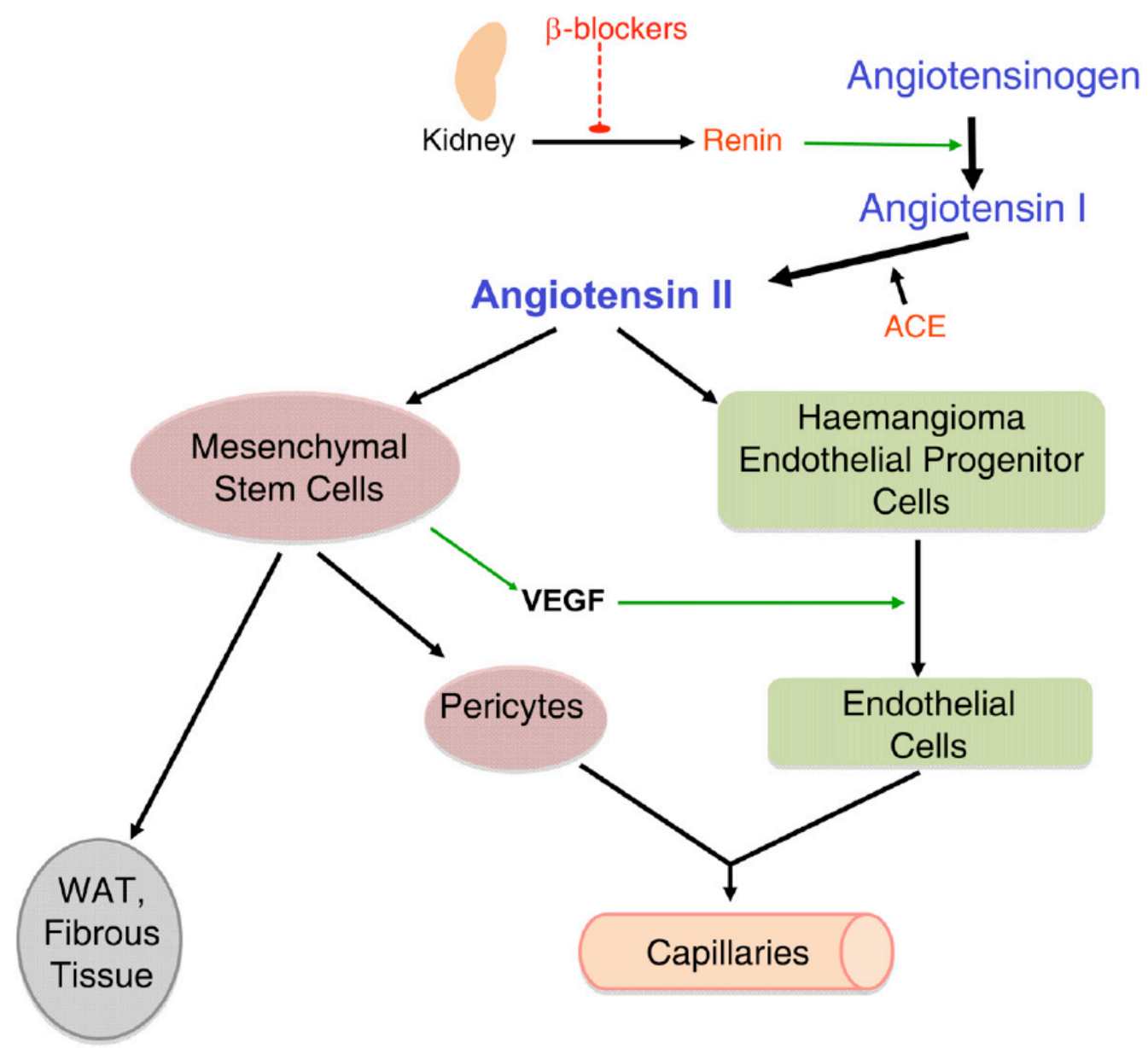

Figure 5 A model for infantile haemangioma showing regulation of endothelial progenitor cell proliferation and differentiation by the renin-angiotensin system (see text).

are present in infantile haemangioma, ${ }^{16}$ when stimulated with ATII secrete vascular endothelial growth factor (VEGF). ${ }^{25}$ We reason that high levels of VEGF, together with ATII, drive both the proliferation and differentiation of the EPCs into the mitotically active endothelial cells that characterise infantile haemangioma.

Treatment with $\beta$-blockers such as propranolol results in decreased ATII and VEGF production favouring involution, rather than proliferation. The trigger for spontaneous involution of infantile haemangioma may be due to a combination of either depletion of stem cells/progenitor cells, and/or the rapid decline of renin activity that occurs at the end of infancy. ${ }^{26}$ Late in the involuting phase, the mesenchymal stem cells differentiate into adipocytes and the fibrous tissue characteristic of involuted lesions.

The RAS can thus account for both the action of $\beta$-blockers and the process of spontaneous involution of infantile haemangioma. The role of the RAS in infantile haemangioma is supported by the clinical observation of a higher incidence of haemangioma in premature babies, female infants and in the Caucasian population. ${ }^{1}$ Premature birth is associated with higher circulating renin activity, ${ }^{24}$ black infants have lower renin levels than white infants, ${ }^{22}$ and female infants have higher renin levels than agematched males. ${ }^{23}$ Our model for infantile haemangioma progression represents a significant shift in thinking with regard to the biology of EPCs and stem cells in angiogenesis. The model for the action of $\beta$-blockers has obvious implications for the role of other anti-hypertensive agents such as ACE inhibitors and angiotensin receptor blocking drugs for the treatment of proliferating infantile haemangioma. In addition, our model predicts that these same antihypertensives will inhibit angiogenesis and vasculogenesis through blocking proliferation of $\mathrm{CD} 34^{+} / \mathrm{CD}_{133^{+}} / \mathrm{VEGFR}-2^{+}$ endothelial precursor cells and predicts a potential role of inhibitors of ACE and ATR2 in the treatment of proliferating infantile haemangioma. The ability of proliferating haemangioma to form blast colonies with an EPC phenotype, coupled with our recent data showing the involvement of primitive mesoderm with a neural crest phenotype $\mathrm{e}^{27}$ and downstream haemogenic endothelium ${ }^{28}$ in the biology of infantile haemangioma, suggest that this tumour is 
a developmental disorder of primitive mesodermal proliferation and differentiation governed by RAS.

\section{Conflict of interest}

None.

\section{Funding}

None.

\section{References}

1. Takahashi K, Mulliken JB, Kozakewich HP, et al. Cellular markers that distinguish the phases of hemangioma during infancy and childhood. J Clin Invest 1994;93:2357-64.

2. North PE, Waner M, Mizeracki A, et al. A unique microvascular phenotype shared by juvenile hemangiomas and human placenta. Arch Dermatol 2001;137:559-70.

3. Smoller BR, Alfelberg DB. Inf antile (juvenile) capillary hemangioma: A tumor of heterogeneous cellular elements. J Cutan Pathol 1993;20:330-6.

4. Tan ST, Velickovic M, Ruger BM, et al. Cellular and extracellular markers of hemangioma. Plast Reconstr Surg 2000;106:529-37.

5. Bennett ML, Fleischer Jr AB, Chamlin SL, et al. Oral corticosteroid use is effective for cutaneous hemangiomas: an evidence-based evaluation. Arch Dermatol 2001;137:1208-13.

6. Boon LM, MacDonald DM, Mulliken JB. Complications of systemic corticosteroid therapy for problematic hemangioma. Plast Reconstr Surg 1999;104:1616-23.

7. Drolet BA, Esterly NB, Frieden IJ. Hemangiomas in children. $N$ Engl J Med 1999;341:173-81.

8. Leaute-Labreze C, Dumas de la Roque E, Hubiche T, et al. Propranolol for severe hemangiomas of infancy. $N$ Engl $J$ Med 2008;358:2649-51.

9. Lisa B, Umesh D, Gresham TR. Propranolol for airway hemangiomas: case report of novel treatment. Laryngoscope 2009; 9999. NA.

10. Sans V, de la Roque ED, Berge J, et al. Propranolol for severe infantile hemangiomas: follow-up report. Pediatrics 2009;124: e423-431.

11. Denoyelle F, Leboulanger $N$, Enjolras $O$, et al. Role of propranolol in the therapeutic strategy of infantile laryngotracheal hemangioma. Int J Pediatr Otorhinolaryngol 2009;73: $1168-72$.
12. Tan ST, Itinteang T, Leadbitter P. Low dose propranolol for infantile haemangioma. JPRAS 2010; doi:10.1016/j.bjps.2010.06.010.

13. Tan $S T$, Hasan $Q$, Velickovic $M$, et al. A novel in vitro human model of hemangioma. Modern Pathol 2000;13:92-9.

14. North PE, Waner M, Mizeracki A, et al. Glut1: A newly discovered immunohistochemical marker for juvenile hemangiomas. Human Pathol 2000;31:11-22.

15. Yu Y, Flint AF, Mulliken JB, et al. Endothelial progenitor cells in infantile hemangioma. Blood 2004; 103:1373-5.

16. Hatzopoulos AK, Folkman J, Vasile E, et al. Isolation and characterization of endothelial progenitor cells from mouse embryos. Development 1998;125:1457-68.

17. Peichev M, Naiyer AJ, Pereira D, et al. Expression of VEGFR-2 and AC133 by circulating human CD34 + cells identifies a population of functional endothelial precursors. Blood 2000;95: 952-8.

18. Heringer-Walther S, Eckert K, Schumacher S-M, et al. Angiotensin-(1-7) stimulates hematopoietic progenitor cells in vitro and in vivo. Haematologica 2009;94:857-60.

19. Zambidis ET, Park TS, Yu W, et al. Expression of angiotensinconverting enzyme (CD143) identifies and regulates primitive hemangioblasts derived from human pluripotent stem cells. Blood 2008; 112:3601-14.

20. Saint-Marc P, Kozak LP, Ailhaud G, et al. Angiotensin II as a trophic factor of white adipose tissue: stimulation of adipose cell formation. Endocrinology 2001;142:487-92.

21. Dillon MJ, Rajani KB, Shah V, et al. Renin and aldosterone response in human newborns to acute change in blood volume. Arch Dis Child 1978;53:461-7.

22. Youmbissi TJ, Tedong F, Fairbank ST, et al. Plasma renin activity studies in a group of African neonates and children. J Trop Pediatr 1990;36:128-30.

23. Pipkin FB, Smales OR, O'Callaghan MJ. Renin and angiotensin levels in children. Arch Dis Child 1981;56:298-302.

24. Stephenson TJ, Pipkin FB, Elias-Jones AC. Factors influencing plasma renin and renin substrate in premature infants. Arch Dis Child 1991;66:1150-4.

25. Shi R-Z, Wang J-C, Huang $\mathrm{S}-\mathrm{H}$, et al. Angiotensin II induces vascular endothelial growth factor synthesis in mesenchymal stem cells. Exp Cell Res 2009;315:10-5.

26. Dillon MJ, Ryness JM. Plasma renin activity and aldosterone concentration in children. BMJ 1975;4:316-9.

27. Itinteang T, Tan ST, Brasch H, Day DJ. Primitive mesodermal cells with a neural crest stem cells phenotype predominate proliferating haemangioma. J Clin Pathol; 2010; doi: 10.1016/j.bjps.2010.06.010.

28. Itinteang T, Tan ST, Brasch $\mathrm{H}$, et al. Haemogenic endothelium in infantile haemangioma. J Clin Pathol 2010 (accepted). 


\subsection{Controls}
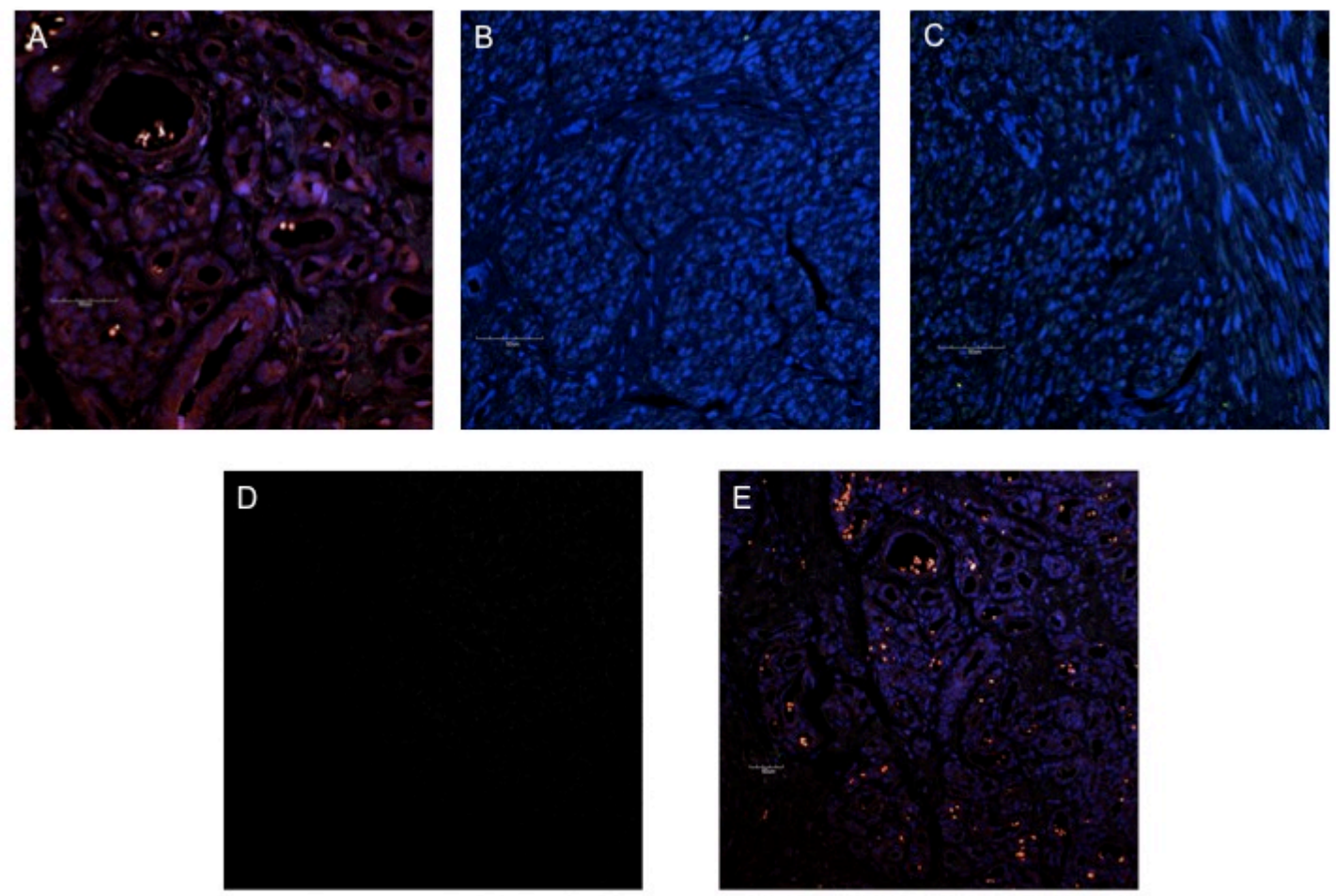

Control Figures demonstrating immunoreactivity (IR): for Angiotensin II receptor 2 (A, red) and minimal IR for Angiotensin II receptor 1 (A, green) in a proliferating IH specimen; absence of IR for Angiotensin Converting Enzyme (ACE) and Angiotensin II receptor 2 (B \& C, red respectively) in human uterine fibroid specimens. Appropriate negative controls with ommission of the primary antibodies and with use of the alexa flour 488 and 594 only (D \& E, respectively) in proliferating IH specimens. All specimens were counterstained with DAPI to identify the cell nuclei. 


\section{Chapter 9: Embryonic Stem Cell Markers in Infantile Haemangioma}

\subsection{Introduction and Overview}

We have previously proposed an ESC origin for $\mathrm{IH}$, as discussed in Chapter 3 of this thesis. This has led us to further investigate the expression of ESCassociated markers within $\mathrm{IH}$.

ESCs are pluripotent cells typically derived from the inner cell mass of the blastocyst ${ }^{78,79}$, and the identification of the unique signature profile of ESCs has enabled the demonstration of the expression of certain proteins associated with an ESC phenotype. Further characteristics of ESCs are their ability to form all three germ layers in vitro, and teratomas in vivo ${ }^{80}$.

Proteins associated with ESCs include Oct-4, a POU transcription factor which has been demonstrated in pluripotent primitive embryonic cells ${ }^{81}$; STAT-3, a signal transducer and activator transcription factor critical in ESC renewal ${ }^{82}$; SSEA-4, a cell surface marker associated with ESCs ${ }^{78}$; and Nanog, a transcription factor crucial for the maintenance of ESC pluripotency ${ }^{83}$.

In this chapter I report the expression of Oct-4, STAT-3 and SSEA-4 on the endothelium of the capillaries within proliferating IH. Interestingly Nanog is not expressed by the cells of the endothelium, but by cells in the interstitium.

The expression of only some, but not all ESC-associated markers on two different populations of cells within proliferating $\mathrm{IH}$ suggests a cellular origin of IH downstream of ESCs. The two possibilities discussed in this 
chapter to account for this finding are that the Nanog-positive cells are a derivation from the more primitive endothelium, or alternatively there may be two different populations of primitive cells in proliferating $\mathrm{IH}$.

These findings reveal novel primitive populations of stem cells within proliferating $\mathrm{IH}$, that are downstream of ESCs, as determined by the expression profile of ESC and confirmed by their inability to form teratomas in affected patients.

\subsection{Contribution}

All IHC experiments, apart from the stat-3, and drafting of the manuscript were performed by myself, and edited by my supervisors. The stat-3 experiment was performed by Dr Vishvanath and the RT-PCR experiements were performed by Dr Steel and $\mathrm{H}$ Best. 
British Journal of Dermatology

$$
\begin{aligned}
& \text { British Association } \\
& \text { of Dermatologists }
\end{aligned}
$$

Expression of Embryonic Stem Cell Markers in Infantile Haemangioma

\begin{tabular}{|r|l|}
\hline Journal: & British Journal of Dermatology \\
\hline Manuscript ID: & Draft \\
\hline Manuscript Type: & Original Article \\
\hline Author: & nubmitted by the \\
\hline Complete List of Authors: & $\begin{array}{l}\text { Itinteang, Tinte; Victoria University of Wellington, School of } \\
\text { Biological Sciences } \\
\text { Tan, Swee; Hutt Valley Hospital, Centre for the Study and } \\
\text { Treatment of Vascular birthmarks } \\
\text { Steel, Ryan } \\
\text { Best, Heather; Victoria University of Wellington, School of Biological } \\
\text { Sciences } \\
\text { Brasch, Helen; Department of Pathology, Hutt Hospital } \\
\text { Day, Darren; Victoria University of Wellington, School of Biological } \\
\text { Sciences }\end{array}$ \\
\hline Keywords: & $\begin{array}{l}\text { Infantile haemangioma, embryonic stem cells, haemogenic } \\
\text { endothelium, primitive mesoderm }\end{array}$ \\
\hline &
\end{tabular}

SCHOLARONE"

Manuscripts 


\section{Expression of Embryonic Stem Cell Markers in Infantile Haemangioma}

Short title: Embryonic stem cell markers in haemangioma

Authors: ${ }^{1-3} \mathrm{~T}$ Itinteang, ${ }^{1-4} \mathrm{ST}$ Tan,${ }^{1} \mathrm{R}$ Steel, ${ }^{1} \mathrm{HA}$ Best, ${ }^{1} \mathrm{~A}$ Vishvanath, ${ }^{3,5} \mathrm{HD}$ Brasch, ${ }^{1-}$ ${ }^{3}$ DJ Day

"equal senior authors

${ }^{1}$ School of Biological Sciences, Victoria University of Wellington; ${ }^{2}$ Centre for the Study \& Treatment of Vascular Birthmarks, Wellington Regional Plastic, Maxillofacial \& Burns Unit, Hutt Hospital; ${ }^{3}$ Gillies McIndoe Research Institute; ${ }^{4}$ Wellington School of Medicine \& Health Sciences, University of Otago; and ${ }^{5}$ Department of Pathology, Hutt Hospital, Wellington, New Zealand.

Corresponding author: Professor Swee T Tan, MBBS FRACS PhD, Wellington Regional Plastic, Maxillofacial \& Burns Unit, Hutt Hospital, High Street, Private Bag 31-907, Lower Hutt, New Zealand.

Tel: +64 45872506 Fax: +64 45872510 Em: swee.tan@huttvalleydhb.org.nz

Funding statement: This work is supported by grants from the Wellington Regional Plastic Surgery Unit Research \& Education Trust; the Wellington Medical Research Foundation; the Surgical Research Trust, Pub Charities and the Cancer Society of New Zealand. Dr Vishvanath was supported, in part, by the Cancer Society of New 
Zealand. Dr Itinteang was supported by the Royal Australasian College of Surgeons' Foundation for Surgery Scholarship. None of these funders was involved in the research and its publication, or for the publication.

Disclosures: The authors have no competing financial interest or any other conflict of interest.

Manuscript count: word (1609), figures (2), tables (0).

What is already known about this topic?

A primitive mesodermal phenotype origin of infantile haemangioma $(\mathrm{IH})$ with the ability to differentiate down erythropoietic and terminal mesenchymal lineages.

What does this study add?

The unique expression of human embryonic stem cell (hESC) associated transcription factors on two different populations of cells in proliferating $\mathrm{IH}$ suggests a cellular origin downstream of hESC.

Aspects of this work were presented at the Australian and New Zealand Head \& Neck Cancer Society, Manly, Australia, September 2-4, 2010; the American Society of Plastic Surgeons' Meeting, Toronto, Canada, 1-5 October 2010; and the New Zealand Association of Plastic Surgeons' Annual Scientific Meeting, Auckland, New Zealand, 26 \& 27 November, 2010. 


\begin{abstract}
Background: The origin of infantile haemangioma $(\mathrm{IH})$ remains enigmatic. A primitive mesodermal phenotype origin of $\mathrm{IH}$ with the ability to differentiate down erythropoietic and terminal mesenchymal lineages has been demonstrated recently.

Objectives: To investigate the expression of human embryonic stem cell (hESC) markers in $\mathrm{IH}$.

Methods: Immunohistochemistry and real-time RT-PCR were used on IH biopsies to investigate the expression of hESC markers.

Results: The hESC markers, Oct-4, STAT-3, and SSEA-4 were collectively expressed on the endothelium of proliferating $\mathrm{IH}$ lesions while Nanog was only expressed by cells in the interstitium.

Conclusion: The novel finding of the expression of hESC on two different populations of cells in proliferating $\mathrm{IH}$ suggests a cellular origin downstream of hESC, for $\mathrm{IH}$.
\end{abstract}

Keywords: Infantile haemangioma, embryonic stem cells, haemogenic endothelium, primitive mesoderm 


\section{Introduction}

Infantile haemangioma $(\mathrm{IH})$, the most common tumour of infancy, affects about $10 \%$ of infants with a predilection for white, female and premature infants ${ }^{1-4}$. It typically undergoes rapid growth during infancy, characterised by the proliferation of immature capillaries that spontaneously involute to form a fibro-fatty residuum over the next 10 12 years $^{1-4}$.

We have recently shown that proliferating $\mathrm{IH}$ expresses brachyury, a marker of the primitive mesoderm, as well as markers associated with a neural crest phenotype ${ }^{5}$. We have proposed that this primitive mesoderm gives rise to a haemogenic endothelium phenotype characterised by the collective expression of CD133, angiotensin converting enzyme (ACE), GATA-2, Tal-1 and CD34 ${ }^{6}$. Additionally, in support of being a haemogenic endothelium, we have demonstrated that when $\mathrm{IH}$ biopsies are grown in vitro the cells emanating from the explants, express similar proteins as those of the endothelial layer, and have the ability to undergo primitive erythropoesis ${ }^{7}$ and mesenchymal differentiation ${ }^{8}$, and that peptides derived from angiotensinogen are crucial regulators of $\mathrm{IH}$ proliferation and differentiation ${ }^{9}$.

Human embryonic stem cells (hESCs) are pluripotent cells derived from the inner cell mass of blastocyst ${ }^{10,11}$ that have the ability to differentiate into all three embryonic germ layers. hESCs have been identified by the collective expression of transcription factors and cell surface receptors including Oct- $4^{12}$, STAT- $3^{13}$, stage specific embryonic antigen-4 (SSEA-4) $)^{14}$ and Nanog ${ }^{15}$. The presence of tumours derived from embryonic stem cells (ESC) is characterised by their ability to form cells of all three germ layers in vitro and teratoma in vivo ${ }^{16}$. As hESCs have been proposed as precursors of hemangioblasts ${ }^{17}$ that give rise to endothelial and 
haematopoietic stem cells, we proposed a model for $\mathrm{IH}$ with a hESC origin that gives rise to a. primitive mesoderm-derived haemogenic endothelium with a neural crest phenotype ${ }^{56}$

In this study we investigated the presence of hESC associated markers using immuno-histochemical staining and quantitative RT-PCR (qRT-PCR) in IH.

Materials and Methods

Tissues Biopsies

Biopsies of proliferating $\mathrm{IH}$ lesions affecting the skin and the underlying subcutaneous tissues in six patients (aged 3-10; mean, 6 months) and involuted lesions from three patients (aged 8, 9 and 11 years) and normal subcutaneous fat from two adults undergoing elective surgery were obtained according to a protocol approved by the Wellington Regional Ethics Committee.

$\underline{\text { Immunolabelling }}$

Biopsies of proliferating $\mathrm{IH}$ lesions from six patients and involuted $\mathrm{IH}$ lesions from three patients were formalin-fixed and paraffin embedded using standard procedures. $\mathrm{H} \& \mathrm{E}$ and IHC analyses were performed on $4 \mu \mathrm{m}$ serial sections and processed as previously described ${ }^{5,6,9}$. Immunolabelling was performed with primary antibodies specific for anti-human CD34 (1:200), GLUT-1 (1:300), SMA (1:400), Oct-4 (1:300), SSEA-4 (1:300), STAT-3 (1: 200), PCNA (1:100) and Nanog (1:300). Negative controls included staining of normal adult human subcutaneous fat, and omission of the primary antibodies when staining $\mathrm{IH}$ tissues.

\section{Quantitative RT-PCR}


For the analysis of relative mRNA expression of Oct- 4 and Nanog, RNA was extracted from fresh proliferating $\mathrm{IH}$ biopsies of five patients, aged 3-8 (mean, 5) months and qRT-PCR was performed using specific primers for Oct-4 and Nanog. Primers used for Oct- 4 were as previously reported ${ }^{18}$, whereas the primer sequence for Nanog were: forward GATTTGTGGGCCTGAAGAAA, reverse AAGTGGGTTGTTTGCCTTTG. Expression relative to that for glyceraldhye 3phosphate dehydrogenase (GAPDH) is reported as $\triangle \mathrm{CT}$ values. Data was collected during the extension step and melt-curve analysis and Agarose gel electrophoresis was performed to confirm the specificity of amplification (data not shown).

\section{Results}

\section{Immunohistochemistry}

The endothelium of all proliferating IHs was immunoreactivity (IR) for the endothelial associated marker, CD34 (Figures $1 \mathrm{~A}-\mathrm{C}$ ) and the CD34+ cells were surrounded by an outer concentric pericyte layer showing IR for smooth muscle actin (SMA) as previously described ${ }^{5,6,9,19}$. The endothelium of all IH lesions also showed IR for the IH marker, GLUT-1, as expected ${ }^{6,20}$ (data not shown).

Expression of the hESC associated markers Oct-4, STAT-3, SSEA-4 and Nanog was investigated in proliferating and involuted IHs. Figure 1 demonstrates representative proliferating IH sections showing strong IR for Oct-4, STAT-3, SSEA-4 and Nanog. Figure 1A, shows that the $\mathrm{CD} 34^{+}$cells (green) that form the endothelium also show strong IR for Oct-4 (red). IR for SSEA-4 (green) is also localised to the endothelium and is sparse on the cells in the interstitium (Figure 1B). However, only a sub-population of cells within the interstitium show strong IR for Nanog (Figure 1B, red). Figure $1 \mathrm{C}$ confirms that the cells that stain intensely for Nanog (red) are not 
those of the endothelium that stain positively for CD34 (green). Figure 1D shows that IR for STAT-3 (red) is predominantly localised in the cells that form the endothelium of the microvessels. Regions that show the strongest IR for STAT-3 also demonstrate the strongest IR for PCNA (green), indicating that STAT-3 is expressed in proliferative regions of IH. IR for PCNA is predominantly localised to the endothelium of the microvessels (yellow arrow heads). To determine whether the expression of these ESC-associated markers are lost as the tumour involutes, staining of involuted $\mathrm{IHs}$ was undertaken using the same parameters for IHC used for the proliferating $\mathrm{IHs}$. Figures $1 \mathrm{E} \& \mathrm{~F}$ show that there are fewer $\mathrm{CD} 34^{+}$microvessels (green staining) and minimal IR for Nanog (Figure 1E, red) and Oct-4 (Figure 1F, red). As with involuted IHs, IR for CD34 (green) was detected in normal adult subcutaneous fat which showed no IR for Nanog (Figure 1G, red)

Figures $1 \mathrm{~A}-\mathrm{C}$ shows expression of $\mathrm{CD} 34$ (green) that identifies the endothelium of the capillaries of proliferating $\mathrm{IH}$. Figures $1 \mathrm{~A} \& \mathrm{~B}$ show that the CD34+ endothelium also expresses hESC markers, Oct-4 (red) and STAT-3, respectively (red). Figure $1 \mathrm{C}$ demonstrates that cells showing IR for Nanog (red) are located in the interstitium but not the endothelium of the microvessels. Figure 1D confirms dual expression of the Oct-4 (green) and SSEA-4 (red) by cells of the capillary endothelium.

Figures 2 A\&B confirm that the expression of Oct- 4 and SSEA- 4 are localised to the endothelium by counterstaining with SMA to identify the pericyte layer. Figure 2A shows that the IR for Oct-4 (red) and SMA (green) do not overlap, similarly Figure $2 \mathrm{~B}$ shows that the endothelium stains for SSEA-4 (green) while the pericyte layer stains for SMA (red). 


\section{Quantitative RT-PCR}

To corroborate the staining presented in Figures 1 \& 2, qRT-PCR of mRNA isolated from proliferating $\mathrm{IH}$ tissues was undertaken. Expression levels for Oct-4 and Nanog were determined relative to that for glyceraldehyde dehyrogenase that is abundantly expressed within $\mathrm{IH}^{23}$. Both Oct-4 and Nanog were abundantly expressed with the relative expression for Oct- 4 being $\Delta \mathrm{Ct}=6.72$ and that for Nanog being $\Delta \mathrm{Ct}=1.51$.

\section{Discussion}

We have previously demonstrated the expression of primitive mesodermal (brachyury) $^{5}$ and primitive haematopoietic-associated (CD133, ACE, GATA-2, Tal-1, and EPO-R $)^{6,7}$ markers and embryonic-associated haemoglobin $(\zeta \text { chain })^{7}$ on the haemogenic endothelium of capillaries of proliferating $\mathrm{IH}$ and that the mature haematopoietic associated marker, CD45, is only expressed by cells in the interstitium $^{6}$. We have also shown that this haemogenic endothelium possesses the ability to undergo primitive erythropoesis in vitro ${ }^{7}$ and mesenchymal differentiation ${ }^{8}$.

The isolation of hESCs from the inner cell mass of the blastocyst has enabled the identification and study of the pluripotent nature of hESCs ${ }^{11}$. Oct- 4 , also known as POU5F1, is a mammalian POU transcription factor, which is expressed by pluripotent early embryonic cells ${ }^{12}$. STAT-3, a member of the signal transducer and activator transcription factor family, has been shown to be crucial in ESC renewal and blockage of differentiation ${ }^{13}$. SSEA-4 is a cell surface marker expressed by hESCs ${ }^{11}$. Nanog, a homeobox-containing transcription factor, is essential for the maintenance of pluripotency ${ }^{15}$. The hallmark of ESC-derived tumors is their ability to form teratoma both in vivo and all three germ layers in vitro ${ }^{16}$. 
To the best of our knowledge this is the first report demonstrating the expression pattern of ESC-associated markers in $\mathrm{IH}$. It is interesting that the expression of Oct-4, STAT-3 and SSEA-4 are localised to the endothelium of the capillaries in proliferating $\mathrm{IH}$. It is equally intriguing that the expression of Nanog is limited only to cells in the interstitium, away from the endothelium. We hypothesise that the endothelium of proliferating $\mathrm{IH}$ is derived from hESC-phenotypic cells, and that the expression of the more primitive ESC-associated marker, Oct-4, resemble the most primitive population within proliferating $\mathrm{IH}$, and which gives rise to cells expressing the down-stream transcription factor, Nanog ${ }^{15}$. Alternatively there maybe two different hESC-phenotypic populations within proliferating IH lesions, one giving rise to the haemogenic endothelium and the other to the Nanog+ population in the interstitium. It is equally intriguing that $\mathrm{IH}$ derived cells do not possess the ability to form teratoma in SCID/NOD mice, as shown by Khan et al in which CD133+ proliferating $\mathrm{IH}$ derived cells were used ${ }^{21}$. We have previously shown that the $\mathrm{CD} 133^{+}$ cells are localised to the endothelium of the capillaries of proliferating $\mathrm{IH}^{5,6,9}$, and this study confirms the expression of the hESC markers Oct-4, SSEA-4 and STAT-3, but not Nanog, by the same population of cells. Results of these experiments are further supported by the clinical observation of the absence of teratoma formation in patients affected by $\mathrm{IH}^{22}$. We therefore conclude that the expression of some, but not all, of the hESC markers used in this study by a single cell population in proliferating $\mathrm{IH}$ does not indicate the presence of true ESCs. It appears that this population of cells resemble a phenotype at a differentiation stage downstream of hESCs, and that these downstream cells may reflect the true origin of $\mathrm{IH}$, although this is currently the topic of further investigation. 
The novel identification of hESC markers within proliferating $\mathrm{IH}$ provides further evidence that $\mathrm{IH}$ is a developmental anomaly. The $10 \%$ incidence of $\mathrm{IH}$ in the Caucasian population implies a potential source of ex-utero cells downstream of hESCs, and IH as a unique model for studying human development.

\section{Acknowledgments}

This work is supported by grants from the Wellington Regional Plastic Surgery Unit Research \& Education Trust; the Wellington Medical Research Foundation; the Surgical Research Trust, Pub Charities and the Cancer Society of New Zealand. Dr Vishvanath was supported, in part, by the Cancer Society of New Zealand. Dr Itinteang was supported by the Royal Australasian College of Surgeons' Foundation for Surgery Scholarship. 


\section{References}

1. Tan ST, Velickovic M, Ruger BM, Davis PF. Cellular and extracellular markers of hemangioma. Plast Reconstr Surg. 2000;106:529-537.

2. Frieden IJ, Eichenfield LF, Esterly NB, Geronemus R, Mallory SB, the Guidelines/Outcomes C. Guidelines of care for hemangiomas of infancy. J Am Acad Dermatol. 1997;37:631-637.

3. Takahashi K, Mulliken JB, Kozakewich HP, Rogers RA, Folkman J, Ezekowitz RAB. Cellular markers that distinguish the phases of hemangioma during infancy and childhood. J Clin Invest. 1994;93:2357-2364.

4. Tan ST, Itinteang T, Leadbitter P. Low-dose propranolol for infantile haemangioma. J Plast Reconstr Aesth Surg. doi:10.1016/j.bjps.2010.06.010.

5. Itinteang T, Tan ST, Brasch H, Day DJ. Primitive mesodermal cells with a neural crest stem cell phenotype predominate proliferating infantile haemangioma. J Clin Pathol. 2010;63:771-776.

6. Itinteang T, Tan ST, Brasch H, Day DJ. Haemogenic endothelium in infantile haemangioma. J Clin Pathol. 2010;63:982-986.

7. Itinteang T, Tan ST, Brasch HD, Vishvanath A, Day DJ. Primitive erythropoiesis in infantile haemangioma. Brit J Dermatol. 2011 (Accepted).

8. Itinteang T, Vishvanath A, Day DJ, Tan ST. Mesenchymal stem cells in infantile haemangioma. J Clin Pathol. 2011; doi:10.1136/jcp.2010.085209.

9. Itinteang T, Brasch HD, Tan ST, Day DJ. Expression of components of the reninangiotensin system in proliferating infantile haemangioma may account for the propranolol-induced accelerated involution. J Plast Reconstr Aesth Surg. doi:10.1016/j.bjps.2010.08.039.

10. Thomson JA, Itskovitz-Eldor J, Shapiro SS, et al. Embryonic stem cells lines derived from human blastocysts. Science. 1998;282:1145-1147.

11. Laslett AL, Filipczyk AA, Pera MF. Characterization and culture of human embryonic stem cells. Trends Cardiovasc Med. 2003;13:295-301.

12. Nichols J, Zevnik B, Anastassiadis K, et al. Formation of pluripotent stem cells in the mammalian embryo depends on the POU transcription factor Oct4. Cell. 1998;95:379-391.

13. Burdon T, Smith A, Savatier P. Signalling, cell cycle and pluripotency in embryonic stem cells. Trends Cell Biol. 2002;12:432-438.

14. Brimble SN, Sherrer ES, Uhl EW, et al. The cell surface glycosphingolipids SSEA-3 and SSEA-4 are not essential for human ESC pluripotency. John Wiley \& Sons, Ltd; 2007:25;54-62.

15. Rodda DJ, Chew J-L, Lim L-H, et al. Transcriptional regulation of Nanog by OCT4 and SOX2. J Biol Chem. 2005;280:24731-24737.

16. Xu C, Inokuma MS, Denham J, et al. Feeder-free growth of undifferentiated human embryonic stem cells. Nat Biotech. 2001;19:971-974.

17. Kennedy M, D'Souza SL, Lynch-Kattman M, Schwantz S, Keller G. Development of the hemangioblast defines the onset of hematopoiesis in human ES cell differentiation cultures. Blood. 2007;109:2679-2687.

18. Avery S, Zafarana G, Gokhale PJ, Andrews PW. The role of SMAD4 in human embryonic stem cell self-renewal and stem cell fate. Stem Cells; 2010;28:863-873.

19. Tan ST, Hasan Q, Velickovic M, Ruger BM, Davis RPF, Davis PF. A novel in vitro human model of . Modern Pathol. 2000;13:92-99. 
20. Williams RL, Risau W, Zerwes H-G, Drexler H, Aguzzi A, Wagner EF. Endothelioma cells expressing the polyoma middle $\mathrm{T}$ oncogene induce hemangiomas by host cell recruitment. Cell. 1989;57:1053-1063.

21. Smoller BR, Alfelberg DB. Infantile (juvenile) capillary hemangioma: A tumor of heterogeneous cellular elements. J Cutan Pathol. 1993;20:330-336.

22. North PE, Waner M, Mizeracki A, Mihm MC. Glut1: A newly discovered immunohistochemical marker for juvenile hemangiomas. Human Pathol. 2000;31:1122.

23. Khan ZA, Boscolo E, Picard A, et al. Multipotential stem cells recapitulate human infantile hemangioma in immunodeficient mice. J Clin Invest. 2008;118:2592-2599.

24. Mulliken JB, Glowacki J. Hemangiomas and vascular malformations in infants and children: A classification based on endothelial characteristics. Plast Reconstr Surg. 1982;69:412-419.

25. Yu Y, Varughese J, Brown LF, Mulliken JB, Bischoff J. Increased Tie2 expression, enhanced response to angiopoietin-1, and dysregulated angiopoietin-2 expression in hemangioma-derived endothelial cells. Am J Pathol. 2001;159:22712280. 


\section{Figure Legends}

Figure 1. Immunohistochemical staining of $\mathrm{IH}$ for hESC markers. Panels A-D show staining of representative sections of proliferating IHs. Panel A, the endothelium of vessels are immunoreactive (IR) for CD34 (green staining). The same $\mathrm{CD}_{4} 4^{+}$cells are also IR for Oct-4 (red staining). Panel B, the cells of the endothelium are IR for SSEA-4 (green staining) but not for Nanog (red staining). A sub-population of cells within the interstitium show IR for Nanog but do not stain for SSEA-4. Panel C shows staining for CD34 (green) to unequivocally identify the endothelium, and Nanog (red staining). The staining in panel $\mathrm{C}$ corroborates the staining pattern present in panel $\mathrm{B}$ as none of red Nanog ${ }^{+}$cells are IR for CD34. Panel D shows staining for the transcription factor, STAT-3 (red staining) and the proliferation marker PCNA (green) that identifies the nuclei of replicating and recently replicated cells. STAT-3 is expressed predominately on the endothelium, with weaker IR being detected on cells within the interstitium. The PCNA IR nuclei are also predominantly found on the cells of the endothelium. Selective doubly-stained STAT-3 and PCNA IR nuclei are indicated with yellow arrow heads. Panels E\&F show staining of a representative involuted IH lesion. Panel E shows staining for CD34 (green) and Nanog (red). Larger blood vessels with a CD $34^{+}$endothelium are readily detected as expected but no Nanog IR is detected when compared with the Nanog staining of proliferating IH presented in Panels B\&C. Similarly, Panel F shows staining for CD34 (green) and Oct-4 (red) in an involuted IH lesion. No Oct-4 IR is detected compared with that seen in proliferating lesions as described in Panel A. Panels G\&H show staining for CD34 (green) and Nanog (red, panel G) and Oct-4 (red, panel H) on normal adult subcutaneous fat. The staining pattern for the adult subcutaneous fat is very similar to the involuted IH samples presented in Panels E\&H, and is markedly different from 
that seen in the proliferating lesions presented in Panels A-C. Cell nuclei are counterstained with DAPI (blue) in all panels. The scale bar is $10 \mathrm{~mm}$ for all panels except panel $\mathrm{D}$ on which it is $20 \mathrm{~mm}$.

Figure 2. The pericyte layer in proliferating IH does not express Oct-4 or SSEA-4. Panels A\&B show representative $\mathrm{IHC}$ staining of proliferating $\mathrm{IH}$ in which the pericyte layer is identified by IR for smooth muscle actin (SMA, green staining). Panel A shows that the SMA+ pericyte layer (green staining) shows minimal IR for Oct-4 (red staining) but the endothelium shows strong IR for Oct-4 consistent with the data presented in Figure 1, Panel A. Similarly, the IR for SSEA-4 is greatest on the endothelium with only weak staining being detected on the $\mathrm{SMA}^{+}$(red staining) pericyte layer. Cell nuclei are counterstained with DAPI (blue) in both panels. Scale bars are $10 \mathrm{~mm}$. 


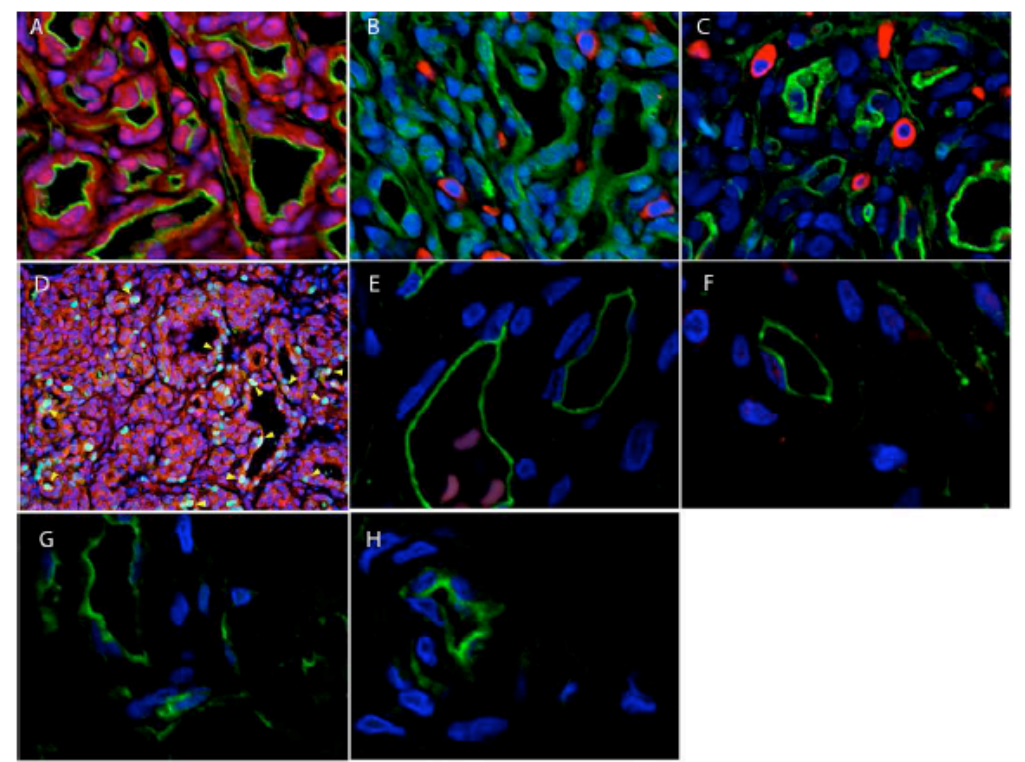

Figure 1

Immunohistochemical staining of IH for hESC markers. Panels A-D show staining of representative sections of proliferating IHs. Panel A, the endothelium of vessels are immunoreactive (IR) for CD 34 (green staining). The same CD34+ cells are also IR for Oct-4 (red staining). Panel B, the cells of the endothelium are IR for SSEA-4 (green staining) but not for Nanoq (red staining). A sub-population of cells within the interstitium show IR for Nanog but do not stain for SSEA-4. Panel C shows staining for CD34 (green) to unequivocally identify the endothelium, and Nanog (red staining). The staining in panel $\mathrm{C}$ corroborates the staining pattern present in panel B as none of red Nanog + cells are IR for CD 34. Panel D shows staining for the transcription factor, STAT-3 (red staining) and the proliferation marker PCNA (green) that identifies the nuclei of replicating and recently replicated cells. STAT-3 is expressed predominately on the endothelium, with weaker IR being detected on cells within the interstitium. The PCNA IR nuclei are also predominantly found on the cells of the endothelium. Selective doubly-stained STAT-3 and PCNA IR nuclei are indicated with yellow arrow heads. Panels E\&F show staining of a representative involuted IH lesion. Panel E shows staining for

CD34 (green) and Nanog (red). Larger blood vessels with a CD34+ endothelium are readily detected as expected but no Nanog IR is detected when compared with the Nanog staining of proliferating IH presented in Panels B\&C. Similarly, Panel F shows staining for CD 34 (green) and

Oct-4 (red) in an involuted IH lesion. No Oct-4 IR is detected compared with that seen in proliferating lesions as described in Panel A. Panels G\&H show staining for CD34 (green) and Nanog (red, panel G) and Oct-4 (red, panel H) on normal adult subcutaneous fat. The staining pattern for the adult subcutaneous fat is very similar to the involuted IH samples presented in Panels E\&H, and is markedly different from that seen in the proliferating lesions presented in Panels A-C. Cell nuclei are counterstained with DAPI (blue) in all panels. The scale bar is $10 \mathrm{~mm}$ for all panels except panel D on which it is $20 \mathrm{~mm}$. $185 \times 146 \mathrm{~mm}(150 \times 150 \mathrm{DPI})$ 
Page 17 of 17

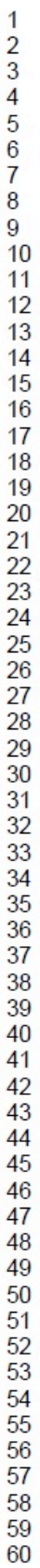

British Journal of Dermatology
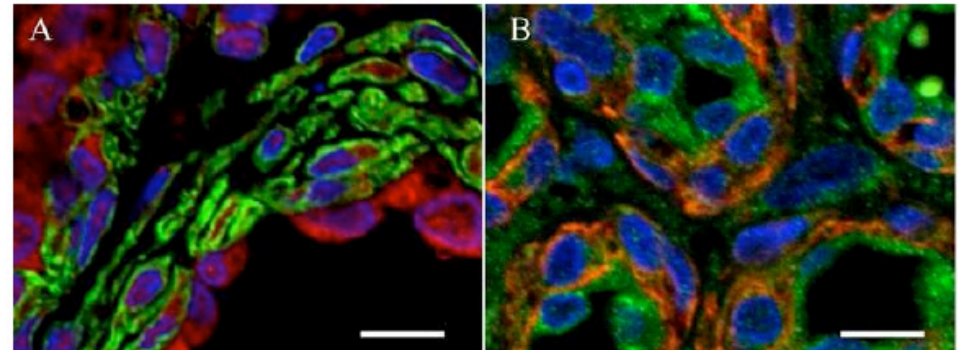

Figure 2

The pericyte layer in proliferating IH does not express Oct-4 or SSEA-4. Panels A\&B show representative IHC staining of proliferating IH in which the pericyte layer is identified by IR for smooth muscle actin (SMA, green staining). Panel A shows that the SMA+ pericyte layer (qreen staining) shows minimal IR for Oct-4 (red staining) but the endothelium shows strong IR for Oct-4 consistent with the data presented in Figure 1, Panel A. Similarly, the IR for SSEA-4 is greatest on the endothelium with only weak staining being detected on the SMA+ (red staining) pericyte layer. Cell nuclei are counterstained with DAPI (blue) in both panels. Scale bars are $10 \mathrm{~mm}$. $122 \times 52 \mathrm{~mm}(150 \times 150 \mathrm{DPI})$ 


\section{Chapter 10: Placental Chorionic Villous Mesenchymal Core Cells and Infantile Haemangioma}

\subsection{Introduction and Overview}

Chapter 9 demonstrates the expression of hESC markers on two distinct populations of cells, suggesting a possible cellular origin downstream of hESC. This knowledge coupled with previous reports highlighting the similarities in protein expression of proliferating $\mathrm{IH}$ and placenta $a^{6,84-86}$ pointed to the trophoblast ${ }^{87}$ as a possible cellular origin of this enigmatic condition.

This chapter demonstrates the expression of the trophoblastassociated proteins namely human placental lactogen $(\mathrm{hPL})^{88}$ and human chorionic gonadotrophin (hCG) ${ }^{88}$, but not human leucocyte antigen-G (HLA-G) or cytokeratin $7(\mathrm{CK} 7)^{89}$ on the haemogenic endothelium layer of proliferating $\mathrm{IH}$. These observations confirm that trophoblasts are possibly not the origin of $\mathrm{IH}$, which in turn focused our attention on the mesenchymal core cells of the placental chorionic villi. These cells, which are derived from extra-embryonic primitive streak have been reported to express $\mathrm{hCG}^{90}$ and $\mathrm{hPL}$ but not HLA-G or $\mathrm{CK}^{91}$, similar to the expression profile of proliferating IH endothelium. In support of this placental chorionic mesenchymal core cellular origin of $\mathrm{IH}$, is the ability for these cells to undergo vasculogenesis and the formation of a haemogenic endothelium ${ }^{92}$, consistent with the findings presented in Chapters 3 to 5 . This is fascinating as it may explain the higher incidence of these 
tumours in infants that have undergone in utero procedures, such as aminiocentesis ${ }^{93}$ or chorionic villous sampling ${ }^{93}$. This observation suggests that trauma associated with in utero samplings results in shedding of the placental chorionic villous mesenchymal core cells and is consistent with previously described similarities in protein expression between $\mathrm{IH}$ and the placenta.

\subsection{Contribution}

The novel concept of the placental chorionic mesenchymal core cell origin of $\mathrm{IH}$ was conceived by myself. The initial IHC experiments and the draft for the manuscript was written by myself and edited by my supervisors. Cofirmation of the expression of the placental by repeating the $\mathrm{IHC}$ experiements on other IH specimens were performed by C Tan \& S Guthrie.

\subsection{Manuscript: A Placental Chorionic Villous Mesenchymal}




\section{A placental chorionic villous mesenchymal core cellular origin for infantile haemangioma}

Tinte Itinteang, ${ }^{1,2,3}$ Swee T Tan, ${ }^{1,2,3,4}$ Siobhan Guthrie, ${ }^{1}$ Cherise E S Tan, ${ }^{3}$ Ben C Mclntyre, ${ }^{2,3}$ Helen D Brasch, ${ }^{2,3,5}$ Darren J Day ${ }^{1,2,3}$

'School of Bialogical Sciences, Victoria University of Wellington, Wellington, New Zealand ${ }^{2}$ Centre for the Study an
Treatment of Vascular Treatment of Vascular
Birthmarks, Wellington Regional Plastic, Maxillofacial and Burns Unit, Hutt Hospital, Wellington, New Zealand

${ }^{3}$ Gillies Mclndoe Research

Institute, Wellington, New

Zealand

${ }^{4}$ Wellington School of Medicin

and Heath Sciences, University

and Health Sciences, University

of Otago, Wellingtan, New

Zealan

DDepartment of Pathology, Hutt

Hospital, Wellington, New

Zealand

Correspondence to

Professar Swee T Tan,

Wellington Regianal Plastic

Maxillofacial and Bums Unit

Hutt Hospital, High Stret,

Hutt Hospital, High Street,

Nivate Bag 31-907, Lower

New Zealand; swee.
huttvalleydhb.org.nz

Swee T Tan and Darren J Day are equal senior authors.

Accepted 8 June 2011

\section{ABSTRACT}

Aims To investigate the expression of the placental cellspecific associated proteins in infantile haemangioma (IH).

Methods Immunohistochemical staining was used to investigate the expression of human chorionic gonadotrophin (hCG), human placental lactogen (hPL). human leucocyte antigen-G (HLA-G), cytokeratin 7 (CK7) and smooth muscle actin in paraffin-embedded sections of proliferating and involuted IHs.

Results The proteins hCG and hPL were expressed by the endothelium but not the pericyte layer of proliferating $\mathrm{IH}$, but these proteins were not detected in involuted lesions. There was no expression of CK7 and HLA-G in $\mathrm{IH}$.

Conclusions The expression of hCG and hPL, but not CK7 or HLA-G, by the endothelium of proliferating $\mathbb{H}$ supports a placental chorionic villous mesenchymal core cellular origin for $\mathrm{IH}$ rather than a trophoblast origin.

\section{INTRODUCTION}

Infantile haemangioma $(\mathrm{IH})$, the most common tumour of infancy, affects $10 \%$ of Caucasian infants with preponderance in white, female and premature infants. ${ }^{1-4} \mathrm{IH}$ typically undergoes rapid proliferation during infancy followed by slow spontaneous involution over the next 5-10 years, often leaving a fibro-fatty residuum. ${ }^{1-5}$

$\mathrm{IH}$ is considered a primary tumour of the microvasculature; however, recent data support a primitive mesoderm derived haemogenic endothelium phenotype for the microvessels of proliferating $\mathrm{IH}^{67}$ This is further substantiated by the observation that cells from proliferating $\mathrm{IH}$ have the ability to undergo erythropoietic ${ }^{10}$ and mesenchymal $^{11}$ differentiation. The demonstration of embryonic stem cell associated proteins in proliferating $\mathrm{IH}$ suggests that this lesion contains cells with an early embryonic origin, ${ }^{12}$ and is more consistent with the notion that $\mathrm{IH}$ is a developmental anomaly rather than simply an aberrant proliferation of immature endothelial cells.

Consistent with this, and in support of the suggestion that $\mathrm{IH}$ has a placental origin, is the recent report by our laboratory that the endothelium of proliferating $\mathrm{IH}$ expresses embryonic haemoglobin $\zeta{ }^{10}$ This haemoglobin chain is only expressed during the haematopoetic stage of erythropoiesis that occurs exclusively during the first trimester. ${ }^{30}$ Additionally, a unique microvascular phenotype, which includes the expression of GLUT- $1,{ }^{5}$ is shared by $\mathrm{IH}$ and human placenta ${ }^{15}$ and has led to the proposed placental embolic origin of $\mathrm{IH}^{5}{ }^{15}$ The unique co-expression of proteins common to placenta and $\mathrm{IH}$ has been further supported by mRNA expression studies. ${ }^{14-16}$ In 2001 Bree et $a l^{17}$ speculated a placental trophoblastic origin for $\mathrm{IH}$, although they failed to demonstrate the expression of trophoblast markers in this condition. Clinically, a placental origin is supported by the observation of an increased incidence of $\mathrm{IH}$ following chorionic villous sampling and, to a lesser extent, amniocentesis. ${ }^{13}$

A human placenta consists of two distinct populations of cells derived from two different stem/progenitor cell populations. Trophoblasts are established at the blastocyst stage and are the first cells to undergo differentiation from the totipotent blastomeres. ${ }^{18} 19$ They are specialised embryonic epithelial cells constituting the trophoectoderm, which gives rise to the mural and the polar trophoectodermal layers. ${ }^{18} 20$ The trophoblasts of the polar layer ultimately give rise to the synctiotrophoblasts and trophoblast giant cells of the placenta, and these are responsible for the invasion of and absorption of nutrients from the maternal uterine circulation. ${ }^{18} 20 \quad 21$ Trophoblasts can be identified by the combined expression of human chorionic gonadotrophin (hCG), human placental lactogen (hPL), cytokeratin 7 (CK7) and human leucocyte antigen-G (HLA-G) ${ }^{22-25}$ Trophoblasts ultimately give rise to the cells forming the outer layer of the placental chorionic villi. In contrast the inner mesenchymal core of the placental villi is formed from the extraembryonic primitive mesoderm that arises from the primitive streak located at the caudal end of the embryonic plate. This extraembryonic primitive mesoderm also gives rise to the mesodermal layer of the yolk sac, amnion, placental chorion and the body stalk. ${ }^{26}$ The inner mesenchymal core cells of the placental chorionic villi are responsible for the de novo vasculogenesis and have been shown to express $\mathrm{hCG}^{27}$ and the mesenchymal marker vimentin, ${ }^{29}$ but not $\mathrm{CK} 7$ or HLA-G. These observations have led us, in this report, to investigate the expression of the placenta-associated proteins $\mathrm{hCG}, \mathrm{hPL}, \mathrm{CK} 7 \mathrm{and}$ HLA-G in IH in an attempt to clarify the cellular origin of $\mathrm{IH}$. We have previously demonstrated that the endothelium of $\mathrm{IH}$ expresses vimentin. ${ }^{11}$

\section{METHODS}

\section{Immunohistochemistry}

Paraffin sections $(4 \mu \mathrm{m})$ of proliferating $\mathrm{IH}$ from 12 patients (age 3-10 months; mean, 6 months), and involuted lesions from three patients (age 8,9 and 11 years) were obtained according to a protocol approved by the Wellington Regional Ethics 
Original article

Committee. All primary antibodies used for immunolabelling were purchased from Abcam (Cambridge, Massachusetts, USA), and were used diluted as indicated: CD34 (1:200), GLUT-1 (1:300), hPL (1:300), hCG (1:300), HLA-G (1:300), CK7 (1:300) and smooth muscle actin (SMA; 1:400). Immunolabelling was performed as previously described. ${ }^{6-10}$ Bound antibodies for $\mathrm{hPL}$, hCG, HLA-G and CK7 were detected using a digoxigeninrhodamine tertiary cascade amplification, as previously described. $^{78}$ Term human placental tissue and normal subcutaneous fat from two adult patients were used as controls to confirm antibody specificity.

\section{RESULTS}

\section{Immunohistochemistry}

The microvessels of proliferating $\mathrm{IH}$ are characterised by an inner endothelial layer expressing CD34 and an outer concentric layer showing immunoreactivity (IR) for SMA. ${ }^{7}$ The expression of GLUT-1, the marker for $\mathrm{IH}^{5}$ by the endothelial layer was demonstrated in all $\mathrm{IH}$ lesions studied (figure $1 \mathrm{~A}$, red) and was distinct from the outer concentric pericyte layer showing IR for SMA (figure $1 \mathrm{~A}$, green).

Figure $1 \mathrm{~A}-\mathrm{C}$ shows that the cells of the endothelium of $\mathrm{IH}$, identified by IR for CD34 (green staining), also show IR for hCG (red staining, B) and hPL (red staining, C). To confirm that the IR for hCG and hPL was localised to the capillary endothelium rather than the concentric outer pericyte layer, staining for SMA was undertaken in conjunction with that for hCG and hPL. Figure $1 \mathrm{D}, \mathrm{E}$ shows that IR for SMA (green staining) is localised to the pericyte layer, and that the pericytes show weak IR for hCG and hPL (red staining in D and $\mathrm{E}$, respectively). In contrast, figure $1 \mathrm{~F}$ shows that the $\mathrm{CD} 34+$ endothelium (green staining) does not show IR for CK7. Similarly, figure 1G shows minimal IR for HLA-G (red staining) with the pericyte layer being identified by IR for SMA (green staining). In contrast to the IR for hCG and hPL detected on proliferating lesions (figure $1 \mathrm{~B}, \mathrm{C}$ ), minimal IR was found in similarly processed involuted $\mathrm{IH}$ lesions. Figure $1 \mathrm{H}, \mathrm{I}$ shows IR for hCG and hPL, respectively, in a representative involuted lesion (red staining). The capillary endothelium is identified in figure $1 \mathrm{H}, \mathrm{I}$ by IR for CD34 (green
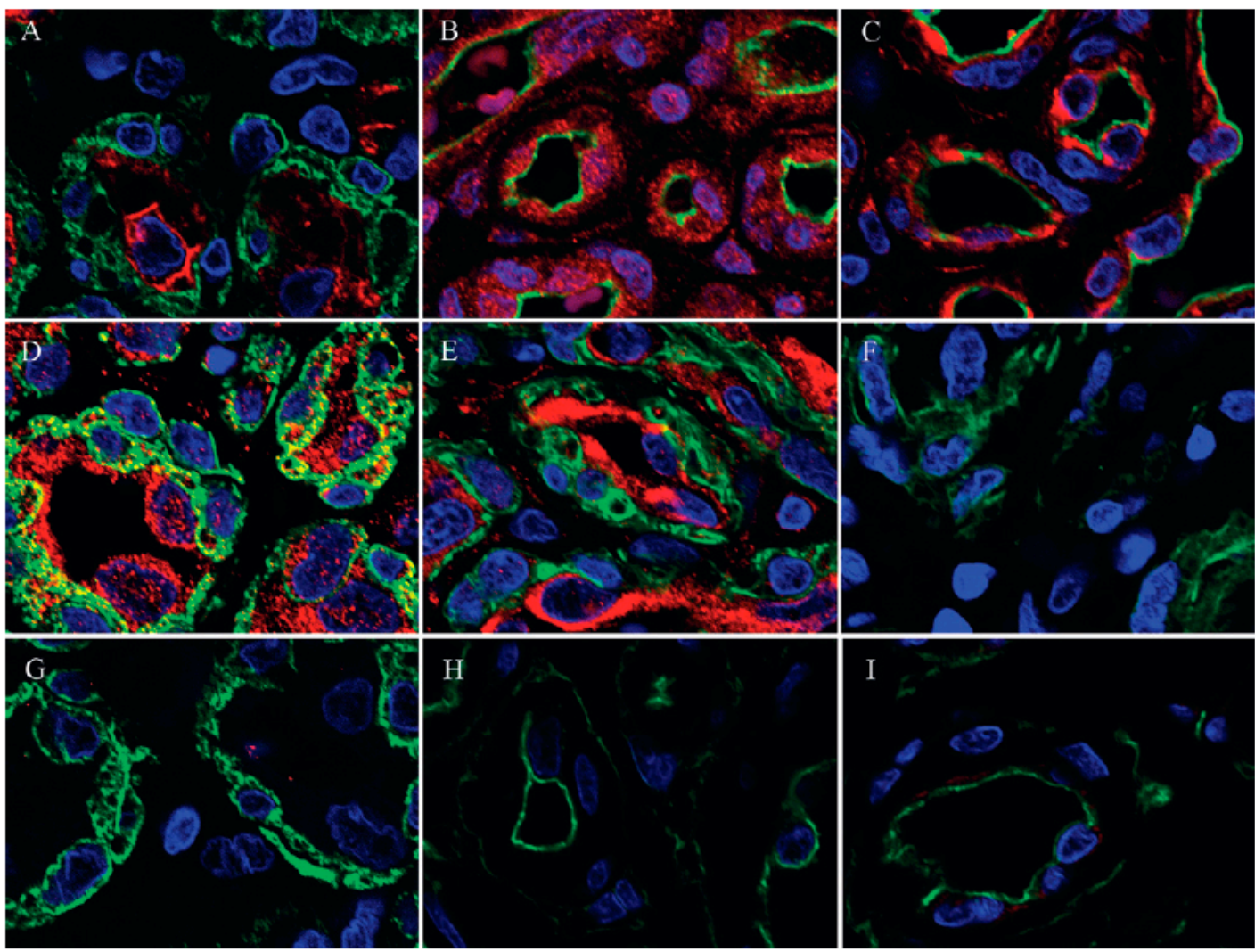

Figure 1 Immunohistochemical staining of infantile haemangioma for placental-associated proteins. (A-G) Staining of representative proliferating lesions, and $(\mathrm{H}, \mathrm{I})$ involuted lesions. (A) The endothelium of the microvessels shows immunoreactivity (IR) for GLUT-1 (red staining) that is distinct from the outer concentric pericyte layer which shows IR for smooth muscle actin (SMA; green staining). (B) Human chorionic gonadotrophin (hCG; red staining) and (C) human placental lactogen (hPL; red staining) IR is located on the CD34+ (green staining) cells that form the endothelium. (D) hCG (red staining) and (E) hPL (red staining) are not expressed by the pericyte layer that shows IR for SMA (green staining). (F) IR for cytokeratin 7 and (G) human leucocyte antigen-G (HLA-G; red staining respectively) were not observed on the endothelium of the microvessels. Pericytes were identified by IR for SMA (green staining). All sections are counterstained with 4',6-diamidino-2-phenylindole (DAPI; blue staining). 
staining). Similar to the proliferating lesions, no IR for CY7 or HLA-G was observed for the involuted lesions (data not shown). To confirm the specificity of the IHC staining, the same antibodies were used to stain term placental tissue as a positive control. Figure 2 shows IR for hCG (A, red staining), hPL (B, red staining), CK7 (C, red staining) and HLA-G (D, red staining). IR for all the above markers is clearly visible on the outer epithelia layer of the placental chorionic villi, while the endothelium of the villi is identified by IR for CD34 (A-D, green staining). A expected, adult subcutaneous fat does not show IR for either hCC or $\mathrm{hPL}$ (red staining in $\mathrm{E}$ and $\mathrm{F}$, respectively), but IR for CD34 on the endothelium of blood vessels (green staining) can be seen.

\section{DISCUSSION}

The demonstration that $\mathrm{IH}$ is a developmental anomaly involving proliferation of a haemogenic endothelium ${ }^{6}$ derived from a primitive mesoderm/mesenchymal phenotype ${ }^{71}$ represents a paradigm shift in the understanding of this enigmatic condition. Our previous demonstration of expression of haemoglobin $\zeta$ and the erythropoietin receptor by the cells forming the capillary endothelium is consistent with our current report demonstrating expression of placental-specific proteins, and our report showing that explanted proliferating $\mathrm{IH}$ tissue can undergo primitive haematopoiesis when cultured in vitro. ${ }^{10}$

The observation of the unique co-expression of GLUT-1, FcrRII, Lewis $\mathrm{Y}$ antigen and merosin in both $\mathrm{IH}$ and placental chorionic villi, ${ }^{5}{ }^{15}$ and the expression of the type 3 iodothyronine deiodinase enzyme by placental tissue ${ }^{31}$ in $\mathrm{IH}^{14}$ has led us to reexamine the suggestion that $\mathrm{IH}$ may be derived from the placenta. ${ }^{17}$ Furthermore, our recent report of the expression of embryonic haemoglobin chain $\zeta$ and primitive haematopoiesis in proliferating $\mathrm{IH}^{10}$ is consistent with the same processes also taking place within the first trimester placental tissues. ${ }^{32}$
The expression of placenta-associated proteins has provided a unique identification signature of placental trophoblasts. hCG a hormone secreted by trophoblasts, with the exception of cytotrophoblasts, ${ }^{22}$ is responsible for maintaining progesterone release from corpus luteum. ${ }^{33} \mathrm{hPL}$ is also expressed by trophoblasts, with the exception of cytotropholasts, ${ }^{22}$ and is responsible for the growth and development of the fetus during gestation. ${ }^{34}$ Likewise CK7 is a keratin filament type expressed in all trophoblast subpopulations, ${ }^{24}$ and HLA-G is a major histocompatibility gene expressed in the placenta throughout the
three trimesters of pregnancy by trophoblast lineages.

As far as we are aware, this is the first study demonstrating the expression of hCG and hPL, but not CK7 or HLA-G, by the endothelium of the capillaries of proliferating $\mathrm{IH}$, and that these markers are lost as $\mathrm{IH}$ involutes. Bree et al ${ }^{17}$ hypothesised that placental trophoblasts are the precursor cells for $\mathrm{IH}$, but failed to demonstrate the expression of the trophoblast markers hCG and $\mathrm{hPL}$, as we have been able to do in this study. This difference is most likely attributable to better antibodies being available for our study and the use of a tertiary amplification cascade with fluorescent detection for the IHC analysis. We were, however, able to corroborate the finding of Bree et al in that we were also unable to demonstrate IR for CK7 or HLA-G in IH. This is intriguing given that placental trophoblasts also express these proteins. $^{25} 37$ This observation focused our attention to the placental chorionic villous mesenchymal core cells being the possible origin of $\mathrm{IH}$.

The placental chorionic villi are formed from two different populations of cells, with the epithelial layer derived from trophoblasts and the inner mesenchymal core derived from the extraembryonic primitive mesoderm. The placental chorionic villous mesenchymal core cells have been shown to express $\mathrm{hCG}^{27}$ and $\mathrm{hPL},{ }^{28}$ but not CK7 or HLA-G. ${ }^{24} 25$ This observation is supported by reports demonstrating that these mesenchymal
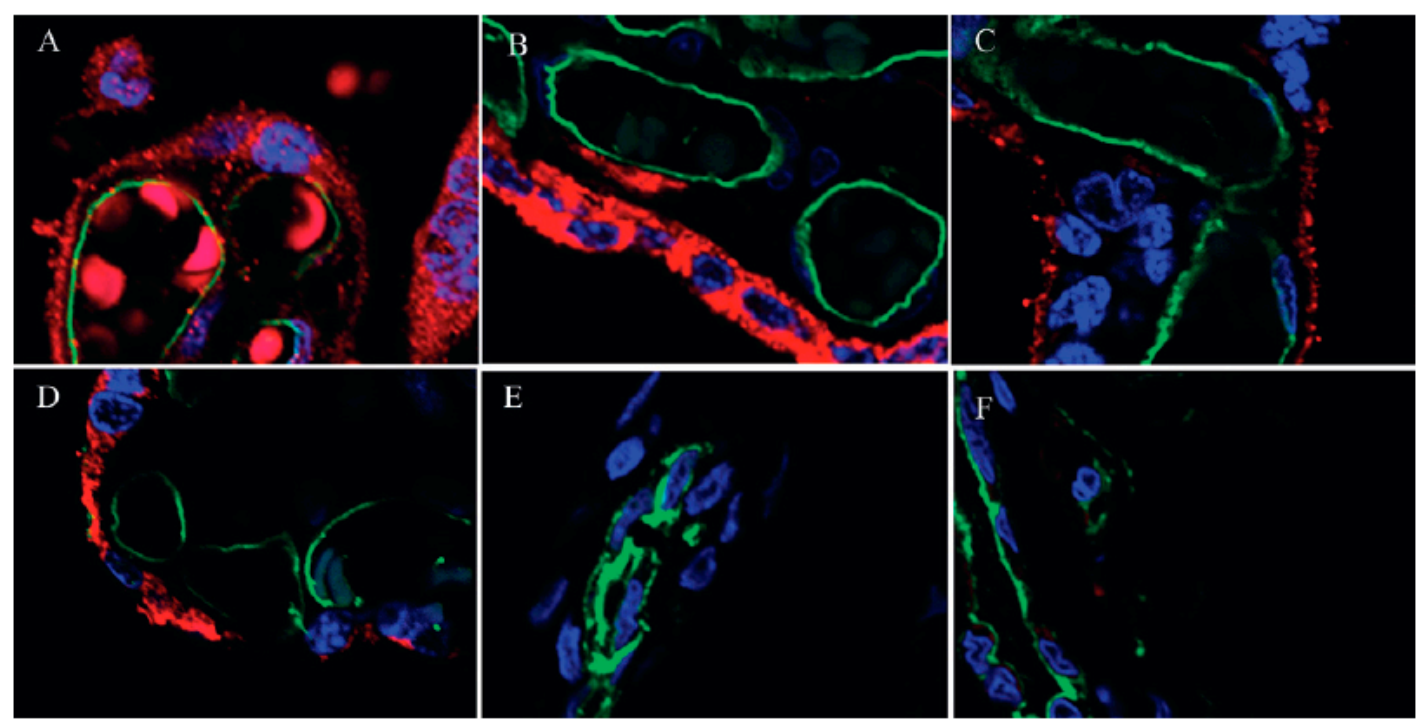

Figure 2 Immunohistochemical staining on a term placenta for placenta-associated proteins. (A-D) Staining for human chorionic gonadotrophin (hCG), human placental lactogen (hPL), cytokeratin 7 and human leucocyte antigen-G, respectively (red staining). The endothelium of the placental chorionic villi is identified by immunoreactivity (IR) for CD34 (green staining). (E,F) Adult subcutaneous fat shows no IR for hCG or hPL (red staining) but some blood vessels show IR for CD34 (green staining). All sections are counterstained with 4',6-diamidino-2-phenylindole (DAPI, blue staining). 


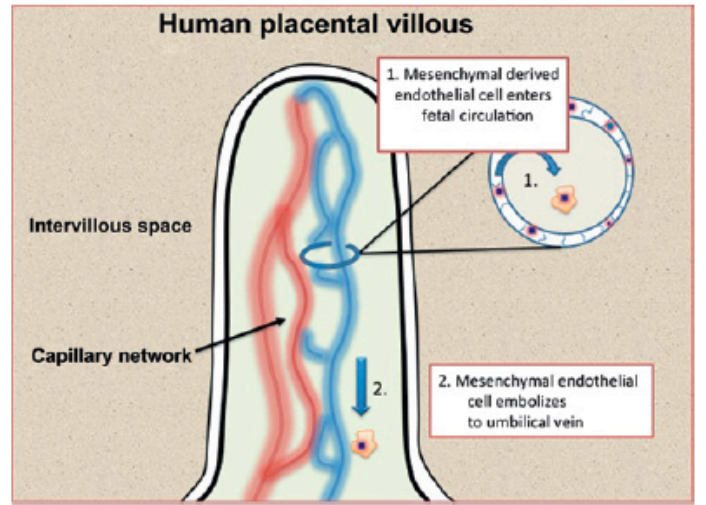

Figure 3 Schema showing a possible placental chorionic villous mesenchymal core cellular origin of $\mathrm{IH}$. Shedding of the human placenta lactogen positive and human chorionic gonadotrophin positive but cytokeratin 7 negative and human leucocyte antigen-G negative primitive mesoderm derived cells from the primitive capillaries of the placental chorionic villous core into the fetal circulation may occur spontaneously during early pregnancy or following obstetric interventions. The postnatal extrauterine environment with high levels of plasma renin during infancy and early childhood provides an environment for the development of infantile haemangioma.

core cells are derived from extraembryonic primitive mesoderm that is able to undergo de novo vasculogenesisvia the formation of a haemogenic endothelium and blood islands. ${ }^{6737}$ This is further substantiated by the mesenchymal staining patterns reported for first trimester placental chorionic villous mesenchymal core cells that show IR for viment in and CD $34 .^{29}$ These properties are strikingly similar to our previous reports that $\mathrm{IH}$ has a primitive mesodermal/mesenchymal phenotype ${ }^{711}$ with a haemogenic capability. ${ }^{610}$ Collectively, these data are consistent with $\mathrm{IH}$ being derived from placental chorionic villous mesenchymal core cells rather than from trophoblasts.

Our results, along with the observation of increased incidence of $\mathrm{IH}$ in pre-eclampsia, ${ }^{38}$ or following chorionic villous sampling or amniocentesis, ${ }^{13}$ suggest that shedding of primitive mesoderm derived cells from the primitive capillaries of the placental chorionic villi, via the umbilical vein, into the fetal circulation (figure 3 ) occurs spontaneously during early pregnancy or following the obstetric interventions. The extrauterine environment, with high levels of plasma renin during infancy and early childhood, may then provide an environment for the development of $\mathrm{IH}^{8}{ }^{8}$ This hypothesis is consistent with idea that the renin-angiotensin system regulates $\mathrm{IH}$ progression. ${ }^{8}$

An adequate explanation of why some $\mathrm{IH}$ are segmental and others present as discrete foci has been lacking. The identification of $\mathrm{IH}$ being derived from the inner mesenchymal core cells of the placental chorionic villi, along with the increased incidence of $\mathrm{IH}$ being associated with placental trauma, have led us to hypothesise that embolisation of placental chorionic villous mesenchymal core cells prior to the neural crest somite migration stage of fetal development may result in integration of these cells into the migrating neural crest cell wave, resulting in the segmental distribution of $\mathrm{IH}$, some of which constitute PHACE syndrome. ${ }^{39} 40$ Embolisation of these cells into the fetal circulation later in pregnancy may result in discrete lesion(s).

The results of our study suggest the critical involvement of placental chorionic villous mesenchymal core cells in the aeti-

\section{Take-home messages}

- Our recent data have shown a primitive mesoderm derived haemogenic endothelium phenotype for the microvessels of proliferating infantile haemangioma $(\mathrm{IH})$.

- Our observation that cells from proliferating $\mathrm{IH}$ have the ability to undergo erythropoietic and mesenchymal differentiation, and the demonstration of embryonic stem cell associated proteins in proliferating $\mathrm{IH}$, suggest that this lesion contains cells with an early embryonic origin, supporting the notion that $\mathrm{IH}$ is a developmental anomaly.

- The expression of human chorionic gonadotrophin and human placental lactogen, but not cytokeratin 7 or human leucocyte antigen-G, by the endothelium of proliferating IH supports a placental chorionic villous mesenchymal core cellular origin for IH.

ology of $\mathrm{IH}$, and they explain previous observations of the unique similarities between $\mathrm{IH}$ and placental tissue. Additionally, we propose a rational explanation to account for the origin of segmental and focal $\mathrm{IH}$.

Acknowledgements We thank the laboratory staff at the Department of Pathology. Hutt Hospital, for their assistance in processing of the paraffin sections used in this study.

Funding This wark is supported by grants from the Gillies Mclndoe Foundation, Wellington Regional Plastic Surgery Unit Research and Education Trust, and the Victoria University of Wellington Research Trust. Dr Itinteang is supported by the Royal Australasian College of Surgeons' Foundation for Surgery Scholarship.

Competing interests None.

Ethics approval Ethics approval was obtained from Wellington Regional Ethics Committee.

Provenance and peer review Not commissioned; externally peer reviewed.

\section{REFERENCES}

1. Tan ST, Velickovic M, Rüger BM, et al. Cellular and extracellular markers of hemangioma. Plast Aeconstr Surg 2000;106:529-37.

2. Tan ST, Itinteang T, Leadbitter P. Low dose propranolol for multiple infantile hepatic and cutaneous hemangiomas with deranged liver function. Pediatics 2011;127:772-6.

3. Tan ST, Itinteang T, Leadbitter P. Low-dose propranolol for infantile haemangioma. $J$ Plast Reconstr Aesth Surg 2011;64:292-9.

4. Itinteang $\mathbf{T}$, Withers AHJ, Leadbitter $\mathrm{P}$, et al. Pharmacologic therapies for infantile hemangioma -is there a rational basis? Plast Reconstr Surg 2011 (accepted).

5. North PE, Waner M, Mizeracki A, et al. Glut 1: a newly discovered immunohistochemical marker for juvenile hemangiomas. Hum Patho 2000;31:11-22.

6. Itinteang $\mathbf{I}$, Tan ST, Brasch $\mathrm{H}$, et al. Haemogenic endothelium in infantile haemangioma. J Clin Pathol 2010;63:982-6.

7. Itinteang $\mathbf{I}$, Tan $\mathrm{ST}$, Brasch $\mathrm{H}$, et al. Primitive mesodermal cells with a neural crest stem cell phenotype predominate prolfferating infantile haemangioma. $J$ Clin Pathol 2010;63:771-6.

8. Itinteang $\mathbf{T}$, Brasch HD, Tan ST, et al. Expression of components of the rennin-angiotensin system in prolferating infantile haemangioma may account for the propranolol-induced accelerated involution. $J$ Plast Reconst Aesth Surg 2011;64:759-65.

9. Vishvanath A, Itinteang T, Tan ST, et al. Infantile haemangioma expresses TRAL TRAIL receptors, osteoprotegerin and RANKL. Histopsthology 2011 (accepted).

10. Itinteang $\mathrm{I}$, Tan ST, Brasch $H D$, et al. Primitive erythropoiesis in infantile Itinteang I, Tan ST, Brasch HD, et al. Primitive eny

11. Itinteang $\mathrm{T}$, Vishvanath A, Day DJ, et al. Mesenchymal stem cells in infantile Itinteang $\mathrm{I}$, Vishvanath $\mathrm{A}, \mathrm{Day} \mathrm{DJ}$, et al. Mese

. haennangioma. J Clin Pathol 2011;64:232-6. Itinteang T, Tan ST, Brasch HD, et al. Infantile hen

stem cell Tarker. Br Jermatol 2011 (submitted).

(1) incidence after chorionic villus sampling. Prenat Diagn 2010;30:913-17.

14. Huang SA, Tu HM, Harney JW, et al. Severe hypothyroidism caused by type 3 iodothyronine deiodinase in infantile hemangiomas. $N$ Eng/ J Med 2000;343:185-9 North PE, Waner M, Mizeracki A, et al. A unique microvascular phenotype shared
juvenile hemangiomas and human placenta. Arch Dermato/ 2001;137:559-70. 
16. Barnes CM, Huang S, Kaipainen A, et al. Evidence by molecular proffing for a placental origin of infantile hemangioma. Proc Nat Acad Sci U S A 2005; 102:19097-102

17. Bree AF, Siegried E, Sotelo-Avila C, et al. Infantile hemangiomas: speculation on placental trophoblastic origin. Arch Dermatol 2001:137:573-7.

18. Cross JC, Werb Z, Fisher SJ. Implantation and the placenta: key pieces of the development puzzle. Science 1994:266:1508-18

19. Knöfler M, Vasicek R, Schreiber M. Key regulatory transcription factors involved in placental trophoblast development-a review. Placenta 2001;22 Suppl A:S83-92.

20. Cross JC. Genetic insights into trophoblast differentiation and placental morphogenesis. Semin Cell Dev Biol 2000;11:105-13.

Scifres CM. Nelson DM. Intrauterine growth restriction, human placenta development and trophoblast cell death. J Physiol 2009; 587:3453-8.

2. Kurman RJ, Young RH, Noris HJ, et al. Immunochemical localisation of placental lactogen and chorionic gonadotrophin in the normal placenta and trophoblastic tumours, with emphasis on intermediate trophoblast and the placental site trophoblastic tumour. Int $J$ Gynecol Pathol 1984;3:101-21.

23. Jones $\mathbf{C J}$, Fox $\mathrm{H}$. Uttrastructure of the normal human placenta. Electron Microsc Rev $1991 ; 4: 129-78$.

24. Blaschitz A. Weiss U, Dohr G, et al. Antibody reaction patterns in first trimester placenta: implications for trophoblast isolation and purity screening. Placenta 2000;21:733-41.

25. Goldman-Wohl DS, Ariel I, Greenfield C, et al. HLA.G expression extravillous trophoblasts is an intrinsic property of cell differentiation: a lesson learned from trophoblasts is an intrinsic property of ceil differentiation:

26. Luckett WP. Origin and differentiation of the yolk sac and extraembryonic Luckete W. Origin and difterentation of the yolk sac and extraembryonic 1978:152:59-98.

27. Phillips CN, McCue PA, Whitsett CF, et al. Differentiation in human chorionic villous cultures: hcg and HLA expression. Prenat Diagn 1989;9:227-42.

28. Hempstock J, Cindrova-Davies $T$, launiaux $E$, et al. Endometrial glands as a source Hempstock J, Gindrova-Davies $\mathrm{T}$, Jauniaux $\mathrm{E}$, et al. Endometrial glands as a s
of nutrients, growth factors and cytokines during the first trimester of human of nutrients, growth factors and cytokines during the first trimester of human pregnancy.
29. Hartmann M, Blaschitz A, Hammer A, et al. Immunohistochemical examination of trophoblast populations in human first trimester and term placentae and of first trimester spiral arteries with the monoclonal antibody GZ112. Placenta 1997;18:481-9.

30. Schechter AN. Haemoglobin research and the origins of molecular medicine. Blood 2008; 112:3927-38

31. Huang SA, Dorfman DM, Genest DR, et al. Type 3 iodothyronine deiodinase is highly expressed in the human uteroplacental unit and in fetal epithelum. J Clin Endocrino Metab 2003;88:1384-8.

32. Soleymanlou N, Jurisica I, Nevo 0, et al. Molecular evidence of placental hypoxia in preeclampsia. J Clin Endocrinol Metab 2005:90:4299-308.

3. Norwitz ER. Schust DJ, Fisher SI. Implantation and survinal of early pregnancy. $N$ Engl J Med 2001:345:1400-8.

34. Handwerger S, Freemark M. The roles of placental growth hormone and placental lactogn in the regulation of human fetal growth and development. $J$ Pediatr Endocrinol Metab 2000;13:343-56.

35. Maldonado-Estrada J, Menu $E$, Roques $P$ et al. Evaluation of cytokeratin 7 aldonado-Estrada J, Menu $E$, Alques $P$ et al, Evalus the purity of hum as an 2004:286:21-34.

36. Ema M, Yokomizo T, Wakamatsu A, et al. Primitive erythropoiesis from mesoderma precursors expressing VE

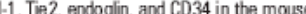
08:4018-24

37. Haggstrom AN, Drolet BA, Baselga E, et al. Prospective study of infantile Haggstrom AN, Drolet BA, Baselga E, et al. Prospective study of infantile hemangiomas. de $2007 \cdot 150 \cdot 291-4$.

38. Waner $\mathrm{M}$. North PE, Scherer KA, et al. The nonrandom distribution of facial Waner M, North PE, Scherer KA, et al. The non

39. Metry DW. Hawrot A, Altman C, et al. Association of solitary, segmenta Metry DW, Hawrot A, Aitman C, et al. Association of solitary, segment 2004:140:591 -6.

40. Rhodes KE, Gekas C, Wang Y, et al. The emergence of hematopoietic stem cells is intiated in the placental vasculature in the absence of circulation. Cell Stem Cell $2008 \cdot 2 \cdot 252-63$ 


\section{Chapter 11: Treatment of Infantile Haemangioma}

\subsection{Introduction and Overview}

There is a need for intervention in about $10 \%$ of $\mathrm{IHs}$, as there is an inherent risk to the infant's life or function due to either tissue distortion or destruction. The mainstay of intervention for problematic proliferating $\mathrm{IH}$ is pharmacological therapy, High-dose corticosteroids being the first line of therapy, until the serendipitous discovery of the dramatic effect of propranolol in this condition ${ }^{25}$. Propranolol has become the preferred treatment for this condition $^{27,94-97}$

In this chapter I describe our research group's experience with the use of propranolol in this group of patients, and further to this, we attempt to explain the observed effect of this pharmacologic agent with regard to the results presented in earlier chapters. In particular I attempt to identify critical pathways governing the stem cell biology of these tumours in an attempt to identify safer alternatives to this particularly 'vulnerable' population affected by this condition. These findings have led to a trial in the use of captopril, an ACE inhibitor, and the effect of this novel use of this medication is also reported.

\subsection{Contribution}


My involvement in the clinical reports involved analysis and editing of the manuscripts, whereas for the review paper the manuscript was drafted by myself and edited by my supervisors.

\title{
11.3. Manuscript: Low-Dose Propranolol For Infantile
}

\author{
Haemangioma
}




\title{
Low-dose propranolol for infantile haemangioma ${ }^{\text {i⿱ }}$
}

\author{
Swee T. Tan ${ }^{\text {a,b,c,*}}$, Tinte Itinteang ${ }^{\text {a,d }}$, Philip Leadbitter ${ }^{\text {a,e }}$
}

${ }^{a}$ Centre for the Study \& Treatment of Vascular Birthmarks, Wellington Regional Plastic, Maxillofacial \& Burns Unit, Hutt Hospital, Wellington, New Zealand

${ }^{\mathrm{b}}$ Gillies McIndoe Research Institute, Wellington, New Zealand

c University of Otago; New Zealand

'School of Biological Sciences, Victoria University of Wellington, Wellington, New Zealand

e Department of Paediatrics, Hutt Hospital, Wellington, New Zealand

\author{
KEYWORDS \\ Haemangioma; \\ Involution; \\ Propranolol; \\ Renin-angiotensin \\ system; \\ Angiotensin II; \\ Angiotensin-converting \\ enzyme
}

Summary In 2008, propranolol was serendipitously observed to cause accelerated involution of infantile haemangioma. However, the mechanism by which it causes this dramatic effect is unknown, the dosage empirical and the optimal duration of treatment unexplored. This study determines the minimal dosage and duration of propranolol treatment to achieve accelerated involution of problematic infantile haemangioma.

Consecutive patients with problematic proliferating infantile haemangioma treated with propranolol were culled from our prospective vascular anomalies database. The patients were initially managed as inpatients and commenced on propranolol at $0.25 \mathrm{mg} \mathrm{kg}^{-1}$ twice daily, and closely monitored. The dosage was increased to $0.5 \mathrm{mg} \mathrm{kg}^{-1}$ twice daily after $24 \mathrm{~h}$, if there was no cardiovascular or metabolic side effect. The dosage was increased further by $0.5 \mathrm{mg} \mathrm{kg}^{-1}$ day ${ }^{-1}$ until a visible effect was noticed or up to a maximum of $2 \mathrm{mg} \mathrm{kg}^{-1} \mathrm{day}^{-1}$, and was maintained until the lesion had fully involuted or the child was 12-months old.

A total of 15 patients aged 3 weeks to 8.5 months (mean, 11 weeks) underwent propranolol treatment for problematic proliferating infantile haemangioma, which threatened life $(n=1)$ or vision $(n=2)$ or nasal obstruction $(n=3)$ and/or caused ulceration $(n=6)$ and/or bleeding $(n=2)$ and/or significant tissue distortion $(n=12)$. The minimal dosage required to achieve accelerated involution was $1.5-2.0 \mathrm{mg} \mathrm{kg}^{-1} \mathrm{day}^{-1}$. Rebound growth occurred in the first patient when the dose was withdrawn at 7.5 months of age requiring reinstitution of treatment. No rebound growth was observed in the remaining patients. No other complications were observed.

Propranolol at $1.5-2.0 \mathrm{mg} \mathrm{kg}^{-1} \mathrm{day}^{-1}$, administered in divided doses with gradual increase in the dose, is effective and safe for treating problematic proliferating infantile haemangioma in

\footnotetext{
This article was presented, in part, at the Royal Australasian College of Surgeons' Annual Scientific Congress, Brisbane, Australia, 11-16 May 2009; the Australian and New Zealand Vascular Anomalies Interest Group Meeting, Melbourne, Australia, 22 October 2009; the New Zealand Association of Plastic Surgeons' Annual Scientific Meeting, Wellington, New Zealand, 21 November 2009; and the International Society for the Study of Vascular Anomalies Workshop, Brussels, Belgium, 21-24 April, 2010.

* Corresponding author. Wellington Regional Plastic, Maxillofacial \& Burns Unit, Hutt Hospital, High Street, Private Bag 31-907, Lower Hutt, Wellington, New Zealand. Tel.: +64 4587 2506; fax: +644587 2510.

E-mail address: swee.tan@huttvalleydhb.org.nz (S.T. Tan).
}

1748-6815/\$ - see front matter ( 2010 British Association of Plastic, Reconstructive and Aesthetic Surgeons. Published by Elsevier Ltd. All rights reserved. doi:10.1016/j.bjps.2010.06.010 
our cohort of patients. Treatment should be maintained until the lesion is completely involuted or the child is 12-months old. Larger scale studies confirming the safety and efficacy of propranolol may broaden the indications of treatment of proliferating infantile haemangioma.

(c) 2010 British Association of Plastic, Reconstructive and Aesthetic Surgeons. Published by Elsevier Ltd. All rights reserved.

Infantile haemangioma is the most common tumour of infancy with a preponderance in Caucasian, female and premature infants. ${ }^{1,2}$ It is characterised by rapid proliferation during infancy (proliferative phase), followed by slow but inevitable involution over the next $1-5$ years (involuting phase) with continued improvement up to 10 years (involuted phase), when the cellular elements are gradually replaced by fibro-fatty deposition. ${ }^{3,4}$

Approximately $10 \%$ of infantile haemangioma require intervention during infancy because the lesion poses a threat to life or function or causes tissue distortion or destruction. ${ }^{5,6}$ The mainstay treatment during the proliferative phase has been pharmacologic therapy with highdose steroid being the first-line treatment,,$^{2,7-9}$ since its serendipitous discovery to cause accelerated involution of infantile haemangioma in the 1960 s. $^{10,11} \mathrm{~A}$ dramatic response occurs in $30-55 \%$ of cases, equivocal results are seen in $40 \%$ and continued growth is observed in the remainder of the cases. ${ }^{8,12}$ The side effects of systemic steroid therapy in association with the treatment of problematic haemangioma are well documented. ${ }^{8,13}$

The second-line phamacologic treatment for problematic proliferating infantile haemangioma has been interferon with a $90 \%$ response rate. ${ }^{14}$ However, it is associated with significant adverse effects including spastic diplegia for up to $25 \%$ of patients. ${ }^{15,16}$ More recently, vincristine has become an alternative to interferon in steroid-resistant cases. ${ }^{17,18}$ Surgical excision remains a useful treatment in selected patients with problematic localised lesions $s^{2,19,20}$ and pulsed-dye laser has been used to expedite healing of ulceration..$^{5,19-21}$

Because there has been no effective treatment for infantile haemangioma, a conservative management approach has generally been adopted for proliferating lesions with surgery and/or laser therapy being used to address the residuum before the child goes to school. ${ }^{20}$

Propranolol was discovered serendipitously by LéatuéLabrèzeet al. ${ }^{22}$ in 2008 to induce accelerated involution of a large problematic facial proliferating haemangioma, when it was used to treat steroid-induced hypertophic cardiomyopathy. ${ }^{22}$ A limited number of case series have confirmed the dramatic effect of propranolol on proliferating infantile haemangioma. ${ }^{23-26}$

Although there has been a long and vast experience in the use of $\beta$-blockers in the paediatric population for tetralogy of Fallot $^{27}$ and hypertrophic obstructive cardiomyopathy, ${ }^{28}$ the experience with their use for infantile haemangioma is limited and concerns about their potential cardiovascular and metabolic side effects have been expressed. ${ }^{29}$ The mechanism of action of propranolol on infantile haemangioma is unknown and treatment remains empirical and the optimal dose and duration of treatment unexplored. This study documents our observation of the minimal dosage required to initiate accelerated involution of infantile haemangioma by propranolol and the optimal duration of treatment.

\section{Patients and methods}

Consecutive patients with problematic proliferating infantile haemangioma treated with propranolol at our Vascular Anomalies Centre were culled from our prospective vascular anomalies database 2008-2010.

The patients were routinely managed initially as inpatients and commenced on propranolol at $0.25 \mathrm{mg} \mathrm{kg}^{-1}$ orally twice daily. A 4-hourly heart rate, blood pressure and pre-prandial (30 $\mathrm{min}$ ) blood glucose level monitoring was performed. The patients were reviewed at $24 \mathrm{~h}$ for visible changes in the haemangioma and for any cardiovascular (e. g., hypotension) and metabolic side effects (e.g., hypoglycaemia). If the observations were stable, the dosage was increased by $0.5 \mathrm{mg} \mathrm{kg}^{-1}$ twice daily every $24 \mathrm{~h}$ until a response was observed, or up to $2 \mathrm{mg} \mathrm{kg}^{-1}$ day $^{-1}$.

The patients were routinely followed up in the outpatient's clinic within 1 week and the dose was further increased, if necessary. The dosage required to trigger accelerated involution was continued until the lesion had completely involuted or until the child was 12 months of age, when the medication was withdrawn over 2 weeks. The patients were followed-up for 6.5-18.0 (mean, 11.8 months) months.

\section{Results}

A total of 15 consecutive patients ( 5 male and 10 female) aged 3 weeks to 8.5 months (mean, 11 weeks) with problematic proliferating haemangioma were treated with propranolol. Six infants were born 2.5-6 (mean 4.2 weeks) weeks, prematurely. The haemangioma affected the nose $(n=2)$, nose and upper lip $(n=2)$, nose and cheek $(n=1)$, medial canthus $(n=1)$, medial canthus and cheek $(n=1)$, ear and parotid area $(n=1)$, breast $(n=1)$, scapular area $(n=1)$, peri-anal area $(n=2)$, shoulder $(n=1)$ and hand $(n=1)$. Four of these patients had lesions elsewhere in the body. Another patient had multiple cutaneous and hepatic haemangioma causing severe liver dysfunction with obstructive jaundice. The haemangioma threatened life $(n=1)$ or vision $(n=2)$ or caused nasal obstruction $(n=3)$ and/or ulceration $(n=6)$ and/or bleeding $(n=2)$ and/or significant tissue distortion $(n=12)$.

Propranolol at $1.5 \mathrm{mg} \mathrm{kg}^{-1} \mathrm{day}^{-1}$ in 13 patients and at $2 \mathrm{mg} \mathrm{kg}^{-1}$ day $^{-1}$ in two patients was required to achieve accelerated involution of the haemangioma. The markedly deranged liver function in the patient with multiple hepatic and cutaneous haemangioma normalised with corresponding dramatic shrinkage of the liver lesions, within 3 months of treatment. A similar dramatic response was observed in all other cases, except in one premature infant, who had borderline asymptomatic diastolic hypotension initially requiring slow increments of Propranolol to $2 \mathrm{mg} \mathrm{kg}^{-1} \mathrm{day}^{-1}$ over 2 months, which resulted in accelerated involution of the haemangioma. 
Rebound growth occurred in the first patient treated in this series when the dose was withdrawn at 7.5 months of age requiring reinstitution of propranolol at $1.5 \mathrm{mg} \mathrm{kg}^{-1} \mathrm{day}^{-1}$, which was continued until the child was 12 months old. No rebound growth was observed in the remaining patients, who were maintained on the treatment until 12 months of age or earlier when the lesion had completely involuted. The treatment was then withdrawn over a 2-week period. No complications were observed.

Figures 1-5 show some of the cases treated with propranolol.

\section{Case 1}

A 9-week-old boy presented with a proliferating haemangioma involving the upper lip and nose (Figure 1A and B) causing ulceration and bleeding with feeding and breathing difficulties. He was treated with high-dose systemic steroid for 1 month without effect and was admitted to the local hospital because of poor weight gain and requiring nasogastric feeding. The patient was referred to our Vascular Anomalies Centre and was commenced on propranolol at $0.25 \mathrm{mg} \mathrm{kg}^{-1}$ twice daily for $24 \mathrm{~h}$, increasing to $1.5 \mathrm{mg} \mathrm{kg}^{-1}$ twice daily. There was visible shrinkage of the haemangioma within $24 \mathrm{~h}$ (Fig. 1C), with ongoing accelerated involution over the next 6.5 months (Fig. 1D). The treatment was tapered over 2 weeks when he was 7.5 months old. Rebound growth occurred requiring reinstitution of propranolol at $1.5 \mathrm{mg}^{-1} \mathrm{~kg}^{-1}$, which was discontinued at 12 months of age.
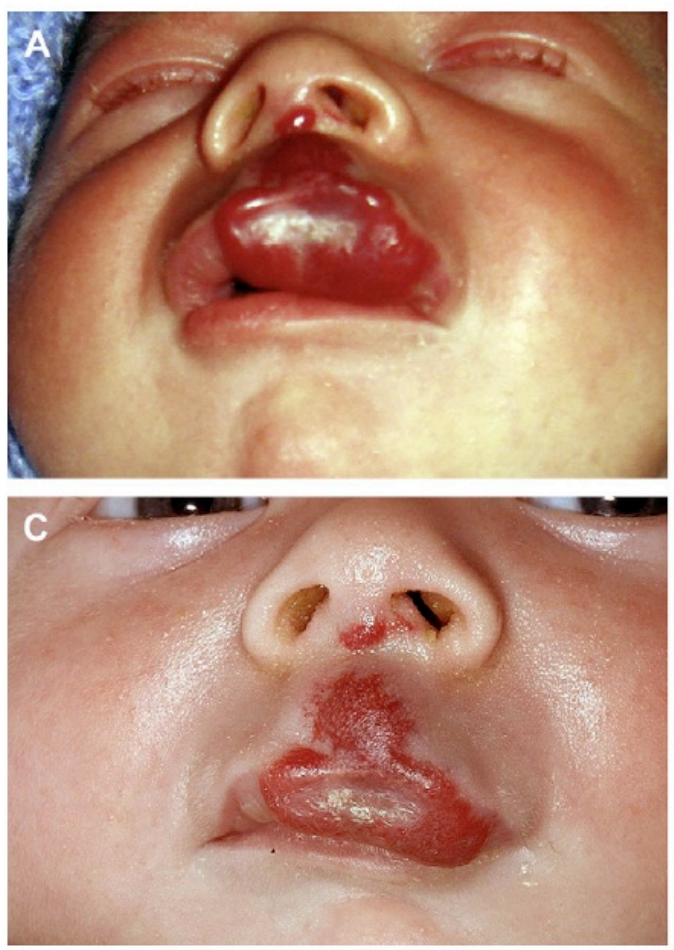

\section{Case 2}

A 7-week-old girl presented with a rapidly growing haemangioma occupying almost the entire nose with nasal obstruction (Figure 2A) and a separate lesion on the abdomen and mastoid skin. The patient was commenced on propranolol and the dose was increased to $1.5 \mathrm{mg} \mathrm{kg}^{-1} \mathrm{day}^{-1}$, resulting in accelerated involution. A dramatic effect was noted with marked reduction of the size at 3 weeks (Figure 2B), and continued improvement at 3.5 months (Figure $2 \mathrm{C}$ ) and virtual complete involution at 8 months (Figure 2D) after treatment. The dose was maintained until 1 -year of age.

\section{Case 3}

A 3-week-old girl, born 4 weeks prematurely, presented with a proliferating haemangioma in the parotid area and ear with ulceration causing pain and distortion of the ear (Figure 3A). Accelerated involution was noted within $48 \mathrm{~h}$ (Figure $3 \mathrm{~B}$ ) as was ongoing improvement over the next 8 months' (Figure 3 C and $D$ ) treatment with propranolol at $1.5 \mathrm{mg} \mathrm{kg}^{-1}$ day $^{-1}$.

\section{Case 4}

An 8.5-month old boy, born 3.5 weeks prematurely, presented with a haemangioma distorting the nose and causing nasal obstruction (Figure 4A). Accelerated involution was observed following propranolol treatment at
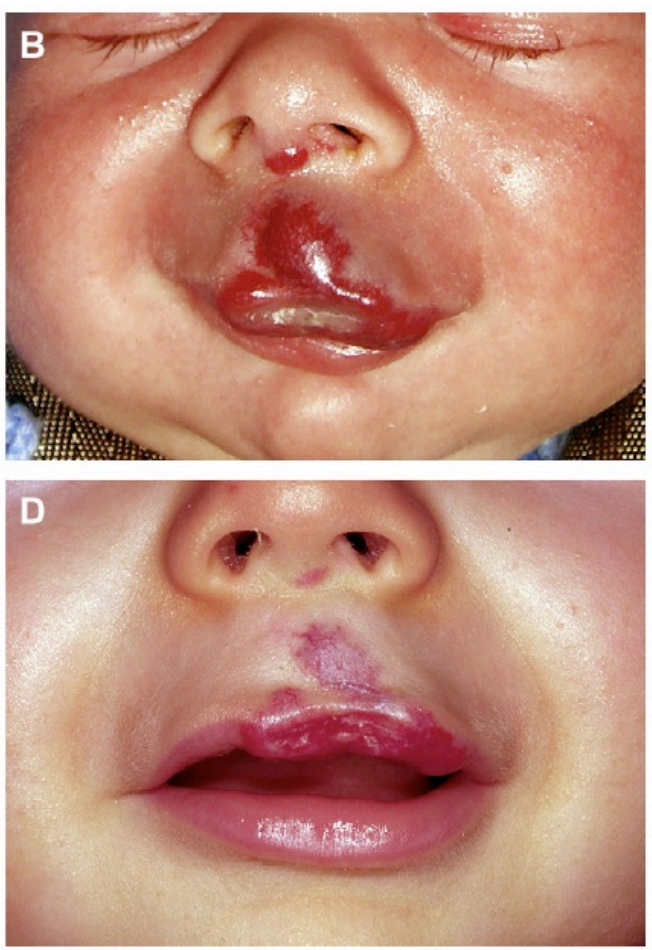

Figure 1 A 9-week-old boy with a steroid-resistant proliferating haemangioma involving the upper lip and nose (A). $24 \mathrm{~h}$ (B), and 6.5 months $(\mathrm{C})$ after propranolol treatment. 

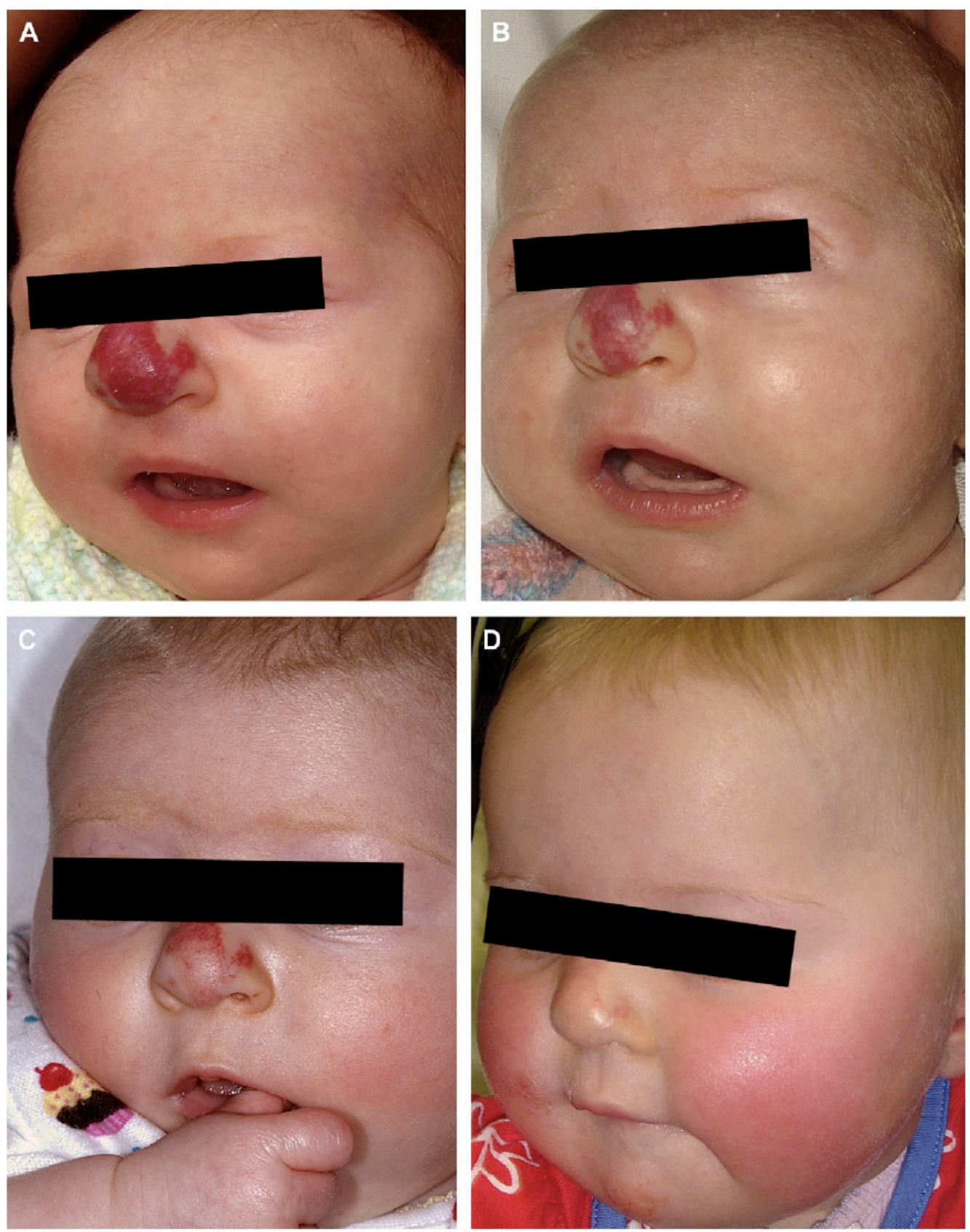

Figure 2 A 7-week-old girl with a proliferating haemangioma involving the nose (A) and a separate lesion on the abdomen and mastoid skin. Dramatic response to propranolol treatment at 2 weeks (B) and continued improvement at 6 weeks (C) and virtual complete involution 8 months (D) after treatment.

$1.5 \mathrm{mg} \mathrm{kg}^{-1}$ day $^{-1}$ (Figure $4 \mathrm{~B}$ and $\mathrm{C}$ ). The treatment was continued until he was aged 15 months.

\section{Case 5}

A 4-month- old girl presented with a large haemangioma on the right breast causing significant distortion (Figure 5A). Treatment with propranolol at $1.5 \mathrm{mg} \mathrm{kg}^{-1} \mathrm{day}^{-1}$ resulted in accelerated involution and ongoing improvement over the next 5 months (Figure 5 B-D).

\section{Discussion}

Since steroids were serendipitously discovered to induce accelerated involution of proliferating infantile haemangioma, ${ }^{10,11,30}$ they have remained the first-line 

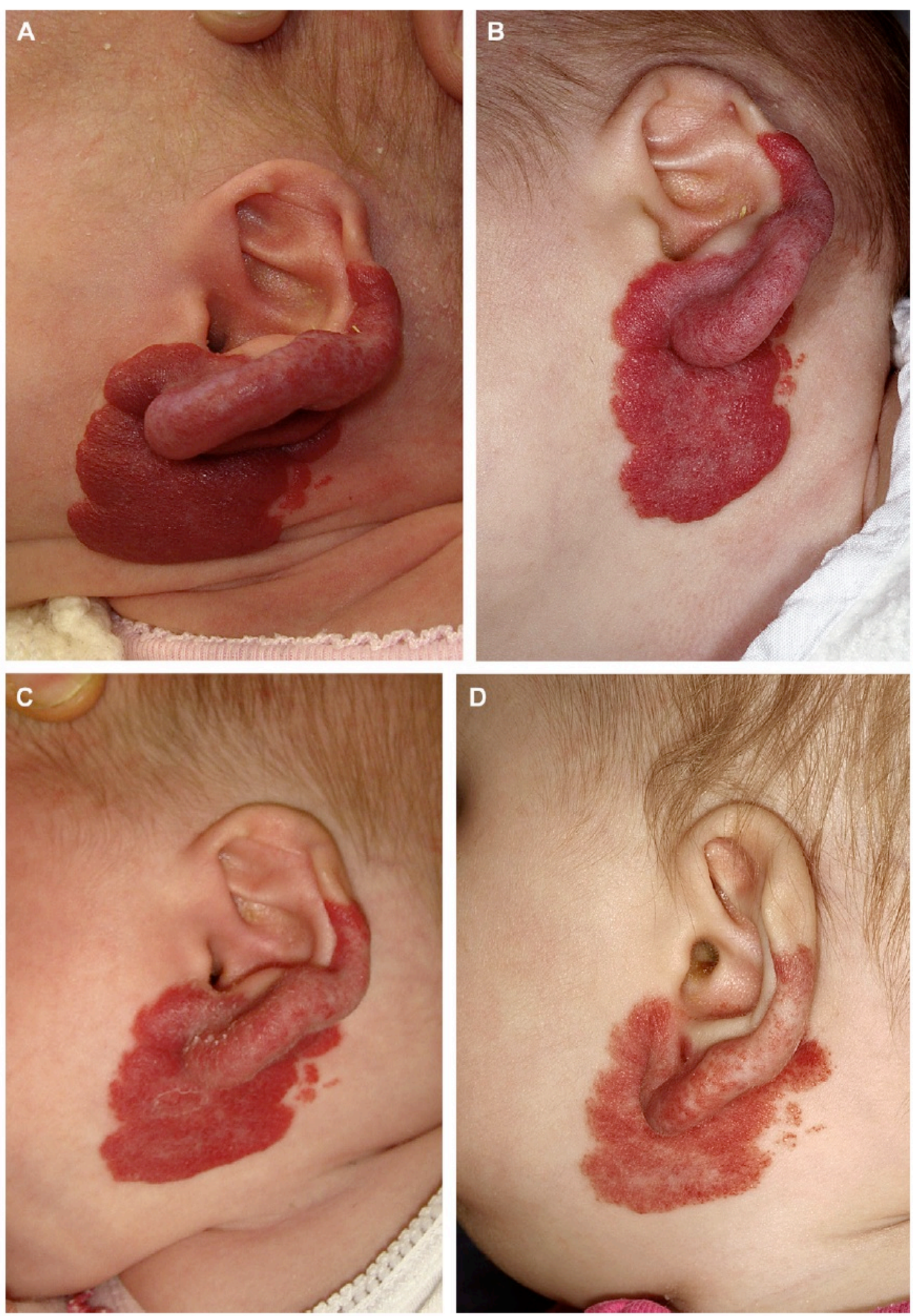

Figure 3 A 3-week-old girl with a proliferating haemangioma in the parotid area and ear with ulceration causing pain and distortion of the ear (A). Accelerated involution was noted 2 days (B), 7 weeks (C), and 6 months (D) after treatment with propranolol. (2010), doi:10.1016/j.bjps.2010.06.010 

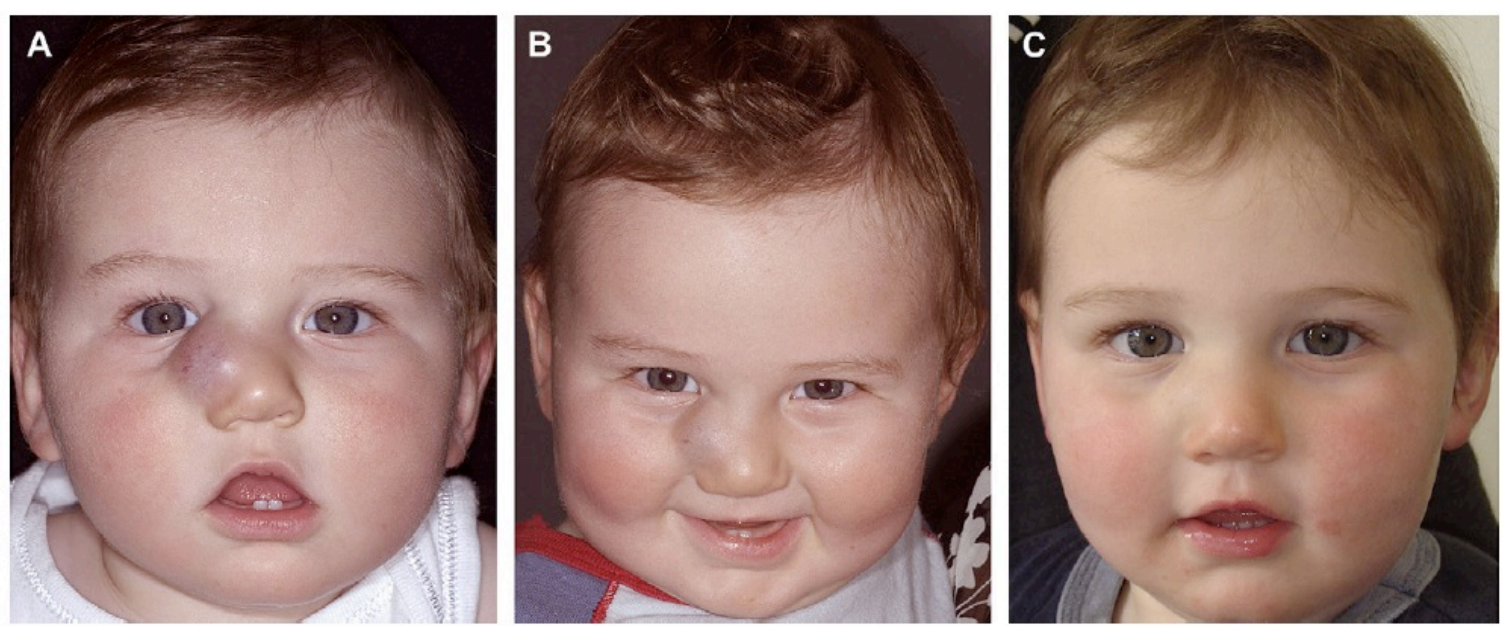

Figure 4 An 8.5-month old boy with a haemangioma distorting the nose and causing nasal obstruction (A). Accelerated involution was observed 3 weeks (B) and 6 months (C) after propranolol treatment which was continued until he was aged 15 months.
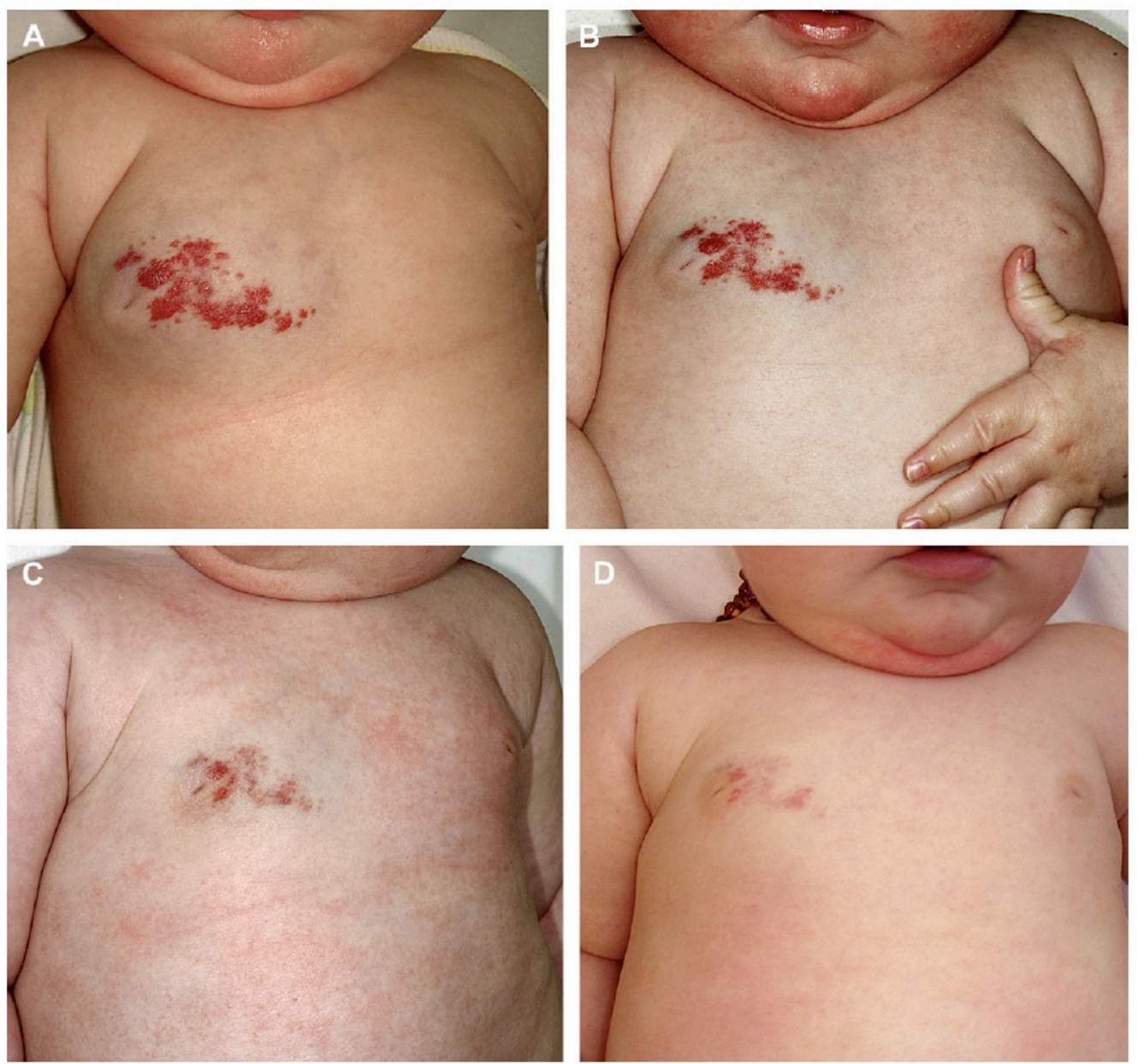

Figure 5 A 4-month old girl with presented with a large haemangioma on the right breast causing significant distortion (A). Accelerated involution at 1 week (B), 1 month (C), and 3 months (D) after treatment with propranolol. 
treatment for problematic proliferating lesions. ${ }^{2,5,7,9,12}$ Vincristine has emerged as an alternative to interferon as a second-line treatment for steroid-resistant cases because of significant adverse side effects. ${ }^{17,18}$

In 2008, Léatué-Labrèze et al. serendipitously discovered the dramatic effect of Propanolol in inducing accelerated involution of infantile haemangioma. ${ }^{22}$ Similar results of treatment with propranolol at an empirical dosage of $2-3 \mathrm{mg} \mathrm{kg}^{-1} \mathrm{day}^{-1}$ have been reported by others. ${ }^{23-26}$ We confirm, in this report, the efficacy of propranolol and show that the minimal dosage required to achieve accelerated involution is a sub-cardiovascular dose of $1.5-2.0 \mathrm{mg} \mathrm{kg}^{-1}$ day $^{-1}$ and that the treatment should be continued until the lesion is fully involuted or the child is 1 -year of age.

Historically, $\beta$-blockers have been used in infants for cardiac indications such as tetralogy of Fallot $^{27}$ and hypertrophic cardiomyopathy. ${ }^{28}$ Although the experience with $\beta$-blockers in the paediatric population is extensive, its safety profile is not well established for this new indication. The side effects of $\beta$-blockers are well known and include transient bradycardia, hypotension and bronchospasm in patients with underlying reactive airways. Reduction in lipolysis, glycogenolysis and gluconeogenesis by $\beta$-blockers predispose the patients to hypoglycaemia, and is particularly relevant in very young infants with potential adverse neurological sequelae. ${ }^{29}$

We find that low-dose propranolol administered in divided doses with gradual increase is safe. However, the potential side effects of propranolol necessitate particular care when initiating treatment and during dose escalation, especially in very young infants. Larger-scale studies confirming the safety and efficacy of propranolol may broaden the indications of treatment of proliferating infantile haemangioma.

The processes leading to accelerated involution of proliferating infantile haemangioma induced by propranolol are unknown. Hypotheses regarding the effect of $\beta$-blockers on infantile haemangioma include vasoconstriction, which is immediately visible as a change in colour and palpable softening of the haemangioma; ${ }^{23}$ decreased expression of vascular endothelial growth factor and basic fibroblast growth factor genes through the down-regulation of the RAF-mitogen-activated protein kinase pathway; ${ }^{31}$ and apoptosis of capillary endothelial cells. ${ }^{32,33}$

Renin levels in the newborns are 14 times that of the adults, and these levels are halved by 1 -year of age with gradual tapering to the adult levels by age $10 .^{34}$ Black infants have lower renin levels than white infants ${ }^{35}$ and female infants have higher renin levels than age-matched males. ${ }^{36}$ Premature birth is also associated with higher circulating renin activity. ${ }^{37}$

We have shown a crucial role for the renin-angiotensin system (RAS) in infantile haemangioma, ${ }^{38}$ which is supported by the clinical observation of a higher incidence of this tumour in Caucasian, female and premature infants. ${ }^{1,39}$

We have also recently shown that infantile haemangioma is a developmental anomaly of primitive mesoderm-derived haemogenic endothelium ${ }^{40}$ with a neural crest phenotype. ${ }^{41}$ We further show that the endothelial progenitor cells within proliferating haemangioma are governed by the RAS. ${ }^{38}$ We propose that RAS can account for both the action of $\beta$-blockers and the process of spontaneous involution of infantile haemangioma and we speculate upon the possibility of using inhibitors of angiotensin-converting enzyme and that of angiotensin II receptor 2 as alternative therapeutic regimes. ${ }^{38}$

\section{Conflict of interest}

None.

\section{References}

1. Takahashi K, Mulliken JB, Kozakewich HP, et al. Cellular markers that distinguish the phases of hemangioma during infancy and childhood. J Clin Invest 1994;93:2357-64.

2. Frieden IJ, Eichenfield LF, Esterly NB, et al. J Am Acad Dermatol 1997;37:631-7.

3. Tan ST, Velickovic M, Ruger BM, et al. Cellular and extracellular markers of hemangioma. Plast Reconstr Surg 2000;106: 529-37.

4. Mulliken JB, Glowacki J. Hemangiomas and vascular malformations in infants and children: a classification based on endothelial characteristics. Plast Reconstr Surg 1982;69: 412-9.

5. Drolet BA, Esterly NB, Frieden IJ. Hemangiomas in children. N Engl J Med 1999;341:173-81.

6. Chang LC, Haggstrom AN, Drolet BA, et al. Growth characteristics of infantile hemangiomas: implications for management. Pediatrics 2008;122:360-7.

7. Enjolras O, Gelbert F. Superficial hemangiomas: associations and management. Pediatr Dermatol 1997;14:173-9.

8. Tan B, Leadbitter P, Auburn N, Tan ST. Steroid therapy for problematic proliferating haemangioma. 2010 (Submitted).

9. Gangopadhyay A, Sinha C, Gopal S, et al. Role of steroid in childhood haemangioma: a 10 years review. Int J Surg 1997;82: 49-51.

10. Zarem HA, Edgerton MT. Induced resolution of cavernous hemangiomas following prednisolone therapy. Plast Reconstr Surg 1967;39:76-83.

11. Fost NC, Esterly NB. Successful treatment of juvenile hemangiomas with prednisone. J Pediatrics 1968;72:351-7.

12. Bennett ML, Fleischer Jr AB, Chamlin SL, et al. Oral cortico steroid use is effective for cutaneous hemangiomas: an evidence-based evaluation. Arch Dermatol 2001;137:1208-13.

13. Boon LM, MacDonald DM, Mulliken JBMD. Complications of systemic corticosteroid therapy for problematic hemangioma. Plast Reconstr Surg 1999;104:1616-23.

14. Beck DO, Gosain AK. The presentation and management of hemangiomas. Plast Reconstr Surg 2009;123:181e-91e.

15. Greinwald Jr JH, Burke DK, Bonthius DJ, et al. An update on the treatment of hemangiomas in children with interferon alfa2a. Arch Otolaryngol Head Neck Surg 1999;125:21-7.

16. Barlow CF, Priebe CJ, Mulliken JB, et al. Spastic diplegia as a complication of Interferon alfa-2a treatment of hemangiomas of infancy. J Pediatrics 1998;132:527-30.

17. Fawcett SL, Grant I, Hall PN, et al. Vincristine as a treatment for a large haemangioma threatening vital functions. BJPS 2004;57:168-71.

18. Wharton $\mathrm{S}$, Soueid $\mathrm{A}$, Nishikawa $\mathrm{H}$, et al. Endangering cutaneous infantile hemangioma treated with vincristine: a case report. Eur J Plast Surg 2009;32:157-61.

19. Batniji RK, Buckingham ED, Williams EF. An aesthetic approach to facial hemangiomas. Arch Facial Plast Surg 2005;7:301-6.

20. Williams EF, Stanislaw P, Dupree $M$, et al. Hemangiomas in infants and children an algorithm for intervention. Arch Facial Plast Surg 2000;2:103-11. 
21. Thomas RF, Hornung RL, Manning SC, et al. Hemangiomas of infancy. Arch Facial Plast Surg 2005;7:312-5.

22. Leaute-Labreze C, Dumas de la Roque E, Hubiche T, et al. Propranolol for severe hemangiomas of infancy. N Engl J Med 2008;358:2649-51.

23. Sans V, de la Roque ED, Berge J, et al. Propranolol for severe infantile hemangiomas: follow-up report. Pediatrics 2009;124: e423-431.

24. Buckmiller L, Dyamenahalli U, Richter GT. Propranolol for airway hemangiomas: case report of novel treatment. Laryngoscope 2009; 73:2051-4.

25. Denoyelle F, Leboulanger N, Enjolras O, et al. Role of propranolol in the therapeutic strategy of infantile laryngotracheal hemangioma. Int J Pediatr Otorhinolaryngol 2009;73: $1168-72$.

26. Manunza F, Syed S, Laguda B, et al. Propranolol for complicated infantile haemangiomas: a case series of 30 infants. $\mathrm{Br} J$ Dermatol 162:466-468.

27. Honey M, Chamberlain DA, Howard J. The effect of betasympathetic blockade on arterial oxygen saturation in Fallot's Tetralogy. Circulation 1964;30:501-10.

28. Cherian G, Brockington I, Shah P, et al. Beta-adrenergic blockade in hypertrophic obstructive cardiomyopathy. BMJ 1966;1:895-8.

29. Siegfried EC, Keenan WJ, Al-Jureidini S, et al. More on propranolol for hemangiomas of infancy. N Engl J Med 2008; 359:2846-7.

30. Edgerton MT. The treatment of hemangiomas: with special reference to the role of steroid therapy. Ann Surg 1976;183:517-30.

31. D'Angelo G, Lee H, Weiner RI. cAMP-dependent protein kinase inhibits the mitogenic action of vascular endothelial growth factor and fibroblast growth factor in capillary endothelial cells by blocking Raf activation. J Cell Biochem 1997;67: 353-66.

32. Smith SKS, Smith DM. Beta blockade induces apoptosis in cultured capillary endothelial cells. Vitro Cell Dev Biol. 2002; 38:298-304.

33. laccarino $G$, Ciccarelli $M$, Sorriento $D$, et al. Ischemic neoangiogenesis enhanced by $\{$ beta\}2-adrenergic receptor overexpression: a novel role for the endothelial adrenergic system. Circ Res. 2005;97:1182-9.

34. Dillon MJ, Ryness JM. Plasma renin activity and aldosterone concentration in children. BMJ 1975;4:316-9.

35. Youmbissi TJ, Tedong F, Fairbank ST, et al. Plasma renin activity studies in a group of African neonates and children. $J$ Trop Pediatr 1990;36:128-30.

36. Pipkin FB, Smales OR, O'Callaghan MJ. Renin and angiotensin levels in children. Arch Dis Child 1981;56:298-302.

37. Stephenson TJ, Pipkin FB, Elias-Jones AC. Factors influencing plasma renin and renin substrate in premature infants. Arch Dis Child 1991;66:1150-4.

38. Itinteang T, Brasch HD, Tan ST, Day DJ. Expression of components of the renin-angiotensin system in haemangioma may account for Propranolol-induced accelerated involution. JPRAS (in press).

39. North PE, Waner M, Mizeracki A, et al. A unique microvascular phenotype shared by juvenile hemangiomas and human placenta. Arch Dermatol 2001;137:559-70.

40. Itinteang T, Tan ST, Brasch H, Day DJ. Haemogenic endothelium - the origin of infantile haemangioma? J Clin Pathol. 2010. (Submitted for publication.)

41. Itinteang T, Brasch HD, Tan ST, Day DJ. Primitive mesodermal cells with a neural crest stem cell phenotype predominate proliferating infantile haemangioma. J Clin Pathol. (in press). 
11.4. Manuscript: Low-Dose Propranolol For Multiple Infantile Hepatic And Cutaneous Hemangiomas With Deranged Liver Function 


\section{Low-Dose Propranolol for Multiple Infantile Hepatic and Cutaneous Hemangiomas With Deranged Liver Function}

\author{
AUTHORS: Swee Thong Tan, MBBS, FRACS, PhD, a,b,c Tinte \\ Itinteang, MBBS, a,b and Philip Leadbitter, MBBS, FRACPa,d \\ ${ }^{a}$ Centre for the Study and Treatment of Vascular Birthmarks, \\ Wellington Regional Plastic, Maxillofacial and Burns Unit, Hutt \\ Hospital, Wellington, New Zealand; ${ }^{b}$ Gillies McIndoe Research \\ Institute, Lower Hutt, New Zealand; $\bullet \bullet \bullet \bullet \bullet \bullet$, University of \\ Otago, Dunedin, New Zealand; and d Department of Paediatrics, \\ Hutt Hospital, Wellington, New Zealand

\section{KEY WORDS} \\ infantile, hemangioma, propranolol, $\beta$-blocker, renin, \\ angiotensin, treatment, involution \\ ABBREVIATION \\ $\mathrm{IH}$-infantile hemangioma
}

This article was presented in part at the Royal Australasian College of Surgeons' annual scientific congress, May 11-16, 2009, Brisbane, Australia; the Australian and New Zealand Vascular Anomalies Interest Group meeting, 0ctober 22, 2009, Melbourne, Australia; the New Zealand Association of Plastic Surgeons' annual scientific meeting, November 21, 2009, Auckland, New Zealand; and an International Society for the Study of Vascular Anomalies meeting. April 21-24, 2010 , Brussels, Belgium.

www.pediatrics.org/cogi/doi/10.1542/peds.2010-1703 doi:10.1542/peds.2010-1703

Accepted for publication Nov 18, 2010

Address correspondence to Swee Thong Tan, MBBS, FRACS, PhD, Wellington Regional Plastic, Maxillofacial and Burns Unit, Hutt Hospital, High Street, Private Bag 31-907, Lower Hutt, New Zealand. E-mail: swee.tan@huttvalleydhb.org.nz

PEDIATRICS (ISSN Numbers: Print, 0031-4005; Online, 1098-4275). Copyright @ 2011 by the American Academy of Pediatrics FINANCIAL DISCLOSURE: The authors have indicated they have no financial relationships relevant to this article to disclose.

\section{abstract}

We report here the case of an infant with multiple hepatic and cutaneous infantile hemangiomas ( $\mathrm{IHs}$ ) associated with deranged liver function who was treated successfully with low-dose propranolol. We also discuss our recent data that show that $\mathrm{IH}$ is a developmental anomaly of hemogenic endothelium derived from primitive mesoderm with a neural crest-cell phenotype. We previously presented evidence that this hemogenic endothelium is governed by the renin-angiotensin system, which we propose can account for both the action of propranolol and the process of spontaneous involution of $\mathrm{IH}$. We further speculate on the possibility of using inhibitors of angiotensinconverting enzyme and that of angiotensin II receptor 2 as potential alternative therapies. Pediatrics 2011;127:e000 
Infantile hemangioma $(\mathrm{IH})$ is the most common tumor of infancy with a preponderance in white, female, and premature infants. ${ }^{1}$ It is characterized by rapid proliferation during infancy followed by slow involution over the next 1 to 5 years with continued improvement up to 10 years when the cellular elements are gradually replaced by fibrofatty deposition. ${ }^{2,3}$

IH usually presents as a solitary lesion that affects the skin and/or subcutaneous tissue. ${ }^{14} \mathrm{~A}$ subgroup of $\mathrm{IHs}$ are distributed in a regional fashion, ${ }^{5}$ and some are associated with midline structural anomalies and constitute PHACES syndrome (posterior fossa malformations, hemangioma, arterial anomalies, cardiac defects, eye abnormalities, and supraumbilical raphe) ${ }^{6}$ Multiple cutaneous lesions are not uncommon, and the incidence of hepatic involvement is related to the number of cutaneous lesions. ${ }^{7}$

Approximately $10 \%$ of IHs require intervention during infancy, because the lesion poses a threat to life or function or causes tissue distortion or destruction. 8,9 The mainstay treatment during the proliferative phase has been pharmacologic therapy. High-dose steroid is the first-line treatment, ${ }^{1,10-12}$ and its adverse effects are well recognized. ${ }^{12}$ The second-line treatment for problematic proliferating $\mathrm{IH}$ has been interferon. ${ }^{13}$ Because interferon is associated with significant adverse effects, ${ }^{14,15}$ vincristine has emerged as an alternative for steroid-resistant cases. ${ }^{16,17}$

Severe cases of hepatic $\mathrm{IH}$ are often associated with a high mortality rate despite aggressive and combined treatments. ${ }^{18}$ Subcategorization of this condition and its treatment algorithm were proposed more recently ${ }^{19}$ to provide a rational approach and indications for active treatment.

Propranolol was discovered serendipitously in 2008 to induce accelerated in-
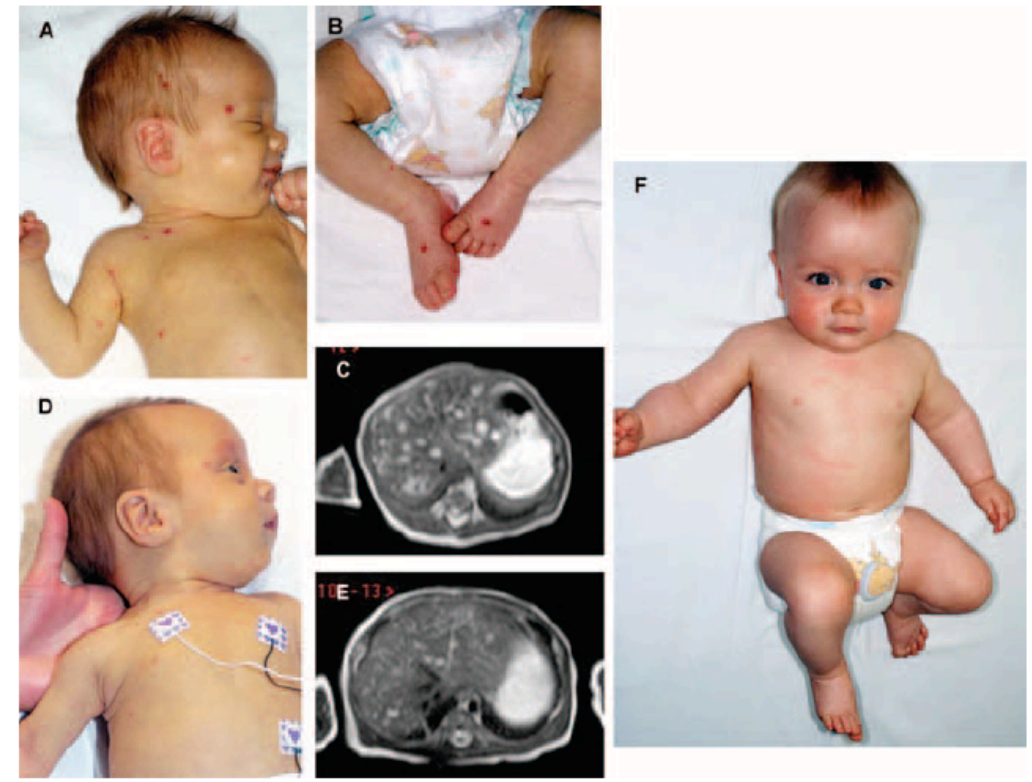

FIGURE 1

A and B, A 3-week-old boy with multiple cutaneous and hepatic IHs associated with deranged liver function. C, An MRI scan shows multiple hepatic lesions. Also shown are accelerated involution of the cutaneous lesions (D) and marked reduction in size of the hepatic lesions (E) after 4 weeks of treatment. F, The patient 8 months after treatment.

volution of a large problematic facial proliferating $\mathrm{IH}$; it was used to treat steroid-induced hypertrophic cardiomyopathy. ${ }^{13} \mathrm{~A}$ limited number of case reports and case series have confirmed this biological effect of propranolol on proliferating $1 \mathrm{H},{ }^{13-18}$ and we have shown that a subcardiovascular dosage with gradual increase seems to be effective and safe. ${ }^{20}$

We present here a case in which treatment with low-dose propranolol for an infant with multiple hepatic and cutaneous IHs associated with deranged liver function was successful.

\section{CASE REPORT}

A 3-week-old boy was referred to the Centre for the Study and Treatment of Vascular Birthmarks with multiple cutaneous (Fig $1 \mathrm{~A}$ and $\mathrm{B}$ ) and hepatic $\mathrm{HHS}$ associated with increasing liver dysfunction (total serum bilirubin: $269 \mathrm{U} / \mathrm{L}$ [69\% conjugated]; elevation liver enzyme levels) (Fig 2). He was born at 39.5 weeks by cesarean delivery and developed mild jaundice at day 7 with $60 \mathrm{~g}$ of weight loss having regained his birth weight of $3.1 \mathrm{~kg}$. Examination showed that the liver was enlarged at 2 $\mathrm{cm}$ below the costal margin. An ultrasound scan of the abdomen revealed an enlarged liver with multiple hypoechoic areas with increased blood flow. There was no clinical or echocardiographic evidence of cardiac failure, and electrocardiogram results were normal. A full blood count revealed a hemoglobin level of $101 \mathrm{~g} / \mathrm{L}$ and a white cell count of 5.7 (neutrophils: $1.4 \times$ $10^{9} / \mathrm{L}$ ), and the results of clotting studies were normal. Results of thyroidfunction tests were normal on several occasions. An MRI confirmed multiple high-flow hepatic IHs causing enlargement of the liver (Fig 1C). The patient was commenced on propranolol at $0.25 \mathrm{mg} / \mathrm{kg}$ orally twice daily as an inpatient; his heart rate, blood pressure, and preprandial blood glucose level were measured every 4 hours. There were no cardiovascular or metabolic 


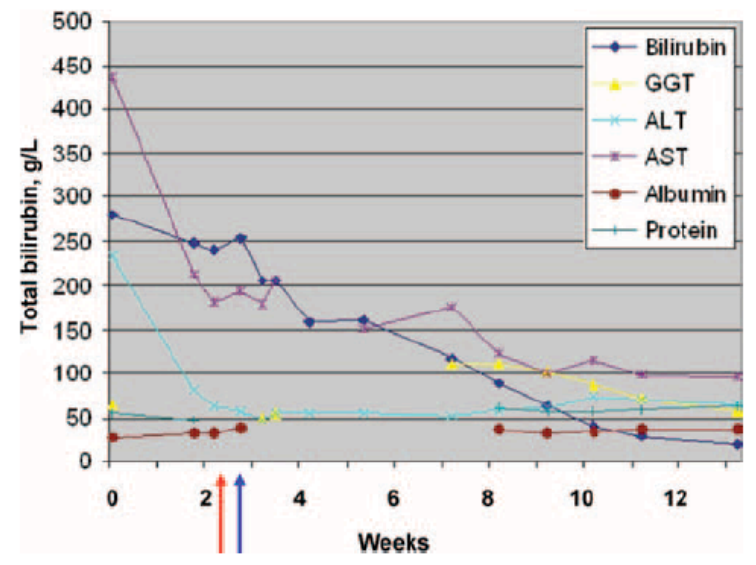

FIGURE 2

Rapid improvement of liver-function test results after commencement of propranolol. Deterioration in liver function occurred when propranolol was stopped (red arrow) for 4 days during an intercurrent bronchiolitis, 17 days after commencement of propranolol. Liver function improved rapidly when the treatment was recommenced (blue arrow) and virtually normalized after 3 months of treatment. GGT indicates gamma-glutamyl transpeptidase; ALT, alanine aminotransferase; AST, aspartate aminotransferase.

adverse effects observed, and there was a visible involution of the cutaneous IHs within 24 hours. The dosage was increased by $0.5 \mathrm{mg} / \mathrm{kg}$ daily every 24 hours to $1.5 \mathrm{mg} / \mathrm{kg}$ per day. The patient was maintained on this dosage and then followed up in the outpatient clinic at 1 week, every 2 weeks for 3 months, and then every 2 months afterward.

Liver-function test results improved within 3 days of commencing on propranolol and virtually normalized after 3 months of treatment (Fig 2). The accelerated involution of the cutaneous lesions (Fig 1D) mirrored the marked reduction in size of the hepatic lesions on repeat MRI scan after 4 weeks of treatment (Fig 1E). Seventeen days after commencing on propranolol, the patient developed apneic episodes and brief runs of sinus bradycardias during an intercurrent bronchiolitis that required hospital admission. Deterioration in his liver-function test results was noted when propranolol was stopped for 4 days but improved rapidly when the treatment was recommenced (Fig 2).
A repeat MRI showed that the hepatic sions, had virtually disappeared after 4 months of propranolol treatment. There was ongoing improvement, and the dosage was maintained until the child was 12 months of age, at which time the medication was withdrawn over 4 weeks. We did not observe any adverse effect of propranolol including bradycardia, hypotension, hypoglycemia, or cold periphery during the treatment period. Rebound growth was not observed during the 16-month follow-up (Fig 1F).

\section{DISCUSSION}

Since steroids were serendipitously discovered to induce accelerated invoIution of proliferating $\mathrm{IHs}$ in the $1960 s,{ }^{19,21,22}$ they have remained the first-line treatment for problematic proliferating lesions. ${ }^{1,8,10,12,23}$ Vincristine $e^{24,25}$ has emerged as an alternative second-line therapy for steroidresistant cases because of significant adverse effects of interferon, including dysplastic diplegia, which occurs in $25 \%$ of cases. ${ }^{24,25}$ lesions, along with the cutaneous le-
Not all patients with multiple cutaneous and/or hepatic IHs require active treatment. Severe cases of hepatic IH are associated with a high mortality rate despite aggressive and, often, combined treatments. ${ }^{18}$ Subcategorization and a treatment algorithm for this condition were developed more recently ${ }^{19}$ to provide a rational approach and indications for active treatment for this condition. The importance of excluding severe hypothyroidism caused by induction of type 3-iodothyronine deiodinase in hepatic $\mathrm{IH}$, and to institute thyroid hormone replacement therapy, is well recognized. ${ }^{20}$

In 2008, propranolol was serendipitously observed to induce accelerated involution of $\mathrm{IH}^{13}$ Similar results of treatment with propranolol at an empirical dosage of 2 to 3 $\mathrm{mg} / \mathrm{kg}$ per day have been reported by others. ${ }^{14-17}$

Historically, $\beta$-blockers have been used in infants for cardiac indications such as tetralogy of Fallot ${ }^{26}$ and hypertrophic cardiomyopathy. ${ }^{27}$ Although the experience with $\beta$-blockers in the pediatric population is extensive, its safety profile has not been well established for this new indication. The adverse effects of $\beta$-blockers are well known and include transient bradycardia, hypotension, and bronchospasm in patients with underlying reactive airways. Reduction in lipolysis, glycogenolysis, and gluconeogenesis by $\beta$-blockers predispose patients to hypoglycemia, which is particularly relevant in very young infants with potential adverse neurologic sequelae. ${ }^{28} \mathrm{~A}$ divided dosing and nonprecipitous escalation to the dose regimen may add a margin of safety and provide an opportunity to use a minimal dosage to achieve the desired effect of propranolol..$^{29}$ We recently showed that a subcardiovascular dose of 1.5 to $2.0 \mathrm{mg} / \mathrm{kg}$ per day administered in divided doses 
with gradual increase is effective and safe, and we have recommended that treatment be continued until the lesion is fully involuted or the child is 1 year of age. ${ }^{18}$

Multicenter clinical trials are currently underway to confirm the safety and efficacy of propranolol in the treatment of $I H$, which may broaden its indications for the treatment of proliferating $\mathrm{IH}^{2}{ }^{28}$

We have proposed a crucial role for the renin-angiotensin system in the biol-

\section{REFERENCES}

1. Frieden IJ, Eichenfield LF, Esterly NB, Geronemus R, Mallory SB. The Guidelines of care for hemangiomas of infancy. American Academy of Dermatology, Guidelines/ Outcomes Committee. J Am Acad Dermatol. 1997;37(4):631-637

2. Tan ST, Velickovic M, Ruger BM, Davis PF. Cellular and extracellular markers of hemangioma. Plast Reconstr Surg. 2000;106 (3): 529-537

3. Mulliken JB, Glowacki J. Hemangiomas and vascular malformations in infants and children: a classification based on endothelial characteristics. Plast Reconstr Surg. 1982;69(3):412-419

4. Takahashi K, Mulliken JB, Kozakewich HP. Rogers RA, Folkman J, Ezekowitz RAB. Cellular markers that distinguish the phases of hemangioma during infancy and childhood. J Clin Invest. 1994;93(6):2357-2364

5. Waner M, North PE, Scherer KA, Frieden IJ, Waner A, Mihm MC Jr. The nonrandom distribution of facial hemangiomas. Arch Dermatol. 2003;139(7):869-875

6. Metry DW, Hawrot A, Altman C, Frieden IJ. Association of solitary, segmental hemangiomas of the skin with visceral hemangiomatosis. Arch Dermatol. 2004;140(5): 591-596

7. Horii KA, Drolet BA, Baselga E, et al; Hemangioma Investigator Group. Risk of hepatic hemangiomas in infants with large hemangiomas. Arch Dermatol. 2010;146(2): 201-203

8. Drolet BA, Esterly NB, Frieden IJ. Hemangiomas in children. N Engl J Med. 1999;341(3) 173-181

9. Chang LC, Haggstrom AN, Drolet BA, et al; Hemangioma Investigator Group. Growth characteristics of infantile hemangiomas: ogy of $\mathrm{IH},{ }^{30}$ which is supported by the clinical observation of a higher incidence of this tumor in white, female, and premature infants.,31 This observation implies the need to continue treatment until the lesion is fully involuted or at least until 1 year of age. ${ }^{18}$

We have also shown recently that $\mathrm{IH}$ is a developmental anomaly of hemogenic endothelium derived from primitive mesoderm ${ }^{32}$ with a neural crestcell phenotype. ${ }^{33}$ We have further presented evidence that this hemogenic endothelium is governed by the

implications for management. Pediatrics. 2008;122(2):360-367

10. Enjolras 0, Gelbert F. Superficial hemangiomas: associations and management. Pediatr Dermatol. 1997;14(3):173-179

11. Tan B, Leadbitter P. Auburn N, Tan ST. Steroid therapy for problematic proliferating haemangioma. N Z Med J. 2010; In press

12. Gangopadhyay A, Sinha C, Gopal S, Gupta D, Sahoo S, Ahmad M. Role of steroid in childhood haemangioma: a 10 years review. Int Surg. 1997;82(1):49-51

13. Léauté-Labrèze C, Dumas de la Roque E, Hubiche T, Boralevi F, Thambo JB, Taïeb A. Propranolol for severe hemangiomas of infancy. N Engl J Med. 2008;358(24): 2649-2651

14. Sans V, de la Roque ED, Berge J, et al. Propranolol for severe infantile hemangiomas: follow-up report. Pediatrics. 2009;124(3). Available at: www.pediatrics.org/cgi/ content/full/124/3/e423

15. Denoyelle F, Leboulanger $N$, Enjolras 0 , Harris R, Roger G, Garabedian EN. Role of propranolol in the therapeutic strategy of infantile laryngotracheal hemangioma. Int J Pediatr Otorhinolaryngol. 2009;73(8): 1168-1172

16. Buckmiller L, Dyamenahalli U, Richter GT. Propranolol for airway hemangiomas: case report of novel treatment. Laryngoscope. 2009;119(10):2051-2054

17. Manunza F, Syed S, Laguda B, et al. Propranolol for complicated infantile haemangiomas: a case series of 30 infants. Br J Dermatol. 2010;162(2):466-468

18. Tan ST, Itinteang T, Leadbitter P. Low-dose propranolol for infantile haemangioma. J Plast Reconstr Aesthet Surg. 2010; In press. doi:10.1016/j.bjps.2010.06.010

19. Zarem HA, Edgerton MT. Induced resolution renin-angiotensin system. ${ }^{30}$ We propose that the renin-angiotensin system can account for both the action of $\beta$-blockers and the process of spontaneous involution of $\mathrm{IH}$, and we speculate the possibility of using inhibitors of angiotensin-converting enzyme (ACE) and that of angiotensin II receptor 2 as potential alternative therapies. ${ }^{29,30}$ Our vascular anomalies center has begun a clinical trial using an ACE inhibitor. Preliminary results of our study have been promising but are the subject of a separate report.

of cavernous hemangiomas following prednisolone therapy. Plast Reconstr Surg. 1967:39(1):76-83

20. Huang SA, Tu HM, Harney JW, Venihaki M, Butte AJ, Kozakewich HP. Severe hypothyroidism caused by type 3 iodothyronine deiodinase in infantile hemangiomas. $N$ Engl J Med. 2000;343(3):185-189

21. Edgerton MT. The treatment of hemangiomas: with special reference to the role of steroid therapy. Ann Surg. 1976;183(5):517530

22. Fost NC, Esterly NB. Successful treatment of juvenile hemangiomas with prednisone. $J$ Pediatr. 1968;72(3):351-357

23. Bennett ML, Fleischer AB Jr, Chamlin SL, Frieden IJ. Oral corticosteroid use is effective for cutaneous hemangiomas: an evidence-based evaluation. Arch Dermatol. 2001;137(9):1208-1213

24. Fawcett SL, Grant I, Hall PN, Kelsall AWR, Nicholson JC. Vincristine as a treatment for a large haemangioma threatening vital functions. Br J Plast Surg. 2004;57(2):168-171

25. Wharton S, SoueidA, Nishikawa H, SridharA. Endangering cutaneous infantile hemangioma treated with vincristine: a case report. Eur J Plast Surg. 2009;32(3):157-161

26. Honey M, Chamberlain DA, Howard J. The effect of beta-sympathetic blockade on arterial oxygen saturation in Fallot's tetralogy. Circulation. 1964;30:501-510

27. Cherian G, Brockington I, Shah P, Oakley C, Goodwin J. Beta-adrenergic blockade in hypertrophic obstructive cardiomyopathy. $B r$ Med J. 1966;1(5492):895-898

28. Siegfried EC, Keenan WJ, Al-Jureidini S, Leaute-Labreze C, de la Roque ED, Taieb A. More on propranolol for hemangiomas of infancy. N Engl J Med. 2008;359(26): 2846-2847 
29. Tan ST, Itinteang T, Leadbitter P. Low dose propranolol treatment for infantile haemangioma. J Plast Reconstr Aesthet Surg. 2010; In press

30. Itinteang T, Brasch HD, Tan ST, Day DJ. Expression of components of the reninangiotensin system in proliferating infantile haemangioma may account for the propranolol-induced accelerated involution. J Plast Reconstr Aesth Surg. 2010; In press. doi:10.1016/j.bjps. 2010.08.039

31. North PE, Waner M, Mizeracki A, et al. A unique microvascular phenotype shared by juvenile hemangiomas and human placenta. Arch Dermatol. 2001;137(5):559-570 32. Itinteang T, Tan ST, Brasch H, Day DJ.
Haemogenic endothelium in infantile haemangioma. J Clin Pathol. 2010;63(11): 982-986

33. Itinteang T, Tan ST, Brasch H, Day DJ. Primitive mesodermal cells with a neural crest stem cell phenotype predominate proliferating infantile haemangioma. J Clin Pathol. 2010;63(9):771-776

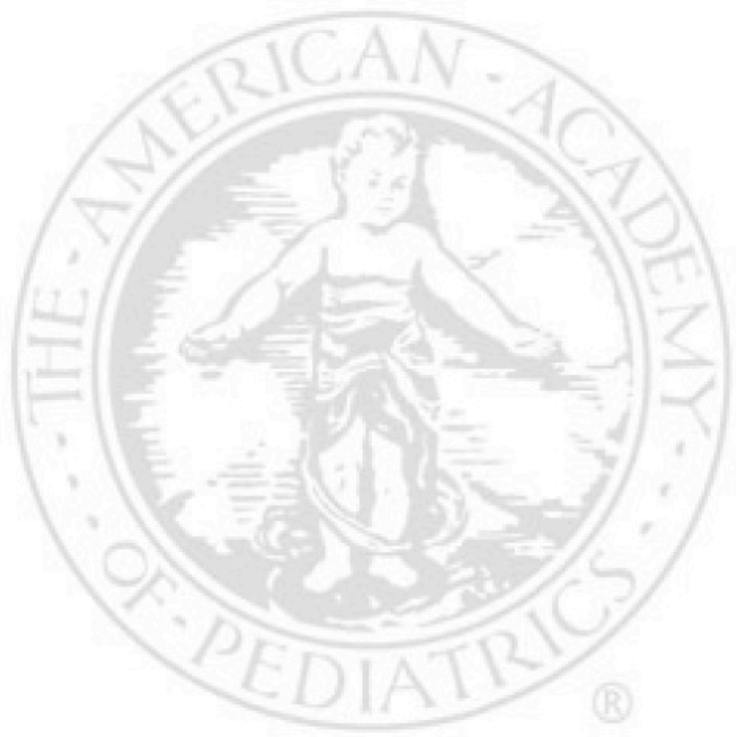


11.5. Manuscript: Pharmacologic Therapies For Infantile Hemangioma: Is There A Rational Basis? 


\section{Pharmacologic Therapies for Infantile Hemangioma: Is There a Rational Basis?}

\begin{tabular}{|c|c|}
\hline $\begin{array}{r}\text { Tinte Itinteang, M.B.B.S. } \\
\text { Aaron H. J. Withers, } \\
\text { M.B.Ch.B. } \\
\text { Philip Leadbitter, M.B.Ch.B. } \\
\text { Darren J. Day, Ph.D. } \\
\text { Swee Thong Tan, M.B.B.S., } \\
\text { Ph.D. } \\
\text { Wellington, New Zealand }\end{array}$ & $\begin{array}{l}\text { Background: Infantile hemangioma is the most common tumor of infancy. The } \\
\text { majority of cases are managed conservatively, but intervention is necessary in } \\
\text { approximately } 10 \text { percent of cases because of the threat to life or function or } \\
\text { because of tissue distortion or destruction. The mainstay treatment for these } \\
\text { problematic proliferating infantile hemangiomas is pharmacologic therapy, } \\
\text { mostly discovered serendipitously. } \\
\text { Methods: This review examines the rational basis of the hitherto empirical } \\
\text { pharmacologic therapies for the enigmatic infantile hemangioma, in light of } \\
\text { new knowledge regarding its biology, including the critical roles of stem cells } \\
\text { and the renin-angiotensin system. } \\
\text { Results: Steroids have remained the first-line therapy for problematic infantile } \\
\text { hemangioma for over } 40 \text { years despite their known side effects and failure rates. } \\
\text { Vincristine has emerged as an alternative to interferon for steroid-resistant cases } \\
\text { because of interferon's adverse effects, especially neurotoxicity. } \beta \text {-Blockers are } \\
\text { now the preferred first-line therapy for problematic cases. There is increasing } \\
\text { evidence that infantile hemangioma is a disorder of aberrant proliferation and } \\
\text { differentiation of primitive mesoderm-derived neural crest phenotypic cells. } \\
\text { This primitive phenotype that gives rise to a hemogenic endothelium interme- } \\
\text { diate has the ability to undergo primitive erythropoiesis and terminal mesen- } \\
\text { chymal differentiation. } \\
\text { Conclusions: The recent discovery of the crucial role of stem cells and the } \\
\text { inferred role of the renin-angiotensin system in the biology of infantile hem- } \\
\text { angioma underscores the possibility of even more targeted therapies, by using } \\
\text { modulators of the renin-angiotensin system, on infantile hemangioma. The } \\
\text { observation of the potential role of these traditional antihypertensive agents in } \\
\text { stem cell biology may lead to better understanding of developmental biology } \\
\text { and tumor stem cell growth. (Plast. Reconstr. Surg. } 128 \text { : } 499 \text {, } 2011 \text {.) }\end{array}$ \\
\hline
\end{tabular}

nfantile hemangioma, the most common tumor of infancy, affects 10 percent of infants, with

a female-to-male ratio of $3: 1$ and a preponderance in premature infants and Cauasians. ${ }^{1-4}$ Infantile hemangioma is characterized by rapid growth during infancy (proliferating phase), typified by excessive angiogenesis. This is inevitably followed by spontaneous involution over 1 to 5 years (involuting phase) when the cellular elements are

From the School of Biological Sciences, Victoria University of Wellington; the Center for the Study and Treatment of Vascular Birthmarks, Wellington Regional Plastic, Maxillofacial, and Burns Unit; the Gillies McIndoe Research Institute; the Department of Pediatrics, Hutt Hospital; and the University of Otago.

Received for publication December 18, 2010; accepted February 15,2011

Copyright $(\mathrm{C} 2011$ by the American Society of Plastic Surgeons DOI: 10.1097/PRS.0b013e31821b63a0 gradually replaced by fibrofatty deposition with continued improvement up to the age of 12 years (involuted phase). ${ }^{1-3,5}$ Proliferating infantile hemangioma is composed of a heterogeneous population of cells of different lineages, predominantly immature endothelial cells,,$^{2,3}$ and a lesser abundance of cells of the myeloid ${ }^{6,7}$ and mesenchymal lineages. 8,9

There is increasing evidence that infantile hemangioma is a disorder of aberrant proliferation and differentiation of primitive mesoderm-derived neural crest phenotypic cells. ${ }^{10}$ This primitive phenotype that gives rise to a he-

Disclosure: The authors have no financial interest in any of the products, devices, or drugs mentioned in this article. 
mogenic endothelium intermediate ${ }^{11}$ with the ability to undergo primitive erythropoiesis ${ }^{12}$ and terminal mesenchymal differentiation ${ }^{9}$ is potentially governed by the renin-angiotensin system. ${ }^{13}$ The identification of this primitive population within proliferating infantile hemangioma infers a possible embryonic stem cell origin for infantile hemangioma. ${ }^{10,14}$

This article reviews current pharmacologic therapies for problematic proliferating infantile hemangioma. We present the increasing evidence of various cell surface receptors and cytokines governing embryonic stem cell-derived primitive mesoderm differentiation pathways. Our data provide some insights into the mechanism of action of current therapeutic agents that explain and rationalize their observed clinical responses.

\section{GLUCOCORTICOID THERAPY}

Glucocorticoids were serendipitously discovered to induce accelerated regression of proliferating infantile hemangioma in the $1960 \mathrm{~s}^{15,16}$ and have remained the mainstay treatment for problematic proliferating infantile hemangioma for over 40 years. ${ }^{17}$ There are two forms of glucocorticoid therapy. High-dose systemic oral prednisone (or an equivalent dose of prednisolone) is administered typically at 2 to $3 \mathrm{mg} / \mathrm{kg}$ /day, usually as a single dose $\mathrm{e}^{17,18}$ over an average duration of 2 months, ${ }^{19}$ followed by a rapid taper over 2 months. ${ }^{17}$ Intralesional corticosteroid administration is preferred for localized nonperiorbital lesions. Intralesional triamcinolone acetonide is usually administered singly, ${ }^{18}$ although it has been used in combination with dexamethasone sodium phosphate for high-risk lesions. ${ }^{18}$ Injections usually consist of $20 \mathrm{mg}$ of triamcinolone acetonide and $3 \mathrm{mg}$ of betamethasone in $1 \mathrm{ml}$ total volume, ${ }^{20}$ although there have been other reports of this dose being doubled. ${ }^{21} \mathrm{~A}$ weight-based protocol of up to 3 to $5 \mathrm{mg} / \mathrm{kg}$ per injection has also been used and different selection criteria, an overall good response rate of 35 to 90 percent to intralesional steroid therapy has been reported. ${ }^{23}$

The side effects of systemic glucocorticoid therapy are well documented ${ }^{17,22}$ and are relatively common, even for short treatment periods. These include cushingoid facies, weight gain, hypertension, cataracts, glucose intolerance, and adrenal suppression. Growth retardation is more common if treatment is started before 3 months of age or continued for 6 months or longer, presumably because of decreased somatomedin and therefore collagen synthesis. However, accelerated catchup growth occurs, reassuming pretreatment growth, following cessation of systemic steroid therapy. ${ }^{17,22}$ There is also increasing evidence of reduced brain growth with steroid use in premature infants. ${ }^{25}$ Intralesional steroid therapy is associated with fewer systemic side effects compared with systemic administration. ${ }^{20-22}$ However, reports of central retinal artery occlusion following steroid injection of periorbital infantile hemangiomas has been reported. ${ }^{26}$

The demonstration of mesenchymal stem cells in infantile hemangioma ${ }^{8}$ and the presumed mesenchymal/mesodermal ${ }^{27}$ origin of infantile hemangioma has led to the assumption that these cells through differentiation form a crucial role in the formation of the fibrofatty residuum, typically seen in involuted lesions. The recent discovery of a neural crest phenotypic precursor in proliferating infantile hemangioma that coexpresses mesenchyme-associated cell proteins ${ }^{10}$ has led us to propose that these neural crest phenotypic cells have a mesenchymal and thus adipocytic differentiation capacity, corroborating previous reports. ${ }^{28,29}$

Glucocorticoids have been implicated in the acceleration, but not the initiation, of adipocytic differentiation. ${ }^{30}$ The effect of glucocorticoids on promoting apoptosis by means of up-regulation of the mitechondrial cytochrome $b$ gene $e_{-}^{31}$ but not

\begin{tabular}{|c|c|c|}
\hline & 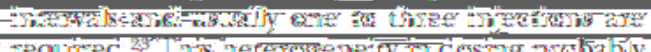 & 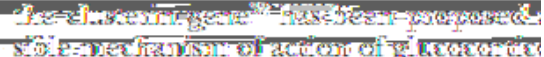 \\
\hline $\begin{array}{l}\text { Me efer: } \\
\text {-uedear: }\end{array}$ & 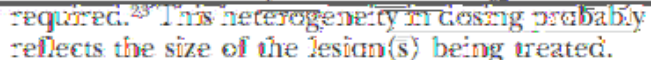 & 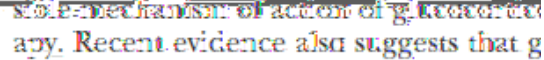 \\
\hline threvigh & Over the past four decades, several studies & tice \\
\hline ibitor of & have reportec variable effec & inh \\
\hline nstrated & therapy en infantle hemang:ena, with a dramate & acii \\
\hline $\begin{array}{l}\text { endo- } \\
\text { igioma }\end{array}$ & response cccurring in 30 to 53 percent of cases, & $\begin{array}{l}\text { expression of preadipocyte fact } \\
\text { thelium of proliferating infan: }\end{array}$ \\
\hline rolutes, ${ }^{9}$ & cases, and ongoing progression occurring in the & capillaries that is lost as the \\
\hline critical & remainder. ${ }^{17,19,24} \mathrm{~A}$ meta-analysis shows a 14 to 37 & possible mech: \\
\hline nd prea- & percent rate of rebound growth during dose & g. \\
\hline & $\begin{array}{l}\text { tapering, }{ }^{19} \text { often requiring reinstitution of ste- } \\
\text { roids. Despite the wide variations in the dosages }\end{array}$ & of infantile hemangioma. \\
\hline
\end{tabular}


Volume 128, Number 2 • Pharmacotherapy for Infantile Hemangioma

Another possible action of glucocorticoids involves its effect on peroxisome proliferator-activated receptor- $\gamma$, a transcription factor regulating adipocyte differentiation from preadipocytes. ${ }^{34}$ Glucocorticoids have been shown to induce peroxisome proliferator-activated receptor- $\gamma$ gene expression during conversion of mesenchymal stem cells into adipocytes. ${ }^{35}$ Considered together, we speculate that glucocorticoids exert a dual effect by negating the antiadipocytic differentiation effect of preadipocyte factor- 1 and promoting adipogenesis through increasing the expression of peroxisome proliferator-activated receptor- $\gamma$. Recent evidence has shown a critical role for angiotensin II, not only in proliferation of infantile hemangioma capillaries but also in the maintenance of vascular endothelial growth factor (VEGF) secretion by mesenchymal stem cells and preventing terminal adipocytic differentiation during the proliferation phase of infantile hemangioma. ${ }^{13}$ These observations have been supported by recent data from Greenberger et al. ${ }^{36}$ demonstrating a role for corticosteroids in suppressing VEGF-A secretion in infantile hemangioma-derived stem cells. ${ }^{36}$ These authors suggest a direct inhibitory effect of dexamethasone on vasculogenesis. The relative effects of steroids on adipogenesis and vasculogenesis remain a topic for further investigation. We propose that these mechanisms account for the variable clinical response of infantile hemangioma to glucocorticoid therapy with respect to the stage of differentiation of infantile hemangioma and the time point at which treatment is initiated.

\section{INTERFERON THERAPY}

During investigation into the antiviral agent interferon $\alpha$-2a for the treatment of human immunodeficiency virus, it was noted that there was improvement of the patients' Kaposi sarcomas. ${ }^{37-39}$ Anecdotal reports on the response of infantile hemangioma to interferon ${ }^{40,41}$ and subsequent clinical trials ${ }^{42,43}$ have led to its use as a second-line treatment for steroidresistant infantile hemangiomas. ${ }^{44}$

Two forms of interferon therapy are used for problematic proliferating infantile hemangioma, namely, interferon $\alpha$-2a and interferon $\alpha$-2b. Interferon $\alpha-2 \mathrm{a}$ is administered as daily subcutaneous injections at a starting dose of 1 million $\mathrm{IU} / \mathrm{m}^{2}$ body surface area, increasing to 3 million $\mathrm{IU} / \mathrm{m}^{2}$ during the first month of treatment, for a period of 2 to 12 months. ${ }^{41,45,46}$ The response rate to interferon is 90 percent in steroid-resistant infantile hemangiomas. However, the response is less dramatic and takes longer than steroid therapy. ${ }^{44}$ Pre- mature withdrawal of interferon is associated with rebound growth, necessitating reintroduction of the treatment.

Side effects of interferon therapy include fever, flu-like symptoms, rash, gastrointestinal symptoms, transient neutropenia, and elevated liver enzymes. ${ }^{44,46,47}$ The most serious complication of interferon therapy is neurotoxicity affecting mainly higher cortical function and, to a lesser extent, motor function. ${ }^{44}$ There have also been reports of peripheral neuropathy. ${ }^{44}$ These side effects are usually reversible following cessation of therapy. The most serious neurotoxic side effect of interferon is spastic diplegia, which affects up to 20 percent of patients. ${ }^{48}$ Of concern are reported cases of persistent spastic diplegia ${ }^{49}$ despite cessation of interferon $\alpha$-2a. The underlying mechanism of interferon toxicity is unknown but appears to be related to the duration of treatment and not the maximal daily dosage, as they appear late during treatment. Regular monitoring with neurologic assessment, full blood count, and liver function testing should be performed during treatment.

The successful use of topical imiquimod ${ }^{50,51}$ in treating proliferating infantile hemangioma, has been attributed to its ability to induce interferon $\alpha$ production, ${ }^{50,51}$ suggesting a common final pathway in the observed effect of interferon and imiquimod therapy.

Interferon-induced genes have been shown to be up-regulated during the involuting phase of infantile hemangioma. ${ }^{52}$ Thus, the induction of these genes provides the basis of interferon therapy. Interferon $\alpha$-2a inhibits the locomotion of endothelial cells in vitro, inhibits fibroblast growth factor-2, and has been shown to inhibit angiogenesis. ${ }^{53,54}$ It has also been shown that the epidermis overlying proliferating infantile hemangioma expresses less interferon $\beta$ than the normal epidermis. ${ }^{55}$ This may indicate a role for paracrine effects of nonendothelial cells in the pathogenesis of infantile hemangioma. Further work with in vitro human model ${ }^{56}$ may allow the study of its precise mechanism of action.

Although interferon $\alpha$-2a is effective in treating massive or life-threatening steroid-resistant infantile hemangiomas, its potential serious side effects have limited its use, and vincristine has emerged as an alternative second-line therapy. Because of its toxicities and the emergence of more effective therapeutic options, interferon should be used as a last-resort therapy for problematic infantile hemangiomas. 


\section{VINCRISTINE THERAPY}

Vincristine belongs to the vinca alkaloids class of drugs, which act predominantly as inhibitors of mitosis. ${ }^{57}$ The use of vincristine for adult and pediatric malignancies is well documented. Its application in infantile hemangioma, although limited, has been documented in life-threatening steroid- and/or interferon-resistant infantile hemangiomas. ${ }^{58,59}$

The dosage of vincristine for infantile hemangioma is $0.05 \mathrm{mg} / \mathrm{m}^{2}$ in infants weighing less than $10 \mathrm{~kg}$, and 0.75 to $1.5 \mathrm{mg} / \mathrm{m}^{2}$ for infants weighing more than $10 \mathrm{~kg}$, given intravenously through a central venous line once weekly for three to four doses, ${ }^{45,58-61}$ with dosing intervals varying 1 to 3 weeks.

Side effects of vincristine include neurotoxicity, with irritability, loss of deep tendon reflexes, constipation, abdominal pain, paralytic ileus, cranial nerve palsies, and bone pain (commonly of the jaw) reported. ${ }^{61}$ Neurotoxicity is usually not as marked in children or infants, as observed in adults. Other side effects include alopecia, rash and, uncommonly, myelosuppression. ${ }^{61}$ Vincristine is a vesicant, and the risk of extravasation necessitates administration through a central venous line or cautious administration through a peripheral line. Supervision by a pediatric oncologist with regular neurologic examination with full blood count during treatment is essential. ${ }^{60}$

Vincristine inhibits angiogenesis by preventing cell proliferation through inhibition of cell mitosis ${ }^{57}$ This occurs at metaphase by preventing tubulin polymerization and thus not allowing microtubule formation, and the induction of microtubule depolymerization leading to Bcl-2 phosphorylation, resulting in loss of its ability to form heterodimers with Bax leading to eventual induction of cellular apoptosis. ${ }^{62,63}$ It is interesting that during involution of infantile hemangioma the levels of Bcl-2 start to decrease, with a concomitant increase in Bax levels, allowing for an increase in Bax homodimerization with subsequent cellular apoptosis ${ }^{64}$ Experimental models have shown favorable results with lower dosing of vincristine when given concomitantly with an angiogenic inhibitor, suggesting a critical role of vincristine in inducing terminal apoptotic pathways in compromised cells. ${ }^{65}$

\section{$\beta$-BLOCKER THERAPY}

The recent serendipitous discovery of accelerated involution of infantile hemangioma induced by propranolol, ${ }^{66}$ a nonselective $\beta$-blocker; and acebutalol, ${ }^{67}$ a predominantly $\beta_{1}$-adrenoreceptor blocker, ${ }^{68}$ has resulted in a significant paradigm shift in the treatment of problematic proliferating infantile hemangioma. Propranolol is now the preferred treatment for problematic proliferating infantile hemangioma. ${ }^{69-75}$ The mechanism of action of $\beta$-blockers on proliferating infantile hemangioma is unknown, although their effects on $\beta$-adrenergic receptor stimulation, ${ }^{68}$ down-regulation of angiogenic growth factors, ${ }^{6,76,77}$ reduction in expression of matrix metalloprotinases, ${ }^{77}$ and induction of apoptosis ${ }^{78}$ have been suggested.

Most reports on propranolol therapy use a 2to $3-\mathrm{mg} / \mathrm{kg} /$ day dosing regimen given in two to three divided doses, ${ }^{69-71,79}$ with treatment courses ranging from 6 weeks ${ }^{6}$ to 7 months ${ }^{71}$ usually with a 24-hour hospital stay during initiation of therapy and regular hemodynamic monitoring during the first 6 hours of treatment. ${ }^{70}$ We have recently shown that low-dose propranolol at $1.5 \mathrm{mg} / \mathrm{kg} /$ day is sufficient to induce accelerated involution of infantile hemangioma in the majority of patients (Fig. 1), with a small subgroup, all female patients, requiring a dosage of $2 \mathrm{mg} / \mathrm{kg} /$ day. ${ }^{73,80}$

The dosage of acebutolol in the treatment of infantile hemangioma is 8 to $10 \mathrm{mg} / \mathrm{kg} /$ day, with clinical responses observed 1 month after initiation of treatment. ${ }^{67,81}$ The optimal duration of treatment remains to be determined. Topical $\beta$-blockers such as timolol, a nonselective $\beta$-blocker, administered either by two drops of $0.5 \%$ timolol maleate ophthalmic solution ${ }^{82}$ or as a gel preparation applied twice daily directly onto the infantile hemangioma, ${ }^{83}$ have shown promising results.

Although $\beta$-blockers have a long and good safety record in the treatment of young children with hypertension and other cardiovascular diseases ${ }^{84}$ this may not be simply extrapolated to infants (some of whom are born prematurely) and young children with infantile hemangioma, who are otherwise normotensive. ${ }^{80}$ The potential adverse effects of propranolol include bradycardia, hypotension, and hypoglycemia, ${ }^{85}$ with recent reports highlighting symptomatic and potentially fatal hypoglycemia in this patient cohort. ${ }^{86,87} \mathrm{Cau}-$ tion has justifiably been raised regarding the casual use of this group of drugs for this novel indication in an otherwise normotensive population. ${ }^{80,85}$ The need for large multicenter trials, such as the one currently in progress, ${ }^{85}$ will possibly define the clinical indications and appropriate treatment protocols for this condition.

The identification of angiotensin-converting enzyme as a cell surface protein associated with the 
Volume 128, Number 2 • Pharmacotherapy for Infantile Hemangioma

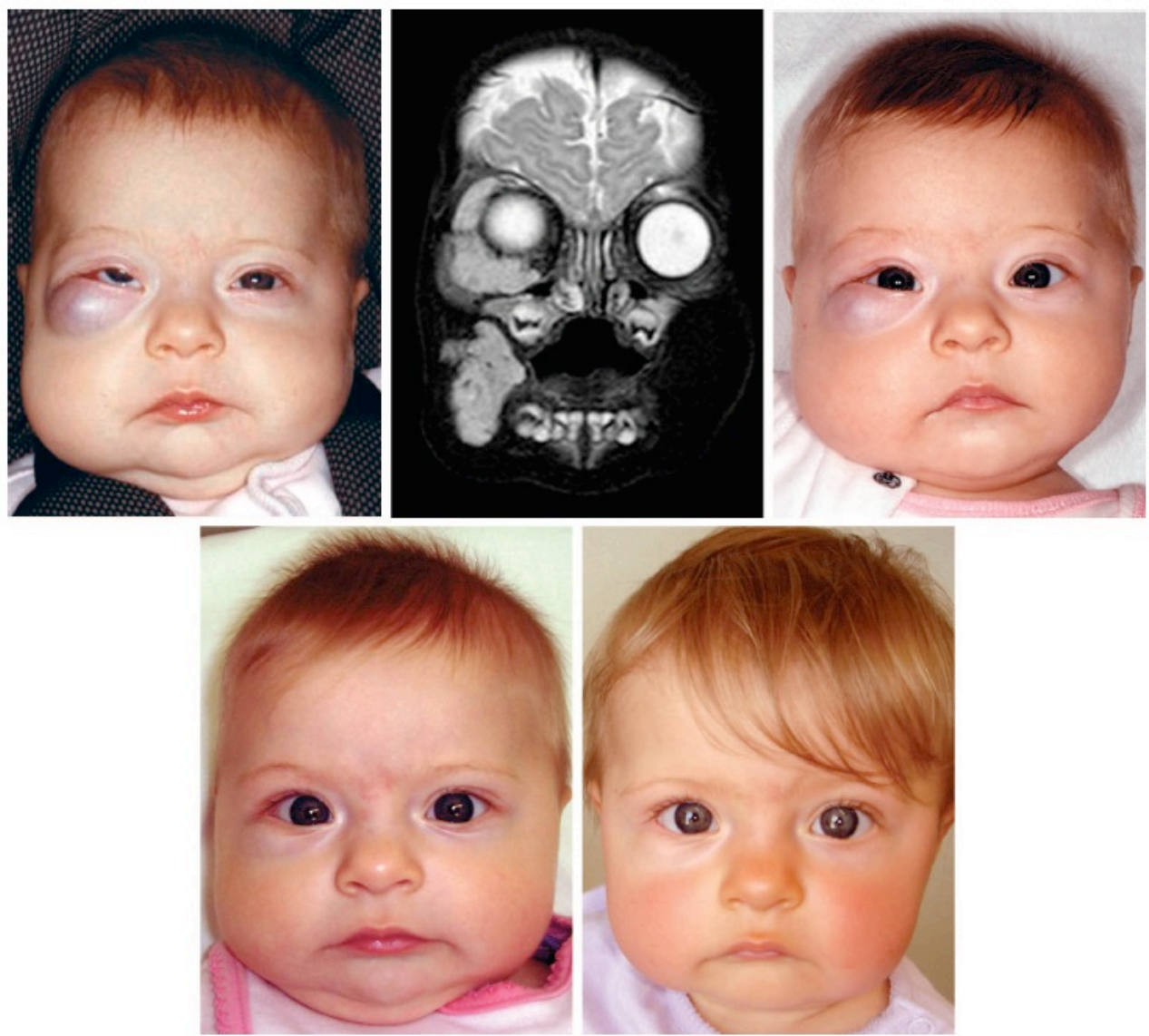

Fig. 1. (Above, left) A 4-month-old girl presented with a rapidly growing hemangioma on the right cheek, lower lid, and orbit with ocular dystopia, as seen on a T2-weighted magnetic resonance imaging scan (above, center). Accelerated involution of the hemangioma with equalization of the globe (above, right) 7 days, (below, left) 4 weeks, and (below, right) 5 months after institution of propranolol therapy at $2 \mathrm{mg} / \mathrm{kg} / \mathrm{day}$.

hemangioblast ${ }^{88}$ has led us to discover the expression of this protein on the immature capillaries of proliferating infantile hemangioma. ${ }^{13}$ We have also demonstrated the expression of the angiotensin receptor 2 on these same capillaries. ${ }^{13}$

The presence of both angiotensin-converting enzyme and angiotensin receptor 2 within proliferating infantile hemangioma suggests that the local angiotensin II produced by the conversion of angiotensin I by angiotensin-converting enzyme acts in an autocrine/paracrine fashion. This coupled with the observation of increased renin levels in premature ${ }^{89}$ female $^{90}$ and Caucasian infants ${ }^{91}$ corresponding to a higher incidence of infantile hemangioma in these groups of patients ${ }^{2}$ has led us to investigate the role of the renin-angiotensin system in infantile hemangioma. ${ }^{13}$

Angiotensin II causes secretion of VEGF from mesenchymal stem cells, ${ }^{92}$ which are present within infantile hemangioma, ${ }^{8}$ and proliferation of CD $34^{+}$cells ${ }^{93}$ Angiotensin II has also been reported to prevent terminal differentiation of mesenchymal stem cells. ${ }^{94}$ Considered together, it is exciting to speculate that angiotensin II drives proliferation of the immature endothelial cells, which express CD $34,{ }^{95}$ within proliferating infan- 
Plastic and Reconstructive Surgery • August 2011

tile hemangioma, and maintain continual VEGF production from the mesenchymal stem cells within the lesion, ${ }^{8}$ preventing terminal adipocytic differentiation, although this remains to be determined conclusively. The effect of angiotensin II on angiotensin receptor 2 has been demonstrated to cause an increase in Pax-2 expression in mouse embryonal mesenchymal epithelial cells. ${ }^{96}$ Pax-2 is a homeobox 2 gene associated with progenitor cells derived from embryonic stem cells ${ }^{97}$ and in mesenchymal condensates involved in early renal organogenesis ${ }^{98}$ which is lost as progenitor cells differentiate. ${ }^{97,98}$ The demonstration of Wnt activation by $P a x-2$, coupled with the knowledge of prevention of terminal adipocytic differentiation by Wnt ${ }^{99}$ may reveal novel pathways in adipogenic ontogeny in infantile hemangioma. The implication of a VEGF-notch signaling pathway in proliferating infantile hemangioma has also been demonstrated, ${ }^{100}$ and the effect of notch signaling on progenitor cell proliferation is reliant on normal Wnt signaling. ${ }^{101}$ Interestingly, Wnt signaling is involved not only in blocking apoptosis but also in preventing terminal differentiation of preadipocytes. ${ }^{99}$

The identification of osteoprotegerin, a secreted glycoprotein, belonging to the tumor necrosis factor receptor superfamily ${ }^{102}$ within proliferating infantile hemangioma ${ }^{103,104}$ also reveals a novel antiapoptotic pathway in infantile hemangioma. Osteoprotegerin acts to stimulate cell survival through its action as a receptor for tumor necrosis factor-related apoptosis ligand, by binding to tumor necrosis factor-related apoptosis ligand and preventing its interaction with functional death receptors, therefore preventing apoptosis. ${ }^{102}$ Angiotensin II has also been previously reported to stimulate osteoprotegerin production. ${ }^{105}$

It is known that $\beta$-blockers, such as propranolol, partly reduce renin activity in the periphery by reducing plasma renin activity. ${ }^{106}$ Low renin activity in turn reduces angiotensin I and eventually angiotensin II levels. Considered together, we infer that a reduction in angiotensin II levels causes a reduction in proliferation of the hemogenic endothelium of proliferating infantile hemangioma together with an increased rate of cellular apoptosis through the tumor necrosis factor-related apoptosis ligand pathway. A reduction in renin levels that physiologically occurs after the first year of life ${ }^{107}$ and the inferred mechanism of action of propranolol through the renin-angiotensin system is thus able to account for both the effect of $\beta$-blockers on infantile hemangioma and the natural progression of infantile hemangioma. It is intriguing that although the high systemic levels of renin during infancy may account for the rapid proliferation of infantile hemangioma, there is also the possibility of a local renin-angiotensin system including local secretion of renin as observed in the vasculature and adipose tissue $\mathrm{e}^{108}$ and from mast cells, ${ }^{109}$ which may contribute to the available angiotensin II to the tumor, which may explain the observed response of infantile hemangiomas to topical application of timolol. ${ }^{82,83}$ However, this remains a topic of further investigation.

\section{THE FUTURE}

The recent discovery of the crucial role of stem cells ${ }^{10}$ and the inferred role of the renin-angiotensin system ${ }^{13}$ in the biology of infantile hemangioma underscores the possibility of using even more targeted therapy on this enigmatic condition. Our preliminary clinical trial using an angiotensin-converting enzyme inhibitor has shown promising results, ${ }^{80}$ although this is a subject for a separate report. This has raised the possibility for the novel use of inhibitors of angiotensin-converting enzyme and angiotensin receptor as modulators of the renin-angiotensin system in the treatment of infantile hemangioma. The novel vasopeptidase inhibitors that simultaneously inhibit neutral endopeptidase and serum angiotensin-converting enzyme activity ${ }^{110}$ may also offer potential reninangiotensin system modulation. Further study will be needed to verify the potential role of these modulators of the renin-angiotensin system in the treatment of infantile hemangioma. The discovery of the potential role of these traditional antihypertensive agents in stem cell biology may lead to better understanding of developmental biology and tumor stem cell growth.

$$
\begin{array}{r}
\text { Swee Thong Tan, M.B.B.S., Ph.D. } \\
\text { Wellington Regional Plastic } \\
\text { Maxillofacial and Burns Unit } \\
\text { Hutt Hospital, Private Bag 31-907 } \\
\text { High Street } \\
\text { Lower Hutt, Wellington, New Zealand } \\
\text { swee.tan@huttvalleydhb.org.nz }
\end{array}
$$

Parents or guardians provided written consent for the use of the patient images.

1. Tan ST, Velickovic M, Ruger BM, Davis PF. Cellular and extracellular markers of hemangioma. Plast Reconstr Surg. 2000;106:529-538.

2. Takahashi K, Mulliken JB, Kozakewich HP, Rogers RA, Folkman J, Ezekowitz RA. Cellular markers that distinguish the phases of hemangioma during infancy and childhood.J Clin Invest. 1994;93:2357-2364. 
Volume 128, Number 2 - Pharmacotherapy for Infantile Hemangioma

3. Dosanjh A, Chang J, Bresnick S, et al. In vitro characteristics of neonatal hemangioma endothelial cells: Similarities and differences between normal neonatal and fetal endothelial cells. J Cutan Pathol. 2000;27:441-450.

4. North PE, Waner M, Mizeracki A, Mihm MC Jr. Glutl: A newly discovered immunohistochemical marker for juvenile hemangiomas. Human Pathol. 2000;31:11-22.

5. Mulliken JB, Glowacki J. Hemangiomas and vascular malformations in infants and children: A classification based on endothelial characteristics. Plast Reconstr Surg. 1982 69:412-422.

6. Ritter MR, Reinisch J, Friedlander SF, Friedlander M. Myeloid cells in infantile hemangioma. Am J Pathol. 2006;168: $621-628$.

7. Tan ST, Wallis RA, He Y, Davis PF. Mast cells and hemangioma. Plast Reconstr Surg. 2004;113:999-1011.

8. Yu Y, Fuhr J, Boye E, et al. Mesenchymal stem cells and adipogenesis in hemangioma involution. Stem Cells 2006; 24:1605-1612.

9. Itinteang T, Vishvanath A, Day DJ, Tan ST. Mesenchymal stem cells in infantile haemangioma. J Clin Pathol. 2011;64 232-236.

10. Itinteang T, Tan ST, Brasch H, Day DJ. Primitive mesodermal cells with a neural crest stem cell phenotype predominate proliferating infantile haemangioma. I Clin Pathol 2010;63:771-776.

11. Itinteang T, Tan ST, Brasch H, Day DJ. Haemogenic endothelium in infantile haemangioma. J Clin Pathol. 2010 63:982-986

12. Itinteang T, Tan ST, Brasch HD, Vishvanath A, Day DJ. Primitive erythropoiesis in infantile haemangioma. Br J Dermatol. 2011;164:1097-1100.

13. Itinteang T, Brasch HD, Tan ST, Day DJ. Expression of components of the renin-angiotensin system in proliferating infantile haemangioma may account for the propranolol-induced accelerated involution. J Plast Reconstr Aesthet Surg. 2011;64:759-765.

14. Itinteang T, Tan ST, Brasch HD, et al. Infantile hemangioma expresses embryonic stem cell markers. Br J Dermatol. (in press).

15. Zarem HA, Edgerton MT. Induced resolution of cavernous hemangiomas following prednisolone therapy. Plast Reconstr Surg. 1967;39:76_83.

16. Fost NC, Esterly NB. Successful treatment of juvenile hemangiomas with prednisone. J Pediatr. 1968;72:351-357.

17. Boon LM, MacDonald DM, Mulliken JB. Complications of systemic corticosteroid therapy for problematic hemangisystemic corticosteroid therapy for problema
oma. Plast Reconstr Surg. 1999;104:1616-1623.

18. Frieden IJ, Eichenfield LF, Esterly NB, Geronemus R, Mallory SB. Guidelines of care for hemangiomas of infancy. American Academy of Dermatology Guidelines/Outcome Committee. J Am Acad Dermatol. 1997;37:631-637.

19. Bennett ML, Fleischer AB Jr, Chamlin SL, Frieden IJ. Oral corticosteroid use is effective for cutaneous hemangiomas: An evidence-based evaluation. Arch Dermatol. 2001;137: 1208-1213.

20. Sloan GM, Reinisch JF, Nichter LS, Saber WL, Lew K, Morwood DT. Intralesional corticosteroid therapy for infantile hemangiomas. Plast Reconstr Surg. 1989:83:495-467.

21. Kushner BJ. The treatment of periorbital infantile hemangioma with intralesional corticosteroid. Plast Reconstr Surg. 1985;76:517-526.

22. Tan BH, Leadbitter PH, Auburn NH, Tan ST. Steroid therapy for problematic proliferating haemangioma. NZ Med J. 2011;124:57-65
23. Chen MT, Yeong EK, Horng SY. Intralesional corticosteroid therapy in proliferating head and neck hemangiomas: A review of 155 cases. J Pediatr Surg. 2000;35:420-423.

24. Enjolras O, Riche MC, MerlandJJ, Escande JP. Management of alarming hemangiomas in infancy: A review of 25 cases. Pediatrics 1990:85:491-498.

25. Baud O. Postnatal steroid treatment and brain development. Arch Dis Child Fetal Neonatal Ed. 2004;89:F96-F100.

26. Shorr N, Seiff SR. Central retinal artery-occlusion associated with periocular corticosteroid injection for juvenile hemangioma. Ophthalmic Surg. 1986;17:229-231.

27. Boscolo E, Bischoff J. Vasculogenesis in infantile hemangioma. Angiogenesis 2009;12:197-207.

28. Billon N, Iannarelli P, Monteiro MC, et al. The generation of adipocytes by the neural crest. Development 2007;134: 2283-2292.

29. Lee G, Kim H, Elkabetz Y, et al. Isolation and directed differentiation of neural crest stem cells derived from human embryonic stem cells. Nat Biotechnol. 2007;25:14681475 .

30. Chapman AB, Knight DM, Ringold GM. Glucocorticoid regulation of adipocyte differentiation: Hormonal triggering of the developmental program and induction of a differentiation-dependent gene. J Cell Biol. 1985;101:12271235.

31. Hasan Q Tan ST, Gush J, Peters SG, Davis PF. Steroid therapy of a proliferating hemangioma: Histochemical and molecular changes. Pediatrics 2000;105:117-120.

32. Hasan Q, Tan S, Xu B, Davis PF. Effects of five commonly used glucocorticoids on haemangioma in vitro. Clin Exp Pharmacol Physiol. 2003;30:140-144.

33. Pantoja C, Huff JT, Yamamoto KR. Glucocorticoid signaling defines a novel commitment state during adipogenesis in vitro. Mol Biol Cell 2008;19:4032-4041.

34. Ameshima S, Golpon H, Cool CD, et al. Peroxisome proliferator-activated receptor gamma (PPARgamma) expression is decreased in pulmonary hypertension and affects endothelial cell growth. Circ Res. 2003;92:1162-1169.

35. Wu Z, Bucher N, Farmer SR. Induction of peroxisome proliferator-activated receptor gamma during the conversion of 3T3 fibroblasts into adipocytes is mediated by C/EBPbeta, C/EBPdelta, and glucocorticoids. Mol Cell Biol. 1996;16:4128-4136.

36. Greenberger S, Boscolo E, Adini I, Mulliken JB, Bischoff J. Corticosteroid suppression of VEGF-A in infantile hemangioma-derived stem cells. NEngl JMed. 2010;362:1005-1013.

37. Groopman JE, Gottlieb MS, Goodman J, et al. Recombinant alpha-2 interferon therapy for Kaposi's sarcoma associated with the acquired immunodeficiency syndrome. Ann Intern Med. 1984;100:671-676.

38. Real FX, Oettgen H, Krown SE. Kaposi's sarcoma and the acquired immunodeficiency syndrome: Treatment with high and low doses of recombinant leukocyte $\mathrm{A}$ interferon. J Clin Oncol. 1986;4:544-551.

39. Rios A, Mansell PW, Newell GR, Reuben JM, Hersh EM, Gutterman JU. Treatment of acquired immunodeficiency syndrome-related Kaposi's sarcoma with lymphoblastoid interferon. J Clin Oncol. 1985;3:506-512.

40. White CW, Sondheimer HM, Crouch EC, Wilson H, Fan LL. Treatment of pulmonary hemangiomatosis with recombinant interferon alfa-2a. N Engl J Med. 1989;320:1197-1200.

41. White CW, Wolf SJ, Korones DN, Sondheimer HM, Tosi MF, Yu A. Treatment of childhood angiomatous diseases with recombinant interferon alfa-2a. J Pediatr. 1991;118:59-66. 
42. Ezekowitz RA, Mulliken JB, Folkman J. Interferon alfa-2a therapy for life-threatening hemangiomas of infancy. NEngl J Med. 1992;326:1456-1463.

43. Ezekowitz RA, Mulliken JB, Folkman J. Interferon alfa-2b therapy for life-threatening hemangiomas of infancy. NEngl J Med. 1994:330:300.

44. Greinwald JH Jr, Burke DK, Bonthius DJ, Bauman NM, Smith RJ. An update on the treatment of hemangiomas in children with interferon alfa-2a. Arch Otolaryngol Head Neck Surg. 1999;125:21-27.

45. Chan YC, Giam YC Guidelines of care for cutaneous haemangiomas. Ann Acad Med Singapore 2005;34:117-123.

46. Jiménez-Hernández E, Dueñas-González MT, QuinteroCuriel JL, et al. Treatment with interferon-alpha-2b in children with life-threatening hemangiomas. Dermatol Surg. 2008:34:640_647.

47. Hastings MM, Milot J, Barsoum-Homsy M, Hershon L, Dubois J, Leclerc JM. Recombinant interferon alfa-2b in the treatment of vision-threatening capillary hemangiomas in childhood. J AAPOS. 1997;1:226-230.

48. Barlow CF, Priebe CJ, Mulliken JB, et al. Spastic diplegia as a complication of interferon alfa-2a treatment of hemangiomas of infancy. J Pediatr. 1998;132:527-530.

49. Wörle H, Maass E, Kōhler B, Treuner J. Interferon alpha-2a therapy in haemangiomas of infancy: Spastic diplegia as a severe complication. Eur J Pediatr. 1999;158:344.

50. Hazen PG, Carney JF, Engstrom CW, Turgeon KL, Reep MD, Tanphaichitr A. Proliferating hemangioma of infancy: Successful treatment with topical $5 \%$ imiquimod cream. Pediatr Dermatol. 2005;22:254-256.

51. Martinez MI, Sanchez-Carpintero I, North PE, Mihm MC Jr. Infantile hemangioma: Clinical resolution with $5 \%$ imiquimod cream. Arch Dermatol. 2002;138:881-884; discussion 884 .

52. Ritter MR, Dorrell MI, Edmonds J, Friedlander SF, Friedlander M. Insulin-like growth factor 2 and potential regulators of hemangioma growth and involution identified by large-scale expression analysis. Proc Natl Acad Sci USA. 2002; 99:7455-7460.

53. Heyns AD, Eldor A, Vlodavsky I, Kaiser N, Fridman R, Panet A. The antiproliferative effect of interferon and the mitogenic activity of growth factors are independent cell cycle events: Studies with vascular smooth muscle cells and endothelial cells. Exp Cell Res. 1985;161:297-306.

54. Feldman D, Goldstein AL, Cox DC, Grimley PM. Cultured human endothelial cells treated with recombinant leukocyte A interferon: Tubuloreticular inclusion formation, antiproliferative effect, and 2',5' oligoadenylate synthetase induction. Lab Invest. 1988;59:548-589.

55. Bielenberg DR, Bucana CD, Sanchez R, Mulliken JB, Folkman J, Fidler IJ. Progressive growth of infantile cutaneous hemangiomas is directly correlated with hyperplasia and angiogenesis of adjacent epidermis and inversely correlated with expression of the endogenous angiogenesis inhibitor, IFN-beta. Int J Oncol. 1999;14:401-408.

56. Tan ST, Hasan Q, Velickovic M, Rüger BM, Davis RP, Davis $\mathrm{PF}$. A novel in vitro human model of hemangioma. Mod Pathol. 2000;13:92_99.

57. Kung AL, Zetterberg A, Sherwood SW, Schimke RT. Cytotoxic effects of cell cycle phase specific agents: Result of cell cycle perturbation. Cancer Res. 1990;50:7307-7317.

58. Enjolras O, Brevière GM, Roger G, et al. Vincristine treatment for function- and life-threatening infantile hemangioma (in French). Arch Pediatr. 2004;11:99-107.
59. Perez Payarols J, Pardo Masferrer J, Gomez Bellvert C. Treatment of life-threatening infantile hemangiomas with vincristine. N Engl J Med. 1995;333:69.

60. Fawcett SL, Grant I, Hall PN, Kelsall AW, Nicholson JC. Vincristine as a treatment for a large haemangioma threatening vital functions. Br J Plast Surg. 2004;57:168-171.

61. Wharton S, Soueid A, Nishikawa H, Sridhar A. Endangering cutaneous infantile hemangioma treated with vincristine: A case report. Eur J Plast Surg. 2009;32:157-161.

62. Mabeta P, Pepper MS. A comparative study on the antiangiogenic effects of DNA-damaging and cytoskeletal-disrupting agents. Angiogenesis 2009;12:81-90.

63. Wang LG, Liu XM, Kreis W, Budman DR. The effect of antimicrotubule agents on signal transduction pathways of apoptosis: A review. Cancer Chemother Pharmacol. 1999;44: 355-361.

64. Yang H, Deng C, Shen S, Zhang D, Yu Y. Expression and significance of Bcl-2, Bax, Fas and caspace- 3 in different phases of human hemangioma. I Huazhong Univ Sci Tech nolog Med Sci. 2006;26:402-404.

65. Klement G, Baruchel S, Rak J, et al. Continuous low-dose therapy with vinblastine and VEGF receptor-2 antibody induces sustained tumor regression without overt toxicity. J Clin Invest. 2000;105:R15-R24.

66. Léauté-Labrèze $\mathrm{C}$, Dumas de la Roque $\mathrm{E}$, Hubiche T, Boralevi F, Thambo JB, Taieb A. Propranolol for severe hemangiomas of infancy. N Engl J Med. 2008;358:2649-2651.

67. Bigorre M, Van Kien AK, Valette H. Beta-blocking agent for treatment of infantile hemangioma. Plast Reconstr Surg. 2009; 123:195e-196e

68. Piña IL, Smith EV, Weidler DJ. Low-dose acebutolol given once daily in the treatment of chronic angina pectoris. JClin Pharmacol. 1988;28:427-430.

69. Buckmiller L, Dyamenahalli U, Richter GT. Propranolol for airway hemangiomas: Case report of novel treatment. $\mathrm{La}$. ryngoscope 2009;119:2051-2054.

70. Sans V, de la Roque ED, Berge J, et al. Propranolol for severe infantile hemangiomas: Follow-up report. Pediatrics 2009; 124:e423-e431.

71. Denoyelle F, Leboulanger N, Enjolras O, Harris R, Roger G, Garabedian E-N. Role of Propranolol in the therapeutic strategy of infantile laryngotracheal hemangioma. Int J Pe diatr Otorhinolaryngol. 2009;73:1168-1172.

72. Tan ST, Itinteang T, Leadbitter P. Low dose propranolol for multiple infantile hepatic and cutaneous hemangiomas with deranged liver function. Pediatrics 2011;127:e772e776.

73. Tan ST, Itinteang T, Leadbitter P. Low-dose propranolol for infantile haemangioma. J Plast Reconstr Aesthet Surg. 2011; 64:292-299.

74. Arneja JS, Pappas PN, Shwayder TA, et al. Management of complicated facial hemangiomas with beta-blocker (propranolol) therapy. Plast Reconstr Surg. 2010;126:889-895.

75. Holmes WJ, Mishra A, Gorst C, Liew SH. Propranolol as firstline treatment for rapidly proliferating infantile haemangiomas. J Plast Reconstr Aesthet Surg. 2011;64:445-451.

76. D'Angelo G, Lee H, Weiner RI. cAMP-dependent protein kinase inhibits the mitogenic action of vascular endothelial growth factor and fibroblast growth factor in capillary endothelial cells by blocking Raf activation. $J$ Cell Biochem. 1997;67:353-366.

77. Storch CH, Hoeger PH. Propranolol for infantile haemangiomas: Insights into the molecular mechanisms of action. Br J Dermatol. 2010;163:269-274.

506 
Volume 128, Number 2 • Pharmacotherapy for Infantile Hemangioma

78. Sommers Smith SK, Smith DM. Beta blockade induces apoptosis in cultured capillary endothelial cells. In Vitro Cell Deo Biol Anim. 2002;38:298-304.

79. Manunza F, Syed S, Laguda B, et al. Propranolol for complicated infantile haemangiom (Letter). A case series of 30 infants. Br J Dermatol. (in press)

80. Tan ST, Itinteang T, Leadbitter P. Low dose propranolol treatment for infantile haemangioma (Letter). J Plast Reconstr Aesthet Surg. (in press).

81. Blanchet C, Nicollas R, Bigorre M, Amedro P, Mondain M Management of infantile subglottic hemangioma: Acebutolol or propranolol? Int J Pediatr Otorhinolaryngol. 2010;74 959-961.

82. Guo S, Ni N. Topical treatment for capillary hemangioma of the eyelid using beta-blocker solution. Arch Ophthalmol. 2010;128:255-256.

83. Pope E, Chakkittakandiyil A. Topical timolol gel for infantile hemangiomas: A pilot study. Arch Dermatol. 2010;146 $564-565$

84. Artman M, Grayson M, Boerth RC. Propranolol in children Safety-toxicity, Pediatrics 1982:70:30-31

85. Siegfried EC, Keenan WJ, Al-Jureidini S. More on propranolol for hemangiomas of infancy. N Engl J Med. 2008;359: 2846; author reply 2846-2847.

86. Bonifazi E, Acquafredda A, Milano A, Montagna O, Laforgia N. Severe hypoglycemia during successful treatment of diffuse hemangiomatosis with propranolol. Pediatr Dermatol. 2010;27:195-196.

87. Holland KE, Frieden IJ, Frommelt PC, Mancini AJ, Wyatt D, Drolet BA. Hypoglycemia in children taking propranolol for the treatment of infantile hemangioma. Arch Dermatol 2010;146:775-778

88. Zambidis ET, Park TS, Yu W, et al. Expression of angiotensin-converting enzyme (CD143) identifies and regulates primitive hemangioblasts derived from human pluripotent stem cells. Blood 2008;112:3601-3614.

89. Stephenson TJ, Broughton Pipkin F, Elias-Jones AC. Factors influencing plasma renin and renin substrate in premature infants. Arch Dis Child. 1991;66:1150-1154.

90. Broughton Pipkin F, Smales OR, O'Callaghan M. Renin and angiotensin levels in children. Arch Dis Child. 1981;56:298-302.

91. Youmbissi TJ, Tedong F, Fairbank ST, Blackett-Ngu K, Mbede J. Plasma renin activity studies in a group of African neonates and children. J Trop Pediatr. 1990;36:128-130.

92. Shi RZ, Wang JC, Huang SH, Wang XJ, Li QP. Angiotensin II induces vascular endothelial growth factor synthesis in mesenchymal stem cells. Exp Cell Res. 2009;315:10-15.

93. Heringer-Walther S, Eckert K, Schumacher SM, et al. Angiotensin-(1-7) stimulates hematopoietic progenitor cells in vitro and in vivo. Haematologica 2009;94:857-860.
94. Matsushita K, Wu Y, Okamoto Y, Pratt RE, Dzau VJ. Local renin angiotensin expression regulates human mesenchymal stem cell differentiation to adipocytes. Hypertension 2006;48:1095-1102.

95. Smoller BR, Alfelberg DB. Infantile (juvenile) capillary hemangioma: A tumor of heterogeneous cellular elements. J Cutan Pathol. 1993;20:330-336.

96. Zhang SL, Moini B, Ingelfinger JR. Angiotensin II increases Pax-2 expression in fetal kidney cells via the AT2 receptor. J Am Soc Nephrol. 2004;15:1452-1465.

97. Li H, Roblin G, Liu H, Heller S. Generation of hair cells by stepwise differentiation of embryonic stem cells. Proc Natl Acad Sci USA. 2003;100:13495-13500.

98. Chi N, Epstein JA. Getting your Pax straight: Pax proteins in development and disease. Trends Genet. 2002;18:41-47.

99. Kennell JA, MacDougald OA. Wnt signaling inhibits adipogenesis through B-catenin-dependent and -independent mechanisms. J Biol Chem. 2005;280:24004-24010.

100. Wu JK, Kitajewski JK. A potential role for Notch signaling in the pathogenesis and regulation of hemangiomas. J Craniofac Surg. 2009;20:698-702.

101. Fre S, Pallavi SK, Huyghe M, et al. Notch and Wnt signals cooperatively control cell proliferation and tumorigenesis in the intestine. Proc Natl Acad Sci USA. 2009;106:6309-6314.

102. Reid P, Holen I. Pathophysiological roles of osteoprotegerin (OPG). Eur J Cell Biol. 2009;88:1-17.

103. Vishvanath A. The Molecular Pathogenesis of Haemangioma (Ph.D. Dissertation). Wellington, New Zealand: Victoria University of Wellington; 2008.

104. Vishvanath A, Itinteang T, Tan ST, Day DJ. Infantile haemangioma expresses TRAIL, TRAIL receptors, osteoprotegerin and RANKL. J Clin Path. (in press).

105. Moran CS, Cullen B, Campbell JH, Golledge J. Interaction between angiotensin II, osteoprotegerin, and peroxisome proliferator-activated receptor-gamma in abdominal aortic aneurysm. J Vasc Res. 2009;46:209-217.

106. Conway J. Hypotensive mechanisms of beta blockers. Eur Heart J. 1983;4:43-51.

107. Dillon MJ, Ryness JM. Plasma renin activity and aldosterone concentration in children. BMJ. 1975;4:316-319.

108. Lavoie JL, Sigmund CD. Minireview: Overview of the reninangiotensin system. An endocrine and paracrine system. Endocrinology 2003;144:2179-2183.

109. Silver RB, Reid AC, Mackins CJ, et al. Mast cells: A unique source of renin. Proc Natl Acad Sci USA. 2004;101:1360713612.

110. Weber MA. Vasopeptidase inhibitors. Lancet 2001;358: $1525-1532$. 
11.6. Manuscript: Treatment Of Infantile Hemangioma With Captopril 
Treatment of Infantile Hemangioma with Captopril

\begin{tabular}{|c|c|}
\hline Journal: & Pediatrics \\
\hline Manuscript ID: & $2011-2175$ \\
\hline Article Type: & Case Report \\
\hline $\begin{array}{r}\text { Date Submitted by the } \\
\text { Author: }\end{array}$ & 14-Aug-2011 \\
\hline Complete List of Authors: & $\begin{array}{l}\text { Tan, Swee; Hutt Hospital, Wellington Regional Plastic, Maxillofacial } \\
\text { \& Burns Unit } \\
\text { Itinteang, Tinte; Hutt Hospital, Wellington Regional Plastic, } \\
\text { Maxillofacial \& Burns Unit } \\
\text { Day, Darren; Victoria University of Wellington, School of Biological } \\
\text { Sciences } \\
\text { O'Donnell, Clare; Starship Hospital, Paediatric and Congenital } \\
\text { Cardiac Service } \\
\text { Mathy, Jon; Hutt Hospital, Wellington Regional Plastic, Maxillofacial } \\
\text { \& Burns Unit } \\
\text { Leadbitter, Philip; Hutt Hospital, Department of Paediatrics }\end{array}$ \\
\hline Keyword/Category: & Hemangioma, Angiogenesis, Therapies, Therapeutics \\
\hline
\end{tabular}

\section{SCHOLARONE"}

Manuscripts 


\section{Treatment of Infantile Hemangioma with Captopril}

${ }^{1-4}$ Swee T Tan, ONZM, MBBS FRACS, PhD, ${ }^{1-3}$ Tinte Itinteang, MBBS, ${ }^{1-3}$ Darren J Day, $\mathrm{PhD},{ }^{5} \mathrm{Clare} \mathrm{O}$ 'Donnell, MBChB, FRACP, ${ }^{1} \mathrm{~J}$ on A Mathy, MD, ${ }^{1,6}$ Philip Leadbitter, $\mathrm{MBChB}, \mathrm{FRACP}$

${ }^{1}$ Centre for the Study \& Treatment of Vascular Birthmarks, Wellington Regional Plastic, Maxillofacial \& Burns Unit, Hutt Hospital; ${ }^{2}$ Gillies McIndoe Research Institute; ${ }^{3}$ School of Biological Sciences, Victoria University of Wellington, Wellington; ${ }^{4}$ Wellington School of Medicine \& Health Sciences, University of Otago; ${ }^{5}$ Paediatric and Congenital Cardiac Service, Starship Hospital, Auckland; and ${ }^{6}$ Department of Paediatrics, Hutt Hospital, Wellington, New Zealand.

Short Title: Captopril for hemangioma

Key words: Infantile hemangioma, treatment, renin, angiotensin converting enzyme, inhibitor, captopril

Financial Disclosure: The authors do not have any financial interest with institutions, organizations, or companies that are mentioned in the manuscript or whose products or services are discussed.

Conflict of Interest: The authors do not have any conflict of interest 


\section{Corresponding Author and Request for Reprints:}

Professor Swee T Tan, MBBS FRACS PhD, Wellington Regional Plastic, Maxillofacial \& Burns Unit, Hutt Hospital, High Street, Private Bag 31-907, Lower Hutt, New Zealand.

Tel: +64 45872506 Fax: +64 45872510 Em: $\underline{\text { swee.tan@ huttvalleydhb.org.nz }}$

Parts of this work were presented at the Joint Annual Scientific Meeting of the American Society of Plastic Surgeons and the Canadian Society of Aesthetic Plastic Surgeons, Toronto, Canada, October $1-5,2010$; the $80^{\text {th }}$ Annual Scientific Congress of the Royal Australasian College of Surgeons, Adelaide, Australia, 2 - 6 May, 2011; the $16^{\text {th }}$ World Congress of the International Confederation for Plastic, Reconstructive and Aesthetic Surgery, Vancouver, Canada, May 21 - 27, 2011; and the Plastic Surgery Congress, Gold Coast, Australia, July 7 - 10, 2011.

Abbreviations: IH: infantile hemangioma; RAS: renin-angiotensin system; ACE: angiotensin converting enzyme; $\mathrm{ACEi}$ : angiotensin converting enzyme inhibitor; ATII: angiotensin II

This trial is registered ACTRN12611000523909 


\begin{abstract}
Background Our observation that $\mathrm{IH}$ is due to an aberrant proliferation and differentiation of a primitive mesoderm-derived hemogenic endothelium regulated by the renin-angiotensin system (RAS) has led us to propose angiotensin converting enzyme (ACE) as a potential therapeutic target.

Methods After initial screening investigations, infants with problematic IHs were admitted for initiation of captopril with a $0.1 \mathrm{mg} / \mathrm{kg}$ test dose orally, followed by dose escalation to $0.3 \mathrm{mg} / \mathrm{kg} /$ dose 8 -hourly over 24 hours. The dose increased up to $0.5 \mathrm{mg} / \mathrm{kg} /$ dose 8 -hourly one week later, if a noticeable involution had not already occurred. The response of $\mathrm{IH}$ to captopril was documented clinically and photographically before and after treatment and any side effect was recorded.

Results Two males and six females aged 5-22 (mean 12.9) weeks with problematic IH causing ulceration $(n=5)$, impending visual obstruction $(n=1)$, and/or significant tissue distortion $(n=8)$ were included. The offending lesions were located in nasal tip $(n=1)$, cervico-facial $(n=3)$, peri-orbital $(n=1)$ and perineal $(n=2)$ areas, and shoulder $(n=1)$. Transient mild renal impairment occurred in one subject which resolved spontaneously. No other complication was observed. A dose of captopril $1.5 \mathrm{mg} / \mathrm{kg} / \mathrm{day}$ led to accelerated involution of the IHs in seven subjects. IHs in one subject who required a slower dosage escalation underwent gradual involution. Continued involution of $\mathrm{IHs}$ was observed during the follow-up period of 2.5-9.5 (mean 6.9) months in all subjects. Conclusion The response of IH to an ACE inhibitor confirms a critical role for RAS in $\mathrm{IH}$ and represents a paradigm shift in the understanding and treatment of this enigmatic condition.
\end{abstract}




\section{Introduction}

Infantile hemangioma ( $\mathrm{IH})$ is the most common tumor of infancy affecting $10 \%$ of Caucasians. It is characterized by rapid proliferation during infancy followed by spontaneous slow involution over the next $5-10$ years $^{1-3}$.

In the absence of an effective treatment, the management of $\mathrm{IH}$ has been largely conservative. However, $10-15 \%$ of IHs require intervention during infancy because of the threat to life or function or tissue distortion or destruction ${ }^{1}$, with the mainstay treatment being pharmacologic therapy most of which had been discovered serendipitously ${ }^{1}$.

High dose corticosteroids have been the first-line therapy for problematic IHs for over four decades ${ }^{1}$. In $2008 \beta$-blockers, propranolol ${ }^{4}$ and acebutalol, ${ }^{5}$ were serendipitously discovered to cause accelerated involution of $\mathrm{IH}$, and led to a paradigm shift in the management of these tumors. $\beta$-blockers, particularly propranolol, have now replaced steroid therapy as the first-line treatment for proliferating $\mathrm{IH}$ worldwide. The rapid adoption of propranolol for this novel indication in the absence of proper clinical trials has justifiably attracted caution ${ }^{1,6}$, especially in light of reported cases of near fatal hypoglycaemia ${ }^{7,8}$. Most protocols have used the cardiovascular dose of $2-3 \mathrm{mg} / \mathrm{kg} / \mathrm{day}$. However, we have recently shown the effectiveness of low dose propranolol using a slow escalation and divided dosing regimen ${ }^{2,3}$ which goes some way to minimise the potential side effects.

We have also recently shown the expression of components of the reninangiotensin system (RAS) by the endothelium of proliferating $\mathrm{IH}^{14}$ and that $\mathrm{IH}$ consists of a hemogenic endothelium regulated by $\mathrm{RAS}^{14}$, potentially via the TRAIL-OPG apoptotic pathway ${ }^{15}$. 
We infer that the observed biologic effect of $\beta$-blockers is mediated via the modulation of the RAS and we proposed that angiotensin converting enzyme (ACE) and the angiotensin receptors as potential targets for the treatment of this condition ${ }^{1,14}$.

We present here the initial results of our observational clinical trial using captopril, an ACE inhibitor (ACEi), in the treatment of problematic proliferating $\mathrm{IH}$.

\section{Methods}

Infants with problematic $\mathrm{IH}$ were recruited into the captopril trial according to a New Zealand National Ethics Committee approved protocol. The exclusion criteria included neonates, cardiac outflow tract obstruction, renal impairment, diuretics administration or known allergy to ACEi. Following screening electrocardiogram, echocardiogram (to exclude cardiac outflow tract anomalies), and urea and electrolytes analysis (to exclude renal impairment), subjects were admitted for initiation of captopril. A test dose of $0.1 \mathrm{mg} / \mathrm{kg}$ was administered orally with pulse rate and blood pressure monitored at 0.5 , 1 , and 2 hours. If the test dose was tolerated, captopril was started at $0.15 \mathrm{mg} / \mathrm{kg} / \mathrm{dose} 8$ hourly. Pulse rate and blood pressure were monitored 4-hourly and doses witheld if hypotension was documented. After 24 hours, the dose was increased to $0.3 \mathrm{mg} / \mathrm{kg} / \mathrm{dose}$ 8-hourly and then a further 24 hours of observation. Urea and electrolytes analysis was performed at 48 hours, prior to discharge. The subjects were reviewed in clinic one week later when the dose was increased to $0.5 \mathrm{mg} / \mathrm{kg} /$ dose 8 -hourly if a noticeable involution had not already occurred, and a blood pressure check done following administration of the increased dose. For safety reasons we had limited the maximal dosage to $1.5 \mathrm{mg} / \mathrm{kg} /$ day. Escalation of the dosage was ceased at any stage if visible shrinkage of the IH was observed or if there was any adverse effect noted. 
Thereafter the subjects were reviewed again at one month and then at 3-monthly intervals. A full clinical examination was performed during each visit including growth chart measurements, cardiovascular status and any side effect was recorded. The response of the $\mathrm{IH}$ to captopril was documented clinically and by serial photographs before treatment and at every visit. Urea and electrolytes analysis was performed at one week, one month, three months, and one year or at any other time if clinically indicated.

\section{Results}

Two male and six female subjects aged 5 - 22 (mean 12.9) weeks referred to the Centre for the Study \& Treatment of Vascular Birthmarks with problematic IHs causing ulceration, bleeding and pain $(n=5)$ or impending visual obstruction $(n=1)$, and/or significant tissue distortion $(n=8)$ were included in the study (Table 1). None of the subjects were excluded from the study. Three subjects were born 2, 3, and 4 weeks prematurely. The average number of lesions was 3 (range 1 - 20) per patient. The offending lesion(s) was located in the nasal tip $(n=1)$, cervico-facial $(n=3)$, peri-orbital $(n=1)$, perineal $(n=2)$ areas, and shoulder $(n=1)$. One patient developed transient mild elevation of creatinine within the first week of captopril treatment which responded to a small dose reduction. The subject subsequently was able to reach a dosage of $1.5 \mathrm{mg} / \mathrm{kg} / \mathrm{day}$ with normal creatinine. Interestingly, the delayed dose escalation in this subject was associated with a slower rate of involution of the lesions.

The dose of captopril was increased to $0.5 \mathrm{mg} / \mathrm{kg} / \mathrm{dose} 8$-hourly in all subjects. Accelerated involution was observed (Figures 1 and 2) in seven subjects (27 lesions) with healing of the ulcerated lesions within 2 - 3 weeks in all five subjects (Figures 3 and 4$)$. In the remaining subject (2 lesions) the IHs underwent gradual involution with the lesions becoming softer, paler and smaller. In all subjects there was on-going 
involution of the lesions during the follow-up period of 2.5 - 9.5 (mean 6.9) months. Except for the transient mild elevation of creatinine during dose escalation in one subject, no hypotension or other adverse side effect was observed.

\section{Discussion}

The biology of $\mathrm{IH}$ has remained enigmatic. $\mathrm{IH}$ has been considered a tumor of the microvasculature, however, our recent data shows that it is a developmental anomaly involving aberrant proliferation and differentiation of a hemogenic endothelium ${ }^{9}$ derived from a primitive mesoderm with a neural crest phenotype ${ }^{10}$. This is further substantiated by the observation that cells from proliferating $\mathrm{IH}$ possess the ability to undergo erythropoietic ${ }^{11}$ and mesenchymal ${ }^{12}$ differentiation. The demonstration of embryonic stem cell associated proteins in proliferating $\mathrm{IH}$ suggests that this lesion contains cells with an early embryonic origin ${ }^{13}$. We have further provided evidence showing a placental chorionic villous mesenchymal core cell origin of $\mathrm{IH}^{16}$.

The poor understanding of the biology of $\mathrm{IH}$ has led to the on-going reliance on empirical treatments for this common condition ${ }^{1}$. High dose steroids have been the firstline therapy for problematic proliferating IH since it was serendipitously discovered in $1967^{17}$ despite their known side-effects and failure rates ${ }^{18}$. Vincristine had more recently emerged as an alternative to interferon for steroid-resistant IHs because of the adverse effects associated with interferon, particularly with regard to neurotoxiciy ${ }^{1}$. Since the serendipitous discovery that $\beta$-blockers can induce accelerated involution of $\mathrm{IH}$ by two independent French groups in $2008^{4,5}$, treatment with $\beta$-blockers is now the preferred first-line therapy for problematic IHs in spite of the potential adverse effects including hypoglycemia and the lack of adequate clinical trials. 
While $\beta$-blockers have a long and good safety record for the treatment of young children with hypertension and other cardiovascular conditions ${ }^{19}$, this may not be simply extrapolated to infants (some of whom are born prematurely) and young children with $\mathrm{IH}$, who are otherwise normo-tensive ${ }^{1}$. Caution has justifiably been raised regarding the casual use of this group of drugs for this novel indication in an otherwise normo-tensive population ${ }^{1,6}$. The potential adverse effects of $\beta$-blockers include bradycardia, hypotension, bronchospasm, hyperkalemia, and hypoglycemia ${ }^{1,7,8,20}$ with recent reports highlighting symptomatic and potentially fatal hypoglycemia in this patient cohort ${ }^{7,8}$. This underscores the need for large-scale clinical trials to delineate the optimal treatment protocol and safety issues with the use of $\beta$-blockers for this new indication, but also the development of better targeted and safer therapy.

The observed biologic effect of $\beta$-blockers has led to the speculation of the cellular and molecular pathways involved. A number of $\beta$-blockers used for the treatment of proliferating IH including systemic propranolol ${ }^{4}$, a non-selective $\beta$-blocker; acebutalol $^{5}$, a predominantly $\beta_{1}$-adrenoreceptor blocker; and topical timolol ${ }^{25,26}$, a nonselective $\beta$-blocker, suggests that the mechanism of action on $\mathrm{IH}$ relates to the class of drug rather than the adrenergic effect.

There is increasing evidence that $\mathrm{IH}$ is a disorder of aberrant proliferation and differentiation of primitive mesoderm-derived neural crest phenotypic cells ${ }^{10}$. This primitive phenotype that gives rise to a hemogenic endothelium intermediate ${ }^{9}$ has the ability to undergo primitive erythropoiesis ${ }^{11}$ and terminal mesenchymal ${ }^{12}$ differentiation.

We have also shown that the cells derived from proliferating $\mathrm{IH}$ form blasts in response to the administration of angiotensin II (ATII) ${ }^{14}$. This is further supported by the observation of a higher incidence of $\mathrm{IH}$ in Caucasians, female and premature infants corresponding with higher levels of renin within these groups ${ }^{1,14}$. We proposed that the 
high levels of renin in these patient groups, coupled with the local expression of ACE within proliferating IH, sustain a high local concentration of ATII. ${ }^{14}$ The binding of ATII to angiotensin receptor- 2 within proliferating IH thus drives proliferation of the hemogenic endothelium and downstream endothelial progenitor cells and endothelial cells ${ }^{14}$.

The expression of the components of RAS by the endothelium of proliferating $\mathrm{IH}$ and the observed in vitro effects of ATII on IH led us to conduct a clinical trial using captopril, an ACEi. The observed clinical effect of captopril on proliferating $\mathrm{IH}$ that we report here further supports the hypothesis that $\beta$-blockers do not exert their effects through the adrenergic system directly, but rather through their effect on RAS $^{1,14}$. Confirmation of the role of RAS in regulating $\mathrm{IH}$ progression is demonstrated by the evidence that inhibition of either of the two enzymes, renin or ACE, necessary for ATII production can lead to accelerated involution of $\mathrm{IH}$. Consistent with our hypothesis that ATII plays a vital role ${ }^{14}$ are reports demonstrating decreased renin levels in subjects taking $\beta$-blockers ${ }^{21}$. Conversely increased renin levels have been reported in subjects taking $\mathrm{ACEi}^{21}$, suggesting that blocking renin in addition to ACE may lead to a great effect on the involution of $\mathrm{IH}$ than already demonstrated in this study. Alternatively there may be further benefit to targeting downstream product of ATII, angiotensin receptor- 2 shown to be expressed by the endothelium of proliferating $\mathrm{IH}^{14}$. Efforts to examine this are currently underway by our group.

The use of captopril for cardiac failure in infants is well documented with a maximum dose of $2 \mathrm{mg} / \mathrm{kg}$ thrice daily $\mathrm{y}^{22}$, with caution against renal insufficiency, oliguria and hypotension, although these side effects are usually reversible upon dose reduction ${ }^{23-25}$. In our pilot trial the maximum dose of captopril administered was at the lower end of the therapeutic cardiovascular dosing yet we were able to demonstrate 
accelerated involution of $\mathrm{IH}$. This indicates the possibility of further dose escalation for improved efficacy. For safety reasons we elected not to escalate the dose of captopril beyond this upper dose limit of $1.5 \mathrm{mg} / \mathrm{kg} /$ day and we strongly recommend caution with the use of this medication in this population pending further clinical trials.

The concept of treating a condition such as $\mathrm{IH}$, considered to be a tumor of the microvasculature, with traditional anti-hypertensives to modulate the RAS, reveals a novel concept in the critical control of the RAS on IH stem/progenitor cell proliferation and novel mechanisms on the action of these relatively commonly used medications. Further trials of ACEi for this new indication will define the precise role of this group of drugs in the management of this challenging condition. 


\section{Acknowledgments}

We thank Dr Andrew Aitken FRACP, consultant cardiologist at Wellington Regional Hospital for his advice on this study and the timely reports of the screening echocardiograms. This work is supported by grants from the Gillies McIndoe Foundation and the Wellington Regional Plastic Surgery Unit Research \& Education Trust.

\section{Authors' Contributions}

STT, PL, JAM, CO'D, TI and DJD were involved in the design of the study

TI performed the literature search

STT and PL carried out data collection, analysis, and interpretation

STT provided and formatted the figures

STT and TI carried out manuscript preparation

PL, JAM, CO'D and DJD commented on the manuscript 


\section{References}

1. Itinteang T, Withers AHJ, Leadbitter P, Day DJ, Tan ST. Pharmacologic therapies for infantile hemangioma - is there a rational basis? Plast Reconstr Surg. 2011; DOI: 10.1097/PRS.0b013e31821b63a0.

2. Tan ST, Itinteang T, Leadbitter P. Low dose propranolol for multiple infantile hepatic and cutaneous hemangiomas with deranged liver function. Pediatrics 2011; 127: e772-776.

3. Tan ST, Itinteang T, Leadbitter P. Low-dose propranolol for infantile haemangioma. J Plast Reconstr Aesth Surg. 2010; 64 : 292-299.

4. Leaute-Labreze C, Dumas de la Roque, Hubiche T, Boralevi F, Thambo JB, Taieb A. Propranolol for severe hemangiomas of infancy. $N$ Engl J Med. 2008; 358: 2649-2651.

5. Bigorre M, Van Kien AK, Valette H. Beta-blocking agent for treatment of infantile hemangioma. Plast Reconstr Surg. 2009; 123 : e195-196.

6. Siegfried EC, Keenan WJ, Al-Jureidini S. More on propranolol for hemangiomas of infancy. N Engl J Med. 2008; $70: 2846-2847$.

7. Boniface E, Acquafredda A, Milano A, Montagna O, Laforgia N. Severe hypoglycemia during successful treatment of diffuse hemangiomatosis with propranolol. Pediatr Dermatol. 2010; 27 : 195-196.

8. Holland KE, Freiden IJ, Frommelt PC, Mancini AJ, Wyatt D, Drolet BA. Hypoglycemia in children taking propranolol for the treatment of infantile hemangioma. Arch Dermatol. 2010; 146 : 775-778.

9. Itinteang T, Tan ST, Brasch H, Day DJ. Haemogenic endothelium in infantile haemangioma. J Clin Pathol. 2010; 63 : 982-986.

10. Itinteang T, Tan ST, Brasch H, Day DJ. Primitive mesodermal cells with a neural crest phenotype predominate proliferating infantile haemangioma. $J$ Clin Pathol. 2010; 63 : 771-776.

11. Itinteang $\mathrm{T}$, Tan ST, Brasch HD, Vishvanath A, Day DJ. Primitive erythropoiesis in infantile haemangioma. Brit J Dermatol. 2011; 164 : 109710100.

12. Itinteang T, Vishvanath A, Day DJ, Tan ST. Mesenchymal stem cells in infantile haemangioma. J Clin Pathol. 2011; 64 : 232-236.

13. Itinteang $\mathrm{T}$, Tan ST, Brasch HD, Steel R, Best HA, Day DJ. Infantile haemangioma expresses embryonic stem cell markers. Brit J Dermatol, 2011; (under revision).

14. Itinteang T, Brasch HD, Tan ST, Day DJ. Expression of components of the renin-angiotensin system in proliferating infantile haemangioma may account for the propranolol-induced accelerated involution. J Plast Reconstr Aesthet Surg. 2011; $64: 759-765$.

15. Vishvanath A, Itinteang T, Tan ST, Day DJ. Infantile haemangioma expresses TRAIL, TRAIL receptors, osteoprotegerin and RANKL. Histopathology. 2011; (in press). 
16. Itinteang T, Tan ST, Guthrie S, Tan CES, McIntyre BC, Brasch HD, Day DJ. A placental chorionic villous mesenchymal core cellular origin for infantile haemangioma. J Clin Pathol. 2011; (in press).

17. Zarem HA, Edgerton MT. Induced resolution of cavernous hemangiomas following prednisolone therapy. Plast Reconstr Surg. 1967; 39 : 76-83.

18. Tan BH, Leadbitter P, Auburn N, Tan ST. Steroid therapy for problematic proliferating haemangioma. NZ Med J. 2011; 124 : 1329-1338.

19. Artman M, Grayson M, Boerth RC. Propranolol in children: safety-toxicity. Pediatrics. 1982; $70: 30-31$.

20. Pavlakovic H, Kietz S, Lauerer P, Zutt M, Lakomek M. Hyperkalemia complicating propranolol treatment of an infantile hemangioma. Pediatrics 2010; 6: e1589-1593.

21. Holmer SR, Hengstenberg C, Mayer B, Engel S, Lowel H, Riegger GAJ, Schunkert H. Marked suppression of renin levels by beta-receptor blocker in patients treated with standard heart failure therapy: a potential mechanism of benefit from beta-blockade. J Internal Medicine. 2001; 249: 167-172.

22. Sinaiko AR, Mirkin BL, Hendrick DA, Green TP, O'Dea RF. Antihypertensive effect and elimination kinetics of captopril in hypertensive children with renal disease. J Pediatr. 1983: 799-805.

23. Scammell AM, Arnold R, Wilkinson JL. Captopril in treatment of infant heart failure: a preliminary report. Inter J Cardiology. 1987; $16: 295-301$.

24. Shaw NJ, Wilson N, Dickinson DF. Captopril in heart failure secondary to a left to right shunt. Arch Dis Child. 1988; $63: 360-363$.

25. Gantenbein MH, Bauersfeld U, Baenziger O, Frey B, Neuhaus T, Sennhauser F, Bernet V. Side effects of angiotensin converting enzyme inhibitor (captopril) in newborns and young infants. J Perinat Med. 2008; 36 : 448-452.

26. Pope E, Chakkittakandiyil. Topical timolol gel for infantile hemangiomas: a pilot study. Arch Dermatol. 2010; $146: 564-5$.

27. Guo S, Ni N. Topical treatment for capillary hemangioma of the eyelid using $\beta$ blocker solution. Arch Opthalmol. 2010; 128 : 255-256. 


\section{Figure Legends}

Figure 1. A 22-week old girl with a $7 \times 10 \mathrm{~cm}$ proliferating $\mathrm{IH}$ in the right cervicofacial area causing significant tissue distortion before (A \& B), three weeks (C), and 6 months (D \& E) after administration of captopril at $1.5 \mathrm{mg} / \mathrm{kg} /$ day resulting in accelerated involution.

Figure 2. A 20-week old girl with a nasal tip proliferating IH causing a Cyrano de Bergerac deformity. Before (A \& B) and 7 months after (C \& D) captopril treatment at $1.5 \mathrm{mg} / \mathrm{kg} /$ day.

Figure 3. A 5-week old girl with a $4 \times 5 \mathrm{~cm}$ painful deeply penetrating ulcerated proliferating $\mathrm{IH}$ on the left groin. Before (A), 2 weeks (B), and 5.5 months (C) after captopril treatment at $1.5 \mathrm{mg} / \mathrm{kg} / \mathrm{day}$.

Figure 4. A 13-week old girl born 4 weeks prematurely with a $4 \times 5 \mathrm{~cm}$ ulcerated proliferating $\mathrm{IH}$ on the left perianal area and 20 other lesions on the limbs, trunk and upper lip measuring 0.5 - $4 \mathrm{~cm}$. Before (A), 3 weeks (B), 6 weeks (C), and 4 months (D) after captopril treatment at $1.5 \mathrm{mg} / \mathrm{kg} /$ day. 
Page 15 of 19

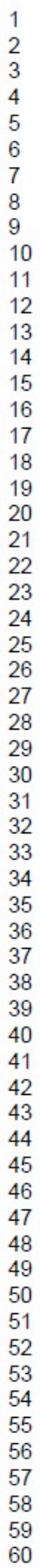

Confidential - Not for Circulation

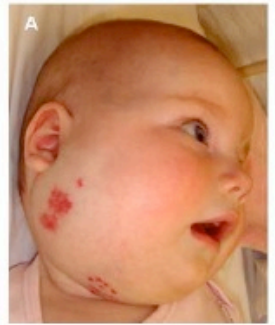

Figure 1

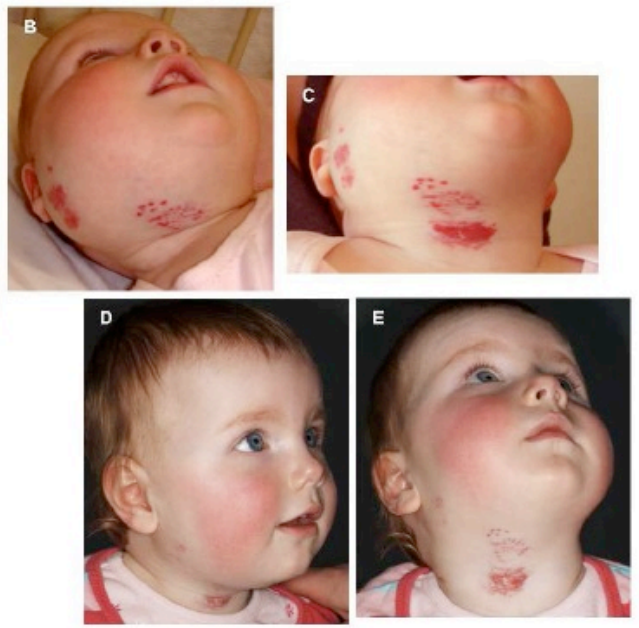

Fiqure 1. A 22-week old girl with a $7 \times 10 \mathrm{~cm}$ proliferating IH in the right cervico-facial area causing significant tissue distortion before (A \& B), three weeks (C), and 6 months (D \& E) after administration of captopril at $1.5 \mathrm{mg} / \mathrm{kg} /$ day resulting in accelerated involution. $285 \times 190 \mathrm{~mm}(96 \times 96 \mathrm{DPI})$ 

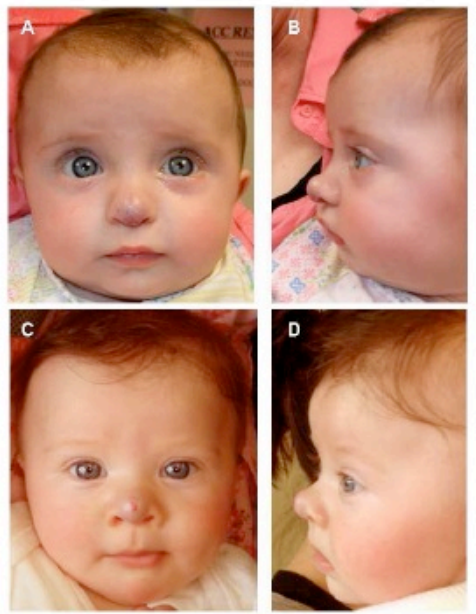

Figure 2

Fiqure 2. A 20-week old girl with a nasal tip proliferating IH causing a Cyrano de Bergerac deformity. Before (A\&B) and 7 months after (C \& D) captopril treatment at $1.5 \mathrm{mg} / \mathrm{kg} / \mathrm{day}$. $285 \times 190 \mathrm{~mm}(96 \times 96 \mathrm{DPI})$ 

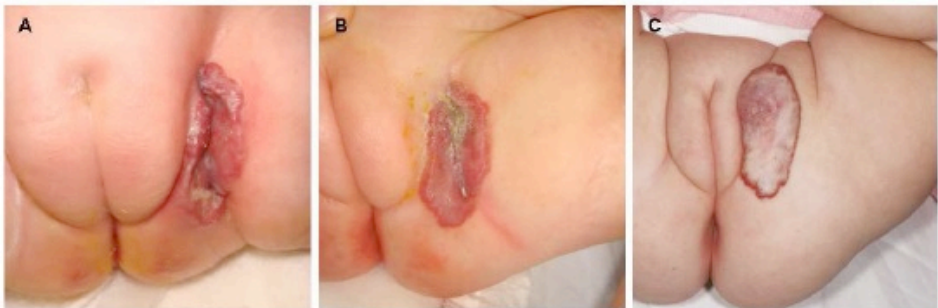

Figure 3

Figure 3. A 5 -week old girl with a $4 \times 5 \mathrm{~cm}$ painful deeply penetrating ulcerated proliferating IH on the left groin. Before (A), 2 weeks (B), and 5.5 months (C) after captopril treatment at $1.5 \mathrm{mg} / \mathrm{kq} / \mathrm{day}$.

$285 \times 190 \mathrm{~mm}(96 \times 96 \mathrm{DPI})$

The American Academy of Pediatrics, 141 Northwest Point Blvd., Elk Grove Village, IL 60007 


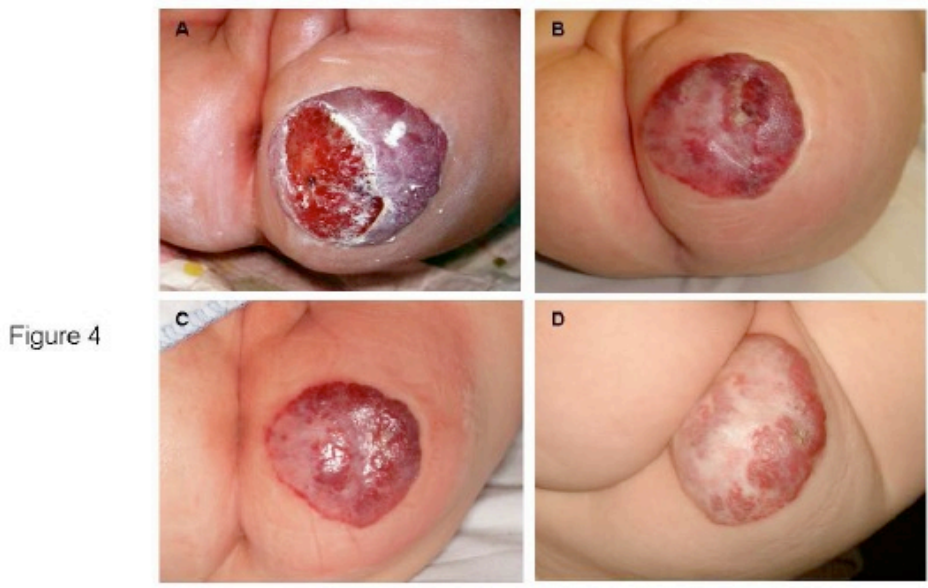

Figure 4. A 13-week old girl born 4 weeks prematurely with a $4 \times 5 \mathrm{~cm}$ ulcerated proliferating IH on the left perianal area and 20 other lesions on the limbs, trunk and upper lip measuring $0.5-4 \mathrm{~cm}$. Before (A), 3 weeks (B), 6 weeks (C), and 4 months (D) after captopril treatment at $1.5 \mathrm{mg} / \mathrm{kg} / \mathrm{day}$. $285 \times 190 \mathrm{~mm}(96 \times 96 \mathrm{DPI})$ 
Table 1 Patient Demographics and Response of Infantile Hemangioma to Captopril

\begin{tabular}{|c|c|c|c|c|c|c|c|c|c|c|}
\hline \multirow[t]{2}{*}{ Patient } & \multirow[t]{2}{*}{ Age } & \multirow[t]{2}{*}{ Sex } & \multirow{2}{*}{$\begin{array}{l}\text { Offending } \\
\text { Lesion }\end{array}$} & \multirow[t]{2}{*}{ Size $(\mathrm{cm})$} & \multirow[t]{2}{*}{ Symptoms } & \multicolumn{2}{|c|}{ Other Lesions } & \multirow[t]{2}{*}{ Response } & \multirow[t]{2}{*}{ Complications } & \multirow{2}{*}{$\begin{array}{c}\text { Follow up } \\
\text { (months) }\end{array}$} \\
\hline & & & & & & & Size $(\mathrm{cm})$ & & & \\
\hline MQ & $13 \mathrm{~W}$ & F & Perianal & $4 \times 5$ & $\begin{array}{c}\text { Ulceration* } \\
\text { Tissue distortion }\end{array}$ & $\begin{array}{l}20 \text { lesions limbs, } \\
\text { trunk \& lip }\end{array}$ & $0.5-4$ & $\begin{array}{l}\text { Accelerated } \\
\text { involution }\end{array}$ & $\begin{array}{c}\text { Transient mild } \\
\text { elevation of creatinine }\end{array}$ & 9.5 \\
\hline JH & $22 \mathrm{~W}$ & F & Parotid/neck & $7 \times 10$ & Tissue distortion & & - & $\begin{array}{l}\text { Accelerated } \\
\text { involution }\end{array}$ & & 7.5 \\
\hline $\mathrm{MMcF}$ & $20 \mathrm{~W}$ & F & Nose & $1.5 \times 1.5$ & $\begin{array}{c}\text { Cyrano de Bergerac } \\
\text { deformity }\end{array}$ & & - & $\begin{array}{l}\text { Accelerated } \\
\text { involution }\end{array}$ & & 7 \\
\hline $\mathrm{CH}$ & $5 \mathrm{~W}$ & F & Groin & $5 \times 4$ & Ulceration* & & - & $\begin{array}{l}\text { Accelerated } \\
\text { involution }\end{array}$ & & 9 \\
\hline $\mathrm{JK}$ & $13 \mathrm{~W}$ & M & Shoulder & $3 \times 2.2 .5$ & Ulceration* & Cheek & $3 \times 4$ & $\begin{array}{l}\text { Accelerated } \\
\text { involution }\end{array}$ & & 8 \\
\hline $\mathrm{MMaC}$ & $5 \mathrm{~W}$ & F & Lower eyelid & $3 \times 2$ & $\begin{array}{l}\text { Visual obstruction } \\
\text { Tissue distortion } \\
\end{array}$ & Hand & $3 \times 3$ & $\begin{array}{l}\begin{array}{l}\text { Gradual } \\
\text { involution }\end{array} \\
\end{array}$ & & 8 \\
\hline RS & $16 \mathrm{~W}$ & F & $\begin{array}{c}\text { Lower face/ } \\
\text { lip }\end{array}$ & $8 \times 7$ & $\begin{array}{c}\text { Ulceration* } \\
\text { Tissue distortion }\end{array}$ & & - & $\begin{array}{l}\text { Accelerated } \\
\text { involution }\end{array}$ & & 4 \\
\hline OW & $10 \mathrm{~W}$ & M & Retroauricular & $4 \times 5$ & $\begin{array}{c}\text { Ulceration* } \\
\text { Distortion of ear }\end{array}$ & Glabella & $2 \times 2$ & $\begin{array}{l}\text { Accelerated } \\
\text { involution }\end{array}$ & & 2.5 \\
\hline
\end{tabular}

*with bleeding and pain, fully healed within 2-3 weeks; M: male; F: female; W: weeks 


\section{Chapter 12: Conclusion}

$\mathrm{IH}$, the most common tumour of infancy, affects an average of $10 \%$ of infants with a predilection for Caucasian, female and premature infants ${ }^{4,5} . \mathrm{IH}$ undergoes an initial proliferation within the first year of life, followed by spontaneous involution that may take up to ten years ${ }^{5}$; the proliferating phase is characterised by aggressive angiogenesis whereas the involuted phase by fibrofatty deposition.

\subsection{Key Findings}

\subsubsection{Renin-Angiotensin System in Infantile Haemangioma}

The work in this thesis has shown a role for the renin-angiotensin system (RAS) in $\mathrm{IH}$, in the biology of $\mathrm{IH}$ and accounts for the observed propranololinduced accelerated regression of $\mathrm{IH}$. The observed effect of propranolol and the observation that renin levels are higher in Caucasian, female and premature infants who have an increased incidence of $\mathrm{IH}$, led me to investigate the expression of components of the RAS in IH. This work shows that propranolol exerts its therapeutic effects via inhibition of renin activity, rather than through beta adrenergic receptors as proposed by others ${ }^{25,98}$.

The role of renin in the aetio-pathology of $\mathrm{IH}$ was supported by previous observations showing very high levels of renin in infants compared with adults and increased levels of renin in patients with a higher risk of developing $\mathrm{IH}$, Caucasian (compared with back), female (compared with male) and premature (compared with term) infants. The identification of key 
components of the RAS, ACE, ATII and ATR2 and in vitro experiments demonstrating ATII-induced IH blast proliferation, confirm a key role for the RAS in the biology of $I H^{90}$. The novel discovery of the RAS pathway in the biology of $\mathrm{IH}$ identifies a potential role for ACEi as a potential therapy for $\mathrm{IH}$. A clinical trial using captopril, an ACEi, induced accelerated involution as predicted by our model and unequivocally proved the role of the RAS in IH progression. This concept has revolutionised the understanding of this enigmatic condition. However our preliminary results on the use of ACEi, while sucessful were less dramatic compared with propranolol. I hypothesised that the levels of ATII available to $\mathrm{IH}$, might involve a balance and/or combination of endocrine and paracrine/autocrine systems. I have recently (unpublished results) demonstrated the presence of local RAS/non-RAS pathways leading to the local production of the vasoactive peptide ATII in IH. This observation suggests that targeting the peptides upstream of ATII will possibly allow for the development of more effective treatment. Such treatment approach would negate the upstream accumilation of ATI resulting from treatment with ACEi which may be subsequently acted upon by other locally expressed proteases to generate vasoactive peptides other than ATII.

\subsubsection{Osteoprotegerin in Haemangioma}

The novel identification of OPG in IH suggests a potential role for OPG in the inhibition of apoptosis via the TRAIL receptors in proliferating $\mathrm{IH}$. The identification of abundant expression of OPG on immature microvessels but not the mature vessels suggest an important role for the anti-apoptotic TRAIL 
pathway during proliferation ${ }^{11}$. It is intriguing that ATII has also been demonstrated to promote secretion of $\mathrm{OPG}^{99}$. This further supports a critical role of the RAS, both in inducing proliferation and also in the maintenance of anti-apoptosis during the proliferaion of $\mathrm{IH}$.

Other reported functions of OPG involve its role in the stress response, possiblity due to inflammation, which is also a trigger for the formation of new vessels. It is my opinion that the restricted expression of OPG on the immature $\mathrm{IH}$ endothelium supports a role in an autocrine/paracrine response on anti-apoptosis.

\subsubsection{Haemogenic Endothelium and Primitive Erythropoiesis in} Infantile Haemangioma

IH has been proposed to be derived from circulating EPCs, based on the identification of increased levels of circulating EPCs in the peripheral blood of the affected patients. Exploration of this idea led us to discover not only the expression of EPC-like markers on the endothelium of proliferating $\mathrm{IH}$, but also the expression of more primitive haematopoietic markers, ACE, Tal$1 / \mathrm{SCL}$ and Gata2, denoting a haemogenic endothelium ${ }^{100}$. In further support of our suggestion that $\mathrm{IH}$ is derived from a haemogenic endothelium was the demonstration of expression of primitive erythropoietic associated proteins EPO-R and $\mathrm{Hb} \zeta$ as described in Chapter $5^{101}$. The proof that $\mathrm{IH}$ is a haemogenic endothelium is proven by the functional capacity of proliferating IH explant derived cells to form erythrocytes in vitro. These findings highlight 
$\mathrm{IH}$ not only as a potential extramedullary source of erythropoiesis, but also as a novel model for studying human primitive haematopoiesis.

\subsubsection{Primitive Mesoderm with a Neural Crest Stem cell phenotype in Haemangioma}

The process of primitive haematopoiesis and development of $\mathrm{IH}$ involve the identification of primitive mesoderm, crucial for forming downstream cells capable of primitive haematopoiesis. These primitive mesodermal cells are characterised by the expression of the transcription factor brachyury on the endothelial layer of proliferating $\mathrm{IH}$. These cells also express the neural crest stem cell associated markers, p75, Sox-9 \& Sox-10, highlighting these cells as potentially being the most primitive population in the proliferating capillaries. This unique neural crest phenotype may account for segmental $\mathrm{IH}$, some of which are associated with PHACES syndrome, which is discussed later in this chapter $^{102}$.

\subsubsection{Mesenchymal Stem Cells in Haemangioma}

The fibro-fatty deposition that predominates during involution highlights an observed change in the predominant cell type compared with those present during proliferation. This could either be explained by the invasion of MSC from adjacent niches that eventually differentiate into adipocytes as previously proposed $^{39}$, or by the identification of an innate MSC population within proliferating $\mathrm{IH}$ lesions. The data presented in Chapter 6 shows the expression of MSC proteins (vimentin and CD29) and mesenchymal 
differentiation inhibition protein (pref-1) by the cells of the endothelium (ref). These results are further supported by functional experiments highlighting the expression of the same proteins by cells emanating from proliferating $\mathrm{IH}$ explants, which show functional ability to differentiate into terminal mesenchymal cells, namely adipocytes and osteoblasts ${ }^{103}$. This confirms a mesenchymal differentiation potential of the haemogenic endothelium. My model does not exclude the possibility of invasion of MSCs from adjacent/other niches, however, my data confirm the presence of an innate MSC population within $\mathrm{IH}$.

\subsubsection{Embryonic Stem Cell expression in Infantile Haemangioma}

The demonstration of a neural crest stem cell phenotype in $\mathrm{IH}$, led us to investigate whether the $\mathrm{IH}$ lesions are derived from hESCs, from which during normal development, neural crest stem cells can be directly derived. Surprisingly hESC markers are identified, but their expression is identified on two different cell populations within proliferating $\mathrm{IH}$ lesions. One population express Oct-4, SSEA-4 and STAT-3 and is restricted to cells of the endothelium, whilst the other population that express nanog is located in the interstitium but none of the other ESC markers. This expression pattern within proliferating $\mathrm{IH}$ is intriguing, and at this stage we can only infer that either the Oct-4-positive cells within the endothelium represent the true cellular origin of $\mathrm{IH}$ which give rise to the 'more' downstream nanog-positive cell or that there are simply two different primitive populations of cells within proliferating lesions. However, more recent data has confirmed that the nanog-positive 
cells are also express mast cell markers (unpublished results), inferring a primitive myeloid phenotype that is most likely derived from the haemogenic endothelium.

\subsubsection{Placental Chorionic Villous Mesenchymal core cellular origin of Infantile Haemangioma}

Previous studies have reported on the similarities in the expression profile

between $\mathrm{IH}$ and the placenta, and suggest a placental origin for $\mathrm{IH}^{6,84,86}$ eventhough the IHC staining pattern is not completely consistent with a trophoblast origin ${ }^{87}$. These reported similarities between $\mathrm{IH}$ and placenta, and the ambiguities of previous investigations led us to investigate the expression of trophoblast-associated markers in $\mathrm{IH}$. Our data demonstrate the expression of the placental markers, hPL and hCG, but not CK7 or HLA-G. These data and the controls used, support the cellular origin of $\mathrm{IH}$ as being the mesenchymal core cells of the placental villi. This suggestion supports our earlier findings, as these placental mesenchymal core villous cells are derived from the primitive streak, that also expresses brachyury. ${ }^{84}$ This is quite intrugiung in that the primitive streak also gives rise to the primitive mesoderm of the embryonic yolk sac, the site of embryonic $\mathrm{Hb} \zeta$ production, as well as the site of de novo haemogenic endothelium formation. Taken as a whole, the data strongly supports a placental villous mesenchymal core cell origin for $\mathrm{IH}$. Embolisation of these cells early in the embryonic development may account for segmental lesions, and embolisation that occurs later in the embryonic development results in localised lesions. 
Our neural crest cell theory as giving rise to the PHACES has recently been quoted in a published article ${ }^{104}$ 'A new report emphasizes the possibility of an underlying neural crest abnormality contributing not only to the cardiovascular malformations but also to the hemangiomas [ltinteang et al.,2010]' confirming acknowledgement and support of our model by other groups.

\subsubsection{Summary of Key Findings}

The key finding of this thesis is that $\mathrm{IH}$ is developmental anomaly of stem cell proliferation and differentiation. These statements have been confirmed by the characterisation of a novel stem cell population in $\mathrm{IH}$ and the critical role of the RAS in both the natural biology of $\mathrm{IH}$ and the observed effect of novel treatments, such as propranolol and ACEi.

The physiologically higher levels of renin during infancy which taper off once a child reaches their first year of life and is normalised by the age of ten, and the high levels in Caucasian (compared with black), female (compared to male) and in premature (compared with termed) infants thus account for both the natural history of $\mathrm{IH}$ and the increased prevalence in female, Caucasian and premature infants.

Our data suggests that it is likely that placental mesenchymal core villous cells are the cell of origin of $\mathrm{IH}$. We infer that these stem cells are embolised to the foetus in utero, and account for the increased risk of $\mathrm{IH}$ 
following invasive in utero investigations, such as amniocentesis and chorionic villous sampling. These cells depending on the timing of embolisation may also account for the clinical presentations of $\mathrm{IH}$, whereby early embolisation leading to PHACES syndrome whereas 'late' embolisation accounting for discrete lesions.

Figure 12.1 highlights the key stages of stem cell differentiation during IH development. Physiologically high levels of circulating renin during infancy, favours formation of endothelial progenitor cells (EPCs) and haematopoiesis. As renin levels decrease during childhood resulting in reduced angiotensin II levels with subsequent reduced OPG. The net result of this is reduced EPCs, promotion of apoptosis and a release of the block on mesenchymal differentiation with the formation of the fibro-fatty tissue of involuted lesions. 


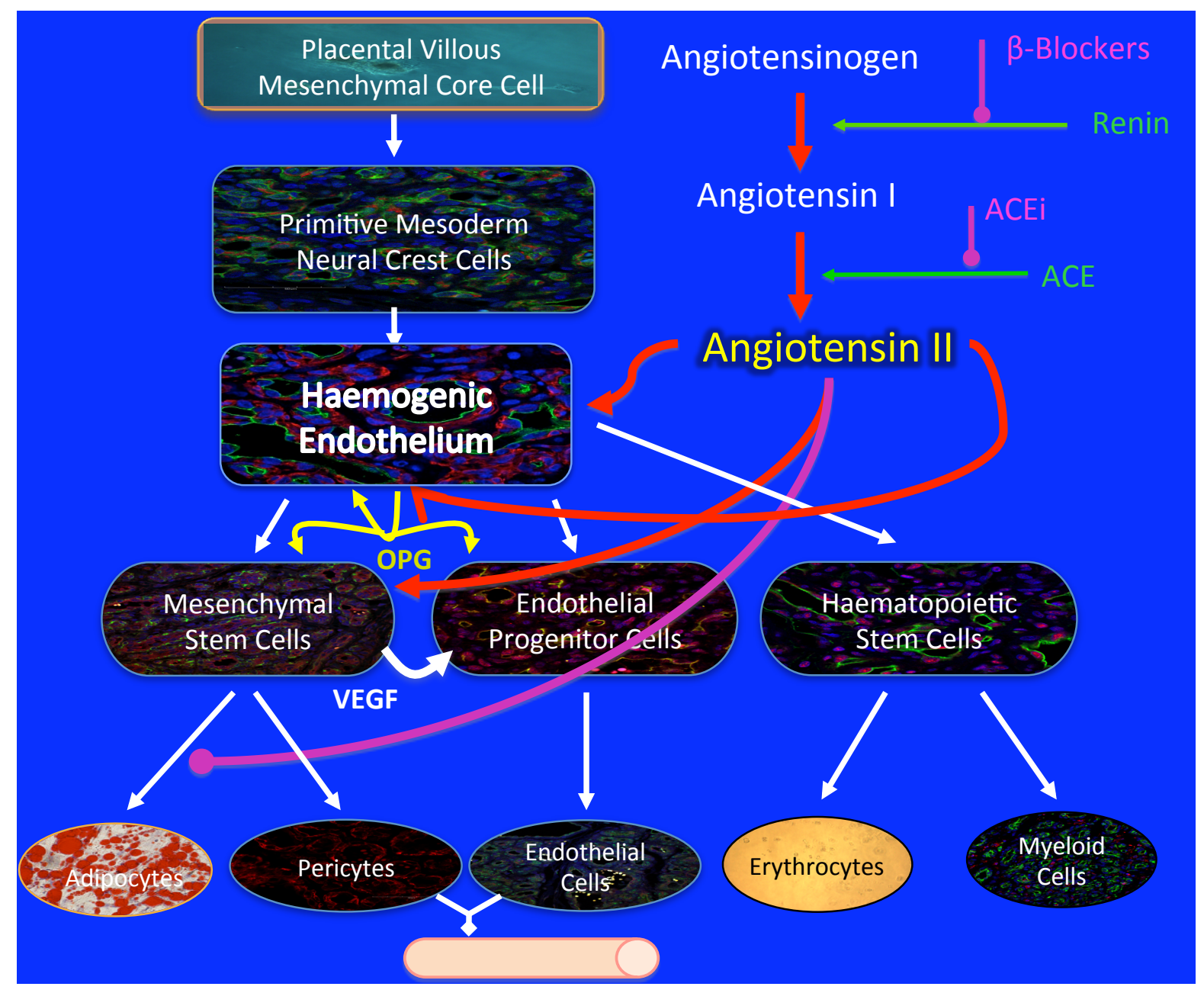

Figure 12.1. Our proposed model of the cellular lineages of $\mathrm{IH}$ and the role of the renin-angiotensin system and osteoprotegerin (OPG) in the development $\mathrm{IH}$.

\subsection{Future Directions in Haemangioma Research}

\subsubsection{Angiotensin II}

An understanding of the key peptides leading to the formation of ATII and its application to the developmental biology of $\mathrm{IH}$ highlights a future area in the regulatory steps in the biology of $\mathrm{IH}$ and the development of novel treatment 
strategies for $\mathrm{IH}$. This thesis has investigated the RAS pathway and its inferred role in IH development. However, other non- RAS pathways which also lead to the formation of the vasoactive ATII is now the topic of further investigation by our group. Preliminary data from our group have confirmed abundant expression of cathepsin B, an enzyme responsible for converting pro-renin to renin, and cyclo-oxgenase-2 (COX-2) within proliferating $\mathrm{IH}$ (Figure 12.2), constituting paracrine/autocrine non-RAS pathway within $\mathrm{IH}$. Figure 12.3, illustrates non-renin/ACE pathways leading to ATII formation. We have previous shown the expression of chymase by mast cells within $\mathrm{IH}$ (ref) and the obvious implications for their role in the biology in $\mathrm{IH}$. The applications of the role of ATII in IH and stem cell biology are still in their early stages and further research into their exact role and interplay with other vasoactive peptides, such as VEGF, will need to investigated.
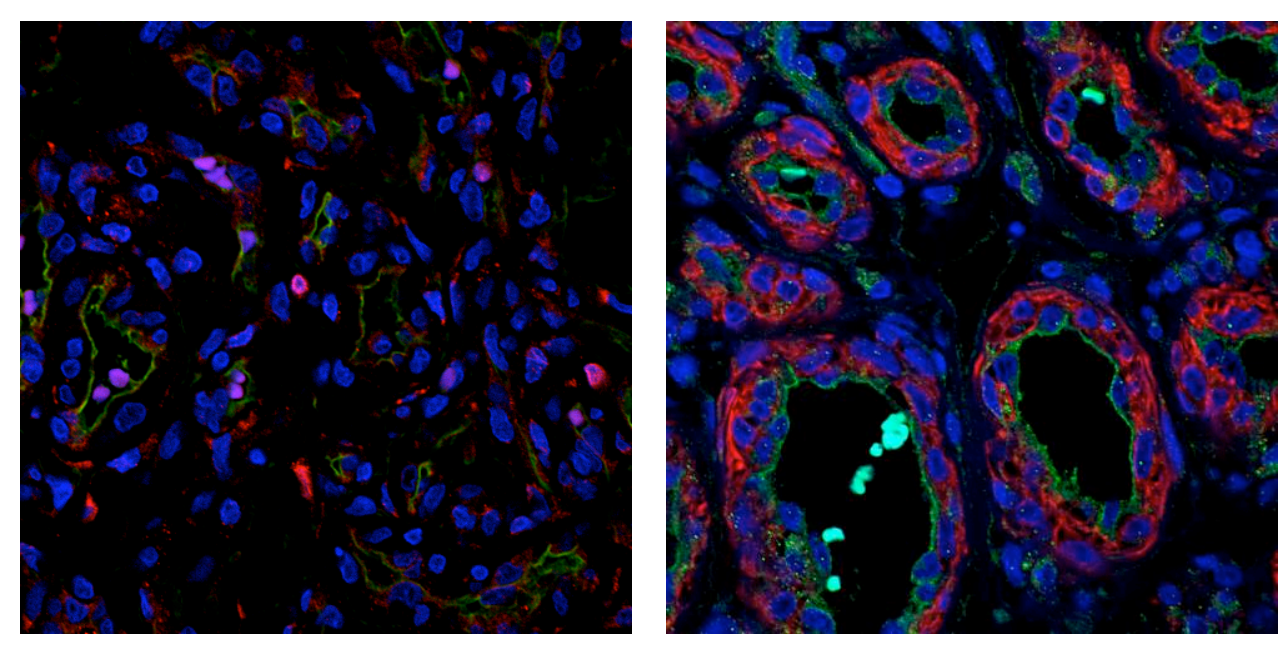

Figure 12.2. IHC of proliferating IH illustrating expression of COX-2 (red, left panel) and cathepsin B (green, right panel). 


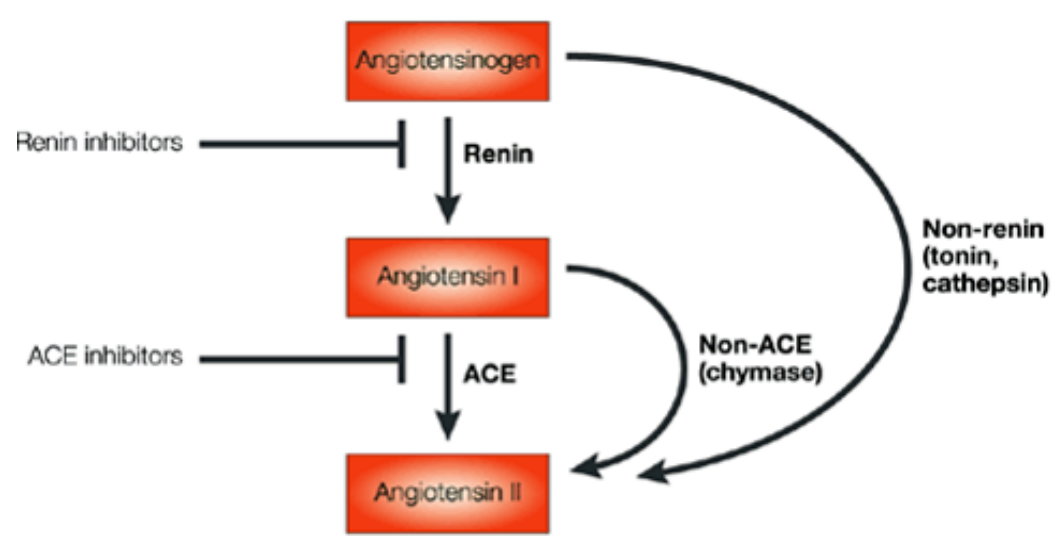

Nature Reviews | Drug Discovery

Figure 12.3. A schema illustrating both the RAS and the non-RAS pathways leading to ATII formation (Adapted from Zaman, et al ${ }^{105}$ ).

\subsubsection{Stem Cell Biology of Infantile Haemangioma}

The origin of $\mathrm{IH}$ has remained enigmatic. Identification of critical stages in stem cell differentiation in $\mathrm{IH}$ has enabled a better understanding of this condition. Future research in the development of animal models for $\mathrm{IH}$ will need to address not only the environmental cytokine milieu, but also an appreciation for the heterogeneity of the stem cell population in $\mathrm{IH}$. We now have data demonstrating that the nanog-positive cells in the interstitium of proliferating $\mathrm{IH}$ contained in Chapter 9, are the mast cells previously shown in $\mathrm{IH}^{5}$. Whether these mast cells originate from a primitive myeloid lineage derived from the haemogenic endothelium during primitive haematopoiesis or whether they are an independent primitive population within $\mathrm{IH}$ is a topic of our current research. These observations have highlighted some of the key 
stem cell components that make up $\mathrm{IH}$. It is therefore possible to infer that until such a model encompassing all facets of $\mathrm{IH}$ biology that the true in vivo model of IH maybe established, perhaps in an orthoptic model (Figure 12.4), thus enabling future advances in research into $\mathrm{IH}$ and a further appreciation of the complex aetio-pathology of this enigmatic condition.
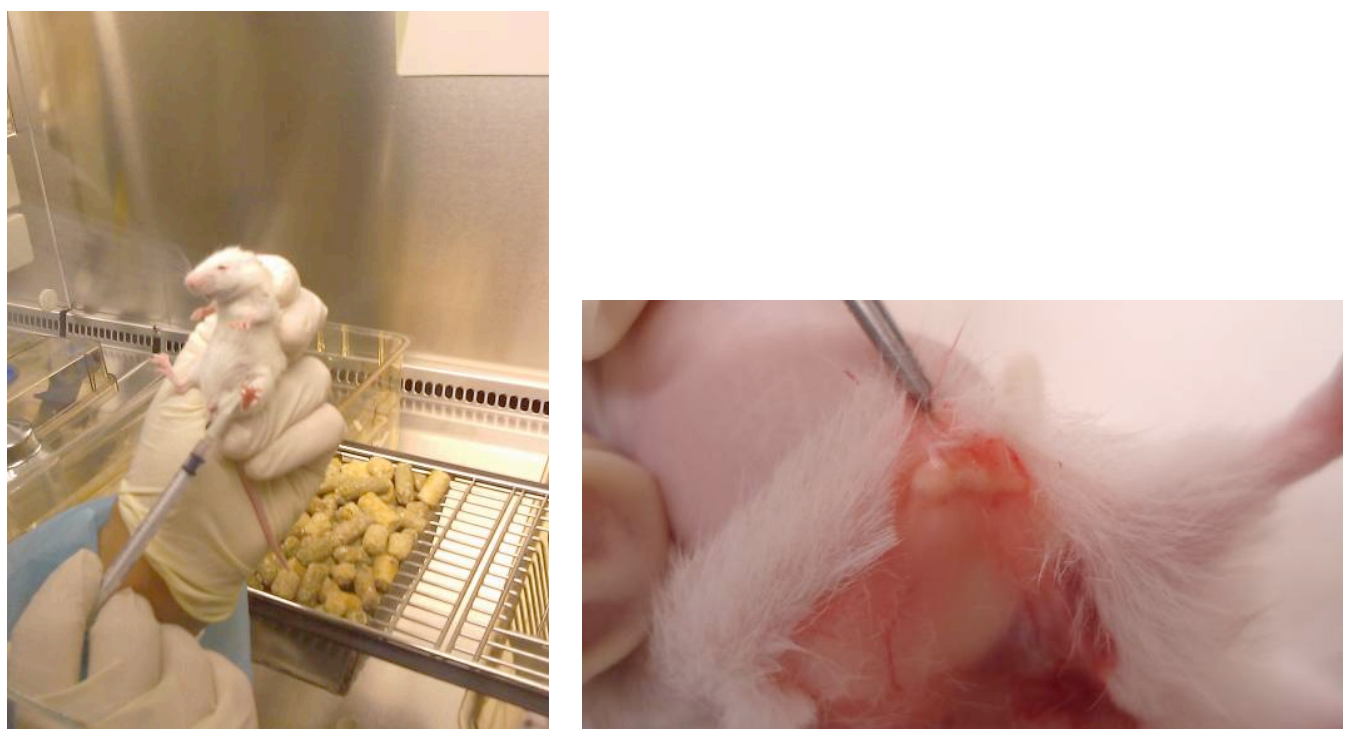

Figure 12.4. The development of an in vivo IH mouse model is dependant on the heterogenity of the stem cell population in $\mathrm{IH}$.

\subsubsection{Tissue Engineering}

The stem cell biology of $\mathrm{IH}$ and the ability of innate stem cells within $\mathrm{IH}$ to differentiate into a variety of downstream cellular elements such as adipocytes, osteoblasts and erythrocytes, further highlights the potential use of $\mathrm{IH}$ derived stem cells. These multipotent stem cells can be further used as 
models for developing a better understanding of the cytokines and the differentiation pathways that they control in maintaining differentiation. These pathways may potentially provide the launching platform for the development of directed stem cell differentiation and its myriad of applications in the field of tissue engineering (Figure 12.5).

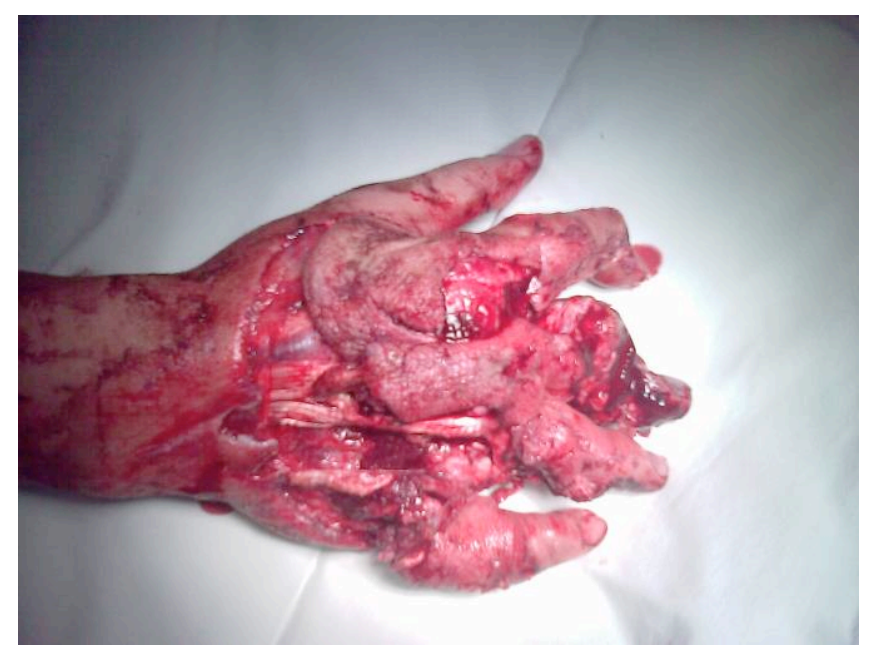

Figure 12.5. The clinical applications for the future use of tissue engineering will involve the management of mutilating injuries.

\subsubsection{Tumour Vasculogenesis}

There is accumulating evidence showing cancer stem cells in solid tumours are dependant upon the ability to establish their own blood supply for nutrient access, but which also facilitates metastasis ${ }^{106}$. The development of a tumour 
blood supply, tumour vasculogenesis, is dependant on the resident stem cell population with vascuologenic differentiation capacity. Our research in vasculogenesis in $\mathrm{IH}$ has highlighted the expression of key proteins in the resident stem cell populations (Figure 12.6). Our preliminary work in head and neck cancer, based on our discovery of the presence of primitive cells within a variety of head and neck cancers and the involvement of the RAS in these tumours is an exciting avenue for further research.
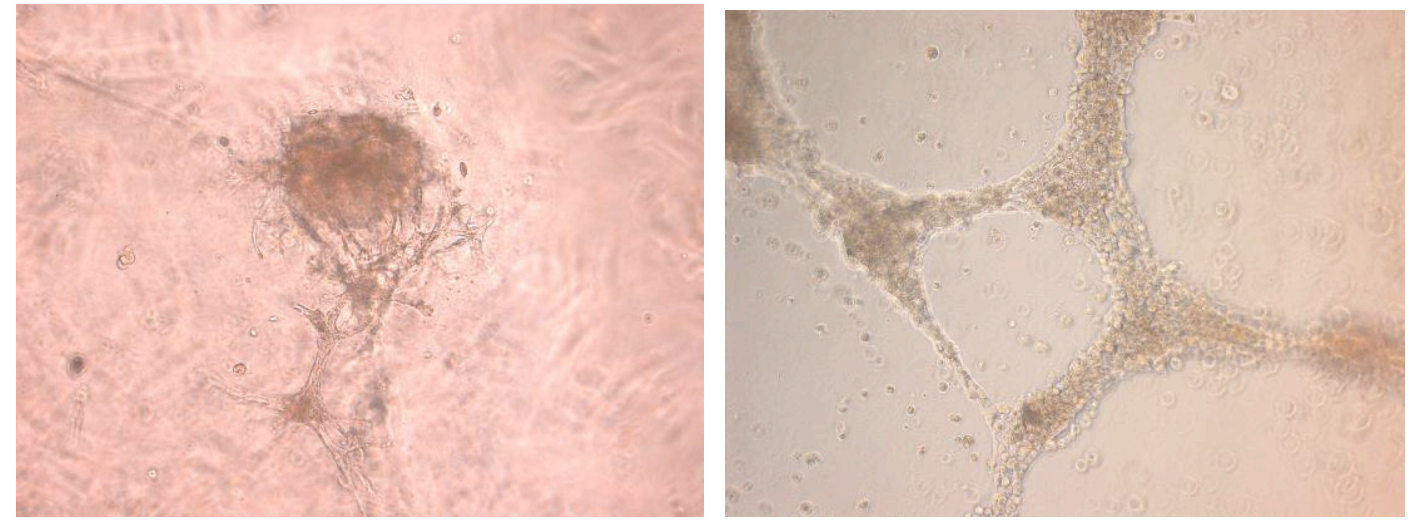

Figure 12.6. The formation of vascular tubes derived from endothelioma tumour cells requires the presence of IH stem cells when grown in matrigel. Images taken day 2 (left) and day 6 (right) in culture. 


\section{References}

1. Mulliken, JB. \& Glowacki, J. Hemangiomas and vascular malformations in infants and children: A classification based on endothelial characteristics. Plast Reconstr Surg 69, 412-419 (1982).

2. Takahashi, K., Mulliken, JB., Kozakewich, HPW., Rogers, RA., Folkman J. \& Ezekowitz. RAB. . Cellular markers that distinguish the phases of hemangioma during infancy and childhood. J Clin Invest 93, 2357-2364 (1994).

3. Frieden, IJ., Eichenfield, LF., Esterly, NB., Geronemus, R. \& Mallory, SB. Guidelines of care for hemangiomas of infancy. J Am Acad Dermatol 37, 631-637 (1997).

4. Tan, ST., Wallis, RA., He, Y. \& Davis, PF. Mast cells and hemangioma. Plast Reconstr Surg 113, 999-1011 (2004).

5. Tan, ST., Velickovic, M., Ruger, BM. \& Davis, PF. Cellular and extracellular markers of hemangioma. Plast \& Reconstr Surg 106, 529-537 (2000).

6. North, PE., Waner, M., Mizeracki, A. \& Mihm, MC. Glut1: A newly discovered immunohistochemical marker for juvenile hemangiomas. Hum Pathol 31, 11-22 (2000).

7. Mulliken, JB. Vascular birthmaks: hemangiomas and vascular malformations. Philidephia: WB Saunders (1988).

8. Itinteang, T., Tan, ST., Guthrie, S., Tan, CES., McIntyre, BC., Brasch, HD. \& Day, DJ. A placental chorionic villous mesenchymal core cellular origin for infantile haemangioma. J Clin Pathol. 2011 (accepted).

9. Picard, A., Boscolo, E., Khan, ZA., Bartch, TC., Mulliken, JB., Vazquez, MP. \& Bischoff, J. IGF-2 and FLT-1/VEGFR-R1 mRNA levels reveal distinctions and similarities between congenital and common infantile hemangioma. Pediatr Res. 63, 263-267 (2008). 
10. Horii, KA., Drolet, BA., Baselga, E., Frieden, IJ., Metry, DW., Morel, KD., Newell, BD., Nopper, AJ. \& Garzon, MC. Risk of Hepatic Hemangiomas in Infants With Large Hemangiomas. Arch Dermatol 146, 201-203 (2010).

11. Vishvanath, A. PhD Thesis: The Molecular Pathogenesis of Haemangioma. Victoria University of Wellington (2008).

12. Waner, M., North, PE., Scherer, KA., Frieden, IJ., Waner, A. \& Mihm, MC. The Nonrandom Distribution of Facial Hemangiomas. in Arch Dermatol 139, 869-875 (2003).

13. Metry, DW., Hawrot, A., Altman, C. \& Frieden, IJ. Association of Solitary, Segmental Hemangiomas of the Skin With Visceral Hemangiomatosis. Arch Dermatol 140, 591596 (2004).

14. Enjolras, O., Mulliken, JB., Boon, LM., Wassef, M., Kozakewich, HP. \& Burrows, PE. Noninvoluting congenital hemangioma: a rare cutaneous vascular anomaly. Plast Reconstr Surg 107, 1647-1654 (2001).

15. Berenguer, B., Mulliken, JB., Enjolras, O., Boon, LM., Wassef, M., Josset, P., Burrows, PE., Perez-Atayde, AR. \& Kozakewich, HP. Rapidly involuting congenital hemangioma: clinical and histopathologic features. Pediatr Dev Pathol. 6, 495- 510. (2003).

16. Finn, M., Glowacki, J. \& Mulliken, JB. Congenital vascular lesions: clinical application of a new classification. J Pediatr Surg 18, 894-900 (1983).

17. Batniji, RK., Buckingham, ED. \& Williams, EF. An aesthetic approach to facial hemangiomas. Arch Facial Plast Surg 7, 301-306 (2005).

18. Drolet, BA., Esterly, NB. \& Frieden, IJ. Hemangiomas in Children. N Eng/ J Med 341, 173-181 (1999).

19. Chang, LC., Haggstrom AN., Drolet, BA., Baselga, E., Chamlin, SL., Garzon, MC., Horii, KA., Lucky, AW., Mancini, AJ., Metry, DW., Nopper, AJ. \& Frieden, IJ. Growth 
characteristics of infantile hemangiomas: Implications for management. Pediatrics $122,360-367$ (2008).

20. Haggstrom, AN., Drolet, BA., Baselgia, E., Chamlin, SL., Garzon, MC., Horii, KA., Lucky, AW., Mancini, AJ., Metry, DW., Newell, B., Nopper, AJ. \& Frieden, IJ. Prospective Study of Infantile Hemangiomas: Clinical Characteristics Predicting Complications and Treatment. Pediatrics 118, 882-887 (2006).

21. Boon, LM., MacDonald, DM. \& Mulliken, JB. Complications of Systemic Corticosteroid Therapy for Problematic Hemangioma. Plast Reconstr Surg 104, 16161623 (1999).

22. Fawcett, SL., Grant, I., Hall, PN., Kelsall, AWR. \& Nicholson, JC. Vincristine as a treatment for a large haemangioma threatening vital functions. Brit J Plast Surg 57, 168-171 (2004).

23. Greinwald, JH., Jr, Burke, DK., Bonthius, DJ., Bauman, NM. \& Smith, RJH. An Update on the Treatment of Hemangiomas in Children With Interferon Alfa-2a. Arch Otolaryngol Head Neck Surg 125, 21-27 (1999).

24. Bigorre, M., Van Kien, AK. \& Valette, H. Beta-Blocking Agent for Treatment of Infantile Hemangioma. Plast Reconstr Surg 123, 195e-196e (2009).

25. Leaute-Labreze, C., Dumas de la Roque, E., Hubiche, T., Boralevi, F., Thambo, J. \& Taieb, A. Propranolol for Severe Hemangiomas of Infancy. in N Engl J Med 358, 2649-2651 (2008).

26. Tan, ST., Itinteang, T. \& Leadbitter, P. Low-dose propranolol for infantile haemangioma. J Plast Reconstr Aesth Surg 64, 292-299 (2011).

27. Manunza, F., Syed, S., Laguda, B., Linward, J., Kennedy, H., Gholam, K., Glover, M., Giradini, A. \& Harper, Jl. Propranolol for complicated infantile haemangiomas: a case series of 30 infants. Brit J Dermatol 162, 466-468 (2010). 
28. Smoller, BR. \& Alfelberg, DB. Infantile (juvenile) capillary hemangioma: A tumor of heterogeneous cellular elements. J Cutan Pathol. 20, 330-336 (1993).

29. Dosanjh, A., Chang, J., Bresnick, S., Zhou, L., Reinisch, J., Longaker, M. Karasek, M. In vitro characteristics of neonatal hemangioma endothelial cells: similarities and differences between normal neonatal and fetal endothelial cells. J Cutan Pathol 27, 441-450 (2000).

30. Boye, E., Yu, Y., Paranya, G., Mulliken, JB., Olsen, BR. \& Bischoff, J. Clonality and altered behavior of endothelial cells from hemangiomas. J Clin Invest 107, 745-752 (2001).

31. Ferreira, R., Ohneda, K., Yamamoto, M. \& Philipsen, S. GATA1 Function, a Paradigm for Transcription Factors in Hematopoiesis. in Mol Cell Biol 25, 1215-1227 (2005).

32. Ritter, MR., Reinisch, J., Friedlander, SF. \& Friedlander, M. Myeloid cells in infantile hemangioma. Am J Pathol 168, 621-628 (2006).

33. da Silva Meirelles, L., Caplan, AI. \& Nardi, NB. In Search of the In Vivo Identity of Mesenchymal Stem Cells. in Stem Cells 26, 2287-2299 (2008).

34. Jain, RK. Molecular regulation of vessel maturation. Nat Me 9, 685-693 (2003).

35. Peichev, M., Naiyer, AJ., Pereira, D., Zhu, Z., Lane, WJ., Williams, M., Oz, MC., Hicklin, DJ., Witte, L., Moore, MAS. \& Rafii, S. Expression of VEGFR-2 and AC133 by circulating human CD34+ cells identifies a population of functional endothelial precursors. Blood 95, 952-958 (2000).

36. Yu, Y., Flint, AF., Mulliken, JB., Wu, JK. \& Bischoff, J. Endothelial progenitor cells in infantile hemangioma. Blood 103, 1373-1375 (2004).

37. Kleinman, ME., Tepper, OM., Capla, JM., Bhatt, KA., Ceradini, DJ., Galiano, RD., Blei, F., Levine, JP. \& Gurtner GC. Increased circulating AC133, CD34 endothelial progenitor cells in children with hemangioma. Lymphat Res Biol. 4, 301-307 (2003). 
38. O'Donoghue, K. \& Fisk, NM. Fetal stem cells. Best Prac \& Res Clin Obst Gyn 18, 853875 (2004).

39. Yu, Y., Fuhr, J., Boye, E., Gyorffy, S., Soker, S., Atala, A., Mulliken, JB. Bischoff, J.. Mesenchymal stem cells and adipogenesis in hemangioma involution. Stem Cells 24, 1605-1612 (2006).

40. Nieto, M.A. The early steps of neural crest development. Mechanisms of Development 105, 27-35 (2001).

41. Chai, Y., Ito, Y. \& Han, J. TGF- $\beta$ Signaling and its Functional Significance in Regulating the Fate of Cranial Neural Crest Cells. Critical Reviews in Oral Biology \& Medicine 14, $78-88$ (2003).

42. Le Douarin, N.M., Creuzet, S., Couly, G. \& Dupin, E. Neural crest cell plasticity and its limits. Development 131, 4637-4650 (2004).

43. Cheung, M. \& Briscoe, J. Neural crest development is regulated by the transcription factor Sox9. in Development 130, 5681-5693 (2003).

44. Murakami, S., Kan, M., McKeehan, W. \& de Crombrugghe, B. Up-regulation of the chondrogenic sox9 gene by fibroblast growth factors is mediated by the mitogenactivated protein kinase pathway. PNAS 97, 1113-1118 (2000).

45. Lee, G., Kim, H., Elkabetz, Y., Al Sharmy, G., Pangiotakos, G., Barberi, T., Tabar, V. \& Studer, L. Isolation and directed differentiation of neural crest stem cells derived from human embryonic stem cells. Nat Biotech 25, 1468-1475 (2007).

46. Reid, P. \& Holen, I. Pathophysiological roles of osteoprotegerin (OPG). Euro J Cell Bio 88, 1-17 (2009).

47. Shipman, CM. \& Croucher, PI. Osteoprotegerin Is a Soluble Decoy Receptor for Tumor Necrosis Factor-related Apoptosis-inducing Ligand/Apo2 Ligand and Can 
Function as a Paracrine Survival Factor for Human Myeloma Cells. Can Res 63, 912916 (2003).

48. Ottersbach, K., Smith, A., Wood, A. \& Göttgens, B. Ontogeny of haematopoiesis: recent advances and open questions. Brit J Haem 148, 343-355 (2010).

49. Durand, C. \& Dzierzak, E. Embryonic beginnings of adult hematopoietic stem cells. haematologica 90, 100-108 (2005).

50. Tavian, M. \& Peault, B. Embryonic development of the human hematopoietic system. Int. J Dev. Biol. 49, 243-250 (2005).

51. Palis, J. \& Yoder, MC. Yolk-sac hematopoiesis: The first blood cells of mouse and man. Exp Hematol 29, 927-936 (2001).

52. Charbord, P., Tavian, M., Humeau, L. \& Peault, B. Early ontogeny of the human marrow from long bones: an immunohistochemical study of hematopoiesis and its microenvironment. Blood 87, 4109-4119 (1996).

53. Jaffredo, T., Gautier, R., Eichmann, A. \& Dieterlen-Lievre, F. Intraaortic hemopoietic cells are derived from endothelial cells during ontogeny. Development 125, 45754583 (1998).

54. North, T., et al. Cbfa2 is required for the formation of intra-aortic hematopoietic clusters. Development 126, 2563-2575 (1999).

55. Zovein, AC., Hofmann, JJ., Lynch, M., Frecnh, WJ., Turlo, KA., Yang, Y., Becker, MS., Zanetta, L., Dejana, E., Glasson, JC., Tallquist, MD. \& Iruela-Arispe, ML. Fate Tracing Reveals the Endothelial Origin of Hematopoietic Stem Cells. Cell Stem Cell 3, 625-636 (2008).

56. Wagner, R. Endothelial cell embryology and growth. Adv. Microcirc. 9, 45-75 (1980). 
57. Choi, K., Kennedy, M., Kazarov, A., Papadimitriou, JC. \& Keller, G. A common precursor for hematopoietic and endothelial cells. in Development 125, 725-732 (1998).

58. Nishikawa, SI., Nishikawa, S., Hirashima, M., Matsuyoshi, N. \& Kodama, H. Progressive lineage analysis by cell sorting and culture identifies FLK1+VE-cadherin+ cells at a diverging point of endothelial and hemopoietic lineages. Development 125, 1747-1757 (1998).

59. Fehling, HJ., Lacaud, G., Kubo, A., Kennedy, M., Robertson, S., Keller, G. \& Kouskoff, V. Tracking mesoderm induction and its specification to the hemangioblast during embryonic stem cell differentiation. in Development 130, 4217-4227 (2003).

60. Jaffredo, T., Bollerot, K., Sugiyama, D., Gautier, R. \& Drevon, C. Tracing the hemangioblast during embryogenesis: developmental relationships between endothelial and hematopoietic cells. Int. J. Dev. Biol. 49, 269-277 (2005).

61. Al-Mufti, R., Hambley, H., Farzaneh, F. \& Nicolaides, K. Fetal and embryonic hemoglobins in erythroblasts from fetal blood and fetal cells enriched from maternal blood in fetal anemia. haematologica 86, 1270-1276 (2001).

62. Peschle, C., Mavilio, F., Care, A., Migliaccio, G., Migliaccio, AR., Salvo, G., Samoggia, P., Petti, S., Guerriero, R., Marinucci, M., Lazzaro, D., Russo, G. \& Mastroberardino, G. Haemoglobin switching in human embryos: asynchrony of [zeta] [rarr] [alpha] and [epsi] [rarr] [gamma]-globin switches in primitive and definitive erythropoietic lineage. Nat 313, 235-238 (1985).

63. Schechter, AN. Hemoglobin research and the origins of molecular medicine. Blood 112, 3927-3938 (2008).

64. Bronner-Fraser, M. Neural crest cell formation and migration in the developing embryo. FASEB J 8, 699-706 (1994). 
65. Iwashita, T., Kruger, GM., Pardal, R., Kiel, MJ. \& Morrison, SJ. Hirschsprung Disease Is Linked to Defects in Neural Crest Stem Cell Function. in Science 301, 972-976 (2003).

66. Kim, J., Lo, L., Dormand, E. \& Anderson, DJ. SOX10 Maintains Multipotency and Inhibits Neuronal Differentiation of Neural Crest Stem Cells. Neuron 38, 17-31 (2003).

67. Krause, DS., Fackler, MJ., Civin, Cl. \& May, WS. CD34: structure, biology, and clinical utility in Blood 87, 1-13 (1996).

68. Yin, AH., Miraglia, S., Zanjani, ED., Almeida-Porada, G., Ogawa, M., Leary, AG., Olweus, J., Kearney, J. Buck, DW. AC133, a Novel Marker for Human Hematopoietic Stem and Progenitor Cells. in Blood 90, 5002-5012 (1997).

69. Huber, TL., Kouskoff, V., Joerg Fehling, H., Palis, J. \& Keller, G. Haemangioblast commitment is initiated in the primitive streak of the mouse embryo. Nat 432, 625630 (2004).

70. Tan, ST., Hasan, Q., Velickovic, M., Ruger, BM., Davis, RPF. \& Davis, PF. A novel in vitro human model of hemangioma. Mod Pathol 13, 92-99 (2000).

71. Richmond, TD., Chohan, M. \& Barber, DL. Turning cells red: signal transduction mediated by erythropoietin. Trends in Cell Biology 15, 146-155 (2005).

72. Sul, HS. Minireview: Pref-1: Role in Adipogenesis and Mesenchymal Cell Fate. Mol Endocrinol 23, 1717-1725 (2009).

73. Zambidis, ET., Park, TS., Yu, W., Tam, A., Levine, M., Yuan, X., Pryzhkova, M. \& Peault, B. Expression of angiotensin-converting enzyme (CD143) identifies and regulates primitive hemangioblasts derived from human pluripotent stem cells. Blood 112, 3601-3614 (2008).

74. Pipkin, FB., Smales, OR. \& O'Callaghan, MJ. Renin and angiotensin levels in children. Arch Dis Child 56, 298-302 (1981). 
75. Stephenson, TJ., Pipkin, FB. \& Elias-Jones, AC. Factors influencing plasma renin and renin substrate in premature infants. Arch Dis Child 66, 1150-1154 (1991).

76. Youmbissi, TJ., Tedong, F., Fairbank, ST., Blackett-Ngu, K. \& Mbede, J. Plasma Renin Activity Studies in a Group of African Neonates and Children. in J Trop Pediatr 36, 128-130 (1990).

77. Lavoie, JL. \& Sigmund, CD. Minireview: Overview of the Renin-Angiotensin System: An Endocrine and Paracrine System. Endocrin 144, 2179-2183 (2003).

78. Laslett, AL., Filipczyk, AA. \& Pera, MF. Characterization and Culture of Human Embryonic Stem Cells. Trends in Cardiovascular Medicine 13, 295-301 (2003).

79. Thomson, JA., Itskovitz-Eldor, J., Shapiro, SS., Waknitz, MA., Swiergiel, JJ., Marshall, VS. \& Jones, JM. Embryonic stem cells lines derived from human blastocysts. Science 282, $1145-1147$ (1998).

80. Xu, C., Inokuma, MS., Denham, J., Golds, K., Kunda, P., Gold, JD. \& Carpenter, MK. Feeder-free growth of undifferentiated human embryonic stem cells. Nat Biotech 19, 971-974 (2001).

81. Nichols, J., Zevnik, B., Anastassiadis, K., Niwa, H., Klewe-Nebenius, D., Chambers, I., Scholer, H. \& Smith, A. Formation of Pluripotent Stem Cells in the Mammalian Embryo Depends on the POU Transcription Factor Oct4. Cell 95, 379-391 (1998).

82. Burdon, T., Smith, A. \& Savatier, P. Signalling, cell cycle and pluripotency in embryonic stem cells. Trends in Cell Biology 12, 432-438 (2002).

83. Rodda, D., Chew, J., Lim, L., Loh, Y., Wang, B., Ng, H. \& Robson, P. Transcriptional Regulation of Nanog by OCT4 and SOX2. J Biol Chem 280, 24731-24737 (2005).

84. North, PE., Waner, M., Mizeracki, A., Mrak, RE., Nicholas, R., Kincannon, J., Suen, JY. \& Mihm, MC. A Unique Microvascular Phenotype Shared by Juvenile Hemangiomas and Human Placenta. Arch Dermatol 137, 559-570 (2001). 
85. Huang, SA., Tu, HM., Harney, JW., Venihaki, M., Butte, AJ. \& Kozakewich, HPW. . Severe Hypothyroidism Caused by Type 3 lodothyronine Deiodinase in Infantile Hemangiomas. N Engl J Med 343, 185-189 (2000).

86. Barnes, CM., Huang, S., Kaipainen, A., Sanoudou, D., Chen, EJ., Eichler, GS., Guo, Y., Ingber, DE., Mulliken, JB., Beggs, AH., Folkman, J. \& Fishman, SJ. Evidence by molecular profiling for a placental origin of infantile hemangioma. PNAS 102, 1909719102 (2005).

87. Bree, AF., Siegfried, E., Sotelo-Avila, C. \& Nahass, G. Infantile Hemangiomas: Speculation on Placental Trophoblastic Origin. Arch Dermatol 137, 573-577 (2001).

88. Kurman, RJ., Young, RH., Norris, HJ., Main, CS., Lawrence, WD. \& Scully, RE. Immunochemichemical localization of placental lactogen and chorionic gonadotrophin in the normal placenta and trophoblastic tumors, with emphasis on intermediate trophoblast and the placental site trophoblastic tumor. Int J Gynecol Pathol. 3, 101-121 (1984).

89. Blaschitz, A., Weiss, U., Dohr, G. \& Desoye, G. Antibody Reaction Patterns in First Trimester Placenta: Implications for Trophoblast Isolation and Purity Screening. Placenta 21, 733-741 (2000).

90. Itinteang, T., Brasch, H.D., Tan, S.T. \& Day, D.J. Expression of components of the renin-angiotensin system in proliferating infantile haemangioma may account for the propranolol-induced accelerated involution. J Plast Reconstr Aesth Surg 64, 759765 (2011).

91. Hempstock, J., Cindrova-Davies, T., Jauniaux, E. \& Burton, G. Endometrial glands as a source of nutrients, growth factors and cytokines during the first trimester of human pregnancy: a morphological and immunohistochemical study. Reprod Biol Endocrinol 2, 58 (2004). 
92. Ema, M., Yokomizo, T., Wakamatsu, A., Terunuma, T., Yamamoto, M. \& Takahashi, S. Primitive erythropoiesis from mesodermal precursors expressing VE-cadherin, PECAM-1, Tie2, endoglin, and CD34 in the mouse embryo. in Blood 108, 4018-4024 (2006).

93. Bauland, CG., Smit, JM., Bartelink, LR., Zondervan, HA. \& Spauwen, PHM. Hemangioma in the newborn: increased incidence after chorionic villus sampling. in Prenatal Diagnosis 30, 913-917 (2010).

94. Sans, V., de la Roque, ED., Berge, J., Grenier, N., Boralevi, F., Mazereeuw-Hautier, J., Lipsker, D., Dupuis, E., Ezzedine, K., Vergnes, P., Taieb, A. \& Leaute-Labreze, C. Propranolol for Severe Infantile Hemangiomas: Follow-Up Report. in Pediatrics 124, e423-431 (2009)

95. Denoyelle, F., Leboulanger, N., Enjolras, O., Harris, R., Roger, G. Garabedian, E. Role of Propranolol in the therapeutic strategy of infantile laryngotracheal hemangioma. Int J Ped Otor 73, 1168-1172 (2009).

96. Holmes, WJM., Mishra, A., Gorst, C. \& Liew, SH. Propranolol as first-line treatment for rapidly proliferating Infantile Haemangiomas. J Plast Reconstr Aesth Surg (doi:10.1016/j.bjps.2010.07.009).

97. Lisa, B., Umesh, D. \& Gresham, TR. Propranolol for airway hemangiomas: Case report of novel treatment. in Laryngoscope 9999 (2009).

98. Storch, $\mathrm{CH}$. \& Hoeger, PH. Propranolol for infantile haemangiomas: insights into the molecular mechanisms of action. Brit J Dermatol 163, 269-274 (2010).

99. Moran, CS., Cullen, B., Campbell, JH. \& Golledge, J. Interaction between Angiotensin II, Osteoprotegerin, and Peroxisome Proliferator-Activated Receptor- $\hat{I}^{3}$ in Abdominal Aortic Aneurysm. J Vasc Res 46, 209-217 (2009). 
100. Itinteang, T., Tan, ST., Brasch, H. \& Day, DJ. Haemogenic endothelium in infantile haemangioma. J Clin Pathol 63, 982-986 (2010).

101. Itinteang, T., Tan, ST., Brasch, HD., Vishvanath, A. \& Day, DJ. Primitive erythropoiesis in infantile haemangioma. Br J Dermatol 164, 1097-1100 (2011).

102. Itinteang, T., Tan, ST., Brasch, H. \& Day, DJ. Primitive mesodermal cells with a neural crest stem cell phenotype predominate proliferating infantile haemangioma. J Clin Pathol 63, 771-776 (2010).

103. Itinteang, T., Vishvanath, A., Day, D.J. \& Tan, S.T. Mesenchymal stem cells in infantile haemangioma. J Clin Pathol 64, 232-236 (2011).

104. Bajaj, A., Dyke P., Zaleski, C., Cava, J. \& McPherson E. Mild tessier no. 7 cleft with PHACE syndrome: the case for pulmonary vascular steal. Am J Med Gent Part A 155, 2298-2301 (2011).

105. Zaman, MA, Oparil, S \& Calhoun, DA. Drugs targeting the renin-angiotensinaldosterone system. Nat Rev Drug Discovery 1, 621-636 (2002).

106 Hendrix, MJC., Seftor, EA., Hess, AR. \& Seftor, REB. Vasculogenic mimicry and tumour-cell plasticity: lessons from melanoma. Nat Rev Can 3, 411-421 (2003) 